\title{
First International Conference on Nuclear Photonics: Abstract Book
}

, "First International Conference on Nuclear Photonics: Abstract Book," Proc. SPIE 10419, First International Conference on Nuclear Photonics, 1041901 (20 April 2017); doi: 10.1117/12.2280421

SPIE Event: First International Conference on Nuclear Photonics, 2016, Monterey, California, United States 

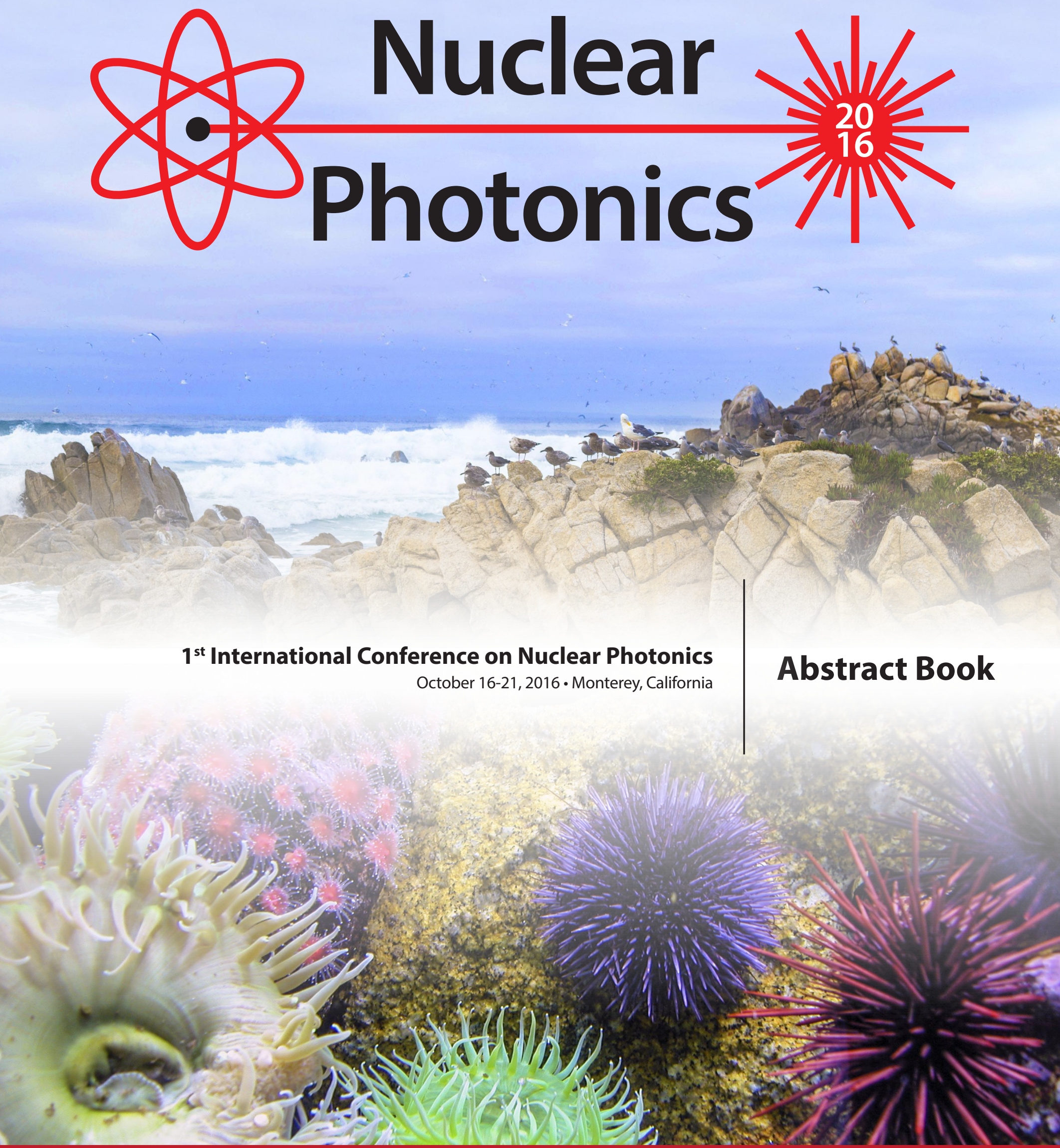

First International Conference on Nuclear Photonics, edited by Christopher Barty, Ryoichi Hajima, Norbert Pietralla Proc. of SPIE Vol. 10419, 1041901 · @ 2017 SPIE · CCC code: 0277-786X/17/\$18 · doi: 10.1117/12.2280421 


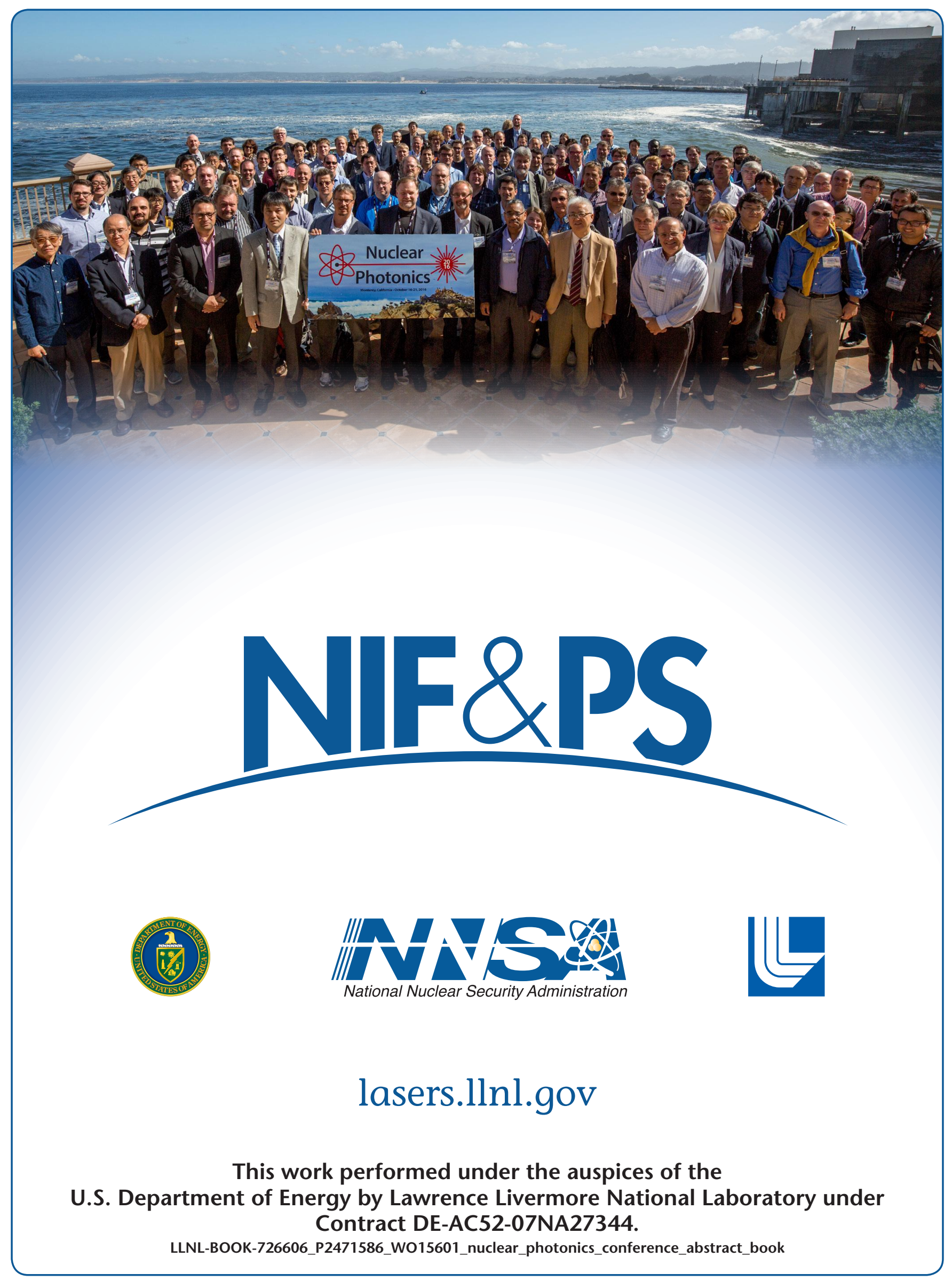

Proc. of SPIE Vol. 10419 1041901-2 


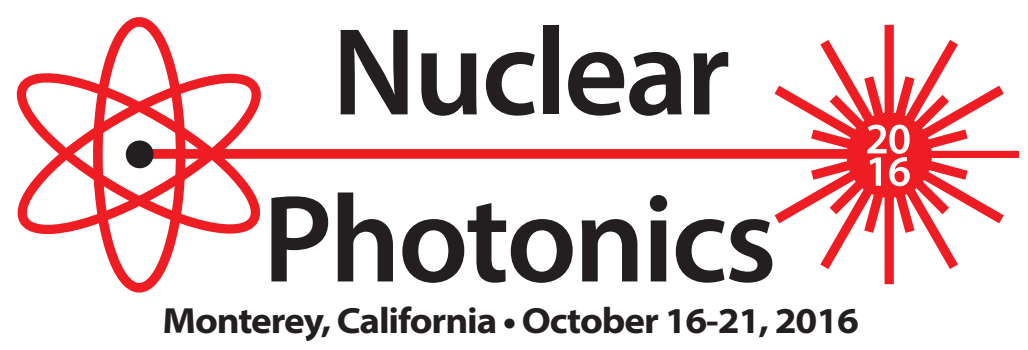

The pursuit of photon-based nuclear science and applications, i.e. Nuclear Photonics, is a rapidly evolving field of study that has been enabled by the development of ultra-bright, quasi-mono-energetic gamma-ray sources based on laser-Compton scattering and by the worldwide development of \$B-scale user facilities housing ultrahigh intensity lasers capable of producing field strengths of relevance to nuclear interactions.

Nuclear Photonics 2016 took place at the Monterey Plaza Hotel and Spa in Monterey, California from October 16th to the 21st, 2016. The conference brought together 144 participants from 17 countries and included experts in gamma-ray source development, ultrahigh intensity laser development, nuclear physics and nuclear-related applications.

Nuclear-related topics discussed during the meeting included;

- fundamental nuclear science and spectroscopy,

- nuclear medicine including radiography and radiotherapy,

- industrial non-destructive material imaging and evaluation,

- isotope-specific, nuclear materials detection and management,

- photo-fission and materials transmutation,

- photon-based production of rare isotopes,

- photon-enabled pulsed neutron generation and science,

- photon-enabled pulsed positron generation and science,

- photon-based hadron beams and applications,

- nuclear astrophysics and cosmology

- gamma-ray science above the giant dipole resonance

Sessions devoted to mono-energetic gamma-ray technology and to ultrahigh intensity laser technology were also a key part of the meeting. The former included discussion of the development of compact accelerators, optimization of laser-Compton interactions, novel detectors for bright gamma beams, gamma-ray monochromators, gamma-ray optics, advanced lasers for Compton light sources, high-brightness photoguns and novel scintillator materials. The latter included overviews of state-of-the-art laser facilities, advances in beam focusing and transport, novel pulse diagnostics, methods for control of pulse contrast, and the development of high average power, intense laser systems. Special efforts were made to integrate applications and technology development sessions so that each could motivate the other with respect to the development of nuclear photonics as a new scientific discipline. 


\section{Conference Chairs}

Dr. Christopher Barty, Lawrence Livermore National Laboratory, USA

Dr. Ryoichi Hajima, National Institutes for Quantum and Radiological Science and Technology, Japan

Prof. Norbert Pietralla, Technische Universität Darmstadt, Germany

\section{Program Chairs}

Prof. Calvin Howell, Triangle Universities Nuclear Laboratory, USA

Prof. Markus Roth, Technische Universität Darmstadt, Germany

\section{Program Committee}

Prof. Andrei Afanasev, George Washington University, USA

Dr. Michael Fazio, SLAC National Accelerator Laboratory, USA

Dr. Sydney Gales, Extreme Light Infrastructure Nuclear Physics, Romania

Dr. Cameron Geddes, Lawrence Berkeley National Laboratory, USA

Prof. Fazia Hannachi, University of Bordeaux, France

Dr. Kiminori Kondo, Institutes for Quantum and Radiological Science and Applications, Japan

Prof. Paul McKenna, University of Strathclyde, United Kingdom

Dr. Catalin Miron, Extreme Light Infrastructure Delivery Consortium, Europe

Prof. Shuji Miyamoto, University of Hyogo, Japan

Prof. Takashi Nakano, Osaka University, Japan

Prof. Hideaki Ohgaki, Kyoto University, Japan

Dr. Anton Tonchev, Lawrence Livermore National Laboratory, USA

Prof. Donald Umstadter, University of Nebraska, USA

Prof. Ying K. Wu, Duke University, USA

Prof. Andreas Zilges, University of Cologne, Germany

\section{Local Committee}

Trina Zagar

Mila Shapovalov

Brian Chavez

Jason Laurea

Pam Spears

Kim Casey

Connie Oakley

Melissa Olejniczak 

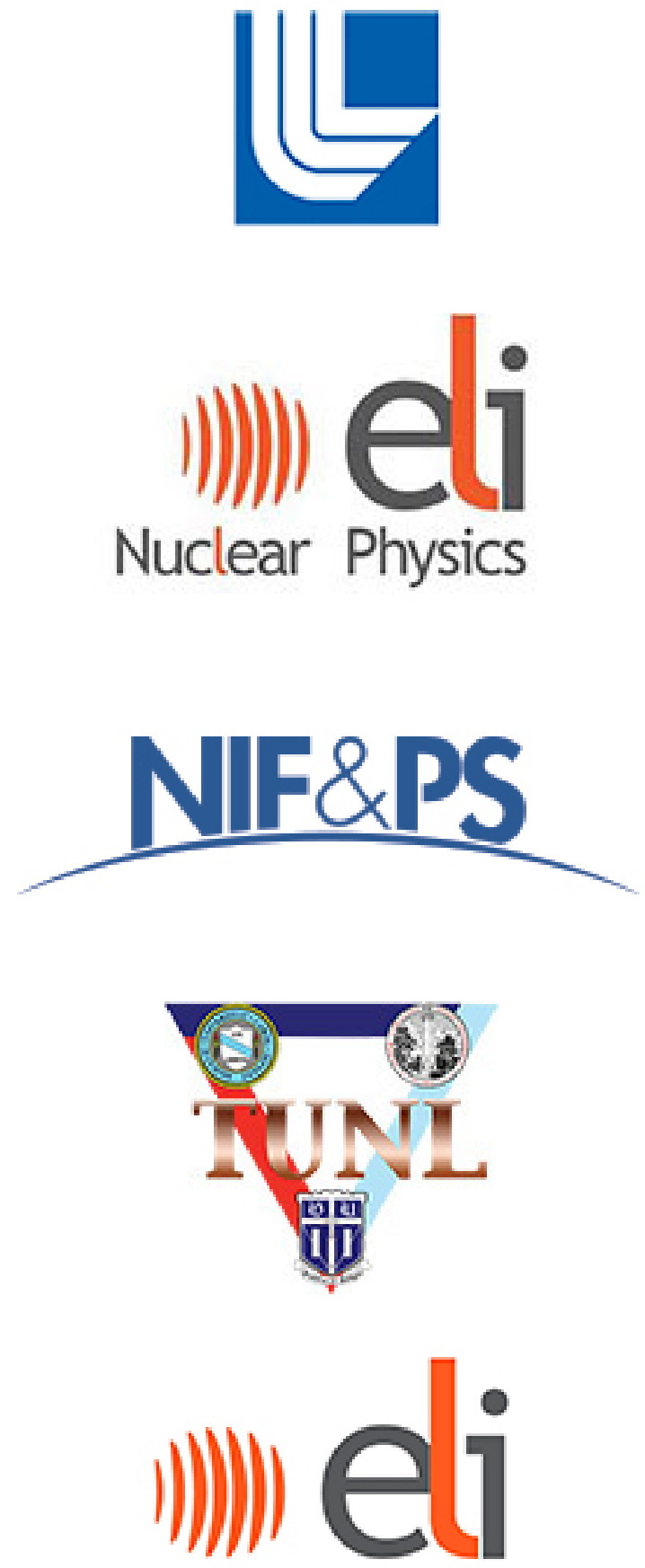

delivery consortium
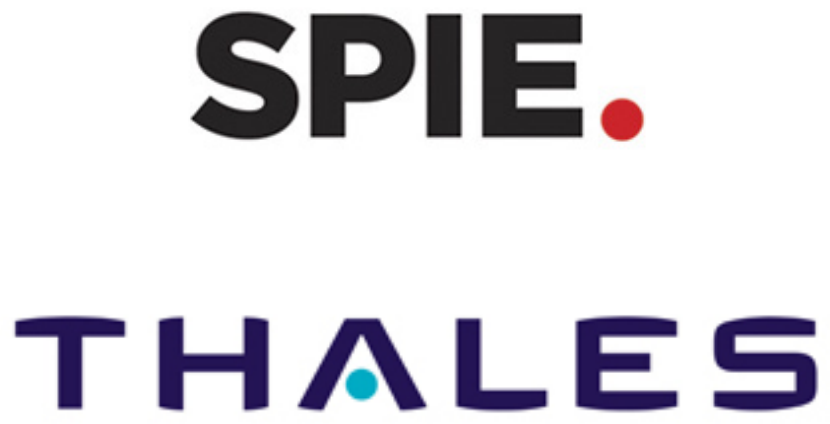

Proc. of SPIE Vol. $104191041901-5$

Downloaded From: https://www.spiedigitallibrary.org/conference-proceedings-of-spie on 26 Apr 2023 Terms of Use: https://www.spiedigitallibrary.org/terms-of-use 

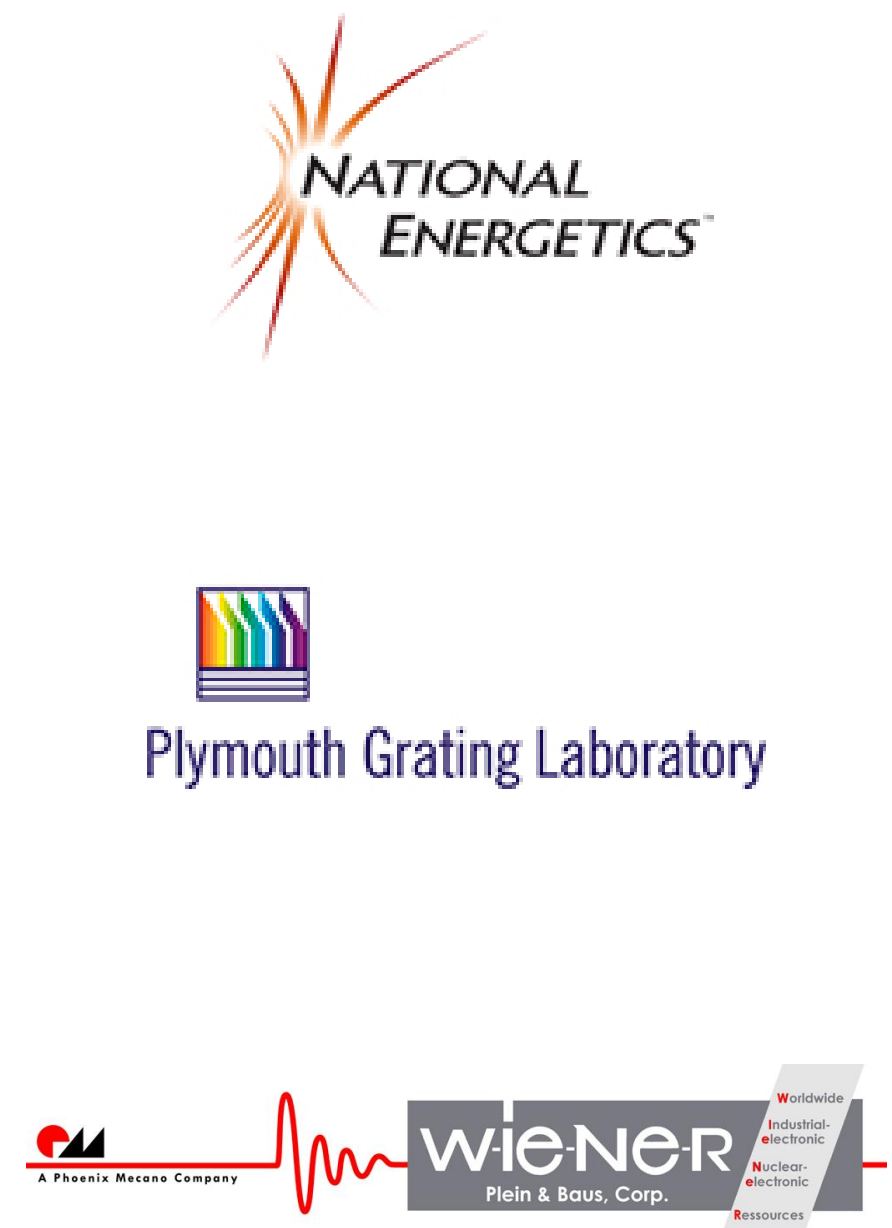

Proc. of SPIE Vol. 10419 1041901-6

Downloaded From: https://www.spiedigitallibrary.org/conference-proceedings-of-spie on 26 Apr 2023 Terms of Use: https://www.spiedigitallibrary.org/terms-of-use 
Conference Chairs $\ldots \ldots \ldots \ldots \ldots \ldots \ldots \ldots \ldots \ldots \ldots \ldots \ldots \ldots \ldots \ldots \ldots \ldots \ldots \ldots \ldots \ldots \ldots$

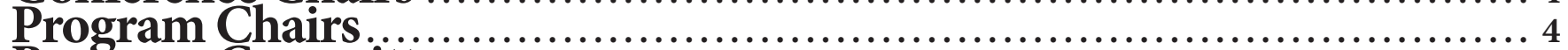

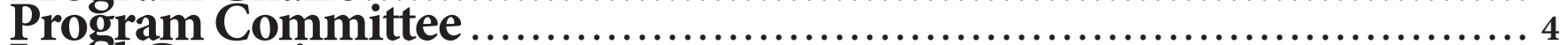

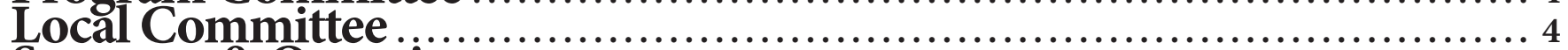

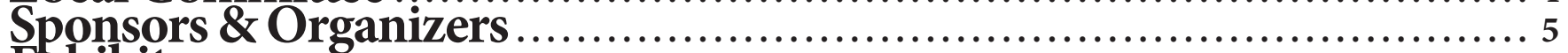

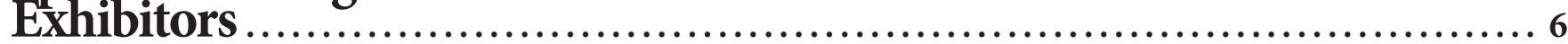

\section{Monday, October 17, 2016}

ELI-NP Gamma-Ray Facility and Research Program: 8:15 10:25

Session Chair: C. Barty

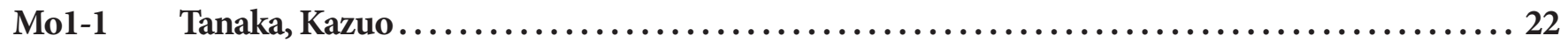

Extreme Light Infrastructure - Nuclear Physics - Romania

Overview and Status of the ELI-Nuclear Physics Project

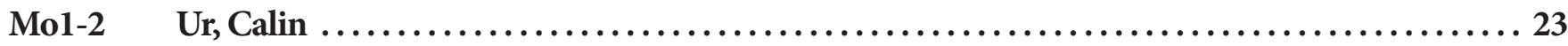

Extreme Light Infrastructure - Nuclear Physics - Romania

Science Perspectives of Nuclear Resonance Fluorescence Experiments at ELI-NP

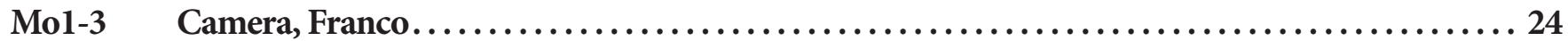

Istituto Nazionale di Fisica Nucleare - Italy

Science Perspectives on Gamma-above-neutron-threshold Experiments at ELI-NP

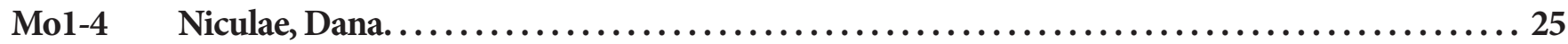

Horia Hulubei National Institute of Physics and Nuclear Engineering - Romania

Survey of Methods for Creation and Use of Medical Radio-isotopes

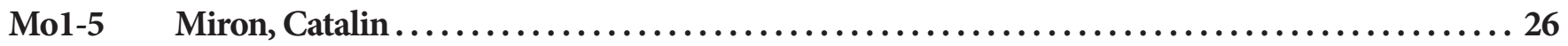

Extreme Light Infrastructure - Delivery Consortium - Europe

The Transition of ELI from Project to an International User Facility

\section{Ultrahigh Intensity Lasers \& Nuclear Physics: 10:45 12:40 Session Chair: M. Roth}

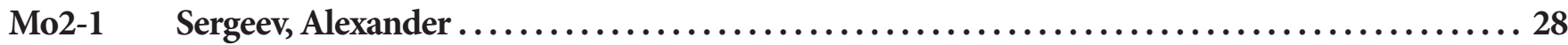

Institute for Applied Physics - Russia

High-brilliance gamma ray sources enabled by Exawatt-scale lasers

Mo2-2 Marklund, Mattias .................................................... 29

Chalmers University of Technology - Sweden

Ultra-bright $\mathrm{GeV}$ photon source via controlled electromagnetic cascades in laser-dipole waves

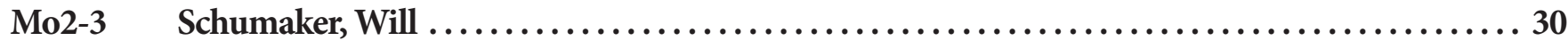

SLAC National Accelerator Laboratory - USA

Gamma-rays, Positrons and Pions from High Intensity Laser Interactions

Mo2-4 Gordon, Daniel .

Naval Research Laboratory - USA

Tunneling Ionization in Extreme Light 


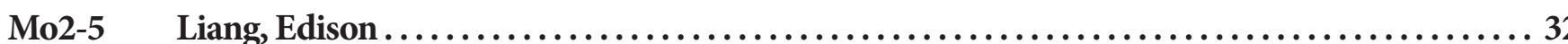

Rice University - USA

Dense Pair Creation and Gamma-ray Spectroscopy

\section{Accelerator-based Compton Sources: 14:00 16:05 Session Chair: C. Howell}

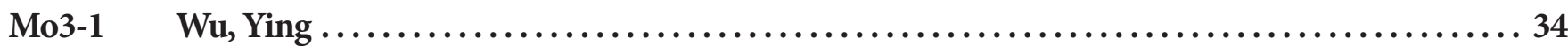

Duke University - USA

HIGS Overview - Capabilities and New Development

Mo3-2 Hajima, Ryoichi. ................................................. 35

National Institutes for Quantum and Radiological Science and Technology - Japan

ERL based laser-Compton sources and applications

Mo3-3 Litvinenko, Vladimir

Stony Brook University - USA

High Flux Compton Gamma-ray Sources above $100 \mathrm{MeV}$

Mo3-4 Cassou, Kevin...................................................... 38

Laboratoire de l'Accélérateur Linéaire - France

EuroGammaS Gamma Beam Source delivery for ELI-NP Overview and Status

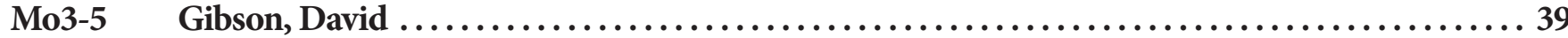

Lawrence Livermore National Laboratory - USA

High-flux, Ultra-narrow-bandwidth, Laser-Compton Light Source Architectures

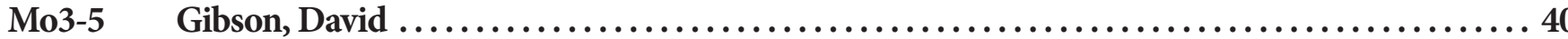

Lawrence Livermore National Laboratory - USA

High-flux, Ultra-narrow-bandwidth, Laser-Compton Light Source Architectures

\section{Photo-nuclear Physics: 16:25 18:20 Session Chair: M. Jentschel}

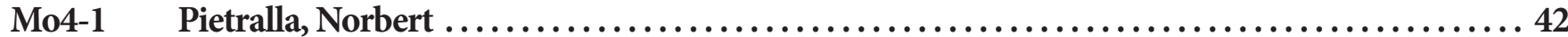
Technical Universität Darmstadt - Germany Photonuclear spectroscopy of discrete quantum states: basic principles, opportunities, and limitations

Mo4-2 Zilges, Andreas .................................................... 43

University of Cologne - Germany

Origin of Dipole Strength in Atomic Nuclei

Mo4-3 Gunst, Jonas ..................................................... 44

Max Planck Institute for Nuclear Physics, Heidelberg - Germany

Nuclear excitations on the $\mathrm{keV}$ and MeV energy scale

Mo4-4 Downie, Evangeline 45

George Washington University - USA

Exploring Polarizabilities with the MAMI A2 Tagged Photon Beam 
Future Gamma Systems Round Table

hosted by TUNL: 20:30 21:30

Tuesday, October 18, 2016

Extreme Lasers and Applications: 8:30 10:25

Session Chair: E. Liang

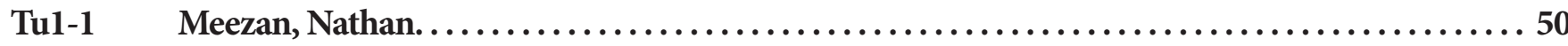

Lawrence Livermore National Laboratory - USA

Status of Laser Driven Nuclear Fusion at LLNL

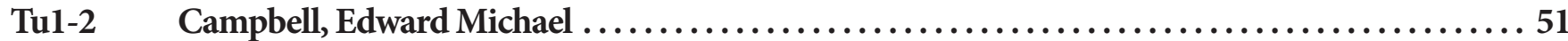

University of Rochester - USA

High-Peak-Power Laser Research at the Laboratory for Laser Energetics and

the Pathway to a 100-Petawatt Class Laser

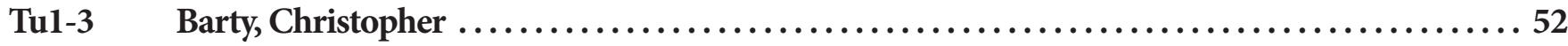

Lawrence Livermore National Laboratory - USA

Novel Exawatt Technologies and the path to laser intensities beyond $10 \mathrm{E} 24 \mathrm{~W} / \mathrm{cm} 2$

Tu1-4 Hannachi, Fazia.................................................. 53

University of Bordeaux - France

APOLLON: present status and perspectives

Tu1-5 Nishiuchi, Mamiko............................................. 54

Kansai Photon Science Institute - Japan

Novel Nuclear Applications of Laser-Driven Ion Acceleration

\section{Tuesday, October 18, 2016 \\ Nuclear Security Applications: 10:45 12:50 \\ Session Chair: R. Hajima}

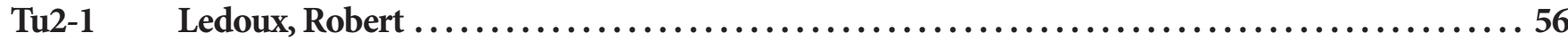

Passport Systems - USA

Non-intrusive Inspection Technologies for Nuclear Security and Nonproliferation

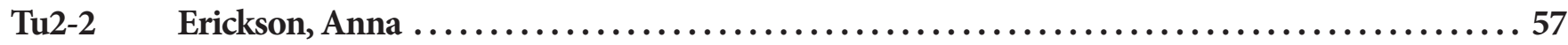

Georgia Institute of Technology - USA

Imaging in Active Interrogation: Comparison of Photon Beams

Tu2-3 Seya, Michio

National Institutes for Quantum and Radiological Science and Technology - Japan

Anticipations of NRF-based NDA of Nuclear Material Using Monochromatic Gamma-ray Beams

Tu2-4

Jovanovic, Igor

61

University of Michigan - USA

Active Interrogation by Nuclear Reaction-Based Sources and Particle Discriminating Detectors 
Tu2-5 Vogel, Sven .

Los Alamos National Laboratory - USA

Laser-driven Pulsed Neutron Sources as a Potential Pool-side Characterization Tool for

Nuclear Fuels

\section{High Energy Photon Systems \& Applications: 14:00 16:30 Session Chair: Y. Wu}

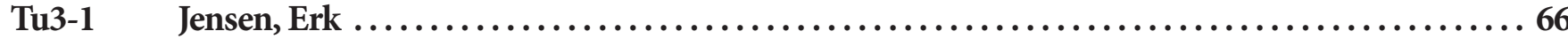

CERN - Switzerland

PERLE - a powerful ERL facility concept

Tu3-2 Miyamoto, Shuji ..................................................... 67

University of Hyogo - Japan

NewSUBARU Gamma Beam Source - Status and Activities

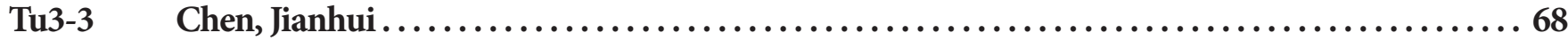

Shanghai Institute of Applied Physics (SINAP) - China

Gamma-ray Source Activities at Shanghai Synchrotron Radiation Facility

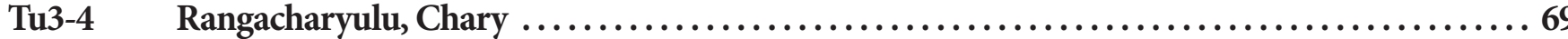

University of Saskatchewan - Canada

Photons for Food and Medicine

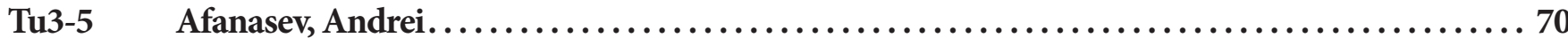

George Washington University - USA

Inverse-Compton Source of Twisted Photons

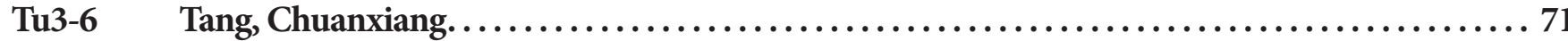

Tsinghua University - China

Mono-energetic $x /$ gamma ray sources based on ICS and their applications

\section{Advanced Optics \& Accelerator Technology: 16:50 18:45 Session Chair: I. Jovanovic}

Tu4-1 Tantawi, Sami........................................................ 74

SLAC National Accelerator Laboratory - USA

Future Perspectives on Ultra-Compact High Gradient RF Accelerator Technology

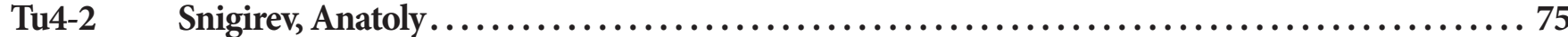

Immanuel Kant Baltic Federal University - Russia

20 years of $x$-ray Refractive Optics - Perspectives for MeV Light Sources

Tu4-3 Seiboth, Frank

DESY - Germany

Introduction to Refractive Hard X-ray Optics and Perspectives for Operation at MeV Energies 
Tu4-4

Guenther, Marc 78

Helmholtz Institute Jena - Germany

High precision refractive index measurements of several materials at $\gamma$-ray energies up to above $1 \mathrm{MeV}$ - Towards y refractive optics

Tu4-5 Jentschel, Michael 80

Institute Laue Langevin - France

Monochromatization of Gamma Rays to the eV level

\section{Evening Poster Session and Networking: 19:00 22:00 \\ Poster Abstracts can be found starting on page 147}

\section{Wednesday, October 19, 2016 \\ Nuclear Structure \& Astrophysics: 8:30 10:35 \\ Session Chair: N. Pietralla}

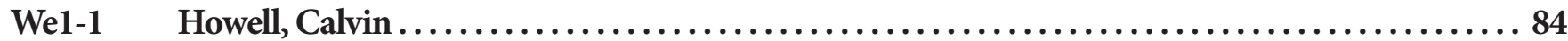

Triangle Universities Nuclear Laboratory - USA

Photonuclear Research at HI $\gamma$ S at Low Energies

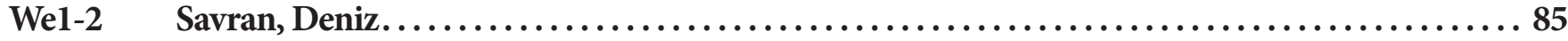

GSI Helmholtzzentrum für Schwerionenforschung - Germany

Decay properties of the Pygmy Dipole Resonance

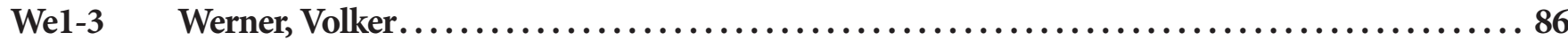

Technische Universität Darmstadt - Germany

Photonuclear Precision Measurements on Nuclear Quadrupole Collectivity

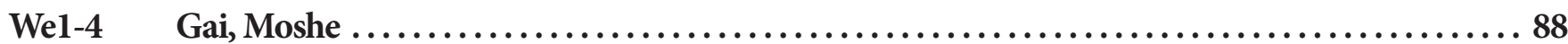

University of Connecticut - USA

Addressing Astrophysics Questions with laser-Compton Gamma-ray Sources

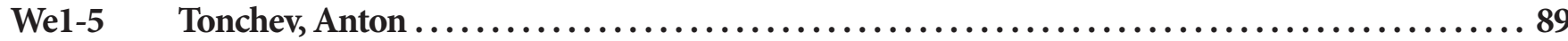

Lawrence Livermore National Laboratory - USA

s- Process Nucleosynthesis Using Nuclear Resonance Fluorescence

\section{Compton Sources Based on Intense Lasers: 10:55 13:15 Session Chair: C. Brenner}

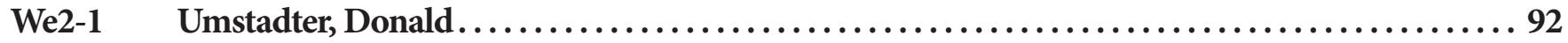

University of Nebraska - USA

Development and Applications of Laser-Wakefield-Accelerator-Driven Compton X-Rays

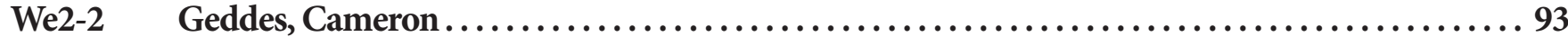

Lawrence Berkeley National Laboratory - USA

Compact quasi-monoenergetic photon sources for nuclear applications using

laser-plasma accelerators 
We2-3 Liu, Jiansheng..................................................... 94 SIOM - China

Bright $X$-and $\gamma$-ray sources based on a high-quality laser wakefield accelerator

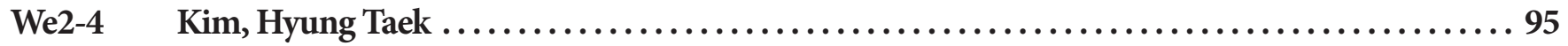

Guangju Institute of Science and Technology - Korea

Multi-GeV electron acceleration and PW Laser-driven Compton Gamma-rays

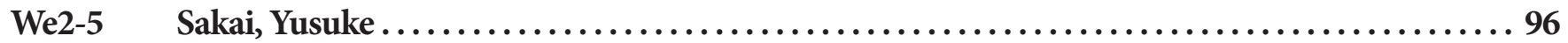

University of California at Los Angeles - USA

Physics and Technology of All-Optical Inverse Compton Scattering Sources

We2-6 Esarey, Eric .................................................... 97

Lawrence Berkeley National Laboratory - USA

Staging of laser-plasma accelerators to further accelerate or decelerate electron beams for

compact photon source applications

\section{Thursday, October 20, 2016 \\ Photon-based Particles Sources: 8:30 10:50 \\ Session Chair: K. Tanaka}

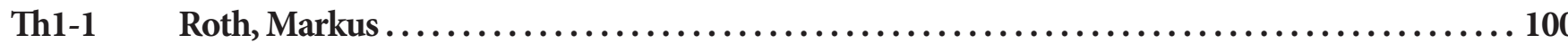

Technische Universität Darmstadt - Germany

Intense Ion, Neutron and X-Rays Beams from relativistic Laser-matter interaction

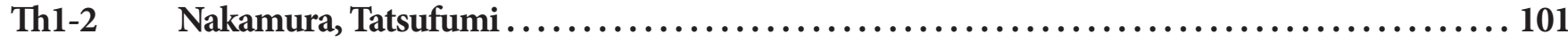

Fukuoka Institute of Technology - Japan

Laser-driven quantum beams from ultra-intense laser-matter interactions

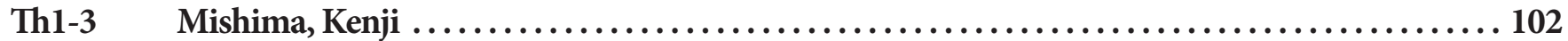

KEK - Japan

Pulsed Neutron Sources by Compton Gamma-ray Beams

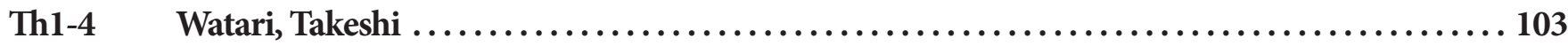

Hamamatsu - Japan

Demonstrations of neutron generation using solid-nanoparticle explosions driven by

DPSSL-pumped femtosecond laser pulse

Th1-5 Kondo, Kiminori.

Kansai Photon Science Institute - Japan

Laser Acceleration Studies in KPSI

Th1-6 Downer, Michael 106

University of Texas - USA

Production of 10 to $75 \mathrm{MeV}$ Compton Gamma-Rays from a $2 \mathrm{GeV}$

Laser-plasma electron accelerator 


\section{Nuclear Security \& Materials Management: 11:10 12:50 \\ Session Chair: A. Tonchev}

Th2-1 Plumeri, Stephane..................................................... 110

ANDRA - France

Non-destructive Testing for Control of Radioactive Waste - Overview of Challenges

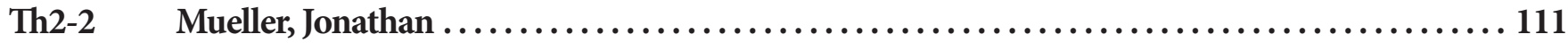

North Carolina State University - USA

Using Prompt Neutron Angular Distributions from Polarized Photofission to

Assay Special Nuclear Material

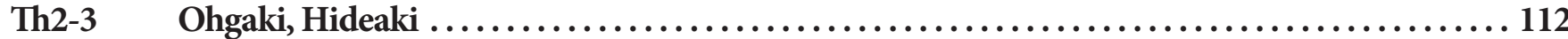

Kyoto University - Japan

NRF based Nondestructive Inspection System for SNM using Laser-Compton Gamma-rays

Th2-4 Ejiri, Hiro

Osaka University - Japan

Resonant Photonuclear Reactions for Neutrino Nuclear Responses and

Nuclear Isotope Detections

\section{Enabling Technologies for Nuclear Photonics: 14:15 16:00 Session Chair: F. Albert}

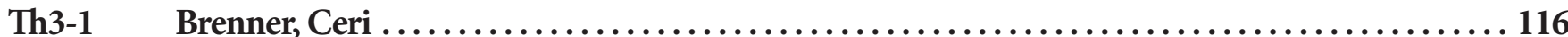

Rutherford Appleton Laboratory - United Kingdom

Laser-driven multi-modal beams for nuclear waste management inspection

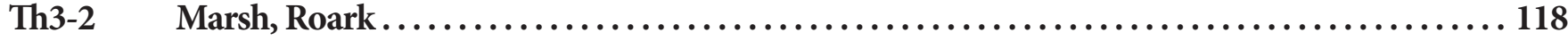

Lawrence Livermore National Laboratory - USA

Compact, High Brightness Accelerators for High-flux, Laser-Compton Sources

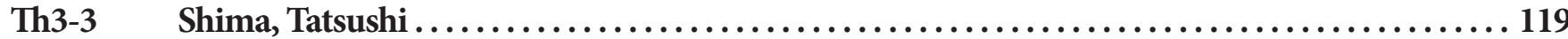

Osaka University - Japan

Ultra-low Background Counting Technique on the Earth's Surface

Th3-4 Martens, Aurelien ..................................................... 120

LAL - France

Gamma-beam monitoring with diamond sensors: fast, radiation hard and hardly destructive

Th3-5 Cwiok, Mikolaj

University of Warsaw - Poland

ELITPC - an Active-Target TPC for Studying Photonuclear Reactions with

High-Brilliance Gamma Beam at the ELI-NP Facility 


\section{Isotope-specific Detection, Assay and Imaging: 16:20 17:40 Session Chair: A. Afanasev}

Th4-1 Albert, Felicie.

Lawrence Livermore National Laboratory - USA

Experimental Demonstrations of Isotope-specific Material Detection and Imaging

Th4-2 Ludewigt, Bernhard .

Lawrence Berkeley National Laboratory - USA

Assessment of Near-monoenergetic Photon Sources for Nonproliferation Applications

Th4-3 Favalli, Andrea.

Los Alamos National Laboratory - USA

Laser-driven Neutrons for Active Interrogation of Special Nuclear Material

Th4-4 Oberstedt, Andreas

Extreme Light Infrastructure - Nuclear Physics - Romania

Determination of Photofission Fragment Characteristics of 234,238U and

232Th in the Barrier Region

\section{Friday, October 21, 2016 \\ Intense Laser Driven Sources and Technology: 8:30 10:30 Session Chair: D. Gibson}

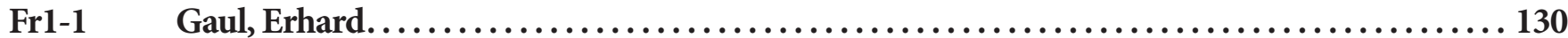

University of Texas - USA

Progress and Prospects far Lasers at 10PW and Beyond

Fr1-2 Wagner, Florian.

GSI - Germany

Improving laser-driven ion acceleration for applications in nuclear physics

Fr1-3 Lee, Kitae.

Korea Atomic Energy Research Institute - Korea

High-energy charged particles by laser-plasma interactions and applications

Fr1-4 Nees, John.

University of Michigan - USA

Detection and optimization methods for plasma interactions and nuclear processes

Fr1-5 Negoita, Florin.

Extreme Light Infrastructure - Nuclear Physics - Romania

Nuclear Isomer Gamma Spectroscopy as a Tool for

On-line Characterization of High Power Laser Generated Radiation

Fr1-6 Siders, Craig

Lawrence Livermore National Laboratory - USA

Application-enabling High Repetition PW Lasers: HAPLS Overview and Scaling 


\section{Gamma Source Technologies \& Applications: 10:50 13:00 Session Chair: J. Ness}

Fr2-1 Cherepy, Nerine...................................................... 138

Lawrence Livermore National Laboratory - USA

Advances in Scintillator Detector Materials

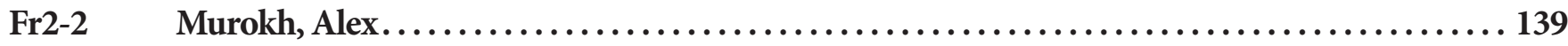

RadiaBeam - USA

Development of High Repetition Rate Inverse Compton Scattering Gamma Source with

Laser Pulse Recirculation

Fr2-3 Kosuge, Atsushi................................................ 140

KEK - Japan

Enhancement Cavity-based Laser-Compton Scattering Photon Source

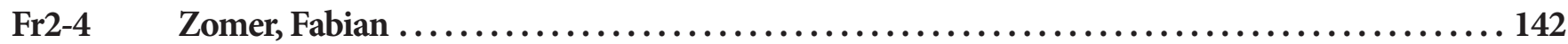

LAL - France

Developments of optical system for X/gamma ray Compton machines

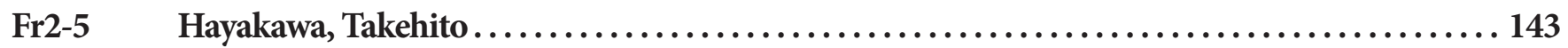

National Institutes for Quantum and Radiological Science and Technology - Japan

Neutron Angular Distribution in (gamma, $n$ ) Reactions with

Linearly Polarized Gamma-ray Beams

\section{Closing Remarks and Announcement Regarding Nuclear Photonics 2018}

\section{Tuesday, October 18, 2016 \\ Evening Poster Session and Networking: 19:00 22:00}

TuP-01

Hanayama, Ryohei . 148

The Graduate School for the Creation of New Photonics Industries - Japan

The Introduction of Development Project for

Laser Driven Compact Neutron Source in Hamamatsu

TuP-02 Marklund, Mattias

Chalmers University of Technology - Sweden

Aspects of quantum electrodynamics in laser-plasma and laser-particle interactions

TuP-03 Wu, Yuanbin.

Max Planck Institute for Nuclear Physics - Germany

Nuclear reactions of astrophysical interest in laser plasmas

TuP-04 Blackburn, Tom.

Chalmers University of Technology - Sweden

QED-driven laser absorption 
TuP-05

Bohlen, Simon

153

Deutsches Elektronen-Synchrotron - Germany

Simulations of Inverse Compton Scattering as Diagnostic for

Plasma Wakefield Electrons at FLASHForward

TuP-06 Covington, Aaron

University of Nevada-Renoo - USA

Production of Short-lived Positron Emitting Isotopes following the Rapid Acceleration of

Laser Ablation Plumes with a Pulsed-Power z-pinch Plasma Driver

TuP-07 Lozovoy, Vadim..................................................... 155

Michigan State University - USA

Designer spectral phases for stretching and compression of intense laser pulses

TuP-08 Nakai, Mitsuo.

Institute of Laser Engineering, Osaka University - Japan

A New Research Project on Laser-Driven Neutron sources and applications at ILE,

Osaka University

TuP-09 Park, Seong Hee.

Korea Atomic Energy Research Institute - South Korea

High-vacuum Laser-Driven Electron Accelerator for Compact Radiation Sources

TuP-10 Peatross, Justin....................................................... 158

Brigham Young University - USA

Ignatovsky Diffraction - Calculating Vector Fields in an Arbitrarily Tight Laser Focus since 1920

TuP-11

Qi, Rong.

ShangHai Institute of Optics and Fine Mechanics, Chinese Academy of Science - China

Diagnosis of Compton $\gamma$-ray sources based on a laser wakefield accelerator and

high- energy electron radiography

TuP-12 Qiao, Bin

Peking University - China

Brilliant Gamma-Ray Emission from Near-Critical Plasma Interaction with

Ultraintense Laser Pulses

TuP-13 Sang, Haibo

College of Nuclear Science and Technology, Beijing Normal University - China

Positron Acceleration In Plasma Bubble Wakefield Driven By An Ultraintense Laser

TuP-14 Serebryakov, Dmitry .............................................. 164

Institute of Applied Physics of the Russian Academy of Sciences - Russia

Collimated gamma-ray beam produced by laser-matter interaction in the grazing incidence regime

TuP-15 Shen, Lei

Shanghai Institute of Applied Physics, Chinese Academy of Sciences - China

Waveguide, Nonlinear Optics: From laser beam to electron measurement

TuP-16 Tsymbalov, Ivan. .

Institute for Nuclear Research RAS, Moscow, Russia - Russia

Gamma \& neutron production with femtosecond table top TW laser 
TuP-17 Wang, Wentao 167

Shanghai Institute of Optics and Fine Mechanics - China

Control of seeding phase for the cascaded laser wakefield accelerator with gradient injection

TuP-18

Xie, Baisong

Beijing Normal University - China

Electron-positron pair production from vacuum in

ultrastrong laser fields Baisong Xie ${ }^{\star}$ and Ziliang Li

TuP-19 Ruhl, Hartmut.

Ludwig-Maximilians-University - Germany

Variations on Mean Field Theory in Strong Optical Fields:

A New Simulation Model for Electron-Positron Plasma and Radiation

TuP-20 Choudhury, Deepika .

ELI-NP, IFIN-HH - Romania

High resolution study of transmission resonances for sub-barrier fission at ELI-NP

TuP-21 Constantin, Paul

Extreme Light Infrastructure - Nuclear Physics - Romania

Photofission Experiments at the ELI-NP Facility

TuP-22 Gai, Moshe

University of Connecticut - USA

The C/O Ratio in Stellar Helium Burning: Measurements of the $16 O(\gamma, \alpha)$

12C Reaction With an Optical TPC at HI $\gamma S^{*}$

TuP-23 Gambacurta, Danilo

Extreme Light Infrastructure - Nuclear Physics - Romania

Multipole response in atomic nuclei within the Second Random Phase Approximation

TuP-23 Gambacurta, Danilo

Extreme Light Infrastructure - Nuclear Physics - Romania

Multipole response in atomic nuclei within the Second Random Phase Approximation

TuP-24 Gayer, Udo .

Institut fuer Kernphysik, Technische Universitaet Darmstadt - Germany

Studying low-lying electric dipole strength in atomic nuclei with

quasi-monoenergetic gamma-rays

TuP-25 Ghita, Dan Gabriel

IFIN-HH - Romania

Monte Carlo simulations for ELI-GANT detection array at ELI-NP

TuP-26 Guardo, Giovanni Luca....

Laboratori Nazionali del Sud - Italy

Development of the ELISSA array: first tests and simulations

TuP-26 Guardo, Giovanni Luca

Laboratori Nazionali del Sud - Italy

Development of the ELISSA array: first tests and simulations 
TuP-27 Kong, Xiangjin. 180

Max Planck Institute for Nuclear Physics - Germany

Using nuclear transitions to control and store $x$-ray photons

TuP-28 Matei, Catalin.

Extreme Light Infrastructure - Nuclear Physics - Romania

Overview of the Gamma Beam Monitoring Instruments at ELI-NP

TuP-29 Nedorezov, Vladimir .

Institute for Nuclear Research RAS - Russia

Near threshold photonuclear reactions

TuP-30 Praharaj, C Rajendra .

Institute of Physics, Bhubaneswar, India - India

K Isomers in neutron-rich Rare-earth nuclei*

TuP-31 Snigireva, Irina

European Synchrotron Radiation Facility - France

High energy coherent X-ray microscopy for mesoscopic materials

TuP-32 Dupraz, Kevin ...

CNRS / Laboratoire de l'Accélérateur Linéaire - France

The Thom X X-ray source

TuP-33 Murphy, Chris ...

University of York - United Kingdom

Exploring Stong-Field QED with Ultra-Intense Lasers

TuP-34 Hwang, Yoonwoo

University of California, Irvine - USA

Characterization of Laser-Compton Sources at LLNL

TuP-35 Juergensen, Lars Erik.

Technische Universität Darmstadt - Germany

Enhancement of the Superconducting Darmstadt S-DALINAC

Accelerator to an Energy-Recovery LINAC

TuP-36 Katoh, Masahiro

Institute for Molecular Science, National Institutes of Natural Sciences - Japan

Twisted Gamma-rays from Nonlinear Inverse Compton Scattering of Circular Polarized Light

TuP-37 Muto, Toshiya.

Research center for electron photon science, Tohoku university - Japan

the characterization of GeV gamma-ray beam via Bremsstrahlung in the storage ring in ELPH, To

TuP-38 Ndiaye, Cheikh Fall

Laboratoire de l'Accélérateur Linéaire - France

Status of the Development of ELI-NP's Laser Beam Circulator

TuP-39 Petrillo, Vittoria.

University of Milan - Italy

Compton sources for the observation of elastic photon-photon scattering events 
TuP-40 Petrillo, Vittoria....................................................... 196

University of Milan - Italy

Compton scattered $x$-gamma rays with orbital momentum

TuP-41 Rykovanov, Sergey ............................................... 197

Helmholtz Institute Jena - Germany

Ponderomotive Broadening in Nonlinear Compton Scattering

TuP-42 Tomkus, Vidmantas................................................... 198

Center for Physical Sciences and Technology - Lithuania

Plasma Guiding and Scattering Microstructures for Laser Driven Thompson Sources

TuP-43 Vaccarezza, Cristina ................................................... 199

Istituto Nazionale di Fisica Nucleare- Laboratori Nazionali di Frascati - Italy

Beam dynamics studies for the RF Linac of the ELI-NP Gamma Beam System.

TuP-44 Ponomarev, Vladimir .............................................. 200

Technical University of Darmstadt - Germany

Pygmy dipole resonance in atomic nuclei

Index.

201

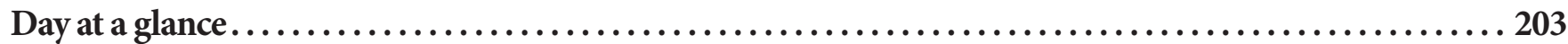

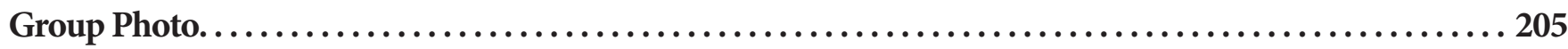




\section{Page Intentionally Left Blank}

Proc. of SPIE Vol. 10419 1041901-20 Downloaded From: https://www.spiedigitallibrary.org/conference-proceedings-of-spie on 26 Apr 2023
Terms of Use: https://www.spiedigitallibrary.org/terms-of-use 


\section{Monday, October 17, 2016 \\ ELI-NP Gamma-Ray Facility and Research Program: 8:15 10:25 \\ Session Chair: C. Barty}




\title{
Over-view and strategy of the ELI-Nuclear Physics Project
}

\author{
Kazuo A. Tanaka and Nicolae-Victor Zamfir \\ Extreme Light Infrastructure-Nuclear Physics (ELI-NP) \\ Bucharest-Magurele, Romania
}

Since chirped pulse amplification scheme[1] has changed the game in high energy density physics, the available laser intensity has kept increasing, can reach $10^{23} \mathrm{~W} / \mathrm{cm}^{2}$ or even higher, and can deliver radiation higher than the previously used in nuclear facilities. In order to make use of this capability in full depth, a laser-centered, distributed pan-European research infrastructure, involving ultra-intense laser technologies with ultra-short pulses was triggered through the European Light Infrastructure (ELI) project at the state of the art and beyond.

The European Forum of Infrastructure (ESFRI) has selected in 2006 a proposal of constructing a 200J laser system with intensities up to $10^{22}-10^{23} \mathrm{~W} / \mathrm{cm}^{2}$, called ELI at the site of BucharestMagurele, Romania. The rest of two large scale high intensity ELI laser facilities are built in The Czech Republic, and Hungary[2]. The scientific research at ELI-NP includes two areas where only little experimental results were reported until now. The first one is laser-driven experiments related to nuclear physics, strong-field quantum electrodynamics and associated vacuum effects. The second area is that of experiments based on a Compton-backscattering high-brilliance and intense low-energy $(<20 \mathrm{MeV})$ gamma beam, a combination of laser and accelerator technology at the frontier of knowledge.

Typical experiments planned in the early stage[3] will be introduced after the system overview.

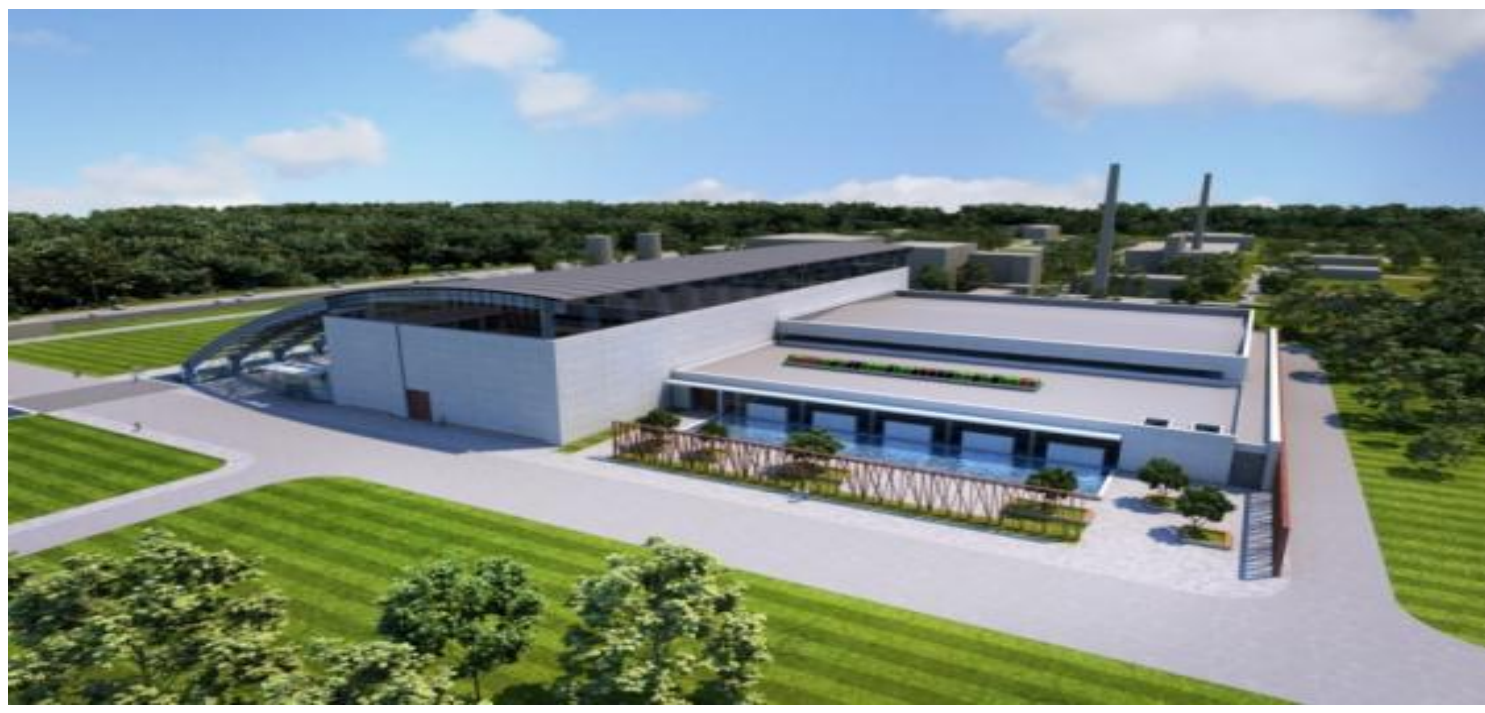

\section{Reference}

Figure 1. Artist view of ELI-NP facility

1. D Strickland and G Mourou, Opt. Commun. 56, 219 (1985).

2. https://eli-laser.eu/

3. Romanian Reports in Physics, 68, Supplement, pp. S3-S443 (2016). 


\title{
Science Perspectives of Nuclear Resonance Fluorescence Experiments at ELI-NP
}

\author{
C.A. $\mathrm{Ur}^{1}$ \\ ${ }^{1}$ ELI-NP, IFIN-HH, Magurele, Bucharest, Romania \\ calin.ur@eli-np.ro
}

Energy tunable quasi-monochromatic $\gamma$-ray systems based on Inverse Compton Scattering (ICS) of intense laser photon pulses off highly-relativistic electron beams, such as the Gamma Beam System of Extreme Light Infrastructure - Nuclear Physics (ELI-NP) [1,2], open new perspectives for NRF studies of nuclear structure. Inverse Compton Scattering processes provide currently the most brilliant sources of photons in the $\gamma$-ray energy regime for which the parameters like energy, bandwidth, time structure or polarization are controllable by experimentalists. Nuclear structure investigation via NRF measurements represents one of the main research activities in the photonuclear program of ELI-NP. The combination of intense monochromatic photon beams with high-resolution $\gamma$-ray spectroscopy of the following decays offers the best conditions to study in detail the decay behavior of photo-excited states [3].

The Technical Design Report for the NRF experimental setup [4] was completed based on the physics cases of interest. The proposed physics cases are imagined to take full advantage of the outstanding features of the gamma beams delivered at ELI-NP, such as high spectral density of $\sim 10^{4}$ photons $/ \mathrm{s} \cdot \mathrm{eV}$, small bandwidth of $\lesssim 0.5 \%$, linear polarization, small geometrical size of the $\gamma$ beam. The physics cases aim at studying the electromagnetic dipole response of rare and radioactive nuclei or to identify exotic weak structures in the nuclei.

The main experimental setup for NRF measurements at the Gamma Beam System of ELINP [4] will consist of a compact array of gamma-ray detectors placed around the target. Stateof-the-art detectors will be used in the array with the aim to: a) maximize the solid angle covered with detectors placed in close geometry around the target; b) optimize the response of the array to $\gamma$ rays over a wide range of energies from few hundred $\mathrm{keV}$ to several $\mathrm{MeV}$; $\mathrm{c}$ ) allow for precise $\gamma$-ray polarization and angular distribution measurements. The array for NRF nuclear structure studies was named ELIADE (ELI-NP Array of DEtectors) and will consist of large volume HPGe segmented CLOVER detectors and large volume $\mathrm{LaBr}_{3}(\mathrm{Ce})$ scintillator detectors.

A summary of the NRF physics cases proposed for being studied at ELI-NP and the associated experimental setup will be discussed in the presentation.

1. N.V. Zamfir et al., "Extreme Light Infrastructure - Nuclear Physics (ELI-NP) European Research Center”, Eur. Phys. J. Web of Conference 66, 11043 (2014).

2. O. Adriani et al., "Technical Design Report; EuroGammaS proposal for the ELI-NP Gamma beam System", arXiv:1407.3669v1 [physics.acc-ph] (2014).

3. B. Loher et al., "The high-efficiency $\gamma$-ray spectroscopy setup $\gamma^{3}$ at HI $\gamma \mathrm{S}$ ", Nucl. Instr. Meth. Phys. Res. A723, 136 (2013).

4. C.A. Ur et al., "Nuclear Resonance Fluorescence at ELI-NP", Rom. Rep. Physics 68, S483 (2016). 


\title{
Science Perspectives on Gamma-above-neutron-threshold Experiments at ELI-NP
}

\author{
F.Camera $^{1}$, H. Utsunomiya ${ }^{2}$, D. Filipescu ${ }^{3}$ \\ 1-Universita di Milano and INFN section of Milano \\ 2-Department of Physics, Konan University, Kobe, Japan \\ 3-ELI-NP, Magurele, Bucharest, Romania
}

\begin{abstract}
Photon-induced nuclear reactions with energies higher than the particle binding energy mainly excite collective states like the Isovector Giant Dipole Resonance (IVGDR or simply GDR). The photon and neutron decay of GDR to the ground state and excited states provides information on the decay strength of GDR with (in case of photon decay) multipole selectivity and the coupling of GDR to low-frequency collective modes. In case of neutron decay, discrepancies have been revealed in measured partial $(\gamma, \mathrm{xn})$ cross sections, therefore there is a growing interest of constructing a new compilation of total and partial photoneutron cross sections.

The Pygmy Dipole Resonance (PDR) was discovered at the turn of the $21^{\text {st }}$ century in the lowenergy tail of GDR. The PDR which shows up in the energy region $6-12 \mathrm{MeV}$ may represent a new collective excitation mode of a dipole oscillation of neutron-skin against a core nucleus. Understanding the PDR is important to clarify the entire E1 response of nuclei. Besides, the PDR may be a good probe of the nuclear symmetry energy in the equation of state (EOS) for nuclear matter, which is of direct relevance to the formation of neutron stars in the type II supernova explosion.

Extremely intense and monochromatic gamma-ray beams offered by the ELI-NP will open up a new era of experimental photonuclear reaction study. The Physics cases and the experimental setup addressed by the ELI-NP Working Group "Gamma Above Neutron Threshold" (ELIGANT) will be discussed.

Two different detection systems have been designed, one (ELIGANT-TN) for Thermal Neutron measurements and the other (ELIGANT-GN) for Gamma and fast Neutron measurements (coincidence measurements are also foreseen). The ELIGANT-GN will consists of $\mathrm{LaBr}_{3}: \mathrm{Ce}+$ $\mathrm{CeBr}_{3}$ scintillation detectors for gamma rays and BC501A liquid and GS20 Li glass scintillators for the detection of neutrons.
\end{abstract}




\title{
Survey of Methods \\ for Creation and Use of Medical Radioisotopes
}

\author{
Dana Niculae ${ }^{1}$, Doina Draganescu ${ }^{1}$, Dimiter Balabanski ${ }^{2}$, Calin A. $\mathrm{Ur}^{2}$ \\ ${ }^{1}$ Horia Hulubei National Institute for Physics and Nuclear Engineering, IFIN-HH, Magurele, \\ Ilfov,Romania,dana.niculae@nipne.ro,doina.draganescu@nipne.ro \\ ${ }^{2}$ Extreme Light Infrastructure-Nuclear Physics, IFIN-HH, Magurele, Ilfov, Romania, \\ dimiter.balabanski@eli-np.ro,calin.ur@eli-np.ro
}

Radioisotopes applications in nuclear medicine are in the field of both diagnosis (oncology, cardiology and neurology) and therapy (oncology). Molecular imaging probes, a special class of radiopharmaceuticals, targets specific biochemical signatures associated with disease and allow for non-invasive imaging on the molecular level. Because changes in biochemistry occur before diseases reach the advanced stage, molecular imaging probes make it possible to locate and stage disease, track the effectiveness of drug, treat disease, monitor response, and select patients to allow for more personalized diagnosis and treatment of disease. Based on the same biochemical processes, radionuclide systemic therapy is a powerful method to eradicate disseminated tumor cells and small metastases.

Thus, to improve the differential diagnosis, prognosis, planning and monitoring of cancer treatment, new functional radiopharmaceuticals based on relevant bioactive molecules and promising medical radioisotopes have to be developed and evaluated. The potential interest of a given radio-isotope in medicine depends on a number of factors: the specific decay properties of the radio-isotope to be used; physical and biological half-life (which must be long enough to reach the target but short enough to avoid unnecessary radiation exposure); elemental/chemical properties (purification, post-processing and radiolabelling of bioactive molecules); pharmaceutical formulation constrains; and the ease of production (specific activity, cost effectiveness, availability).

As one of the alternative route for production of emerging/promising radioisotopes for nuclear medicine, ELI-NP will employ $(\mathrm{g}, \mathrm{n})$ nuclear reaction to produce such radioisotopes, with relevant quantity and quality. Prospective radioisotopes to be produced by (g,n) reactions simulations of the target geometry and estimation of activity of some radioisotopes of interest for nuclear medicine will be presented. 


\title{
The Transition of ELI from Project to an International User Facility
}

\author{
Catalin Miron ${ }^{1}$ \\ ${ }^{1}$ Extreme Light Infrastructure Delivery Consortium (ELI-DC), rue Montoyer 23, Brussels, \\ Belgium, c/o ELI Nuclear Physics / IFIN-HH, Bucharest-Măgurele, Romania \\ Catalin.Miron@eli-laser.eu
}

The Extreme Light Infrastructure ELI [1] will be the world's first international user facility for laser-based research and applications.

ELI is dedicated to fundamental research in the radiation-matter interaction field in the laser intensity regime up to $10^{23} \mathrm{~W} / \mathrm{cm}^{2}$, and beyond. Its laser technologies are mainly based on chirped pulse amplification of femtosecond optical pulses in broadband solid-state laser materials and/or nonlinear crystals. Single-beam laser peak-power will exceed 10 PW (10 ${ }^{16}$ Watt), while several beams will be possible to simultaneously use for experiments. Diode pumping will allow for up to $10 \mathrm{~Hz}$ operation at the multi-Petawatt level, while a bright, tunable gamma-ray beam will also be available for nuclear physics studies.

ELI will offer novel user research opportunities, from photonuclear science to tracking ultrafast electro-nuclear wave packets in atoms and molecules, or applications of secondary radiation and particle beams in materials science and for biomedical applications.

After its selection by the European Strategy Forum on Research Infrastructures (ESFRI) to become part of the European roadmap of Research Infrastructures, ELI is presently being implemented as a distributed research facility in the Czech Republic, Hungary and Romania. The buildings are finalized and the installation of equipment started already. While 2017 will be mainly dedicated to the commissioning of the lasers and secondary sources, user operation is scheduled to commence in 2018, with a progressive ramp-up of the capabilities and available beamtime for the international users. ELI will be operated as a European Research Infrastructure Consortium (ELI-ERIC) to be created by the European Commission in 2017.

1. https://eli-laser.eu. 


\section{Monday, October 17, 2016 \\ Ultrahigh Intensity Lasers \& Nuclear Physics: 10:45 12:40 \\ Session Chair: M. Roth}




\title{
High-brilliance gamma ray sources enabled by Exawatt-scale lasers
}

\author{
A.M.Sergeev ${ }^{1}$, A.V.Bashinov ${ }^{1}$, A.A.Gonoskov ${ }^{1,2}$, E.S. Efimenko ${ }^{1}$, A.A.Muraviev ${ }^{1}$, A.V.Kim ${ }^{1}$ \\ 1) Institute of Applied Physics of Russian Academy of Sciences, 46 Ul'yanov Street, 603950, \\ Nizhny Novgorod, Russia, \\ tel. (831)4365736, fax (831)4363792, \\ 23) Department of Applied Physics, Chalmers University of Technology, Gothenburg, Sweden \\ e-mail:ams@ufp.appl.sci-nnov.ru
}

Creation of the XCELS laser with 0.2 Exawatt peak power as well as other facilities of this class will open up opportunities for studying and exploiting new gamma ray sources currently unavailable in laboratory. The main advantage of these facilities is the capability to operate in the intensity range $\mathrm{I}>\mathrm{I}_{\mathrm{RD}} \sim 10^{24} \mathrm{~W} / \mathrm{cm}^{2}$. Overcoming this threshold dramatically changes the ultrarelativistic dynamics of electrons in the laser field. They begin to oscillate in the radiation dominated regime and efficiently convert energy from the optical to gamma range. In turn, flows of hard gamma quanta formed in the radiation-dominant mode provoke, in the area of strong optical fields, generation of electron-positron pairs and the chain reaction of developing electromagnetic cascades. The arising avalanche is a way to form an overdense electron-positron plasma coexisting with an overdense gas of gamma photons with energies $100 \mathrm{MeV}-1 \mathrm{GeV}$ and beyond.

In this report we will present a description of new physical prospects and experiments that can be performed at the XCELS facility that is under design at IAP RAS. In particular, we will demonstrate how to create electron-positron plasma with particle concentration amounting to $10^{27} \mathrm{~cm}^{-3}$ coexisting with gamma photons with concentration $>10^{26} \mathrm{~cm}^{-3}$. These exotic gamma ray sources can be made well controllable and directed with the brilliance achieving $610^{29}$ $\mathrm{s}^{-1} \mathrm{mrad}^{-2} \mathrm{~mm}^{-2}$. The scientific case for such upcoming Exawatt-scale lasers is highly compelling and includes new physics of nuclear excitation, gamma-gamma collisions, and probing the timespace structure of quantum vacuum. 


\title{
Ultra-bright GeV photon source via controlled electromagnetic cascades in laser-dipole waves
}

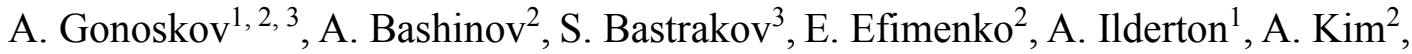 \\ M. Marklund ${ }^{1}$, I. Meyerov ${ }^{3}$, A. Muraviev², A. Sergeev ${ }^{2}$ \\ ${ }^{1}$ Department of Physics, Chalmers University of Technology, SE-41296 Gothenburg, Sweden \\ arkady.gonoskov@chalmers.se, anton.ilderton@chalmers.se,mattias.marklund@chalmers.se \\ ${ }^{2}$ Institute of Applied Physics, Russian Academy of Sciences, Nizhny Novgorod 603950, Russia \\ abvk@inbox.ru,evgeny.efimenko@gmail.com,kim@ufp.appl.sci-nnov.ru, \\ sashamur@gmail.com,ams@ufp.appl.sci-nnov.ru \\ ${ }^{3}$ Lobachevsky State University of Nizhni Novgorod, Nizhny Novgorod 603950, Russia \\ sergey.bastrakov@gmail.com,meerov@vmk.unn.ru
}

The large-scale laser facilities of the next generation, such as XCELS [1], will be capable of triggering the cascaded production of electron-positron pairs and high-energy photons. Apart from the fundamental interest, this process can serve for creation a new kind of gamma-ray source with extraordinary high flux. Such source might open up new possibilities for fundamental studies of electromagnetic and nuclear processes.

However, in strong laser fields a particle frequently emits photons that carry away a substantial part of the particle's energy. This might naturally prevent particles from reaching high energies and thus from emitting high-energy photons. Furthermore, a substantial part of generated highenergy photons might decay into electron-positron pairs before leaving the region of strong laser fields. Finally, the creation of electron-positron plasma by the cascade can quickly lead to the reduction of the field strength and thus can reduce energies of particles and photons. In many previously considered configurations of the laser fields these processes naturally restrict the efficient generation of photons to sub-GeV energies.

In our study, we propose and systematically analyze a concept of $\mathrm{GeV}$ photon source based on triggering a controlled cascade in a laser-formed dipole wave. In this configuration the anomalous radiative trapping (ART) [2] triggers an optimal regime of particle dynamics, governs the cascade and provides a directed generation of photons with $\mathrm{GeV}$ energies. We use our comprehensively extended 3D particle-in-cell code [3] to simulate and analyze the process and to find the optimal conditions. We also assess realistic conditions for creation of the dipole wave with 12 laser pulses as proposed in [2]. We demonstrate that the concept is already feasible for the facilities of total peak power at the level of $10 \mathrm{PW}$. A higher peak power of $40 \mathrm{PW}$ can provide $10^{9}$ photons with $\mathrm{GeV}$ energies in a well-collimated beam of just 4.5 fs. This source would be a powerful tool for studying fundamental electromagnetic and nuclear processes.

1. XCELS: www.xcels.iapras.ru.

2. A. Gonoskov et al., "Anomalous radiative trapping in laser fields of extreme intensity" Phys. Rev. Lett., 113, 014801 (2014).

3. A. Gonoskov et al., "Extended particle-in-cell schemes for physics in ultrastrong laser fields: Review and developments" Phys. Rev. E, 92, 023305 (2015). 


\title{
Gamma-rays, Positrons, and Pions from High-Intensity Laser Interactions
}

\author{
Will Schumaker ${ }^{1,2}$, Taiee Liang ${ }^{1}$, Siegfried Glenzer ${ }^{1}$, \\ Michael Vargas ${ }^{2}$, Karl Krushelnick ${ }^{2}$, Alec Thomas ${ }^{2}$, \\ Gianluca Sarri ${ }^{3}$, Daraugh Corgan ${ }^{3}$, Matt Zepf $^{3}$, \\ Kristjan Poder ${ }^{4}$, Jason Cole ${ }^{4}$, Stewart Mangles ${ }^{4}$, Zulfikar Najmudin ${ }^{4}$ \\ ${ }^{1}$ SLAC National Accelerator Laboratory, Menlo Park, CA, USA \\ wschumak@stanford.edu \\ ${ }^{2}$ University of Michigan, Ann Arbor, MI, USA \\ ${ }^{3}$ Queen's University of Belfast, Belfast, United Kingdom \\ ${ }^{4}$ Imperial College London, London, United Kingdom
}

Experimental results of laser wakefield acceleration (LWFA) of $\sim \mathrm{GeV}$ energy electrons driven by the 200TW HERCULES and the 400TW ASTRA-GEMINI laser systems and their subsequent generation of photons, positrons, and neutrons are presented. In LWFA, highintensity $\left(\mathrm{I}>10^{19} \mathrm{~W} / \mathrm{cm}^{2}\right)$, ultra-short $(\mathrm{t}<50 \mathrm{fs}$ ) laser pulses drive highly nonlinear plasma waves which can trap nano-Coloumbs of electrons and accelerate them to $\sim \mathrm{GeV}$ energies over $\sim$ cm lengths. These electron beams can then be converted by a high- $Z$ target via bremsstrahlung into low-divergence $(<20 \mathrm{mrad})$ beams of high-energy $(>500 \mathrm{MeV})$ photons and subsequently into positrons via the Bethe-Heitler process[1]. By increasing the material thickness and $\mathrm{Z}$, the

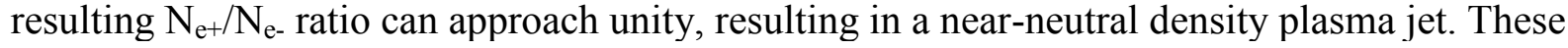
quasi-neutral beams are presumed to retain the short-pulse $(\mathrm{t}<40 \mathrm{fs})$ characteristic of the electron beam, resulting in a high peak flux/density of $\mathrm{n}_{\mathrm{e}-/ \mathrm{e}+} \sim 10^{16} \mathrm{~cm}^{-3}$, making the source an excellent candidate for laboratory study of astrophysical leptonic jets[2].

Alternatively, the electron beam can be interacted with a counter-propagating, ultra-high intensity $\left(\$ \mathrm{I}>10^{19} \mathrm{~W} / \mathrm{cm}^{2}\right.$ ) laser pulse to undergo inverse Compton scattering and emit a highpeak brightness beam of high-energy photons. These high-energy photon beams can be spectrally resolved using a forward Compton scattering spectrometer[3]. Moreover, nuclear activation in a secondary converter target generates $(\gamma, n)$ neutrons and even $(\gamma, \pi)$ pions from the higher-energy photons $(>150 \mathrm{MeV})$ which can be subsequently measured via activation analysis.

This research was supported by DOE/NSF-PHY 0810979, NSF CAREER 1054164, DARPA AXiS N66001-11-1-4208, SF/DNDO F021166, and the Leverhulme Trust ECF-2011-383.

1. G. Sarri, W. Schumaker, et al., "Table-top, laser-based source of femtosecond, collimated, ultra-relativistic positron beams," Physical Review Letters, 110, 255002 (2013).

2. G. Sarri, et al., "Generation of neutral and high-density electron-positron pair plasmas in the laboratory," Nature Communications, 6, 6467 (2015).

3. W. Schumaker, et al. "Measurements of high-energy radiation generation from laserwakefield accelerated electron beams," Physics of Plasmas, 21, 6467 (2014). 


\title{
Tunneling Ionization in Extreme Light
}

\author{
Daniel F. Gordon, Bahman Hafizi, and John Palastro \\ Naval Research Laboratory, Plasma Physics Division, Washington, DC, USA \\ daniel.gordon@nrl.navy.mil
}

Moderately heavy atoms (Z 20) are expected to be fully stripped via tunneling ionization when illuminated by a paraxially focused, multi-petawatt laser pulse. The two K-shell electrons, being much more tightly bound than the others, are abruptly exposed to an extreme field upon ionization, and are accelerated to super-ponderomotive energies through a wave-particle resonance mechanism. The photoelectron distribution has remarkable features, including high energy and directionality, complex correlations in momentum space, and discrete peaks that can be mapped to a particular crest of the optical wave. The process leaves behind bare nuclei, which are slightly accelerated by the laser fields. The effect of radiation reaction becomes interesting in the multi-exawatt regime. 


\title{
Dense Pair Creation and Gamma-Ray Spectroscopy
}

\author{
Edison Liang ${ }^{1}$ \\ ${ }^{1}$ Rice University, Houston, TX, USA \\ liang@rice.edu
}

On behalf of the Rice-UT collaboration, I will summarize recent results of ultra-intense laser experiments to create dense pair plasmas and gamma-ray sources using the Texas Petawatt Laser (TPW) to irradiate solid high-Z targets. With laser intensities exceeding $10^{\wedge} 21 \mathrm{~W} \cdot \mathrm{cm}^{\wedge} 2$ irradiating large thick $\mathrm{Pt}$ and $\mathrm{Au}$ targets, we demonstrated the feasibility of creating pairdominated plasmas and gamma-rays at record high emergent densities. A new type of gammaray spectrometer, called the scintillator attenuation spectrometer (SAS), was successfully demonstrated. We will discuss the design and results of the SAS. Potential applications of all these results will also be presented. 


\section{Monday, October 17, 2016 Accelerator-based Compton Sources: 14:00 16:05 Session Chair: C. Howell}




\title{
HIGS Overview - Capabilities and New Development
}

\author{
Ying K. $\mathrm{Wu}^{1}$ \\ ${ }^{1}$ HIGS/TUNL, and Department of Physics, Duke University, Durham, NC 27708-0319, USA \\ wu@fel.duke.edu
}

Since late 1970s, laser driven Compton gamma-ray beam facilities have been developed, constructed and operated around the world for basic science research in nuclear physics and astrophysics, and for applied research in the areas of national security and industrial applications. Currently, TUNL's High Intensity Gamma-ray Source (HIGS) located at Duke University campus is the most intense Compton gamma-ray beam facility dedicated for scientific research. Driven by a high power storage ring Free-Electron Laser (FEL), HIGS produces nearly monochromatic, highly polarized gamma-ray beams from 1 to $100 \mathrm{MeV}$, with its peak performance of total flux up to about $3 \mathrm{E} 10 \mathrm{~g} / \mathrm{s}$ and a spectral flux of more than $1 \mathrm{E} 3 \mathrm{~g} / \mathrm{s} / \mathrm{eV}$ around $10 \mathrm{MeV}$ region. In this talk, we will describe the present gamma-ray capabilities of the HIGS, as well as new capabilities enabled by a FEL wiggler switchyard system. We will also outline the future development and upgrade projects at the HIGS facility on the energy front and intensity front.

This work is supported in part by U.S. DoE grant, DE-FG02-97ER41033. 


\title{
ERL-based Laser Compton Sources and Applications
}

\author{
R. Hajima ${ }^{1,2}$ \\ ${ }^{1}$ National Institutes for Quantum and Radiological Science and Technology, \\ Shirakata 2-4, Tokai, Naka, Ibaraki 319-1106, Japan \\ 2 Japan Atomic Energy Agency Science and Technology \\ Shirakata 2-4, Tokai, Naka, Ibaraki 319-1195, Japan \\ hajima.ryoichi@qst.go.jp
}

Laser Compton scattering is the only practical method to produce energy-tunable and quasi-monoenergetic photon beams in $\mathrm{MeV}$ energies. We are proposing Compton sources based on energy recovery linacs (ERL). ERLs have been developed mainly for high-power free electron lasers and future synchrotron light sources but has advantages in Compton sources as well, because the electron beam of high-average current and small emittance in ERLs contribute directly to generation of high-flux and narrow-bandwidth $\mathrm{MeV}$ photons via Compton scattering[1]. An energy-recovery linac combined with a laser enhancement cavity is an ideal apparatus to realize a high-flux and narrow-bandwidth Compton source. An ERL-based Compton source to produce $2-\mathrm{MeV}$ photons with a flux of $10^{13} \mathrm{ph} / \mathrm{s}$ was designed for a nuclear industrial application, non-destructive assay of nuclear materials ${ }^{[2]}$.

In order to develop technologies necessary for such future ERL-based Compton sources, a research program was established in Japan. In this program, generation of Compton scattered photon beam from an energy-recovery linac was demonstrated at the Compact ERL (cERL). The Compact ERL is a facility of energy-recovery linac constructed as a test bed for future ERL-based photon sources such as synchrotron $\mathrm{X}$-ray, high-power FELs and Compton sources [3]. In the experiment, X-ray photon energy was measured to be $6.95 \mathrm{keV}$ and bandwidth for the detector half-opening angle, $0.14 \mathrm{mrad}$, was $0.4 \%$ (rms). The count rate of X-ray photons at the detector was $1370 \mathrm{cps}$, which corresponds to the source flux of $2.6 \times 10^{7} \mathrm{ph} / \mathrm{s}$. Detail description of the experiment is seen elsewhere ${ }^{[4]}$. The successful demonstration of Compton scattered $\mathrm{X}$-ray generation at the cERL clearly shows that critical technologies for a high-flux and narrow-bandwidth Compton source have been established. The technologies cover a wide range: generation and transportation of a small emittance beam, stable operation of an energy-recovery linac, a laser cavity for enhancing laser pulse energy by several orders of magnitude, precise synchronization and spatial overlapping of electron and laser beams for collision. Another experiment with a $10^{-} \mathrm{mA}$ electron beam and a 
$100-\mathrm{kW}$ laser beam is planned at the cERL to produce Compton scattered photons with a flux over $10^{11} \mathrm{ph} / \mathrm{s}$ in near future.

Utilizing this high-flux and narrow-bandwidth gamma-ray beam in combination with nuclear resonant fluorescence (NRF), we can make nondestructive measurement of arbitrary nuclides ${ }^{[5]}$. Such nondestructive measurement can be applied to nuclear security and safeguards. We have studied nondestructive measurement of isotopes in scattering and transmission geometries. A demonstration experiment with the transmission geometry was carried out for measuring a proxy target (Al) surrounded by absorbing material simulating a realistic spent fuel storage canister ${ }^{[6]}$. In the experiment, we confirmed that the canister material should not significantly influence the non-destructive assay. For the scattering geometry, we proposed to measure NRF transitions to the first excited state to avoid the coherent scattering contribution, which is a background for the NRF signals ${ }^{[7]}$. In both transmission and scattering geometries, generation of a high-flux and narrow-bandwidth $\mathrm{MeV}$ photons from the ERL-based Compton source is a key technology.

In this talk, we present the current development status and future perspectives of ERL-based Compton sources and overview possible applications utilizing such Compton source.

This work was supported by a Government (MEXT) Subsidy for Strengthening Nuclear Security and by Photon and Quantum Basic Research Coordinated Development Program from MEXT.

[1] R. Hajima, Rev. Acc. Sci. Tech. 3, 121 (2010).

[2] R. Hajima et al., Nucl. Instr. Meth. A 608, S57 (2009).

[3] S. Sakanaka et al., Proc. 2015 Particle Accelerator Conference, p.1359 (2015).

[4] T. Akagi et al., submitted to Phys. Rev. Accel. Beams.

[5] R. Hajima, et al., J. Nucl. Sci. Tech. 45, 441 (2008).

[6] C.T. Angell et al., Nucl. Instr. Meth. B 348, 11-19 (2015).

[7] T. Shisuma et al., Nucl. Instr. Meth. A 737, 170-175 (2014). 


\title{
High Flux Compton Gamma-ray Sources above $100 \mathrm{MeV}$
}

\author{
Vladimir N Litvinenko \\ Department of Physics and Astronomy, Stony Brook University
}

This talk will be dedicated to comparing various Compton sources capable of delivering gamma-ray beams with energies above pion threshold (e.g. to be specific, above 100 $\mathrm{MeV}$ ). Being photon-electron colliders Compton sources' performance depends on two colliding ingredients: electron and photon beams. Quality and quantity of electron beam depends on the accelerator: a linear (conventional or plasma), a storage ring or an ERL. Photon (laser) beams, and especially in high rep-rate Compton sources, strongly benefit from using intra-cavity collisions. Naturally, this benefit is especially high for long wavelength lasers, where ring-reflectivity low-loss optics is available. I will compare two photon sources: conventional IR and visible lasers with FELs. Finally, I will discuss "interaction region" designs and it effect on the flux and quality of the gamma-ray beam. 


\title{
EuroGammaS Gamma Beam Source delivery for ELI-NP Overview and Status
}

\author{
Kevin Cassou $^{1}$ on behalf of the EuroGammaS team ${ }^{2}$ \\ ${ }^{1}$ Laboratoire de l'Accélérateur Linéaire (LAL), CNRS Université Paris Saclay, Orsay, F-91405, \\ France \\ cassou@lal.in2p3.fr
}

${ }^{2}$ EuroGammaS: LNF-INFN (It), INFN Milano (It), INFN Ferrara (It), INFN Roma (It), INFN Catania(It), Comeb Srl (It), Università La Sapienza (It), Amplitudes Systemes and Technologies (Fr), Alsyom Sarl (Fr), Scandinova System (Sw), IN2P3 - CNRS (Fr).

The ELI Nuclear Physics Gamma Beam System (GBS) is an advanced Compton Gamma ray source with unprecedented performances of brilliance $\left(>10^{21}\right.$ photons $\left./ \mathrm{mm}^{2} / \mathrm{mrad}^{2} / \mathrm{s} / 0.1 \% \mathrm{BW}\right)$, monochromaticity $(0.5 \%)$ and energy tunability $(0.2-19 \mathrm{MeV})$ [1]. The GBS is being delivered and installed in Romania in the European Extreme Light Infrastructure for Nuclear Physics (ELINP).

The GBS is based on a $100 \mathrm{~Hz}$ repetition rate hybrid LINAC with an S-Band injector and two C-band accelerating sections, which delivers 32 electrons bunches [2], interacting with one high intensity visible laser pulse circulating in a non resonant laser beam cavity.

A full overview of the GBS machine will be given. The presentation will then focus on the laser optics parts of the GBS. The first section of the talk will be dedicated to the lasers and their transports integrated in the GBS to achieve the outstanding specifications of the GBS. A new scheme Ti:Sa laser photocahode laser is directly injected by optical master oscillator clock of the machine synchronization system delivering 32 subpicosecond laser pulses of $300 \mu \mathrm{J}$ in the UV leading to a average power of almost $1 \mathrm{~W}$. Two Yb:Yag based laser are delivering green laser picosecond pulses of $20 \mathrm{~W}$ and $40 \mathrm{~W}$ for the low energy and high energy interaction point respectively. A second part will give details of the laser beam circulator increasing the laser average power of the interaction from the tens of Watt to the kilowatt level [3]. The design will be illustrated. Assembly processes and high requirement on optics in terms of surface figure error and refection coating lead to specific developments that will be raised. A prototype laser beam circulator and associated test will be presented. The alignment and synchronization method will be reviewed

1. L. Serafini et al., "Technical Design Report: EuroGammaS proposal for the ELI-NP Gamma beam System", arXiv:1407.3669 (2014)

2. Bacci, A. et al., "Electron Linac design to drive bright Compton back-scattering gamma-ray sources ", Journal of Applied Physics, vol. 113,19, (2013)

3. K. Dupraz et al., "Design and optimization of a highly efficient optical multi pass system for $\gamma$-ray beam production from electron laser beam Compton scattering", Phys. Rev. STAB 17, 033501, (2014). 


\title{
High-flux, Ultra-narrow-bandwidth, Laser-Compton Light Source Architectures David J. Gibson
}

\author{
Lawrence Livermore National Laboratory, Livermore, CA, USA \\ gibson23@llnl.gov
}

Nuclear physics and engineering applications of gamma-rays generally require narrow bandwidth $\left(10^{-3}\right.$ or less $)$ beams and maximal flux $[1,2]$. A properly engineered laser-Comptonbased gamma-ray source provides the best opportunity for meeting both requirements. These sources inherently produce angle-correlated photons with an integrated bandwidth of $100 \%$. The details of the interaction geometry of each electron with each photon dictate the observed spectrum as a function of angle and the minimum bandwidth that may be obtained on axis [3].

Many of the applications of interest can benefit from an increase in average flux, rather than peak flux, so the issue becomes one of maximizing the beam current, along with the number of photons delivered to the interaction point. In pursuit of these goals, we have developed interaction laser systems based on a combination of commercial pump technology with custom pulse shaping systems to produce Joule-level picosecond beams [5] for interaction with bright electron beams. We have also developed pulse recirculation techniques optimized for these short, high energy pulses that relies on nonlinear optics to provide cavity switching with low b-integral accumulation [6]. To improve the beam flux, we have explored a path to creating trains of hundreds of electron bunches with spacing matched to the rf frequency $(11.424 \mathrm{GHz})$ of the accelerator, and have demonstrated the basic laser technology to drive an $\mathrm{rf}$ photogun to produce such beams [7].

Furthermore, while advanced, precision monochromators [4] have been demonstrated have have bandpasses of $10^{-5}$ or less, they have very narrow acceptance angles. A properly tailored gamma beam coupled with a monochromator has the potential to significantly improve the performance of such precision beams. The electron beams in an x-band accelerator naturally have a size on the order of $100 \mu \mathrm{m}$. Although it reduces the total flux, leaving this beam unfocused during the interaction will significantly reduce the angle/energy mixing in the beam and provide a naturally collimated, narrow-bandwidth source well suited for crystal monochromators. With a larger focal spot size, the focal region of the interaction laser is then much longer, and it makes sense to use a ns-scale laser to scatter off the electron bunch train. This greatly simplifies the laser architecture, and makes it easier to recover lost flux by increasing the total delivered laser energy.

Here we present an overview of novel, laser-Compton architectures along with the design of our currently-operating, laser-Compton x-ray source, and paths to extension of this technology to a wide range of nuclear physics and engineering applications.

This work was performed under the auspices of the U.S. Department of Energy by Lawrence Livermore National Laboratory under Contract DE-AC52-07NA27344.

1. D. Habs, T. Tajima, J. Schreiber, C. P. J. Barty, M. Fujiwara, and P. G. Thirolf, "Vision of nuclear physics with photo-nuclear reactions by laser-driven gamma beams," European Physical Journal D 55, 279-285. (2009) 
2. J. Pruet, D. P. McNabb, C. A. Hagmann, F. V. Hartemann, and C. P. J. Barty, "Detecting clandestine material with nuclear resonance fluorescence," Journal of Applied Physics 99, 123102-123101-123111 (2006)

3. F. V. Hartemann, D. J. Gibson, S. G. Anderson, A. M. Tremaine, P. T. Springer, A. J. Wootton, E. P. Hartouni, and C. P. J. Barty, "High-energy scaling of Compton scattering light sources," Physical Review Special Topics-Accelerators and Beams 8, 100702(100717) (2005)

4. M. Jentschel et al., "GAMS5", Journal of Research of the National Institute of Standards and Technology, Vol. 105, 167 (2000)

5. D. J. Gibson, C. A. Ebbers, F. Albert, A. Bayramian, S. M. Betts, F. V. Hartemann, R. A. Marsh, M. J. Messerly, H. Phan, S. S. Q. Wu, C. W. Siders and C. P. J. Barty."Advanced Laser Systems for MEGa-ray-based Nuclear Materials Detection and Assay." Proceedings of the 52nd Annual Meeting of the Institute for Nuclear Materials Management Vol 3. 2683-2689. (2011)

6. M. Y. Shverdin, I. Jovanovic, V. A. Semenov, S. M. Betts, C. Brown, D. J. Gibson, R. M. Shuttlesworth, F. V. Hartemann, C. W. Siders and C. P. J. Barty. "High-power picosecond laser pulse recirculation." Optics Letters 35(13): 2224-2226. (2010)

7. M. A. Prantil, E. Cormier, J. W. Dawson, D. J. Gibson, M. J. Messerly and C. P. J. Barty. "Widely tunable $11 \mathrm{GHz}$ femtosecond fiber laser based on a nonmode-locked source." Optics Letters 38(17): 3216-3218 (2013) 


\section{Monday, October 17, 2016 \\ Photo-nuclear Physics: 16:25 18:20 \\ Session Chair: M. Jentschel}




\title{
Photonuclear spectroscopy of discrete quantum states: basic principles, opportunities, and
} limitations

\author{
N. Pietralla* \\ IKP TU Darmstadt, Darmstadt, Germany \\ pietralla@ikp.tu-darmstadt.de
}

Nuclear and Elementary Particle Physics traditionally address the search for and research on the very fundamental building blocks of matter, their structure and their interactions. Research on the internal structure of atomic nuclei is complicated by the fact that the nuclear force between nucleons is not precisely known because it arises as the non-perturbative low-energy limit of the interaction between fundamental quarks and gluons, themselves forming the nucleons. Purely electromagnetic interactions of photons with atomic nuclei play a special role for nuclear structure physics as they allow for a model-independent separation between nuclear structure phenomena and details of the reaction mechanism because the electromagnetic interaction is precisely known.

Consequently, photonuclear reactions have been used in nuclear structure research for more than fifty years [1]. The study of photonuclear reactions requires, of course, the availability of photon beams in the relevant wavelength regime, i.e., gamma-ray beams in the $\mathrm{MeV}$ range. With the advent of accelerators for intense electron beams in the 1970s the bremsstrahlung process has widely been used for the production of gamma-ray beams, albeit with poor energy definition. Later-on, the process of Laser-Compton Backscattering has been demonstrated to provide energy-tunable, fully polarized gamma-ray beams with narrow band width and sufficient intensity for photonuclear applications.

We will discuss experimental approaches to photonuclear reactions and results of nuclear structure research obtained with them [2-5]. Recent data from the observation of Nuclear Resonance Fluorescence processes at the bremsstrahlung scattering site of the superconducting Darmstadt linear electron accelerator S-DALINAC at TU Darmstadt and at the High Intensity $\gamma-$ ray Source $\mathrm{HI} \gamma \mathrm{S}$ at Duke University will be discussed, e.g., [6-8]. An introductory outlook will be dared to what spectroscopy data will be in reach at the next-generation facilities for highintensity gamma-ray beams.

[1] F.R. Metzger, Prog. Nucl. Phys. 1, 53 (1959).

[2] U. Kneissl, N. Pietralla, A. Zilges, J. Phys. G 32, R217 (2006).

[3] N. Pietralla et al., Phys. Rev. Lett. 88, 012502 (2002).

[4] H.R. Weller et al.,, Prog. Part. Nucl. Phys. 62 (2009).

[5] D. Savran, T. Aumann, A. Zilges, Prog. Part. Nucl. Phys. 70 (2013).

[6] H. Pai et al., Phys. Rev. C 88, 054316 (2013); 93, 014318 (2016).

[7] J. Beller et al., Phys. Lett. B 741, 128 (2015).

[8] C Romig et al., Phys. Lett. B 744, 369 (2015).

This work was supported by research grants SFB 634 and SFB 1245 of the Deutsche Forschungsgemeinschaft and by the German BMBF under grant No. 05P15RDEN1. 


\title{
Origin of Dipole Strength in Atomic Nuclei
}

\author{
A. Zilges \\ University of Cologne, Germany \\ zilges@ikp.uni-koeln.de
}

The electric and magnetic dipole strength distribution in atomic nuclei shows a variety of structural features from the lowest energies to the region of the particle thresholds. A detailed knowledge of the structure of these dipole excitations will help to tackle challenging questions in various fields ranging from fundamental physics and astrophysics to applications [1-3].

Photons from bremsstrahlung sources and laser Compton backscattering are ideal tools for the investigation of dipole excitations. The latter offers the advantage of a very selective population of certain excitations and the possibility of a sensitive observation of the subsequent decay. Additional experiments using hadronic probes complete the knowledge obtained from electromagnetic probes.

The talk will give an overview about our present knowledge of the dipole strength up to the threshold and compare the different experimental techniques.

1. U. Kneissl, H.H. Pitz, A. Zilges, "Investigation of Nuclear Structure by Resonance Fluorescence Scattering”, Prog. Part. Nucl. Phys., 37, 349 (1996).

2. U. Kneissl, N. Pietralla, A. Zilges, "Low-lying diple modes in vibrational nuclei studied by photon scattering", J. Phys. G, 32, R217 (2006).

3. D. Savran, T. Aumann, A. Zilges, "Experimental Studies of the Pygmy Dipole Resonance", Prog. Part. Nucl. Phys., 70, 210 (2013). 


\title{
Nuclear excitations on the $\mathrm{keV}$ and $\mathrm{MeV}$ energy scale
}

\author{
Jonas Gunst ${ }^{1}$, Christoph H. Keitel ${ }^{1}$, Hans A. Weidenmüller ${ }^{1}$, Adriana Pálffy ${ }^{1}$ \\ ${ }^{1}$ Max-Planck-Institut für Kernphysik, Heidelberg, Germany \\ jonas.gunst@mpi-hd.mpg.de,keitel@mpi-hd.mpg.de,hans.weidenmueller@mpi-hd.mpg.de, \\ palffy@mpi-hd.mpg.de
}

Nuclear excitations cover a broad energy spectrum ranging from low-lying transitions in the $\mathrm{keV}$ region to high-energetic reactions involving nucleon emissions in the $\mathrm{MeV}$ range. In order to better understand nuclear structure and reactions it is of fundamental importance to study the light-nucleus interaction in both regimes.

In the first part, we treat the resonant interaction between x-rays and low-lying nuclear transitions in solid-state targets. In particular, the recently operational x-ray free electron lasers are expected to strongly promote this kind of interaction, driving nuclear experiments from single to multiple excitations per laser pulse. However, the highly intense x-ray beams not only amplify the direct nuclear photoexcitation, but also the electronic ionization of the target. New states of matter like cold, high-density plasmas can arise [1], where secondary nuclear processes as for instance nuclear excitation by electron capture (NEEC) are rendered possible. Taking into account the plasma expansion by a hydrodynamic model, we show that in the case of ${ }^{93 \mathrm{~m}} \mathrm{Mo}$ isomer triggering the indirect NEEC channel even provides the major contribution to the nuclear excitation, while it is totally negligible for other nuclear species like ${ }^{57} \mathrm{Fe}[2,3]$. Considering the nuclear properties, laser parameters and plasma conditions we could finally obtain a set of qualitative criteria to identify the dominating excitation channel in nuclear experiments with the XFEL [3].

In the higher frequency regime, new petawatt optical laser facilities such as the Extreme Light Infrastructure (ELI) hold promise to deliver in the near future coherent gamma-ray pulses, strongly enhancing laser-induced nuclear reactions. Considering the quasiadiabatic interaction regime, we show that a compound nucleus with an excitation energy several hundreds $\mathrm{MeV}$ above yrast can be formed, opening a so far totally unexplored territory $[4,5]$.

1. S. M. Vinko et al., Nature 482, 59 (2012).

2. J. Gunst, Yu. A. Litvinov, C. H. Keitel and A. Pálffy, Phys. Rev. Lett. 112, 082501 (2014).

3. J. Gunst, Y. Wu, N. Kumar, C. H. Keitel and A. Pálffy, Phys. Plasmas 22, 112706 (2015).

4. A. Pálffy and H. A. Weidenmüller, Phys. Rev. Lett. 112, 192502 (2014).

5. A. Pálffy, O. Buss, A. Hoefer and H. A. Weidenmüller, Phys. Rev. C 92, 044619 (2015). 


\title{
Exploring polarizabilities with the MAMI A2 tagged photon beam \\ Evangeline J. Downie ${ }^{1}$
}

\author{
${ }^{1}$ George Washington University: Washington DC, USA \\ edownie@gwu.edu \\ On behalf of the A2 Collaboration
}

Polarizabilities are fundamental properties of the nucleon which can be accessed by measuring the differential cross section, and singly and doubly polarized asymmetries in real Compton scattering (RCS). Their measurement gives rise to a better understanding of nucleon dynamics and structure. Polarizabilities play an important role in many areas of physics: from being the largest component of the theory error in the extraction of the radius of the proton from the Lamb shift, to influencing neutron star physics. Despite their all-pervading nature, and great theoretical interest, there is still a large uncertainty in the nucleon scalar polarizabilities, and the nucleon spin polarizabilities had, until recently, never been individually measured.

In the A2 Collaboration, at the MAMI (MAinzer MIkrotron) accelerator in Mainz, Germany, polarizabilities are accessed using the study of Compton scattering. The tagged photon beam, converted and tagged from the MAMI electron beam via the bremsstrahlung process in a thin radiator and the Glasgow Photon Tagging Spectrometer, impinges on a variety of targets to produce Compton scattered photons. The reaction products are then detected in the Crustal BallTAPS detector array, which covers almost the full $4 \pi$ rage. The large-angle spectrometer, coupled with a rage of polarized and unpolarized targets, enables good background suppression and high efficiency sampling of this low cross-section process.

The data are then fit using a variety of models to extract both scalar and spin polarizabilities with unprecedented precision. This report will cover the ongoing experimental series, investigating both event selection methodology and new experimental apparatus developed to enable higher precision. It will also cover the ongoing fitting studies used to optimize the extraction of the polarizabilities frim the data, and to guide the next generation of experiments. 


\title{
Photon Scattering from Nucleons at HI $\gamma \mathrm{S}$ at Medium Energies *
}

\author{
Mohammad W. Ahmed \\ North Carolina Central University, Triangle Universities Nuclear Laboratory, and Duke \\ University, Durham, NC 27708 USA \\ ahmed@tunl.duke.edu
}

Real-photon scattering is a viable tool to study electromagnetic (EM) and spin structure of nucleons. In this framework of nucleon Compton scattering, polarizabilities are fundamental quantities related to the nucleon structure. In the recent decade, effective field theories (EFT) have successfully established a bridge between the quantum chromodynamics (QCD) and lowenergy description of the nucleon and made predictions of the polarizabilities. Effective-lattice (L-EFT) calculations are also eminent on electromagnetic polarizabilities. High precision data are now needed to validate these predictions. The High Intensity Gamma-ray Source $(\mathrm{HI} \gamma \mathrm{S})$ at the Triangle Universities Nuclear Laboratory (TUNL) provides intense polarized photon beams that enable research on a variety of targets to extract EM and spin polarizabilities. During the last year, data have been collected using medium-energy and circularly polarized photon beams between 65 and $85 \mathrm{MeV}$ incident on Helium and Deuterium targets. These measurements constitute the highest precision data set available on Compton scattering from these nuclei. Measurement of cross section and asymmetries using linear polarized photons at $85 \mathrm{MeV}$, and circularly polarized photons at higher energies of $110 \mathrm{MeV}$ using Liquid He-3 target are forthcoming. These combined measurements constitute a program unmatched elsewhere and will provide high precision and stringent tests of the EFTs by establishing the fundamental nucleon structure constants in the low-to-medium energy region.

In this talk, I will present an overview of the Compton scattering program at HIGS at medium energies. Recent results, current activities and plans for new experiments will be summarized.

\footnotetext{
*This work is supported in part by the U.S. Department of Energy Office of Nuclear Physics under grants no. DEFG02-97ER41033 (TUNL), DE-SC000536, and DE-FG02-06ER41422 (GWU).
} 


\section{Monday, October 17, 2016 Future Gamma Systems Round Table hosted by TUNL: 20:30 21:30}




\section{Page Intentionally Left Blank}

Proc. of SPIE Vol. 10419 1041901-48

Downloaded From: https://www.spiedigitallibrary.org/conference-proceedings-of-spie on 26 Apr 2023 Terms of Use: https://www.spiedigitallibrary.org/terms-of-use 


\section{Tuesday, October 18, 2016 \\ Extreme Lasers and Applications: 8:30 10:25 \\ Session Chair: E. Liang}




\title{
Status of Laser-Driven Nuclear Fusion at LLNL ${ }^{*}$
}

\author{
Nathan Meezan ${ }^{1}$ \\ ${ }^{1}$ Lawrence Livermore National Laboratory, Livermore, California, USA
}

This talk reviews scientific results from the pursuit of ignition on the National Ignition Facility (NIF) and describes the program's forward looking research directions. Inertial Confinement Fusion (ICF) is a grand challenge with the potential to open new frontiers in the study of matter at extreme density, temperature, and pressure. In laser-driven indirect-drive ICF, laser beams heat an $\mathrm{x}$-ray enclosure called a hohlraum that surrounds a spherical pellet. The $\mathrm{x}$-ray radiation ablates the surface of the pellet, imploding a thin shell of deuterium/tritium (DT). The DT layer must accelerate to high velocity $(\mathrm{v}>350 \mathrm{~km} / \mathrm{s})$ and compress by a factor of several thousand. Since 2009, substantial progress has been made in understanding the major challenges to ignition: Rayleigh Taylor (RT) instability seeded by target imperfections and low-mode asymmetries seeded by systematic and random perturbations in the hohlraum x-ray drive, particularly from laser-plasma instabilities (LPI). Requirements on velocity, symmetry, compression, and stability have been demonstrated separately but not simultaneously. We now know that the RT instability, seeded mainly by the capsule support tent, severely degraded DT implosions from 2009-2012. Experiments using a "high-foot" drive with demonstrated lower RT growth improved the thermonuclear yield by a factor of 10, resulting in yield amplification due to alpha particle heating by more than a factor of 2 . However, large time dependent drive asymmetry in the LPI-dominated hohlraums remains unchanged, preventing further improvements. High fidelity 3D hydrodynamic calculations now explain these results. Future research focuses on: improved capsule mounting techniques; hohlraums with little LPI and controllable symmetry; and lower convergence implosions to better understand the physics of alpha heating. We are also pursuing several novel diagnostics techniques using nuclear particles to better understand ICF implosions and to further our understanding of nuclear science. We are confident that this approach, including a diverse portfolio of experimental, theoretical, and computational physics efforts by teams from around the world, will lead to further advances in solving the challenges of ICF.

*This work was performed under the auspices of the U.S. Department of Energy by Lawrence Livermore National Laboratory under Contract DE-AC52-07NA27344. 


\title{
High-Peak-Power Laser Research at the Laboratory for Laser Energetics and the Pathway to a 100-Petawatt Class Laser
}

\author{
E. M. Campbell, J. D. Zuegel, J. Bromage, S. G. Demos \\ Laboratory for Laser Energetics, Rochester, NY, USA \\ mcamp@lle.rochester.edu,zuegel@lle.rochester.edu,jbro@lle.rochester.edu, \\ sdemos@lle.rochester.edu
}

Numerous scientific opportunities in relativistic plasma physics, nuclear physics, and nonlinear quantum electrodynamics have been identified when on-target laser intensities approach or exceed $10^{24} \mathrm{~W} / \mathrm{cm}^{2}$. Systems that can achieve this peak power, contrast, and beam quality present significant laser, material, and experimental challenges. We will describe the vision and ongoing research at the Laboratory for Laser Energetics (LLE) that exploits the multikilojoule OMEGA EP laser to serve as a pump for an optical parametric amplifier line (EP OPAL). The resulting laser has the potential to achieve peak power and on-target intensities approaching $100 \mathrm{PW}$ and $10^{24} \mathrm{~W} / \mathrm{cm}^{2}$. Ongoing research from LLE's smaller ( 100-J) MultiTerawatt laser that will demonstrate much of the laser science and technology required for EP OPAL will also be presented.

This material is based upon work supported by the Department of Energy National Nuclear Security Administration under Award Number DE-NA0001944, the University of Rochester, and the New York State Energy Research and Development Authority. 


\title{
NOVEL EXAWATT TECHNOLOGIES AND THE PATH TO LASER INTENSITIES
} BEYOND $10^{24} \mathrm{~W} / \mathrm{CM}^{2}$

\author{
C. P. J. Barty ${ }^{1}$ \\ ${ }^{1}$ Lawrence Livermore National Laboratory, Livermore, CA, USA \\ barty1@1lnl.gov
}

Modern inertial confinement fusion lasers based on Nd:glass have amplification bandwidths that are capable of supporting pulses of less than a picosecond in duration. With the implementation of chirped pulse amplification (CPA), it is possible for beam lines at the National Ignition Facility at the Lawrence Livermore National Laboratory, the Laser Mega-Joule (LMJ) facility in Bordeaux, France, the LFEX laser at the Institute for Laser Engineering in Osaka, Japan and the Omega EP facility at the Laboratory for Laser Energetics in Rochester, New York to create petawatt peak power laser pulses of nominally $1-$ ps duration and 1-kJ energy [1]. While these systems are at the forefront of present highenergy, high-peak power capabilities, they utilize only a small fraction of the potential of the underlying Nd:glass laser amplification system and as such are very inefficient. A single beam line at the NIF, for example, has a stored energy in excess of $25 \mathrm{~kJ}$.

This presentation describes a new short pulse amplification architecture based on chirped "beams" [2], novel pulse compressors and existing beam phasing technologies that is capable of extracting the full, stored energy of a NIF or NIF-like beam line and in doing so produce from one beam line a near-diffraction-limited, laser pulse whose peak power would be in excess of 200 petawatts or 0.2 exawatts. This architecture is well suited to either low-f-number focusing or to multi-beam, dipole-focusing concepts [3]. With dipole focusing, it is anticipated that a single amplification beam line system will be capable of producing intensities in excess of $10^{26} \mathrm{~W} / \mathrm{cm}^{2}$ or more than 5 orders of magnitude beyond that possible from existing CPA based PW systems at NIF, LMJ, LFEX and Omega EP. At such intensities proton motion becomes relativistic during interactions with the laser pulse. Full extraction of beam line energy will also be enabling to full-scale demonstration of fast ignition concepts.

The novel amplification architecture described in this presentation is based entirely on existing technologies, proven optical damage performance and straightforward extensions of existing manufacturing technologies.

This work was performed under the auspices of the U.S. Department of Energy by the Lawrence Livermore National Laboratory under Contract DE-AC52-07NA27344.

[1] D. Zuegel, S. Borneis, C. P. J. Barty, B. Legarrec, C. Danson, N. Miyanaga, P. K. Rambo, C. Le Blanc, T. J. Kessler, A. W. Schmid, L. J. Waxer, J. H. Kelly, B. Kruschwitz, R. Jungquist, E. Moses, J. Britten, I. Jovanovic, J. Dawson, and N. Blanchot, "Laser challenges for fast ignition," Fusion Science and Technology 49, 453-482 (2006)

[2] C. P. J. Barty "Optical Chirped Beam Amplification and Propagation." US Patent \#6,804,045 B2, (2004) University of California.

[3] A.Gonoskov, A. Bashinov, I. Gonoskov, C. Harvey, A. Ilderton, A. Kim, M. Marklund, G. Mourou, and A. Sergeev Phys.Rev.Lett. 113, 014801 (2014) 


\section{APOLLON: present status and perspectives}

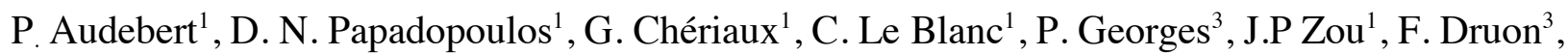
L. Martin ${ }^{1}$, A. Fréneaux ${ }^{1}$, A. Beluze ${ }^{1}$, N. Lebas ${ }^{1}$, J.M. Boudenne ${ }^{1}$, F. El Hai ${ }^{1}$, J. Prudent ${ }^{1}$, A.

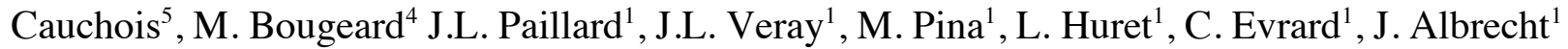

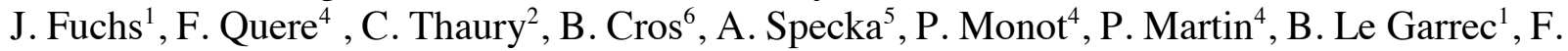
Mathieu $^{1}$, F. Amiranoff ${ }^{1}$ and F Hannachi ${ }^{7}$

${ }^{1}$ LULI, CNRS, Ecole Polytechnique, CEA, Univ. Pierre et Marie Curie, Palaiseau, France,

${ }^{2}$ LOA, ENSTA ParisTech, CNRS, Palaiseau, France,

${ }^{3}$ LCF, Institut d'Optique, CNRS, Univ Paris Sud, Palaiseau, France,

${ }^{4}$ LIDYL CEA,CNRS, Iramis, , Saclay, France

${ }^{5}$ LLR, CNRS, Ecole Polytechnique, Palaiseau, France,

${ }^{6}$ LPGP, Univ Paris Sud, Orsay, France,

${ }^{7}$ CENBG, CNRS, Univ Bordeaux, France, hannachi@ cenbg.in2p3.fr

High-intensity high-energy lasers are one of the best compact tools to concentrate in space and time and in a controllable manner, large amounts of energy. Consequently, a whole range of high-energy particles electrons, protons, highly charged ions, neutrons and radiations such as Xrays and gamma-rays can be produced as a result of their interactions with targets that can be either solid, liquid or gaseous [1-2].

The APOLLON laser system is a Ti:Sa based laser designed to deliver $150 \mathrm{~J}$ in 15 femtosecond pulses [3]. After focusing, intensities up to $2 \times 10^{22} \mathrm{~W} / \mathrm{cm}^{2}$ will be delivered to the experimentalists. This will allow reaching the so-called "ultra-relativistic regime" where both the electrons and ions are expected to be relativistic thus allowing for the exploration of novel matter properties. APOLLON laser with well-equipped experimental areas will be operated as a user's facility open to new national and international user communities. In a first stage, the facility will include four laser beams ( a 4 PW beam: $15 \mathrm{fs} \mathrm{/} 60 \mathrm{~J}, 1 \mathrm{PW}$ beam: $15 \mathrm{fs} / 15 \mathrm{~J}$, an uncompressed beam: $1 \mathrm{~ns} / 150 \mathrm{~J}$ and a probe beam: $20 \mathrm{fs} / 200 \mathrm{~mJ}$ ) which can be delivered with a repetition rate of one shot per minute in two experimental areas. It is designed to study highfields physics, laser-plasma electron and ion acceleration, X-ray and gamma-ray sources and their applications. The short-term experiments in both electron and ion production dedicated areas will be described and long-term perspectives will be outlined.

1. S.P.D. Mangles "An Overview of Recent Progress in Laser Wakefield Acceleration Experiments", Proceedings of the CAS-CERN Accelerator School: Plasma Wake Acceleration, Geneva, Switzerland, 23-29 November 2014, edited by B. Holzer, CERN2016-001 (CERN, Geneva, 2016)

2. A.M. Macchi et al, "Ion acceleration by superintense laser-plasma interaction", Rev Mod Phys 85 (2013) and references therein

3. G. Chériaux et al, "Apollon-10P: Status and implementation", AIP Conf. Proc. 1462, 78 (2012) 


\section{Novel Nuclear Applications of Laser-Driven Ion Acceleration}

Mamiko Nishiuchi ${ }^{1}$, Hironao Sakaki ${ }^{1}$, Timur Zh. Esirkepov ${ }^{1}$, Katsuhisa Nishio ${ }^{2}$, Tatiana A. Pikuz ${ }^{3}$, Anatoly Ya. Faenov ${ }^{3}$, Riccado Orlandi ${ }^{2}$, Alexander S. Pirozhkov ${ }^{1}$, Hiromitsu Kiriyama $^{1}$, Yuji Fukuda ${ }^{1}$, James Koga ${ }^{1}$, Masaki Kando ${ }^{1}$, Yukinobu Watanabe ${ }^{4}$, Sergei V. Bulanov ${ }^{1}$, Kiminori Kondo ${ }^{1}$, and Shoji Nagamiya ${ }^{5}$

${ }^{1}$ Kansai Photon Science Institute, National Institutes for Quantum and Radiological Science and Technology, 8-1-7 Umemidai, Kizugawa, Kyoto, 619-0215, Japan

mamiko.nishiuchi@qst.go.jp,sakaki.hironao@qst.go.jp,timur.esirkepov@qst.go.jp, pirozhkov.alexander@qst.go.jp,kiriyama.hiromitsu@qst.go.jp,fukuda.yuji@qst.go.jp,

koga.james@qst.go.jp,kando.masaki@qst.go.jp,bulanov.sergei@qst.go.jp, kondo.kiminori@qst.go.jp

${ }^{2}$ Japan Atomic Energy Agency, 8-1-7 Umemidai, Kizugawa, Kyoto 619-0215, Japan

nishio.katsuhisa@jaea.go.jp,orlandi.riccardo@jaea.go.jp

${ }^{3}$ Institute for Academic Initiatives, Osaka Uni., 1-1 Yamadaoka, Suita, Osaka 565-0871, Japan tatiana.pikuz@eie.eng.osaka-u.ac.jp,faenov.anatoly@photon.osaka-u.ac.jp,

${ }^{4}$ Interdisciplinary Graduate School of Engineering Sciences, Kyushu University, 6-1 Kasuga,

Fukuoka 816-8580, Japan

watanabe@aees.kyushu-u.ac.jp

${ }^{5}$ RIKEN, 2-1 Hirosawa, Wako, Saitama 351-0198, Japan

shoji.nagamiya@riken.jp

Decay rates and capture rates of unstable nuclei, especially short-lived and super-heavy ones, are necessary for understanding nucleosynthesis in stellar objects. Creation of such exotic nuclei and the measurement of their properties, however, is a formidable challenge for contemporary radiofrequency accelerator technology. A promising option is brought by present-day femtosecond petawatt lasers. Focused to matter in a micron spot these lasers produce a temporally quasi-static electric field with a strength exceeding $100 \mathrm{TV} / \mathrm{m}$ which efficiently strips and accelerates ions to tens and hundreds of $\mathrm{MeV} / \mathrm{u}$ over sub-micrometer distances. By combining state-of-the-art nuclear measurement techniques and a petawatt laser driven ion acceleration, we propose a novel application of the laser-driven ion acceleration scheme. The laser, focused to a target bombarded by an external ion beam, extracts the products of nuclear reactions and accelerates them to a few $\mathrm{GeV}^{1,2}$. This will enable measurements of the properties of yet unknown nuclei, including those with life-times well below 100 ns, and super-heavy nuclei.

Using a few hundred-terawatt laser focused onto a micron thick aluminum (Al) foil with a small amount of iron $(\mathrm{Fe})$ simulating nuclear reaction products, we experimentally demonstrate an extraction of almost fully stripped Fe nuclei and their acceleration up to $0.9 \mathrm{GeV}$. We found that heavy ions, with a much larger than in conventional technology charge-to-mass ratio, can be obtained in the form of an energetic, low-emittance, high-density beam. Our findings may pave the way towards a new laser-driven source of exotic nuclei, which are at present inaccessible with conventional radio isotope facilities and heavy ion accelerators. The data obtained using this source can also facilitate a modeling of nuclear reaction processes in an accelerator-driven subcritical reactor, a failsafe alternative to common critical reactors.

1. M. Nishiuchi, et al., "Acceleration of highly charged GeV Fe ions from a low-Z substrate by intense femtosecond laser," Phys. of Plasmas, 22, 033107 (2015).

2. M. Nishiuchi, et al., "Towards a novel laser-driven method of exotic nuclei extraction acceleration for fundamental physics and technology," Plasma Physics Reports, 42, 327 (2016). 


\section{Tuesday, October 18, 2016 Nuclear Security Applications: 10:45 12:50 \\ Session Chair: R. Hajima}




\title{
Non-intrusive Inspection Technologies for Nuclear Security and Materials Assaying
}

\author{
Dr. Robert J. Ledoux \\ Passport Systems, Billerica, MA United States \\ Ledoux@passportsystems.com
}

There is a great need for new non-intrusive inspection technologies that provide substantially more information than conventional X-ray or neutron beam projective imaging technologies in the fields of Nuclear Security and Materials Identification. In recent years, a variety of techniques using high energy X-ray beams have been developed to provide nondestructive materials discrimination and identification. The physics behind these new inspection modalities will be presented. In many cases, a major step forward in the fields of application and performance of these new non-destructive assaying technologies for nuclear materials and contraband in general would be provided by the availability of tunable narrow energy band high energy X-ray sources. Examples of the present state-of-the-art nondestructive assaying technologies will be presented with emphasis on limitations posed by present broad energy X-ray beams and the significant improvements made possible by the monochromatic sources. The practical requirements of the next generation of electron and X-ray sources that enable new security applications will be discussed. 


\title{
Imaging in Active Interrogation: Comparison of Photon Beams
}

\author{
Anna Erickson ${ }^{1}$, Paul Rose ${ }^{1}$, Joe Harms ${ }^{1}$, Wesley Gillis ${ }^{1}$, Igor Jovanovic ${ }^{2}$, Jason Nattress ${ }^{2}$ \\ ${ }^{1}$ Georgia Institute of Technology, Atlanta, GA, USA, erickson@gatech.edu \\ ${ }^{2}$ University of Michigan, Ann Arbor, MI, USA
}

In cargo scanning for special nuclear materials, beam source and detector response influence output image quality, which ultimately determines whether special nuclear material (SNM) can be detected. While bremsstrahlung beams are industry standard [1], the spectrum is continuous and highly biased towards low energies resulting in low penetration capabilities and increased scan time and dose to ensure adequate detection statistics and image quality. Use of monoenergetic interrogation beams could lead to decreased dose and scan time and improved image quality and material determination. Low-energy nuclear reactions result in quasimonoenergetic beams, for example ${ }^{11} \mathrm{~B}(\mathrm{~d}, \mathrm{n}){ }^{12} \mathrm{C}$ produces prominent gamma peaks at 4.4 and 15.1 $\mathrm{MeV}$ [2]. Inverse Compton Scattering (ICS) is another technique which produces quasimonoenergetic photons and has the advantage of being tunable, allowing the user to select the beam energy [3]. In this work, we compare performance of the three imaging systems based on image quality and dose due to primary interrogating photons as well as secondary radiation.

We evaluate image quality based on the beam source used for both small-scale and largescale imaging. We create images which measure transmission (Figure 1) and $\mathrm{Z}_{\text {eff }}$ (Figure 2). For image quality assessment, we measure pixel error, root-mean-square error (RMSE). Additionally, for the large-scale image, we compare the contrast-to-noise ratio (CNR) across source type for nine materials. Pixel error measurement determines the confidence with which a system is able to confirm the presence of SNM. RMSE and CNR are commonly used to determine system sensitivity, allowing for a quantitative study of image quality.

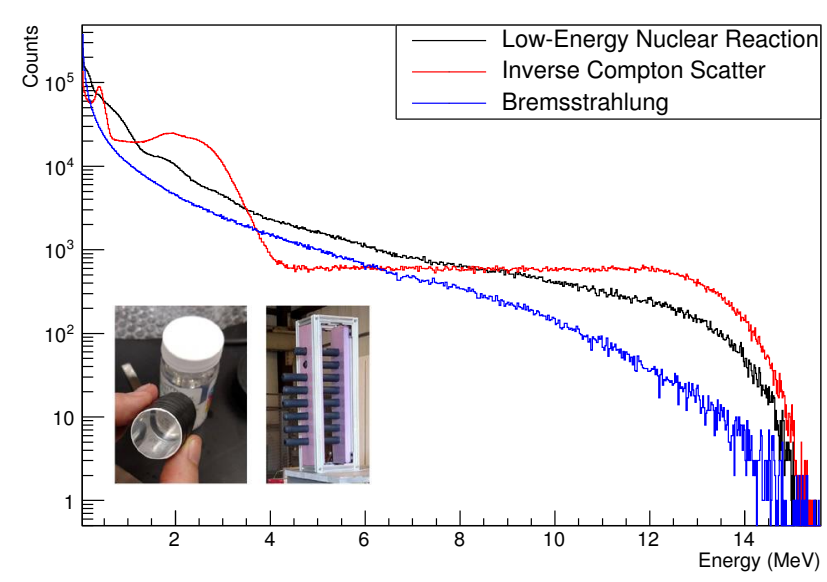

Figure 1: Response of Cherenkov detectors to various interrogating sources.

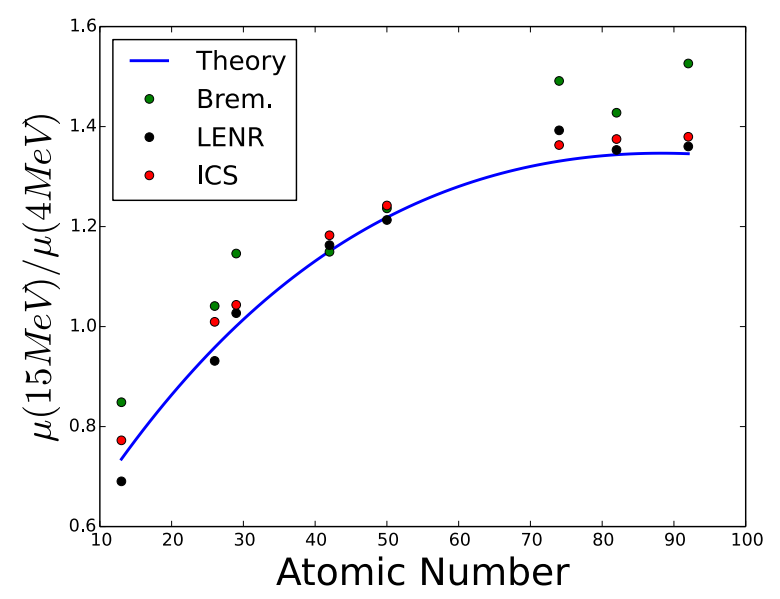

Figure 2: Comparison of three beams in $\mathrm{Z}_{\mathrm{eff}}$ analysis. 
The detectors used in this imaging array are quartz Cherenkov-based detectors [4]. The crude energy resolution of these detectors has been shown to be sufficient for $\mathrm{Z}_{\text {eff }}$ reconstruction [2]. We first validate our simulation results by comparing to previously published data [2] for the same imaging geometry. We then compare images created with each beam source, as shown in Figure 3. Finally, we calculate the total dose associated with each beam normalized by the image quality metrics.

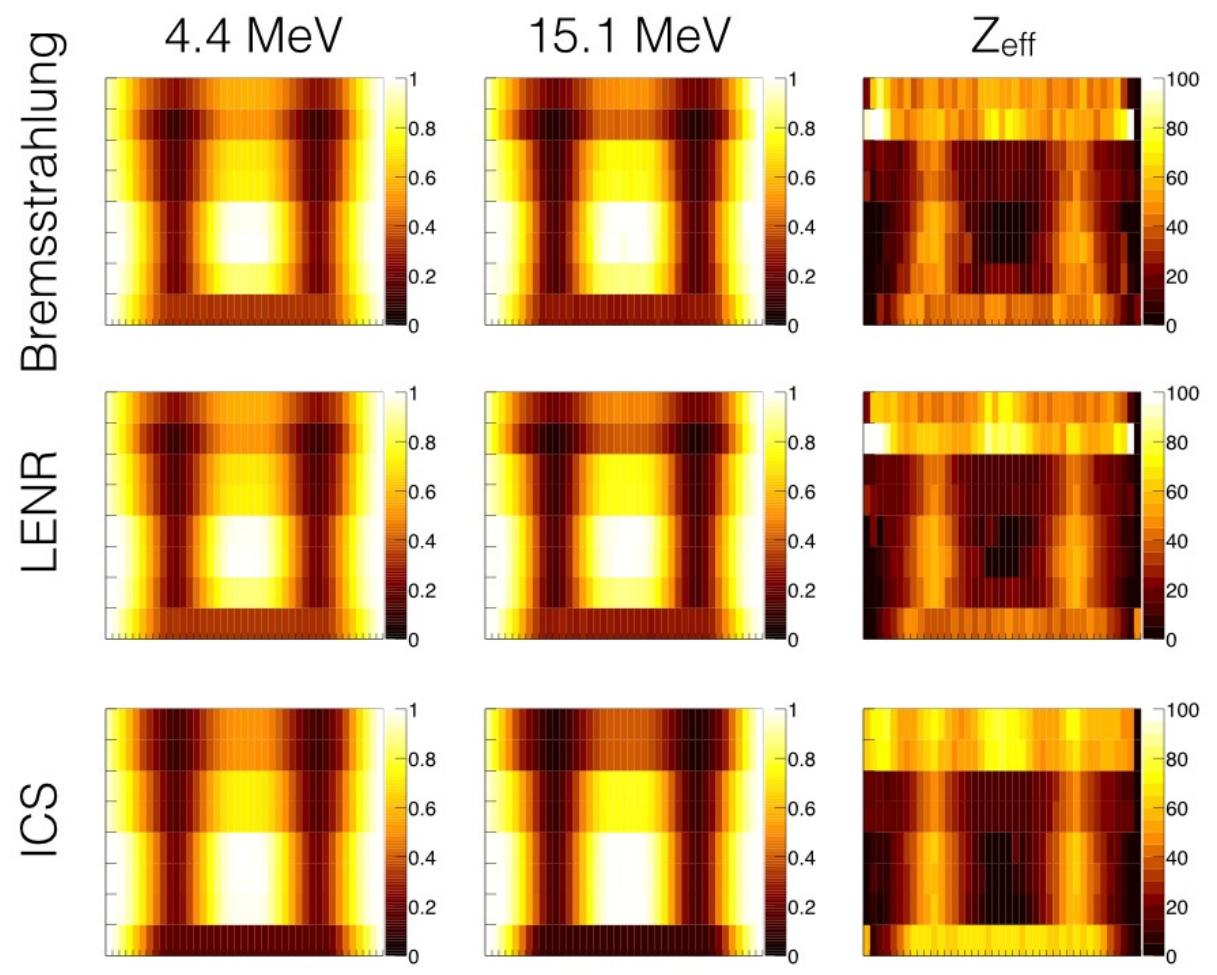

Figure 3. Total photon transmission at 4.4 MeV and 15.1 MeV for each imaging source studied and the map of $Z_{\text {eff }}$.

This work has been supported by the US Department of Homeland Security, Domestic Nuclear Detection Office, under competitively awarded grant 2015-DN-077-ARI096. This support does not constitute an express or implied endorsement on the part of the Government.

1. Quiter et al. "A method for high-resolution x-ray imaging of intermodal cargo containers for ssionable materials," J. Appl. Phys. 103, 064910 (2008).

2. P. Rose, A. Erickson, M. Mayer, J. Nattress, I. Jovanovic, "Uncovering Special Nuclear Materials by Low-energy Nuclear Reaction Imaging," Scientific Reports 6, 24388; doi: 10.1038/srep24388 (2016).

3. Banerjee, S. et al. "Compact source of narrowband and tunable X-rays for radiography," Nucl. Instrum. Meth. B 350, 106-111 (2015).

4. B. Rose Jr., A.S. Erickson, "Calibration of Cherenkov detectors for monoenergetic photon imaging in active interrogation applications", Nucl. Instrum. Meth. A 799, 99-104 (2015). 


\title{
Anticipations of NRF-based NDA of Nuclear Material Using Monochromatic Gamma-ray Beams
}

\author{
Michio Seya ${ }^{1}$, Ryoichi Hajima ${ }^{2}$, Takehito Hayakawa ${ }^{2}$, Mitsuo Koizumi ${ }^{1}$ \\ 1 Japan Atomic Energy Agency, \\ Tokai-mura, Ibaraki-ken, Japan \\ seya.michio@jaea.go.jp,koizumi.mitsuo@jaea.go.jp \\ 2 National Institute for Quantum and Radiological Science and Technology, \\ Tokai-mura, Ibaraki-ken, Japan \\ hajima.ryoichi@qst.go.jp,hayakawa.takehito@qst.go.jp
}

High-intensity monochromatic gamma-ray beams produced by inverse Compton scattering of laser beams (laser Compton scattering: LCS) with electron beams from a super conducting accelerator could be used as interrogation probe of NRF-based NDA systems in both of nuclear security and nuclear safeguards.

In the field of nuclear security, the NRF-base NDA systems would be used in the following ways.

-secure detection of nuclear material (NM) covered with thick shielding materials in the freight containers

-precise checking of interior structures of detected / suspicious objects

-substitute of highly radioactive gamma-ray sources like ${ }^{60} \mathrm{Co}$ and ${ }^{137} \mathrm{Cs}$ etc.

With combined use of X-ray scanning system, high-intensity monochromatic gamma-ray beam is very good interrogation prove for pin-point non-destructive detection of nuclear material with NRF in thick shielding material found by the X-ray scanning. It also could be used as prove of CT imaging for checking whether or not detected / suspicious objects have detonation system, for an example, inside them.

Presently in several countries like USA ${ }^{[1][2]}$, in order to reduce risk of RDD (radiological dispersion device), replacements of highly radioactive isotopes emitting gamma-rays for medical use and as radiation standards etc. with alternatives are under progressing. Especially strong radioactive source for radiation standards could be replaced with monochromatic gamma-rays produced by LCS.

In the field of nuclear safeguards, the NRF-base NDA systems would be used in the following ways.

-precise measurements of nuclear material in LWR spent fuel assemblies

-precise measurement of quantities of $\mathrm{U} / \mathrm{Pu}$ fissile isotopes in canisters of melted fuel debris

-precise measurement of quantities of minor actinide isotopes in $\operatorname{ADS}(*)$ fuels before and after irradiation in the ADS reactor core

(*) ADS (: accelerator driven sub-criticality system or simply accelerator driven system) is one of the transmutation systems of long half-life minor actinides (MA: Np, Am, Cm etc.) and fission products (FP) using a neutron source driven by a proton linear accelerator. 
The LWR spent fuel assemblies, melted fuel debris and ADS (spent) fuel assemblies have high gamma-ray and neutron radiations, which makes conventional passive NDA techniques difficult to be applied for quantification of nuclear material. Recently IAEA issued the report ${ }^{[3]}$ of long term R\&D for safeguards in which partial defect test (verification by NDA system) on spent fuel assembly prior to transfer to difficult to access storage. The NRF-base NDA system could be used for this purpose ${ }^{[4][5]}$.

JAEA has proposed the NRF-base NDA system for precise measurement of nuclear material in melted fuel debris in a canister ${ }^{[6]}$. The NRF-base NDA system also could be applied for precise measurement of NM/MA/FP in the ADS (spent) fuel assemblies.

In this presentation, we show the above anticipations of usages of NRF-based NDA of NM using monochromatic gamma-ray beams.

This work was supported by a Government (MEXT) Subsidy for Strengthening Nuclear Security.

[1] K. Hatcher (Office of Radiological Security) "NNSA Activities with experimental and commercial irradiation", (2015).

[2] G. M. Moore and M. A. Pomper, "Permanent Risk Reduction: A Roadmap for Replacing High-Risk Radioactive Sources and Materials", CNS Occasional Paper No. 23 (2015).

[3] STR_375_IAEA_Department_of_Safeguards_Long-Term_R\&D_Plan_2012-2023 (2013).

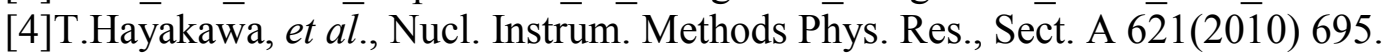

[5]T.Shizuma, et al., Nucl. Instrum. Methods Phys. Res., Sect. A 737(2014) 170.

[6] T. Shizuma, et al.; Proc. of ESARDA-37; 2015 


\title{
Active Interrogation by Nuclear Reaction-Based Sources and Particle Discriminating Detectors
}

\author{
Igor Jovanovic $^{1}$, Jason Nattress ${ }^{2}$, Anna Erickson ${ }^{2}$, Paul Rose ${ }^{2}$ \\ ${ }^{1}$ University of Michigan,Ann Arbor,MI,USA,ijov@umich.edu,jtnatt@umich.edu \\ ${ }^{2}$ Georgia Institute of Technology, Atlanta, GA, USA, erickson@gatech.edu, prose6@gatech.edu
}

Active interrogation (AI) is a recognized method that can enable detection of various materials and objects in a broad range of security scenarios. Of those, the detection of shielded special nuclear material (SNM) is one of the greatest challenges facing nuclear security, where success relies on the detection of small characteristic signals in relatively large, complex, and variable backgrounds. Achieving high sensitivity and specificity to SNM in practical measurements requires a unique and innovative approach that can also meet practical constraints, such as limited scan time and low radiation dose. Since the AI radiation sources and detectors work in tandem, their choice, design, and method of operation need to be considered in an integrated fashion.

We have been studying a unique AI approach that integrates the neutron and gamma transmission signatures to deduce characteristic material properties, including those that can be used to identify SNM. In our approach, the AI probe is a low-energy nuclear reaction, such as ${ }^{11} \mathrm{~B}(\mathrm{~d}, \mathrm{n}-\gamma){ }^{12} \mathrm{C}$, which can readily provide high-energy monoenergetic photons $(\gamma$ rays $)$ and quasimonoenergetic, tunable neutrons when driven by a compact ion source [1]. In our experiments, the $\gamma$ ray energies extend up to $15.1 \mathrm{MeV}$, while the neutron energies extend up to $16.5 \mathrm{MeV}$. We have developed novel Cherenkov and composite neutron detectors [2] and used them to perform $Z_{\text {eff }}$ resolved imaging [1] and delayed neutron detection (Fig. 1) [1,3] in the unique AI environment provided by this dual-particle source.

We are extending this approach by developing and deploying other detector types with a goal to perform dual-particle spectroscopy using a single, quasi-monoenergetic AI source and a single detector type. Pulse shape discriminating detectors based on nuclear recoil, such as liquid and plastic scintillators, are known to be able to reliably distinguish neutrons from $\gamma$ rays, and their characteristic light output distributions can be used to reconstruct the neutron and $\gamma$ spectra. In addition to light output distributions, the time of flight measurements can be used to reconstruct the neutron spectra.

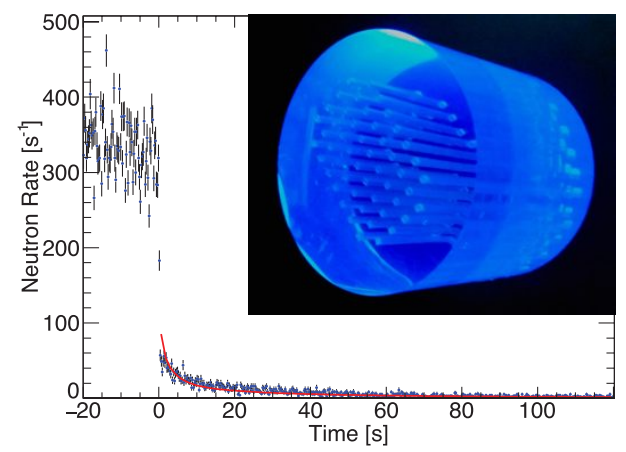

Figure 1: Delayed neutron signature from uranium fission [2] detected using a novel composite detector [3] (inset).
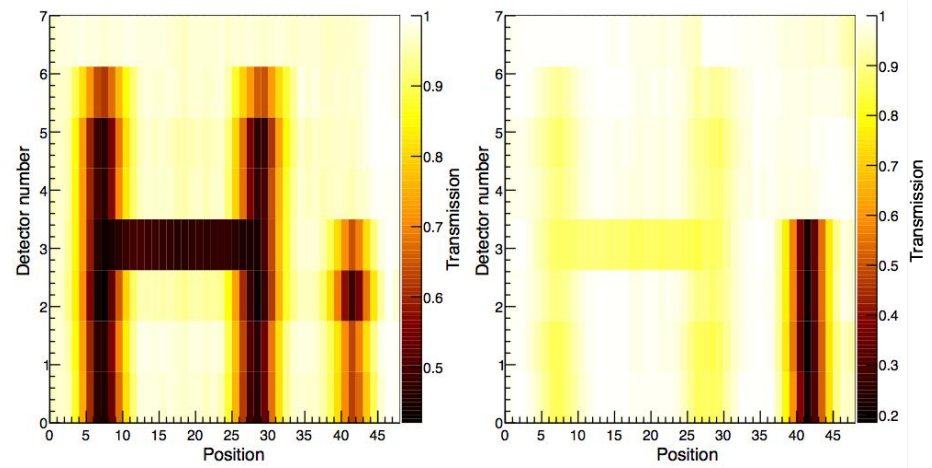

Figure 2: Neutron (left) and gamma (right) image of a test object. "H" - borated polyethylene; "I" - natural uranium 
Some preliminary results of dual-particle imaging with particle discriminating detectors are shown in Figs. 2-3. Specifically, we demonstrate the ability of this approach to selectively improve the image contrast for hydrogenous and high-Z materials (Fig. 2). We also demonstrate reconstruction of $Z_{\text {eff }}$ using $\gamma$ rays at $4.4 \mathrm{MeV}$ and $15.1 \mathrm{MeV}$ (Fig. 3), similar to that previously accomplished with Cherenkov detectors [1]. We show on the example of the $16.5 \mathrm{MeV}$ neutron transmission that light output distributions from liquid scintillators can also be used to deduce material composition (Fig. 3), noting that neutron transmission is intrinsically isotopically selective.
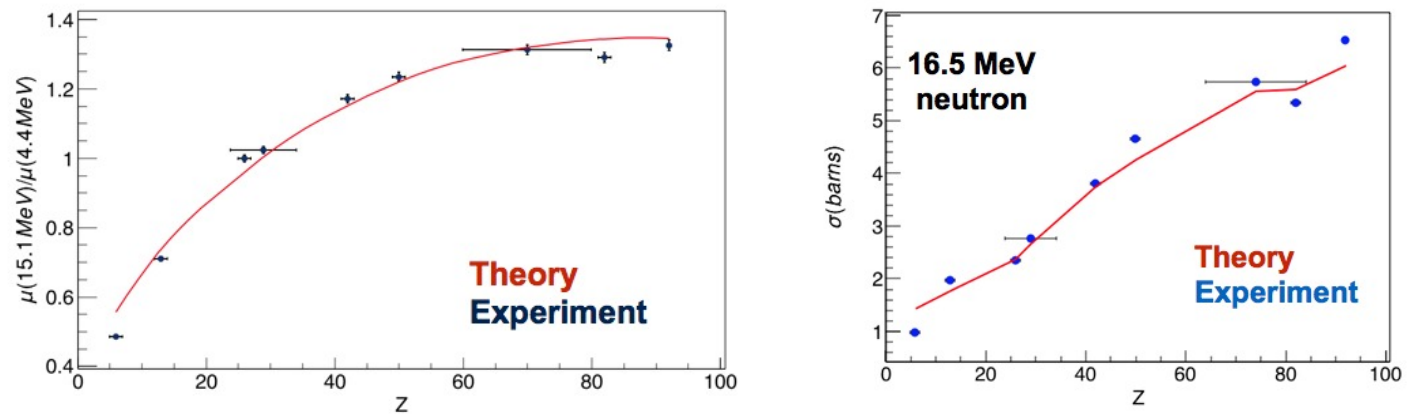

Figure 3: Left: reconstruction of $Z_{\text {eff }}$ using the differential $\gamma$ transmission (4.4 MeV and 15.1 MeV). Right: measured $16.5 \mathrm{MeV}$ neutron transmission is in good agreement with the test material cross section. Neutron and $\gamma$ measurements were performed simultaneously in liquid scintillation detector.

Combining the AI sources based on low-energy nuclear reactions and particle discriminating spectroscopic detectors offers new measurement modalities that could resolve ambiguities present when only a single radiation type is used. These approaches could be appropriately modified to accommodate other types of sources with unique characteristics, for example by driving the nuclear reaction sources by laser-produced ions or by driving the photoneutron production using laser-produced high-energy X-rays.

This work has been supported by the National Science Foundation under Grant No. ECCS1348366 and ECCS-1348328 and by the U.S. Department of Homeland Security under Grant Award Number 2014-DN-077-ARI079-02 and 2014-DN-077-ARI078-02.

1. P. Rose, A. Erickson, M. Mayer, J. Nattress, and I. Jovanovic, "Uncovering Special Nuclear Materials by Low-energy Nuclear Reaction Imaging," Scientific Reports, 6, 24388 (2016).

2. M. Mayer, J. Nattress, and I. Jovanovic, "Detection of Special Nuclear Material from Delayed Neutron Emission Induced by a Dual-Particle Monoenergetic Source," Applied Physics Letters, 108, 264102 (2016).

3. J. Nattress, M. Mayer, A. Foster, A. Meddeb, C. Trivelpiece, Z. Ounaies, and I. Jovanovic, "Capture-gated Spectroscopic Measurements of Monoenergetic Neutrons with a Composite Scintillation Detector", IEEE Transactions on Nuclear Science, 63, 1 (2016).

4. M. Mayer, J. Nattress, V. Kukharev, A. Foster, A. Meddeb, C. Trivelpiece, Z. Ounaies, and I. Jovanovic, "Development and Characterization of a Neutron Detector Based on a Lithium Glass-Polymer Composite", Nuclear Instruments and Methods in Physics Research $A$, 785, 117 (2015). 


\title{
Laser-driven Pulsed Neutron Sources as a Potential Pool-side Characterization Tool for Nuclear Fuels
}

\author{
Sven C. Vogel ${ }^{1}$, Adrian S. Losko ${ }^{1,2}$, Anton S. Tremsin ${ }^{3}$, \\ Mark A.M. Bourke ${ }^{1}$, Kenneth J. McClellan ${ }^{1}$ \\ ${ }^{1}$ Materials Science and Technology Division, Los Alamos National Laboratory, \\ Los Alamos, NM 87545, U.S.A. \\ sven@lanl.gov,alosko@lanl.gov,bourke@lanl.gov,kmcclellan@lanl.gov \\ ${ }^{2}$ Nuclear Engineering Department, University of California Berkeley, \\ Berkeley, CA 94720, U.S.A. \\ ${ }^{3}$ Space Science Laboratory, University of California Berkeley, Berkeley, CA 94720, U.S.A. \\ ast@ssl.berkeley.edu
}

The unique advantages of neutrons for characterization of nuclear fuel materials [1] are applied at the pulsed spallation neutron source at LANSCE to accelerate the development and ultimately licensing of new nuclear fuel forms. Neutrons allow to characterize the crystallography of phases consisting of heavy elements (e.g. uranium) and light elements (e.g. oxygen, nitrogen, or silicon) [2]. The penetration ability in combination with comparably large (e.g. cm sized) beam spots provide microstructural characterization of typical fuel geometries for phase composition, strains, and textures from neutron diffraction.

In parallel, we are developing energy-resolved neutron imaging and tomography with which we can complement diffraction characterization. This unique approach not only allows to visualize cracks, arrangement of fuel pellets in rodlets etc., but also characterization of isotope or element densities by means of neutron absorption resonance analysis [3].

Laser-driven pulsed neutron sources [4] have the potential to provide these capabilities "pool-side", e.g. at the Advanced Test Reactor at Idaho National Laboratory. Compared to proton accelerator driven spallation sources, requiring investments exceeding $\$ 1 \mathrm{~B}$, the investment cost for a laser-driven neutron source would be of the order of several \$10M with the potential of similar flux to that of a smaller, earlier generation spallation neutron source. Compared to electron accelerator-driven neutron sources, the flux of a laser-driven source would be at least one order of magnitude higher. Compared to reactor neutron sources, the pulse structure of the laser-driven neutron source would enable unique characterization not possible with steady-state reactor neutrons.

In this presentation, we provide an overview of our recent accomplishments in fuel characterization for accident-tolerant fuel consisting of uranium nitride/uranium silicide composite fuels as well as metallic fuels. We will further discuss recent results demonstrating the use of laser-driven neutron sources for these efforts.

1. S.C. Vogel, "A Review of Neutron Scattering Applications to Nuclear Materials", ISRN Materials Science, 2013, Article ID 302408 (2013).

2. H. Matthias Reiche, Sven C. Vogel, Ming Tang, "In situ synthesis and characterization of uranium carbide using high temperature neutron diffraction", Journal of Nuclear Materials, 471 308-316 (2016).

3. A.S. Tremsin, S.C. Vogel, M. Mocko, M.A.M. Bourke, V. Yuan, R.O. Nelson, D.W. Brown, W.B. Feller, "Non-destructive studies of fuel pellets by neutron resonance absorption radiography and thermal neutron radiography", Journal of Nuclear Materials 440 633-646 (2013).

4. M. Roth et al., "Bright Laser-Driven Neutron Source Based on the Relativistic Transparency of Solids", Physical Review Letters 110, 044802 (2013). 


\section{Page Intentionally Left Blank}

Proc. of SPIE Vol. 10419 1041901-64

Downloaded From: https://www.spiedigitallibrary.org/conference-proceedings-of-spie on 26 Apr 2023 Terms of Use: https://www.spiedigitallibrary.org/terms-of-use 


\section{Tuesday, October 18, 2016 \\ High Energy Photon Systems \& Applications: 14:00 16:30 \\ Session Chair: Y. Wu}




\section{PERLE - a powerful ERL facility concept}

Erk Jensen $^{1}$, A. Bogacz ${ }^{2}$, O. Brüning ${ }^{1}$, R. Calaga ${ }^{1}$, V. Chetvertkova ${ }^{1}$, M. Klein ${ }^{3}$, A. Milanese ${ }^{1}$, D. Pellegrini ${ }^{1}$, A. Stocchi ${ }^{4}$, A. Valloni ${ }^{1}$, D. Wollmann ${ }^{1}$, F. Zomer ${ }^{4}$

${ }^{1}$ CERN, Geneva, Switzerland

Erk.Jensen@cern.ch

${ }^{2}$ Jefferson Lab, Newport News, VA, USA,

${ }^{3}$ University of Liverpool, $U K$

${ }^{4} L A L$, CNRS-in2p3, Orsay, France

A $60 \mathrm{GeV}$ energy recovery linac (ERL) is needed for a possible extension of the LHC towards a hadron-electron collider (LHeC [1]) or for the hadron-electron version of the FCC [2]. An ERL test facility was initially proposed to validate design choices, notably the high current, $\mathrm{CW}$, multi-pass operation. The resulting facility is novel, complementary to existing or planned facilities elsewhere and turns out powerful to perform a number of experiments in different fields reaching from unique tests of accelerator components via elastic ep scattering to laser-Compton backscattering for photon physics - this is why the name PERLE (Powerful ERL for Experiments) was chosen. It operates with superconducting cavities at $800 \mathrm{MHz}$, features FMC cell based, vertically stacked return arcs and a high-current, $5 \mathrm{MeV}$ photoinjector. In up to 3 recirculation passages up to $900 \mathrm{MeV}$ beam energy can be reached with beam currents in excess of $10 \mathrm{~mA}$.

1. J.L. Abelleira Fernandez et al., "A Large Hadron Electron Collider at CERN - Report on the Physics and Design Concepts for Machine and Detector, Journal of Physics G: Nuclear and Particle Physics, vol. 39, no. 7, 2012.

2. M. Benedikt and F. Zimmermann, "Status and Challenges of the Future Circular Collider Study," $12^{\text {th }}$ International Topical Meeting on Nuclear Applications of Accelerators, Washington, DCF, USA, 2015 


\title{
NewSUBARU Gamma Beam Source - Status and Activities
}

\author{
Shuji Miyamoto \\ ${ }^{1}$ Laboratory of Advanced Science and Technology for Industry, \\ University of Hyogo, Kouto, Hyogo, JAPAN \\ miyamoto@lasti.u-hyogo.ac.jp
}

The laser Compton scattering (LCS) $\gamma$-rays have advantages that an energy tunable quasimonochromatic and an almost 100\% linearly (circularly) polarized $\gamma$-ray beam source. The polarized $\gamma$-ray beams are powerful tools to study the nuclear physics and a material science such as measurements of transition strengths with parity assignments, a magnetic Compton scattering, and polarized electron and positron generation. The $(\gamma, n)$ reactions with linearly polarized beam have not been studied well, since the $100 \%$ linear polarized photon beam has not been practically used before the developments of the LCS $\gamma$-ray facilities.

The synchrotron light facility NewSUBARU [1] is operated by the LASTI, University of Hyogo. The LCS $\gamma$-ray beam-line BL01 [2] with small shielding hutch-1 was started to operate from 2005 using the $0.5-1.5 \mathrm{GeV}$ electron beams in the NewSUBARU storage ring. Lasers with different wavelengths are used to produce the LCS photon beam in the energy range from 0.5 $\mathrm{MeV}$ to $76 \mathrm{MeV}$. A experimental hutch, GACKO [3] (Gamma Collaboration Hutch of Konan University), was added to use at the BL01 in 2012.

Recently, we have measured the photo neutron distribution emitted from the interaction between linearly polarized gamma-rays and nuclei $[4,5]$. This was the first demonstration of a theory of photo-neutron emission which depend on the polarization.

This work was done by collaboration with QST, JAEA, Konan University, Osaka University, Osaka Prefecture University, Kyoto University, Ecole Polytechnique, JASRI, RIKEN, KEK and SPring-8 team.

1. A.Ando, S.Amano, S.Hashimoto, H.Kinoshita, S.Miyamoto, T.Mochizuki, M.Niibe, Y.Shoji, M.Terasawa, T.Watanabe and N.Kumagai, J. Synchrotron Rad., 5, pp.342-344 (1998). http://www.lasti.u-hyogo.ac.jp/NS-en/

2. S.Miyamoto, Y.Asano, S.Amano, D.Li, K.Imasaki, H.Kinugasa, Y.Shoji, T.Takagi, and T.Mochizuki, "Laser Compton back-scattering gamma-ray beamline on NewSUBARU", Radiation Measurements, 41, pp.S179-S185(2007).

3. H.Utsunomiya, S.Hashimoto, S.Miyamoto , "The $\gamma$-Ray Beam-Line at NewSUBARU", Nuclear Physics News, 25, Issue 3, pp.25-29, July-September (2015).

4. K.Horikawa, S.Miyamoto, T.Mochizuki, S.Amano, D.Li, K.Imsakaki, Y.Izawa, K.Ogata, S.Chiba, T.Hayakawa, "Neutron angular distribution in $(\gamma, n)$ reactions with linearly polarized $\gamma$-ray beam generated by laser Compton scattering ", Physics Letters B, 737, pp.109-113 (2014).

5. T. Hayakawa, T. Shizuma, S. Miyamoto, S. Amano, A. Takemoto, M. Yamaguchi, and K. Horikawa, H. Akimune, K. Ogata and M. Fujiwara, "Spatial anisotropy of neutrons emitted from the $56 \mathrm{Fe}(\gamma, \mathrm{n}) 55 \mathrm{Fe}$ reaction with a linearly polarized $\gamma$-ray beam ", Physical Review $\mathrm{C}$, 93, pp. 004313-1-4 (2016). 


\title{
Gamma-ray Source Activities at SINAP
}

\author{
Jianhui Chen ${ }^{1}$, Hailong $\mathrm{Wu}^{1}$, Bo Liu ${ }^{1}$, Dong $\mathrm{Wang}^{1}$, Zhentang Zhao ${ }^{1}$ \\ ${ }^{1}$ Shanghai Institute of Applied Physics, Shanghai, China \\ chenjianhui@sinap.ac.cn
}

High brightness $\gamma$-rays produced by laser Compton scattering (LCS) are ideal probes for the study of nucleon and nuclear structure. In Shanghai Institute of Applied Physics (SINAP), a storage-ring based $\gamma$-rays source, so-called Shanghai Laser-Electron Gamma-ray Source (SLEGS) at the Shanghai Synchrotron Radiation Facility (SSRF), has recently been approved and will start construction within a year.

We also have proposed such a $\gamma$-ray source using the backscattering of a laser from the bright electron beam produced by the linac of the Shanghai Soft X-ray Free-electron Laser (SXFEL) test facility at SINAP. The performance is optimized through theoretical analysis and benchmarked with 4D Monte-Carlo simulations. The peak brightness of the source is expected to be larger than $2 * 10^{22}$ photons $/\left(\mathrm{mm}^{2} \cdot \operatorname{mrad}^{2} \cdot \mathrm{s} \bullet 0.1 \% \mathrm{BW}\right)$ at photon energy of $16.7 \mathrm{MeV}$. Its performance, compared to Extreme Light Infrastructure-Nuclear Physics (ELI-NP), and the SLEGS, is presented. The potential for basic and applied researches will also briefly outlined.

1. Q.Y. Pan et al., "A future laser Compton scattering (LCS) $\gamma$-ray source: SLEGS at the SSRF," Syn. Rad. News, 22(3), 11-20 (2009).

2. H.L. Wu, J.H. Chen, B. Liu, D. Wang, Z.T. Zhao, "Proposal for a high brightness $\gamma$-ray source at the SXFEL," Nuclear Science and Techniques, 26, 050103 (2015). 


\title{
Photons for Food and Medicine
}

\author{
C. Rangacharyulu \\ Department of Physics and Engineering Physics \\ University of Saskatchewan, \\ Saskatoon, SK, Canada, S7N 5E2 \\ Chary.r@usask.ca
}

Food is the most essential ingredient and Health is the indispensable factor to sustain life on this planet. Global food security is topical issue around the world with several research activities to increase the food production to meet the anticipated increase in demand in the coming decades. Even today, food supply does not feed the entire global population. About $30 \%$ of produce is unusable as it has short shelf life and/or becomes toxic due to pathogens. At present, food irradiation is not widely used nor its practice optimized to enhance the efficiency and minimize undesirable radiation effects. The characteristic attenuation factor vs photon energy dependence suggest that photons of a few hundred $\mathrm{keV}$ are better suited for soft vegetable produce while a few $\mathrm{MeV}$ energies are more effective for meat products etc. Further research with photon beams of $\mathrm{keV}-\mathrm{MeV}$ energies is warranted.

On medical front, production of medical isotopes either by photodisintegration process or photo fission mechanism is of interest. Significantly, the major components of fragment distribution are found to be nearly independent of the fissioning nucleus in neutron induced processes [1]. This suggests the possibility that photo fission fragment distributions may offer a good source of medical isotopes.

Furthermore, ion beam radiation therapy is too expensive and is not accessible in many countries. In view of the recent progress to achieve multi $\mathrm{GeV}$ acceleration gradients in a few centimeter laser wake field, it is tempting to consider if this technology can be adopted to generate a few hundred $\mathrm{MeV}$ per nucleon ion beams in a compact laser wake field setting. We are in the very early stages of simulations of this problem.

My talk will address these topics to stimulate discussions and hopefully research by others.

[1] R. Vogt and J. Randrup, Nuclear fission, in 100 years of Subatomic Physics, edited by Ernest Henley and Stephen Ellis, Pub: World Scientific (2013) 


\title{
Inverse-Compton Source of Twisted Photons
}

\author{
A. Afanasev \\ George Washington University, Washington, DC, USA \\ afanas@gwu.edu
}

Light beams with large values of the angular momentum along the direction of propagation is an active field of study in optics; a summary of their applications will be presented in this talk. These light beams are known as optical vortices or, when quantized, as twisted photons. Extension of this concept to the beams of neutrons and electrons was recently demonstrated experimentally.

We discuss a possibility of generating twisted photons at high energies via Compton backscattering, making them relevant for nuclear and high-energy physics. One of the challenges to be addressed is diagnostics of the generated twisted photons. We will review basic theory of the twisted photons and demonstrate that in their interactions with elementary quantum systems, like a hydrogen atom, the photo-absorption process follows new quantum selection rules for high-multipole excitations. Circular dichroism for the twisted photons interacting with isotropic targets is an observable that is zero for plane-wave photons due to parity conservation, therefore it can be useful for twisted-photon polarimetry. 


\title{
Mono-energetic x/gamma-ray sources based on ICS and their applications
}

\author{
Chuanxiang Tang ${ }^{1}$ \\ ${ }^{1}$ Department of Engineering Physics, Tsinghua University, Beijing 100084, China \\ Tang.xuh@tsinghua.edu.cn
}

The X-ray has been one of the most important tools for scientific explorations and other applications, since it was discovered by Wilhelm Conrad Röntgen in 1895. To reduce the dose on the target or the background noise, mono-energetic x-ray sources are strongly demanded. Inverse Compton scattering (ICS) or Thomson scattering (TS) between relativistic electron beam and high power laser pulse, can generate mono-energetic high brightness x-ray light. And it is an effective way to produce gamma photon beams whose energy exceeds the capability of normal synchrotron radiation sources and free electron lasers.

Tsinghua University has been studying the TS/ICS x-ray sources since 2001. The first TS experiment was done with a $16 \mathrm{MeV}$ BWT linac and a 1.5J, 6ns Q-switched Nd:YAG laser, and flux of $1.7 \times 10^{4}$ photons /pulse was measured with an MCP [1]. Tsinghua Thomson Scattering Xray Source (TTX) was designed with an s-band photocathode rf gun and a 3-meter SLAC TW accelerating structure [2,3], and its first light was got in 2011, with photon energy of 50keV, and flux of $10^{6}$ photons/pulse of $10 \mathrm{~Hz}$ [4,5]. Upgrading of TTX has been finished and the photon flux of $2 \times 10^{7} /$ pulse with photo energy of $50 \mathrm{keV}$ and flux stability of about $3 \%$ achieved this year. Phase contrast imaging and other applications with TTX have been carried out [6].

And another gamma source based on inverse Compton scattering as a user facility (XGLS) is under design, and will be built in the following years.

1. Y.C. Du, et al, "Preliminary experiment of the Thomson scattering X-ray source at Tsinghua University", CPC, 1, 32(2008).

2. C.X. Tang, et al., "Tsinghua Thomson scattering X-ray source", NIM A, 1,608(2009)

3. C.X. Tang, et al., "A simulation study of Tsinghua Thomson scattering X-ray source", CPC, S2, 33 ( 2009).

4. Y.C.Du, et al, "Soft X-ray generation experiment at the Tsinghua Thomson scattering X-ray source", NIM A, S1, 637(2011).

5. Y.C. Du, et al., "Generation of first hard X-ray pulse at Tsinghua Thomson Scattering X-ray Source", RSI, 5, 84(2013).

6. Z. Zhang, et al., "In-line phase-contrast imaging based on Tsinghua Thomson scattering xray source", REVIEW OF SCIENTIFIC INSTRUMENTS , 8, 85(2014). 


\section{Page Intentionally Left Blank}

Proc. of SPIE Vol. 10419 1041901-72 Downloaded From: https://www.spiedigitallibrary.org/conference-proceedings-of-spie on 26 Apr 2023
Terms of Use: https://www.spiedigitallibrary.org/terms-of-use 


\section{Tuesday, October 18, 2016 Advanced Optics \& Accelerator Technology: 16:50 18:45 \\ Session Chair: I. Jovanovic}




\title{
Perspectives on Ultra-Compact High Gradient RF Accelerator Technology
}

\author{
Sami G. Tantawi \\ SLAC National Accelerator Laboratory, Menlo Park, CA, USA \\ tantawi@slac.stanford.edu
}

Accelerating particles over shorter distances than ever before opens new doors in many areas of science. To this end, SLAC has been a leader in exploring RF breakdown phenomena in high vacuum structures. We have been able to engineer some of the materials used in the accelerator structure and modify its geometry to achieve extremely high gradients $\sim 175 \mathrm{MV} / \mathrm{m}$. Now, our research effort have to also include practical engineering developments to transform these advances into practical devices that can be applied to photon science, high-energy physics, medical, industrial and national security uses. For photon sciences, we are looking at compact high repetition rate coherent X-ray sources. In this talk We will describe our progress on high gradient RF accelerators and the equally advanced developments for compact RF sources capable of driving these new types of accelerators. 


\title{
20 years of X-ray Refractive Optics - Perspectives for MeV Light Sources.
}

\author{
Anatoly Snigirev \\ Immanuel Kant Baltic Federal University, 238300 Kaliningrad, Russia \\ anatyoly.snigirev@gmail.com
}

After the first successful experimental demonstration 20 years ago [1], the use of X-ray refractive optics has rapidly expanded and they are now in common use at 15 synchrotrons in 10 countries. This development has intensified after the successful implementation of transfocators tunable devices based on refractive lenses [2]. In addition to traditional micro-focusing applications, the transfocators can provide the following beam conditioning functions in the energy range from 3 to $200 \mathrm{keV}$ : condensers with a tunable beam size, micro-radian collimators, lowband pass filters - monochromators [2], high harmonics rejecters [3], Fourier transformers [4].

New advanced parameters of the beam provided by the diffraction-limited sources - XFELs and new synchrotrons with the reduced horizontal emittance will open up a unique opportunity to build up a new concept for the loss-free beam transport and conditioning systems based on in-line refractive optics. Taking an advantage of the substantially reduced horizontal source size and the beam divergence, these new systems integrated into the front-end can transfer the photon beam almost without losses from the front-end to any further secondary optical systems (mirrors, crystals, lenses etc.) or directly to the end-stations. Evidently, beamlines will benefit from the possibility to include the active moveable lens systems in the front-ends. In this regard, development of diamond refractive optics is crucial [5-7]. The implementation of the lens-based beam transport concept will significantly simplify the layout of majority of the new beamlines [8], opening new opportunities for the material science research under extreme conditions $[9,10]$. It will also allow a smooth beamlines transition from the present beam parameters to the upgraded ones, avoiding major optics modifications [11].

The field of applications of refractive optics is not limited to beam conditioning, but can be extended into the area of Fourier optics, as well as coherent diffraction and imaging techniques [12-15]. Using the intrinsic property of the refractive lens as a Fourier transformer, the coherent diffraction microscopy and high-resolution diffraction methods have been proposed to study $3-\mathrm{D}$ structures of photonic crystals and mesoscopic materials [16-18].

Another promising direction of refractive optics development is in-line X-ray interferometry. Recently proposed bi- and multi-lens interferometers can generate an interference field with a variable period ranging from tens of nanometers to tens of micrometers [19, 20]. This simple way to create an X-ray standing wave in paraxial geometry opens up the opportunity to develop new X-ray interferometry techniques to study natural and advanced man-made nano-scale materials, such as self-organized bio-systems, photonic and colloidal crystals, and nano-electronics materials. As a classical interferometer, it can be used for phase contrast imaging and radiography. Finally, it can be useful for the coherence characterization of the high energy X-rays sources [21]. 
1. A. Snigirev, V. Kohn, I. Snigireva, B. Lengeler, “A compound refractive lens for focusing high energy X-rays", Nature, 384, 49 (1996).

2. G.B.M. Vaughan, J.P. Wright, A. Bytchkov et al, "X-ray transfocators: focusing devices based on compound refractive lenses" J. Synchrotron Rad., 18, 125 (2011).

3. M. Polikarpov, I. Snigireva, A. Snigirev, "X-ray harmonics rejection on third-generation synchrotron sources using compound refractive lenses" J. Synchrotron Rad., 21, 484 (2014).

4. M. Lyubomirskiy, I. Snigireva, A. Snigirev, "Lens coupled tunable Young's double pinhole system for hard X-ray spatial coherence characterization” Optics express, 24, 13679 (2016).

5. M. Polikarpov, I. Snigireva, J. Morse et al, "Large - acceptance diamond planar refractive lenses manufactured by laser cutting” J. Synchrotron Rad., 22, 23 (2015).

6. S. Terentyev, V. Blank, S. Polyakov et al, "Parabolic single crystal diamond lenses for coherent X-ray imaging" Appl. Phys. Let., 107, 111108 (2015).

7. M. Polikarpov, I. Snigireva, A. Snigirev, "Focusing of white synchrotron radiation using largeacceptance cylindrical refractive lenses made of single - crystal diamond", AIP Conference Proceedings, 1741, 040024, 2016.

8. M. W. Bowler, D. Nurizzo, R. Barrett et al, "MASSIF-1: a beamline dedicated to the fully automatic characterization and data collection from crystals of biological macromolecules", J. Synchrotron Rad., 22, 1540 (2015).

9. N. Dubrovinskaia , L. Dubrovinsky, N. A. Solopova, et al, "Terapascal Static Pressure Generation with Ultrahigh Yield Strength Nanodiamond”, Sci. Adv., 2, e1600341, (2016).

10. F. Wilhelm, G. Garbarino, J. Jacobs, et al, "High pressure XANES and XMCD in the tender X-ray energy range", High Pressure Research, doi: 10.1080/08957959, 1206092 (2016).

11. Orange Book “ESRF Upgrade programme Phase II 92015-2022), Technical Design Study”, G. Admans, P. Berkvens, A. Kaprolat, J.L. Revol, eds., (2014).

12. V. Kohn, I. Snigireva, A. Snigirev, "Diffraction theory of imaging with compound refractive lens", Opt. Comm., 216, 247 (2003).

13. M. Drakopoulos, A. Snigirev, I. Snigirev, J. Schilling, "X-ray high resolution diffraction using refractive lenses", Appl. Phys. Lett., 86, 014102 (2005).

14. P. Ershov, S. Kuznetsov, I. Snigireva et al, "X-ray refractive optics as a Fourier transformer for high resolution diffraction", Appl. Cryst., 46, 1475 (2013).

15. H. Simons, A. King, W. Ludwig et al, "Dark-field X-ray microscopy for multiscale structural characterization”, Nature Communications, 6, 6098 (2015).

16. A. Bosak, I. Snigireva, K. Napolskii, A. Snigirev, "High energy transmission X-ray microscopy: a new tool for mesoscopic materials", Adv. Mater., 22, 3256 (2010).

17. D. V. Byelov, J.-M. Meijer, I. Snigireva et al, "In-situ hard x-ray microscopy of self-assembly in colloidal suspensions", RSC Advances, 3, 15670.V (2013).

18. Kohn, I. Snigireva, A. Snigirev, "Propagation of an X-ray beam modified by a photonic crystal", J. Synchrotron Rad., 21, 729 (2014).

19. A. Snigirev, I. Snigireva, V. Kohn et al, "X-ray nanointerferometer based on Si refractive bilenses", Phys. Rev. Lett., 103, 064801 (2009).

20. A. Snigirev, I. Snigireva, M. Lyubomirskiy, et al, "X-ray multilens interferometer based on Si refractive lenses", Optics express, 22(21), 25842 (2014).

21. M. Lyubomirskiy, I. Snigireva, V. Kohn, et al, "30-lens interferometer for high energy Xrays”, J. Synchrotron Rad., 23, doi:10.1107/S160057751601153X (2016). 


\title{
Introduction to Refractive Hard X-ray Optics and Perspectives for Operation at MeV Energies
}

\author{
Frank Seiboth ${ }^{1,2}$ \\ ${ }^{1}$ Deutsches Elektronen-Synchrotron DESY, Notkestr. 85, 22607 Hamburg, \\ Germany \\ ${ }^{2}$ Linac Coherent Light Source, SLAC National Accelerator Laboratory, \\ 2575 Sand Hill Road, 94025 Menlo Park, CA, USA
}

When X-rays were first discovered by W. C. Röntgen in 1895 he was not able to observe refraction of this new type of light with the given methods at that time [1]. Even almost a century later the feasibility of refractive X-ray optics was a controversy [2]. It was not until 1995 when Snigirev et al. demonstrated refractive focusing of hard X-rays using cylindrical lenses [3]. From this point on these lenses have seen an increasing popularity and have been refined countless times. Nowadays they are an important optical element at almost any hard X-ray synchrotron radiation source.

In this talk I will give a basic introduction to the refraction of light in the hard X-ray regime. The concept and basic parameters of compound refractive X-ray lenses will be shown together with recent applications in the hard X-ray regime. Finally, I will show perspective for the use of refractive lenses towards an operation at $\mathrm{MeV}$ energies.

1. W.C. Röntgen, "Über eine neue Art von Strahlen", Sitzungsberichte der Würzburger Physik.-Medic.-Gesellschaft, (1895).

2. A.G. Michette, "No X-ray lens", Nature 353, 6344 (1991).

3. A. Snigirev et al., "A compound refractive lens for focusing high-energy X-rays", Nature 384, 6604 (1996). 


\title{
High precision refractive index measurements of several materials at $\gamma$-ray energies up to above $1 \mathrm{MeV}$ - Towards $\gamma$ refractive optics
}

\author{
Marc M. Günther ${ }^{1,2}$ \\ ${ }^{1}$ Helmholtz Institute Jena, Jena, Thuringia, Germany \\ ${ }^{2}$ GSI-Helmholtz Center for Ion Research, Darmstadt, Hesse, Germany \\ m.guenther@gsi.de
}

The knowledge of the index of refraction is of crucial importance to understand basic physical phenomena like forward scattering processes. The refractive index is related to the forward scattering amplitude. Therefore, a direct approach occurs to the scattering process by measuring the index of refraction, enabling in such a way the investigation of scattering processes related to the frequency of the incident radiation and the properties of the irradiated material. In the X-ray regime, the refractive index measurements were performed with the main motivation to investigate the forward atomic scattering amplitude, which describes scattering of radiation incident on an atom [1]. Later, the knowledge of the X-ray refractive index was important to realize first refractive optics, which are well established in up-to-date X-ray imaging applications of biomedical as well as material sciences $[2,3]$. The energy dependent index of refraction is written as a complex number $n(E)=1+\delta(E)+i \beta(E)$. The real part $\delta$ describes the phase shift of the electro-magnetic wave after propagation through matter, and the imaginary part $\beta$ describes absorption processes. In the $\mathrm{X}$-ray regime, the decrement $\delta$ from $n=1$ is tiny $\left(10^{-5}-10^{-7}\right)$ and has a negative sign, means the refractive index is $n<1$. Within the $\gamma$ energy range quantum electro dynamic (QED) effects become relevance which influence the refractive behavior of matter. The knowledge of the $\gamma$ refractive index has a basic interest from an applied science point of view since it allows to design dedicated beam optics. The announced appearance of highly brilliant tunable $\gamma$-ray sources [4] motivates studies of the index of refraction at higher energies. Such sources will provide very intense photon pulses with tunable energies in the range of $0.1-20 \mathrm{MeV}$ and a typical bandwidth of $\Delta E / E \sim 10^{-3}$. One important application for these sources is to be found in the field of nuclear resonance fluorescence (NRF) experiments. Typical resonance energies are in the range of $100 \mathrm{keV}$ up to 8 $\mathrm{MeV}$, where the expected Doppler broadened resonance width is about $1 \mathrm{eV}$. Therefore, NRFresonance experiments one would require a very high degree of monochromatization. Via crystal diffraction the source bandwidth can be tuned to NRF-resonance requirements. But, the use of conventional crystal monochromators leads to losses because finite beam divergence of the source. An additional use of refractive optics makes this process sufficiently efficient to decrease the beam divergence compared to what is provided from the source.

In a recent experimental campaign for the first time we have measured the $\gamma$ refractive index of several solid as well as fluid materials with different atomic charge numbers $Z$ (between 10 and 80). The measured $\gamma$-ray energy range was $0.1-2 \mathrm{MeV}$. The experiment was performed at the new high-resolution $\gamma$ spectrometer GAMS6 at the Institut Laue-Langevin (ILL) in Grenoble (France). Currently, the refractive index of some materials was experimental determined up to $133 \mathrm{keV}$ photon energy, only [5]. At $\gamma$ energies the refractive index is very difficult to measure, because the refraction decreases strongly with energy. Sophisticated instrumentation is necessary, allowing high-resolution and high precision measurements of the refractive index. In the past, a first experiment was performed at the older spectrometer GAMS5 to measure the refractive index of Silicon. The result was an unexpected sign change of $\delta$ above $700 \mathrm{keV}$ and a first attempt in [6] to 
attribute it to virtual pair creation like Delbrück scattering turned out to be inadequate to explain the experimental findings. Theoretical work indicated the contribution from Delbrück scattering to $\delta$ is many orders of magnitude too low to account for the observed effect [7].

In this presentation, I will describe a new experimental methodology to measure the refractive index using the new ultra-high resolution $\gamma$ spectrometer GAMS6 and introduce the new results. Unknown potential systematic errors were found and removed, occurred at GAMS5 measurements. The new findings of the $\gamma$ refractive index of several materials provide a basis for the realization of $\gamma$ refractive optics. Based on the experimental data a first design study was performed for the construction of a $\gamma$ refractive lens system.

1. M. Deutsch et al., "High-accuracy structure-factor measurements in germanium", Phys. Rev. B, 42, 1248 (1990).

2. A. Snigirev et al., "A compound refractive lens for focusing high-energy X-rays", Nature, 384, 49 (1996).

3. B. Lengeler et al., "Refractive X-ray lenses", J. Phys. D: Applied Physics, 38, A218 (2005).

4. http://www.eli-np.ro and https://lasers.llnl.gov/science/photon-science/mega-ray.

5. M. Ruiz-Yaniz et al., "X-ray-refractive-index measurements at photon energies above $100 \mathrm{keV}$ with a grating interferometer", Phys. Rev. A, 91, 033803 (2015).

6. D. Habs et al., "Refractive Index of Silicon at $\gamma$ Ray Energies", Phys. Rev. Lett., 108, 184802 (2012).

7. J.T. Donohue, "Comment on "Refractive Index of Silicon at g Ray Energies"”, Phys. Rev. Lett., 110, 129501 (2013). 


\title{
Monochromatization of Gamma Rays to the eV level
}

\author{
Michael Jentschel \\ Institut Laue-Langevin, 71 Avenue des Martyrs, 38000 Grenoble, France
}

The wave properties of Gamma Rays allow a monochromatisation based on Laue-diffraction on perfect crystals. The talk summarizes the main physical principles, diffraction geometries and their according performances as well as the technological challenges of this approach. It will be demonstrated that a monochromatisation to the $\mathrm{eV}$ level is possible. We present first results from our new double crystal spectrometer GAMS6, installed at the ILL neutron high flux reactor and used for $\mathrm{eV}$ resolution spectroscopy of $(\mathrm{n}, \mathrm{g})$-reactions.

The possibility to easily tune bandwidth and energy via crystal parameters is of particular interest in the context of NRF-based material detection and assay. We will demonstrate the impact of crystal monochromatisation on a NRF-based detection experiment of $7 \mathrm{Li}$ at the European Synchrotron Radiation Facility. 


\section{Tuesday, October 18, 2016 \\ Evening Poster Session and Networking: 19:00 22:00 \\ Poster Abstracts can be found starting on page 153}




\section{Page Intentionally Left Blank}

Proc. of SPIE Vol. 10419 1041901-82 Downloaded From: https://www.spiedigitallibrary.org/conference-proceedings-of-spie on 26 Apr 2023
Terms of Use: https://www.spiedigitallibrary.org/terms-of-use 


\section{Wednesday, October 19, 2016 Nuclear Structure \& Astrophysics: 8:30 10:35 \\ Session Chair: N. Pietralla}




\title{
Photonuclear Research at HI $\gamma \mathrm{S}$ at Low Energies *
}

\author{
Calvin R. Howell \\ Duke University and the Triangle Universities Nuclear Laboratory \\ Durham, NC 27708 USA \\ howell@tunl.duke.edu
}

The High Intensity Gamma-ray Source $(\mathrm{HI} \gamma \mathrm{S})$ at the Triangle Universities Nuclear Laboratory (TUNL) provides beams that enable research on a variety of topics in basic and applied nuclear physics. During the last five years groups from about 40 institutions from around the world conducted research at $\mathrm{HI} \gamma \mathrm{S}$. The $\gamma$-ray beam at HI $\gamma \mathrm{S}$ is produced by Compton back-scattering of electrons from photons inside the optical cavity of a storage-ring based free electron laser. Circularly and linearly polarized $\gamma$-ray beams are available with beam polarization greater than 95\%. Beams are delivered to experiments with energies from 1 to $100 \mathrm{MeV}$ with energy spread selectable down to about $1 \%$ by collimation.

Nuclear physics research at $\mathrm{HI} \gamma \mathrm{S}$ can be described broadly in terms of phenomena in two energy regions, low and medium. The low-energy program includes studies of nuclear structure, nuclear astrophysics, few-nucleon reaction dynamics, fission and applications. Experiments in this part of the program are generally carried out at $\gamma$-ray beam energies below about $30 \mathrm{MeV}$, i.e., below the excitation region of the giant isovector dipole resonance. The main goal of the applied research is to measure quantities that are important to the development of technologies for applications in national nuclear and homeland security. Also, there is a small effort in nuclear medicine research at $\mathrm{HI} \gamma \mathrm{S}$. The other major effort at $\mathrm{HI} \gamma \mathrm{S}$ is the mediumenergy program. The primary thrust of this program is to perform measurements that contribute to bridging the conceptual and formulism gap in applying quantum chromodynamics (QCD) theory to describe nuclei and nuclear phenomena. The experiments in this area probe nuclear matter in the perturbative QCD regime where nucleons and mesons are the dominant effective degrees of freedom. This program component includes measurements of the nucleon electromagnetic and spin polarizabilities, evaluation of spin-dependent sum rules and investigation of symmetry breaking in the strong nuclear interaction.

In this talk, I will present an overview of the low-energy part of the research program at $\mathrm{HI} \gamma \mathrm{S}$. Another speaker at this conference will describe the medium-energy program. Recent results, current activities and plans for new experiments will be summarized.

\footnotetext{
*This work is supported in part by the U.S. Department of Energy Office of Nuclear Physics under grant no. DEFG02-97ER41033, the U.S. Department of Energy National Nuclear Security Administration Stewardship Science Academic Programs under grant no. DE-NA0002793, and the U.S. Department of Homeland Security Domestic Nuclear Detection Office under grant nos. DHS-2012-DN-077-ARI062 and 2010-DN-077-ARI46.
} 


\title{
Decay properties of the Pygmy Dipole Resonance
}

\author{
D. Savran \\ GSI Helmholtzzentrum für Schwerionenphysik, Germany \\ d.savran@gsi.de
}

Beside the Giant Dipole Resonance (GDR), many nuclei show the feature of additional low-lying electric dipole (E1) strength below and around the particle separation energies, which is usually denoted as Pygmy Dipole Resonance (PDR) [1]. The existence of the PDR in nearly every studied nucleus and the smooth variation of its properties lead to the assumption that the PDR is a newly discovered collective mode. While some of the gross characteristics are reproduced by different theoretical model descriptions, its detailed structure and the degree of collectivity are a matter of ongoing discussions.

An excellent tool to investigate bound E1 excitations is the method of nuclear resonance fluorescence (NRF) [2], which has been used in the last years to perform systematic studies of E1 strength below the neutron separation energy in nuclei of different mass regions [1]. Besides the possibility to perform systematic studies of the gross features of low-lying E1 strength this experimental method allows the investigation of the fine structure or of individual states using high-purity Germanium (HPGe) detectors in the $\gamma$-ray spectroscopy. Modern photon sources for this kind of experiments are bremsstrahlung and laser-Compton-backscattering (LCB). While experiments with bremsstrahlung allow to investigate a large energy region within one experimental run and to identify single photo-excited states, the mono-energetic and highly polarized character of LCB is ideal to investigate certain energy regions or individual states in detail. In addition, experiments with mono-energetic photons provide the possibility to get insight into the decay properties of the PDR [3]. To further increase the sensitivity to certain decay channels and to investigate in detail the decay behavior of the PDR we recently performed $\gamma-\gamma$ coincidence spectroscopy in combination with the LCB beam at the High-Intensity Photon Source (HI $\gamma \mathrm{S})$ using the new installed $\gamma^{3}$ setup [4]. An overview on the available experimental data on low-lying E1 strength obtained with the NRF method will be presented with a focus on experiments using LCB photons.

1. D. Savran, T. Aumann, A. Zilges, "Experimental Studies of the Pygmy Dipole Resonance", Prog. Part. Nucl. Phys., 70, 210 (2013).

2. U. Kneissl, H.H. Pitz, A. Zilges, "Investigation of Nuclear Structure by Resonance Fluorescence Scattering”, Prog. Part. Nucl. Phys., 37, 349 (1996).

3. B. Löher et al., "The decay pattern of the Pygmy Dipole Resonance of ${ }^{140} \mathrm{Ce}$, , Phys. Lett. B 756 (2016) 72

4. B. Löher et al., "The high-efficiency $\gamma$-ray spectroscopy setup $\gamma^{3}$ at HI $\gamma \mathrm{S}$ ", Nucl. Inst. and Meth. 723 (2013) 136 


\section{Photonuclear Precision Measurements on Nuclear Quadrupole Collectivity}

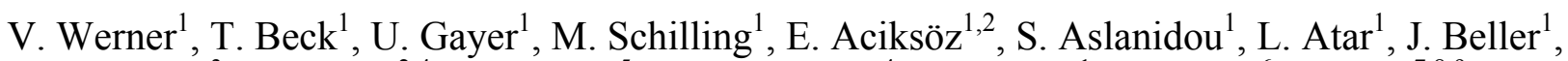
M. Bhike ${ }^{3}$, A. Coban ${ }^{2,4}$, N. Cooper ${ }^{5}$, D. Choudhury ${ }^{4}$, M. Cordts ${ }^{1}$, V. Derya ${ }^{6}$, J. Isaak ${ }^{7,8,9}$, J. Kleemann ${ }^{1}$, P. Koseoglou ${ }^{1}$, C. Kremer ${ }^{1}$, Krishichayan ${ }^{3}$, B.Löher ${ }^{9}$, L. Mertes ${ }^{1}$, H. Pai ${ }^{1}$, N.

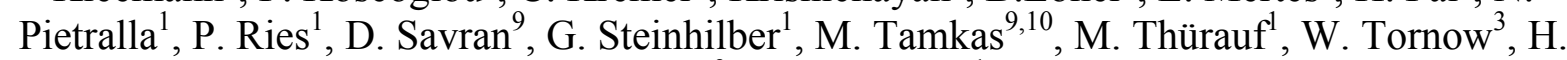
Weller $^{3}$, J. Wiederholt ${ }^{1}$

${ }^{I}$ Institut für Kernphysik, TU Darmstadt, Darmstadt, Germany, vw@ikp.tu-darmstadt.de

${ }^{2}$ Department of Physics, Akdeniz University, Antalya, Turkey

${ }^{3}$ Department of Physics, Duke University, Durham, NC, USA

${ }^{4}$ Extreme Light Infrastructure - Nuclear Physics, Bucharest-Magurele, Romania

${ }^{5}$ Department of Physics, University of Richmond, Richmond, VA, USA

${ }^{6}$ Institut für Kernphysik, Universität zu Köln, Köln, Germany

${ }^{7}$ ExtreMe Matter Institute, GSI, Darmstadt, Germany

${ }^{8}$ Frankfurt Institute for Advanced Studies, Frankfurt, Germany

${ }^{9}$ GSI Helmholtzzentrum für Schwerionenforschung, Darmstadt, Germany

${ }^{10}$ Istanbul Arel University, Mathematics and Computer Department, Istanbul, Turkey

The inelastic scattering of real photons off atomic nuclei, i.e., the method of nuclear resonance fluorescence (NRF), is a unique technique in the study of nuclear structure. This is mainly due to the low momentum carried by the photon, predominantly exciting electric and magnetic dipole modes. With today's shift from classical, that is usually bremsstrahlung, photon production to intense and brilliant near-monoenergetic photon sources through Comptonbackscattering, new levels of sensitivity can be reached and new observables become accessible, aided by the advent of efficient large gamma-ray spectrometers like $\gamma^{3}$ at HIGS [1], or the future ELIADE array at ELI-NP.

Examples are found in low-lying dipole, but also quadrupole excitations, which have been focus of present research at the S-DALINAC facility of TU Darmstadt and the HIGS facility at TUNL. Some experimental advances at both facilities will be discussed, which lay part of the ground work for future research at facilities like ELI-NP or an upgraded HIGS.

At low energies, the nuclear scissors mode [2-4], a counter oscillation of quadrupoledeformed proton and neutron bodies, is known to dominate the magnetic dipole-excitation strength. Making use of the high-intensity and fully-polarized gamma-ray beams at HIGS, we were able to perform novel studies of the scissors mode. Although this M1 mode has been focus of systematic studies for years and much information on excitation energies and probabilities have been collected [5], data on its detailed decay schemes or on collective structures built upon the scissors mode is sparse or not available at all.

Other present studies focus on isotopes which are candidates for the mother or daughter nuclei of neutrino-less double-beta $(0 v \beta \beta)$ decay. Should this rare decay mode exist, i.e., if the neutrino is a Majorana particle, nuclear models will be used to extract a neutrino mode from observed $0 v \beta \beta$ transition rates. It has been shown for ${ }^{154} \mathrm{Gd}[6]$ that the energy and absolute decay probabilities to states other than the ground state, in particular the first excited $0^{+}$state in nuclei with even numbers of protons and neutrons, are sensitive to isovector parameters in nuclear models, such as the proton-neutron interacting boson model (IBM-2) [3]. Many 0v $\beta \beta$ 
candidates, hence, baseline isotopes for $0 v \beta \beta$ detectors, are in the vicinity of structural change on the transition (sometimes phase transition) from spherical to deformed nuclei. The decay paths of the scissors mode are especially sensitive to such transitional properties and can further constrain nuclear models. Also in ${ }^{154} \mathrm{Gd}$, we showcase for the first time the possibility to observe an excited state of the rotational scissors band using high-resolution spectroscopy in combination with the highly-intense HIGS beams.

New data has been obtained on ${ }^{112} \mathrm{Sn}$ at the S-DALINAC, with consequences on an ongoing discussion on $\mathrm{B}(\mathrm{E} 2)$ excitation strengths in the $\mathrm{Sn}$ isotopic chain. Literature data from experiments using Doppler-shift techniques [7] and Coulomb excitation (e.g., Refs. [8,9]) are at variance leading to significant differences for the systematics in this important proton-magic isotopic chain, even for its stable members. These differences magnify in neutron-deficient isotopes toward the "holy grail" doubly-magic $\mathrm{N}=\mathrm{Z}$ nucleus ${ }^{100} \mathrm{Sn}$, since measurements typically rely on supposedly well-known stable-isotope data. Although the photo-excitation of quadrupole-excited states is less likely than that of dipole-excited states, it does occur and allows to measure the E2 strengths of the first excited $2^{+}$state. We applied this technique, which is independent from nuclear models or constraints of the Doppler-shift or Coulomb-excitation methods, for the first time to ${ }^{112} \mathrm{Sn}$, and compare to data from the other methods.

1. B. Löher et al., Nucl. Inst. Methods A 723, 136 (2013).

2. N. Lo Iudice and F. Palumbo, Phys. Rev. Lett. 41, 193 (1979).

3. F. Iachello and A. Arima, "The Interacting Boson Model" (Cambridge University Press, Cambridge, 1987).

4. D. Bohle et al., Phys. Lett. B 195, 326 (1987).

5. K. Heyde et al., Rev. Mod. Phys. 82, 2365 (2010).

6. J. Beller et al., Phys. Rev. Lett. 111, 172501 (2013).

7. A. Jungclaus et al., Phys. Lett. B 695, 110 (2011).

8. P. Doornenbal et al., Phys. Rev. C 90, 061302(R) (2014).

9. J. M. Allmond et al., Phys. Rev. C 92, 041303 (2015). 
Addressing Astrophysics Questions with laser-Compton Gamma-ray Sources*

\author{
Moshe Gai \\ LNS at Avery Point, University of Connecticut, Groton, CT 06340-6097, USA \\ Email: moshe.gai@uconn.edu, http://astro.uconn.edu
}

An extensive research program in Nuclear Astrophysics is currently occurring at the HI $\gamma \mathrm{S}$ facility [1,2,3] in the USA and is planned for the ELI-NP facility [4], mainly within the Charged Particle Working group (Gamma-TDR\#4) [5]. These research program with gamma-ray beams involve the detection of charged particles and neutrons emanating from the time reversed reaction: ( $\gamma$,particle), with a cross section that is enhanced with respect to the direct reaction by the kinematical enhancement of detailed balance. In addition measurement of cross section of importance in nuclear astrophysics are planned for the ELI-NP in the laser produced plasma as for example carried out at the U Texas Petawatt Laser facility [6] mimicking stellar conditions. We will review this rich research program that promises to solve some of the central problems in the field including the four decade quest to measure the carbon to oxygen ratio at the end of helium burning (by measuring the ${ }^{16} \mathrm{O}(\gamma, \alpha)$ reaction).

* This work is supported in part by the U.S. Department of Energy, Grants No. DE-FG0294ER40870 and by the NSF: US-Romania, Catalyzing New International Collaboration (NSF-CNIC) award number 1443249.

[1] Henry R. Weller, Mohammad W. Ahmed, Haiyan Gao, Werner Tornow, Ying K. Wu, Moshe Gai and Rory Miskimen, Prog. Part. Nucl. Phy. 62, 257 (2009).

[2] C. W. Arnold, T. B. Clegg, C. Iliadis, H. J. Karwowski, G. C. Rich, and J. R. Tompkins and C.R. Howell, Phys. Rev. C 85, 044605 (2012).

[3] M. Gai, M.W. Ahmed, I. Gheorghe, M. Freer, C.R. Howell, D.P. Kendellen, S.R. Stern, V. Zamfir, Contribution to Nuclear Photonics 2016, Monterey, CA, October 16-21, 2016.

[4] D. Filipescu, A. Anzalone, D.L. Balabanski, S.S. Belyshev, F. Camera, M. La Cognata, P. Constantin, L. Csige, P.V. Cuong, M. Cwiok, V. Derya, W. Dominik, M. Gai, S. Gales, I. Gheorghe, B.S. Ishkhanov, A. Krasznahorkay, A.A. Kuznetsov, C. Mazzocchi, V.N. Orlin, N. Pietralla, M. Sin, C. Spitaleri, K.A. Stopani, O. Tesileanu, C.A. Ur, I. Ursu, H. Utsunomiya, V.V. Varlamov, H.R. Weller, N.V. Zamfir, and A. Zilges,

Eur. Phys. J. A51, 185 (2015).

[5] O. Tesileanu, M. Gai, A. Anzalone, C. Balan, J.S. Bihalowicz, M. Cwiok, W. Dominik, S. Gales, D.G. Ghiță, Z. Janas, D.P. Kendellen, M. La Cognata, K. Mikszuta, C. Petcu, M. Pfützner, T. Matulewicz, C. Mazzocchi, C. Spitaleri;

Romanian Reports in Physics 68, S699 (2016).

[6] M. Barbui, W. Bang, A. Bonasera, K. Hagel, K. Schmidt, J.B. Natowitz, R. Burch, G. Giuliani, M. Barbarino, H. Zheng, G. Dyer, H.J. Quevedo, E. Gaul, A.C. Bernstein, M. Donovan, S. Kimura, M. Mazzocco, F. Consoli, R. De Angelis, P. Andreoli, T. Ditmire, Phys. Rev. Lett. 111, 055002 (2013). 


\title{
$s$-Process Nucleosynthesis Using Nuclear Resonance Fluorescence
}

\author{
Anton Tonchev \\ Lawrence Livermore National Laboratory, \\ P.O. Box 808, Livermore, CA 94551-0808, USA
}

Accurate neutron-capture cross sections for radioactive nuclei near the line of beta stability are crucial for understanding the $s$-process nucleosynthesis. However, neutron-capture cross sections for short-lived radionuclides are difficult to measure due to the fact that the measurements require both highly radioactive samples and intense neutron sources. Essential ingredients for describing the $\gamma$ decays following neutron capture are the $\gamma$-ray strength function and level densities. We will compare different indirect approaches for obtaining the most relevant observables that can constrain Hauser-Feshbach statistical model calculations of capture cross sections. Specifically, we will consider photon scattering using monoenergetic and 100\% linearly polarized photon beams $[1,2]$. Challenges that exist on the path to obtaining neutroncapture cross sections for reactions on isotopes near and far from stability will be discussed $[3,4]$.

* This work was performed under the auspices of US DOE by LLNL under contract DEAC52-07NA27344.

1. R. Raut et al. Phys. Rev. Lett. 111, 112501 (2013).

2. A. Sauerwein, et al. Phys. Rev. C 89, 035803 (2014).

3. A. Spyrou et al. Phys. Rev. Lett. 113, 232502 (2014).

4. J.E. Escher, et al. Conference Proceeding submitted to the 5th International Workshop on Compound-Nuclear Reactions and Related Topics, October 19-23, 2015, Tokyo Institute of Technology. 


\section{Page Intentionally Left Blank}

Proc. of SPIE Vol. 10419 1041901-90

Downloaded From: https://www.spiedigitallibrary.org/conference-proceedings-of-spie on 26 Apr 2023 Terms of Use: https://www.spiedigitallibrary.org/terms-of-use 


\section{Wednesday, October 19, 2016 \\ Compton Sources Based on Intense Lasers: 10:55 13:15 \\ Session Chair: C. Brenner}




\title{
Development and Applications of Laser-Wakefield-Accelerator-Driven Compton X-Rays
}

\author{
Donald Umstadter ${ }^{1}$ \\ ${ }^{1}$ University of Nebraska-Lincoln, Lincoln, NE, USA \\ donald.umstadter@unl.edu
}

A compact inverse Compton-scattering x-ray light source is discussed. It is driven by an ultra-high-gradient laser-wakefield electron accelerator $(>1 \mathrm{GeV} / \mathrm{cm})$, which is itself driven by multi-terawatt peak power laser system [1]. The x-rays generated have several unique characteristics: $10-\mathrm{Hz}$ repetition rate, low angular divergence $(5 \mathrm{mrad})$, narrow bandwidth $(\Delta E / E$ $\leq 25 \%$ ), and tunability over a large photon energy range (0.1-10 MeV) [2--4]. This is also the first all-laser-driven hard x-ray source with a peaked photon-number spectrum [3]. Also discussed are recent applications of this novel source for high spatial resolution and low radiological dose radiographic imaging of standard objects through thick steel shielding [5], photonuclear activation [6], as well as measurement of intrinsic laser-wakefield accelerated electron-beam emittance [7]. Recent results on the use of this source to study highly nonlinear scattering [8] and fundamental theories of high-field electrodynamics - such as radiationreaction [9]—will also be discussed.

[1] D. P. Umstadter, Contemporary Physics 56, 417 (2015).

[2] S. Chen, N. Powers, I. Ghebregziabher, C. Maharjan, C. Liu, G. Golovin, S. Banerjee, J. Zhang, N. Cunningham, and A. Moorti, Phys. Rev. Lett. 110, 155003 (2013).

[3] N. D. Powers, I. Ghebregziabher, G. Golovin, C. Liu, S. Chen, S. Banerjee, J. Zhang, and D. P. Umstadter, Nature Photonics 8, 28 (2014).

[4] C. Liu, G. Golovin, S. Chen, J. Zhang, B. Zhao, D. Haden, S. Banerjee, J. Silano, H. Karwowski, and D. Umstadter, Opt. Lett. 39, 4132 (2014).

[5] S. Chen, G. Golovin, C. Miller, D. Haden, S. Banerjee, P. Zhang, C. Liu, J. Zhang, B. Zhao, and S. Clarke, Nuclear Instruments and Methods in Physics Research Section B: Beam Interactions with Materials and Atoms 366, 217 (2016).

[6] S. Banerjee, G. Golovin, P. Zhang, D. Haden, S. Chen, C. Liu, J. Zhang, B. Zhao, K. Brown, and J. Mills, in SPIE Optics Optoelectronics (International Society for Optics and Photonics, 2015), p. 95151C.

[7] G. Golovin, S. Banerjee, C. Liu, et al, Sci. Rep. 6, 24622 (2016).

[8] Y. Lau, F. He, D. P. Umstadter, and R. Kowalczyk, Physics of Plasmas (1994-present) 10, 2155 (2003).

[9] D. A. Burton and A. Noble, Contemporary Physics 55, 110 (2014). 


\title{
Compact quasi-monoenergetic photon sources for nuclear applications using laser-plasma accelerators
}

Cameron G.R. Geddes ${ }^{1}$, Hai-En Tsai ${ }^{1}$, Jeroen van Tilborg ${ }^{1}$, Sam Barber ${ }^{1}$, Carlo Benedetti ${ }^{1}$, Fumika Isono $^{1}$, Remi Lehe ${ }^{1}$, Bernhard Ludewigt ${ }^{1}$, Hann-Shin Mao ${ }^{1}$, Kei Nakamura ${ }^{1}$, Brian J. Quiter $^{1}$, Carl B. Schroeder ${ }^{1}$, Sven Steinke ${ }^{1}$, Kelly Swanson ${ }^{1}$, Csaba Toth ${ }^{1}$, Jean-Luc Vay ${ }^{1}$, Kai

Vettter $^{1}$, Yigong Zhang ${ }^{1}$, David Grote ${ }^{2}$, Alex Friedman ${ }^{2}$, Eric Esarey ${ }^{1}$, Wim Leemans ${ }^{1,3}$

\author{
${ }^{1}$ Lawrence Berkeley National Laboratory, Berkeley CA, USA \\ ${ }^{2}$ Lawrence Livermore National Laboratory, Livermore CA, USA \\ ${ }^{3}$ also at: Department of Physics, U.C. Berkeley, Berkeley CA, USA \\ email:cgrgeddes@lbl.gov
}

Near-monoenergetic photon sources at $\mathrm{MeV}$ energies offer improved sensitivity at reduced dose for nuclear nonproliferation and related applications. Applications include cargo screening, active interrogation, treaty verification, nondestructive assay, and emergency response. Thomson (also referred to as Compton) scattering sources are an established method to produce appropriate photon beams. Applications are however restricted by the size of the required highenergy electron linac, scattering (photon production) system, and shielding for disposal of the high-energy electron beam. Laser-plasma accelerators (LPAs) produce GeV electron beams in centimeters, using the plasma wave driven by the radiation pressure of an intense laser. Recent LPA experiments demonstrated greatly improved beam quality and efficiency, rendering them strong candidates for compact high-quality photon sources. Designs for $\mathrm{MeV}$ photon sources utilizing the unique properties of LPAs are presented. Control of the scattering laser, including plasma guiding, can reduce scattering laser size and/or electron beam current requirements to scale compatible with the LPA. The plasma structure can decelerate the electron beam after photon production, reducing the size and mass of shielding required for beam disposal. Status of an experimental facility and of experiments to demonstrate and combine these techniques will be described, together with the path to a compact photon source system. 


\title{
Bright $X$ - and $\gamma$-ray sources based on a high-quality laser wakefield accelerator
}

\author{
J. S. Liu, W.T. Wang, C.H. Yu, R. Qi, W.T. Li, C. Wang, Z.J. Zhang, J.Q. Liu, Z.Y. Qin, M. \\ Fang, Y. Xu, Y.X. Leng, R.X.Li, Z.Z.Xu \\ State Key Laboratory of High Field Laser Physics, Shanghai Institute of Optics and Fine \\ Mechanics (SIOM), Chinese Academy of Sciences, Shanghai 201800, China \\ *michaeljs_liu@siom.ac.cn
}

In a laser wakefield accelerator (LWFA), a relativistic femtosecond laser pulse can drive a plasma-density wake with ultra-high accelerating fields reaching $100 \mathrm{GV} / \mathrm{m}$, in which trapped electrons can be accelerated to $\mathrm{GeV}$-class over short distance on a centimeter-scale. Remarkable progress has been made over the past decade in generating quasi-monoenergetic $\mathrm{GeV}$ electron beams (e-beams) [1-3], making the LWFA promising as a compact accelerator, which will have potential applications, such as X-ray free electron lasers, X-ray and $\gamma$-ray radiation sources.

In this work, we presented the latest experimental results of bright $\mathrm{X}$ - and $\gamma$-rays generation via two different schemes such as inverse Compton scattering, and betatron radiation based on a high-quality cascaded LWFA which was powered by a 5-Hz 200-TW femtosecond laser facility at SIOM. The cascaded LWFA developed at SIOM has the ability to generate tunable highquality e-beams $(<1 \%$ rms energy spread, $\sim 80 \mathrm{pC}$ at the peak energy tunable from 200 to 500 $\mathrm{MeV},<0.4 \mathrm{mrad} \mathrm{rms}$ divergence), which were used to generate compact femtosecond X-ray sources [4]. By employing a self-synchronized all-optical Compton scattering scheme, in which the electron beam collided with the intense driving laser pulse via the reflection of a plasma mirror, we produced tunable quasi-monochromatic $\mathrm{MeV} \gamma$-rays ( $33 \%$ full-width at halfmaximum) with a peak brilliance of $\sim 3.1 \times 10^{22}$ photons s${ }^{-1} \mathrm{~mm}^{-2} \mathrm{mrad}^{-2} 0.1 \% \mathrm{BW}$ at $1 \mathrm{MeV}$, which is one order of magnitude higher than ever reported value in $\mathrm{MeV}$ regime to the best of our knowledge [5]. By manipulating the plasma density distribution, we obtained very bright betatron radiation at several-tens $\mathrm{keV}$ controllable in yield and peak energy.

1. J. S. Liu, et al., "All-optical cascaded laser wakefield accelerator using ionization-induced injection”. Phys. Rev. Lett. 107, 035001 (2011).

2. X. Wang, et al., "Quasi-monoenergetic laser-plasma acceleration of electrons to $2 \mathrm{GeV}$ ". Nat. Commun. 4, 1988 (2013).

3. W. P. Leemans, et al., "Multi-GeV electron beams from capillary-discharge-guided subpetawatt laser pulses in the self-trapping regime". Phys. Rev. Lett. 113, 245002 (2014)

4. W. T. Wang et al., "High-brightness high-energy electron beams from a laser wakefield accelerator via energy chirp control". (to be published).

5. C. H. Yu et al., "Ultrahigh brilliance quasi-monochromatic $\mathrm{MeV} \gamma$-rays based on selfsynchronized all-optical Compton scattering”. Sci. Rep. 6, 29518 (2016). 


\section{Multi-GeV electron acceleration and PW Laser-driven Compton Gamma-rays}

Hyung Taek Kim ${ }^{1,2^{*}}$, V. B. Pathak ${ }^{1}$, Ki Hong Pae ${ }^{1,2}$,A. Lifschitz ${ }^{3}$, F. Sylla ${ }^{4}$, Jung Jun Shin ${ }^{1}$, C. Hojbotoa $^{1,5}$, Seong Ku Lee ${ }^{1,2}$, Jae Hee Sung ${ }^{1,2}$, Hwang Woon Lee ${ }^{1}$, E. Guillaume ${ }^{3}$, Kazuhisa Nakajima $^{1}, \mathrm{~V} . \mathrm{Malka}^{3}$, and Chang Hee $\mathrm{Nam}^{1,5}$

${ }^{1}$ Center for Relativistic Laser Science, IBS, Gwangju 61005, Korea ${ }^{2}$ Advanced Photonics Research Institute, GIST, Gwangju 61005, Korea

${ }^{3}$ Laboratoire d'Optique Appliquée (LOA), ENSTA, 91762 Palaiseau, France

${ }^{4}$ SourceLAB SAS, 86 rue de Paris, 91400 Orsay, France.

${ }^{5}$ Dept. of Physics and Photon Science, GIST, Gwangju 61005, Korea

\section{"htkim@gist.ac.kr}

The laser wakefield acceleration (LWFA) is one of the most attractive research areas in laserplasma research because of its strong potential for a next generation accelerator. In the regime of relativistic laser-plasma interactions, LWFA has paved the route to develop compact electron accelerators and radiation sources. With the recent progress of intense laser technology multi-PW lasers are realized that can provide chances to develop compact multi-GeV laser electron accelerators. We have developed two PW Ti:Sapphire laser beamlines [1], and successfully applied the PW laser pulses to generate a 3-GeV electron beam [2]. Recently, we also developed a new method to stabilize multi-GeV electron beams by controlling the waveform of PW laser pulses. Furthermore, we are in the process of upgrading our PW laser to a 20-fs, 4-PW laser. Here, we present the recent progress in LWFA research with PW laser pulses and the plans for developing 10-GeV electron beam and Compton gamma-ray sources driven by the 4-PW laser and for investigating QED effects in photon-electron interactions and nuclear photonics.

1. J. H. Sung, S. K. Lee, T. J. Yu, T. M. Jeong, and J. Lee, Opt. Lett. 35, 3021 (2010).

2. H. T. Kim, K. H. Pae, H. J. Cha, I J. Kim, T. J. Yu, J. H. Sung, S. K. Lee, T. M. Jeong, J. Lee, Phys. Rev. Lett. 111, 165002 (2013). 


\section{Physics and Technology of All-Optical Inverse Compton Scattering Sources}

Yusuke Sakai, Ivan Gadgev, Oliver Williams, Atsushi Fukasaw and James Rosenzweig

University of California at Los Angeles

High average flux inverse Compton scattering (ICS) sources based on linear accelerators have emerging advanced applications in medicine, scientific imaging, industry and defense. We review recent progress in their experimental development, at UCLA and beyond, based on all-optical methods, including acceleration via the inverse free-electron laser (IFEL) mechanism. We examine new physics and instrumentation results, and discuss the use of laser recirculation in both the ICS and IFEL systems. 


\title{
Staging of laser-plasma accelerators to further accelerate or decelerate electron beams for compact photon source applications
}

\author{
E. Esarey, S. Steinke, J. van Tilborg, C. Benedetti, C. G. R. Geddes, J. \\ Daniels, K. K. Swanson, A. J. Gonsalves, K. Nakamura, B. H. Shaw, C. B. \\ Schroeder, and W. P. Leemans \\ Lawrence Berkeley National Laboratory, Berkeley, California 94720, USA \\ ehesarey@lbl.gov
}

Laser-plasma accelerators (LPAs) are of interest to compact photon sources due to their ability to sustain ultrahigh accelerating gradients $(10-100 \mathrm{GV} / \mathrm{m})$, some three orders of magnitude beyond those of conventional accelerators. Staging of independently powered LPA modules is necessary to obtain high electron energies in a compact system. Recent LBNL experiments have demonstrated the staging of two modules that are coupled at a short distance by a plasma mirror [1]. Stable electron beams from the first stage were focused by an active plasma lens into the second stage. This permitted electron beam trapping and an additional energy gain of $100 \mathrm{MeV}$ in the second stage. By varying the arrival time of the electron beam relative to the drive laser in the second stage, the second stage can decelerate the beam. Simulations indicate that this can be a very effective method for decelerating the electron beam to very low energies, thus circumventing the need for a large, conventional, high-energy electron beam dump.

This work was supported by the U.S. Department of Energy, Office of High Energy Physics, and by the National Nuclear Security Administration, Defense Nuclear Nonproliferation R\&D.

1. S. Steinke et al., Nature 530, 190 (2016); S. Steinke et al., Phys. Plasmas 23, 056705 (2016). 


\section{Page Intentionally Left Blank}

Proc. of SPIE Vol. 10419 1041901-98

Downloaded From: https://www.spiedigitallibrary.org/conference-proceedings-of-spie on 26 Apr 2023 Terms of Use: https://www.spiedigitallibrary.org/terms-of-use 


\section{Thursday, October 20, 2016 \\ Photon-based Particles Sources: 8:30 10:50 \\ Session Chair: K. Tanaka}




\title{
Intense Ion, Neutron and X-rays Beams from relativistic Laser-matter interaction
}

\section{Can lasers complement or even replace particle accelerators for radiation facilities?}

\author{
Markus Roth \\ Institute for Nuclear Physics, Technische Universität Darmstadt, Germany
}

The quest for laser-based high-energy ions and secondary radiation for applications like material research or even cancer treatment has been going on for some years. Recently, using high contrast short pulse lasers like the TRIDENT in the US or PHELIX laser in Germany laser and the concept of relativistic transparency, a breakthrough has been achieved with ion energies exceeding $100 \mathrm{MeV}$ and the production of intense neutron pulses [1], only about three orders of magnitude weaker than the LANSCE neutron pulses.

Based on the new mechanism's advantages, a laser-driven deuteron beam is used to achieve a new record in laser-neutron production in intensity, energy and directionality. Thus, we demonstrated the use of short pulse lasers to use the resulting hard X-Rays and neutrons of different energies to radiograph an unknown object and to determine its material composition [2]. Neutron generation, scale exponentially with energy of the deuterium beam, which scales with the energy of the accelerating laser and result in a collimated beam, allowing e.g. a much higher fraction of produced neutrons to be captured by the moderator and delivered to the application. With available laser power increasing and the prospected increase in repetition rate and therefore average power, pulsed neutron sources achieving the neutron output of LANSCE or even SNS are conceivable. Since comparably little shielding is required, targets for laser neutron sources can be very compact, allowing moderator to sample/detector distances of a meter or even less, further increasing the flux on the sample. Investment and operational cost as well as real estate foot-print for the necessary laser systems are all a small fraction of those for the particle accelerators or reactors required for present neutron sources. We quantitatively compare the initial experiments in laser neutron generation with existing conventional sources. An overview and outlook on the developments in laser technology will be presented and the potential for neutron production will be outlined.

[1] M. Roth et al., Phys. Rev. Lett., 110, p. 044802 (2013)

[2] D. Jung et al., Physics of Plasmas 20, 056706 (2013)

[3] ELI-BEAMS, L4 Beamline

[4] DIPOLE Laser system, Rutherford Appleton Laboratory, UK

[5] J.M.Cole et al., NATURE Scientific RepoRts I 5:13244

Proc. of SPIE Vol. 10419 1041901-100 


\title{
Laser-driven quantum beams from ultra-intense laser-matter interactions
}

\author{
Tatsufumi Nakamura ${ }^{1}$, Takehito Hayakawa ${ }^{2}$ \\ ${ }^{1}$ Fukuoka Institute of Technology, Fukuoka, Fukuoka, Japan \\ t-nakamura@fit.ac.jp \\ ${ }^{2}$ National Institute for Quantum and Radiological Science and Technology, Tokai, Ibaraki, \\ Japan \\ hayakawa.takehito@qst.go.jp
}

An irradiation of ultra-intense laser beam on solid target leads to generations of various quantum beams of electrons, ions, and x-rays. In the higher intensity regime where a radiation reaction effect on an electron in the laser field plays an important role, the electron motion becomes dissipative and the laser energy is effectively converted into the radiation energy. When laser and plasma parameters are properly chosen, the radiation has a spectral peak in the $\gamma$-ray regime. This leads to a proposal of laser-driven $\gamma$-rays with unique features such as high intensity, well-collimation, and ultra-short duration $[1,2]$.

This also reveals that investigations of the energy transport by the laser-driven $\gamma$-rays become crucial for understanding the laser-plasma interactions. We then developed a Particle-inCell code by including transport processes of the laser-driven $\gamma$-rays in materials, such as Compton scattering, electron-positron pair creation, and photo-nuclear reactions [3]. By using the code, possibilities of laser-driven positron and neutron source using the $\gamma$-rays generated via the radiation reaction effect are investigated [4]. Positrons are generated by the transport of $\gamma$ rays inside of the target via Bethe-Heitler process. The generated positrons propagate through the target and accelerated by a sheath electric field induced at the target rear surface, which results in a generation of energetic and quasi-monochromatic positron beams. The positron beam is as well-collimated as the laser driven $\gamma$-rays, and as short as the incident laser pulse. A parametric study explores a suitable condition for an efficient generation of quasi-monochromatic positron beams. Neutrons are also generated via the photo-nuclear reaction using the laser-driven $\gamma$-rays. Since the difference of the threshold energy and photonuclear cross section, a choice of target material is important. The characteristics of these laser-driven quantum beams and suitable conditions for their generations will be discussed.

1. T. Nakamura, J. Koga, T.Zh. Esirkepov, et al., "High power $\gamma$-ray flash generation in ultraintense laser-plasma interactions," Phys. Rev. Lett., 108, 195001 (2012).

2. C.P. Rigers, C.S. Brady, R. Duclous, et al., "Dense electron-positron plasmas and ultraintense $\gamma$-rays from laser-irradiated solids," Phys. Rev. Lett., 108, 165006 (2012).

3. T. Nakamura and T. Hayakawa, "Numerical modeling of quantum beam generation from ultra-intense laser-matter interactions," Laser Part. Beams, 33, 151 (2015).

4. T. Nakamura and T. Hayakawa, "Laser-driven $\gamma$-ray, positron, and neutron source from ultraintense laser-matter interactions," Phys. Plasmas, 22, 083113 (2015). 


\title{
Pulsed Neutron Sources by Compton Gamma-ray Beams
}

\author{
Kenji Mishima $^{1}$ \\ ${ }^{1}$ High Energy Accelerator Research Organization, Tokai, Ibaraki, Japan \\ kenji.mishima@kek.jp
}

Neutrons are widely used for material sciences, imaging, fundamental physics and so on due to its unique properties, hence demand for more intense neutron sources is growing. Recently, spallation neutron sources using megawatt class accelerators [1-3] are coming up as a nextgeneration neutron source instead of research reactors. A spallation neutron source irradiates proton beam with energy of a few $\mathrm{GeV}$ into a neutron spallation target made of a heavy elemental material. Neutrons are produced via spallation reaction with kinetic energy of several $\mathrm{MeV}$, and were cooled down in a moderator to $1 \sim 100 \mathrm{meV}$, which are commonly used as cold or thermal neutrons. Produced neutrons have pulse structure. This nature is applied for TOF analysis, which is essentially outperform reactor neutron sources. However, the spallation neutron source needs huge neutron shield about 10-m diameter. It makes the neutron facilities large and costly, and also prevent to approach to the neutron source. Since both neutron target and accelerator are closing to the engendering limit, higher power neutron sources are getting more difficult. Thus, more effective way to produce neutrons is expected.

Inverse Compton scattering (ICS) gamma can produce very intense, sharp energy spreading gamma rays in a short timing [4-6]. Photo-neutron production reaction using ICS gamma beam can be a candidate of a new neutron source which works without moderator to realize short timing and compactness. In this talk, I will report evaluation of performance of the photo-neutron source and possible applications. I will also review recent related topics.

1. Maekawa, Fujio, et al. "First neutron production utilizing J-PARC pulsed spallation neutron source JSNS and neutronic performance demonstrated." NIMA 620.2 (2010) 159-165.

2. Mason, T. E., et al. "The Spallation Neutron Source in Oak Ridge: A powerful tool for materials research." Physica B: Condensed Matter 385 (2006) 955-960.

3. Yeck, Jim. "Neutron facility: European Spallation Source is on track." Nature 519.7543 (2015) 291-291.

4. Hajima, R. "Generation Of High-Brightness Gamma-Rays From Energy-Recovery Linac" Proceedings of ERL2011 (2012) WG2012.

5. Shimada, M., and Hajima, R. "Inverse Compton scattering of coherent synchrotron radiation in an energy recovery linac." Physical Review Special Topics-Accelerators and Beams 13.10 (2010) 100701.

6. Shimada, M., et al. "Proposal of polarized gamma-ray source for ILC based on CSR inverse Compton scattering." Proc. of IPAC 13 (2013) 1598. 


\title{
Demonstrations of neutron generation using solid-nanoparticle explosions driven by DPSSL-pumped femtosecond laser pulse
}

\author{
Takeshi Watari, Takashi Sekine, Koji Matsukado, Yasuki Takeuchi, \\ Yuma Hatano, Ryo Yoshimura, Nakahiro Satoh and Toshiyuki Kawashima, \\ Hamamatsu Photonics K.K., 1820, Kurematsu-cho, Nishi-ku, Hamamatsu City, \\ Shizuoka Pref., Japan, \\ takeshi.watari@lsr.hpk.co.jp,t-sekine@.crl.hpk.co.jp,matsukado@.crl.hpk.co.jp, \\ yasuki.takeuchi@lsr.hpk.co.jp,y-hatano@,crl.hpk.co.jp,r-yoshimura@,crl.hpk.co.jp, \\ naka@.crl.hpk.co.jp, kawasima@,crl.hpk.co.jp
}

Recent developments of high-intensity laser enable us to evolve a new type neutron source. A number of experiments [1-5] demonstrated the possibility of laser-driven fusion using pure $\mathrm{D}_{2}$ or $\mathrm{CD}_{4}$ clusters. In these works, multi-keV deuterium ions were generated by Coulomb explosion of a few nanometres clusters. Since the cross-section of deuterium-deuterium (DD) reaction reaches its maximum at $1.75 \mathrm{MeV}$ with center-of-mass system, much higher ion energy is required for efficient neutron generation. Features of the Coulomb explosion are that ion energy distribution function is proportional to the square root of its energy and the maximum energy is proportional to its radius [6]. Larger particles result in higher ion energy, although an intense laser irradiation is required for expelling electrons from the larger particles. To demonstrate high efficiency neutron generation using such a higher energy ion, we developed DPSSL-pumped high-repetition-rate 20-TW laser system and solid-nanoparticle target.

We fabricated the solid density deuterated-polystyrene (CD) nanoparicles of $250 \mathrm{~nm}$ in diameter to obtain high ion energy of $\sim 1 \mathrm{MeV}$. The average size can be controlled with high precision between 100-700 $\mathrm{nm}$. Efficient and stable neutron generation was obtained by irradiating an intense femtosecond laser pulse of $>5 \times 10^{18} \mathrm{~W} / \mathrm{cm}^{2}$. A yield of $\sim 10^{5}$ neutrons per shot was stably observed during $0.1-1 \mathrm{~Hz}$ continuous operation.

In addition, we have started demonstration of fast-neutron imaging. Fast-neutron imaging is promising application of laser induced neutron source, which has high-repetition rate and very short pulse duration $(<10 \mathrm{~ns})$. We have developed prototype of fast-neutron imaging system and demonstrate it using conventional neutron source.

1. T. Ditmire et al., "Nuclear fusion from explosions of femtosecond laser-heated deuterium clusters", Nature, 398, 489 (1999)

2. T. Ditmire et al., "Nuclear fusion in gases of deuterium clusters heated with a femtosecond laser", Phys. Plasmas, 7, 1993 (2000)

3. K. W. Madison et al., "Investigation of fusion yield from exploding deuterium-cluster plasmas produced by 100-TW laser pulses", J. Opt. Soc. Am. B, 20, 113 (2003)

4. K. W. Madison et al., "Role of laser-pulse duration in the neutron yield of deuterium cluster targets", Phys. Rev. A, 70, 053201 (2004)

5. R. Hartke et al., "Fusion neutron detector calibration using a table-top laser generated plasma neutron source", Nucl. Instrum. Methods Phys. Res. A, 540, 464 (2005)

6. K. Nishihara et al., "High energy ions generated by laser driven Coulomb explosion of cluster", Nucl. Instrum. Methods Phys. Res. A, 464, 98 (2001) 


\section{Laser Acceleration Studies in KPSI}

Kiminori Kondo ${ }^{1}$, Masaki Kando ${ }^{1}$, Mamiko Nishiuchi ${ }^{1}$, Hiromitsu Kiriyama ${ }^{1}$, Toshiyuki Shirai ${ }^{2}$, Koji Noda ${ }^{2}$ and Wataru Utsumi ${ }^{1}$

${ }^{1}$ Kansai Photon Science Institute, National Institutes for Quantum and Radiological Science and Technology, 8-1-7 Umemidai, Kizugawa, Kyoto, 619-0215, Japan

kondo.kiminori@qst.go.jp,kando.masaki@qst.go.jp,nishiuchi.mamiko@.qst.go.jp, kiriyama.hiromitsu@qst.go.jp,utsumi.wataru@qst.go.jp

${ }^{2}$ National Institute of Radiological Sciences, National Institutes for Quantum and Radiological Science and Technology, 4-9-1 Anagawa, Inage, Chiba, 263-8555, Japan shirai.toshiyuki@qst.go.jp,koji.noda@qst.go.jp

Petawatt (PW) CPA laser system is a common tool for high intensity laser physics researchers nowadays. As the application of PW lasers, laser driven charged particle acceleration, not only electrons but also hadrons, is one of the most attractive topics. In KPSI (Kansai Photon Science Institute), there are two target chambers for our PW laser J-KAREN-P. One is for studying extreme high field interaction with $\mathrm{a}_{0}$ could be $\sim 70$ using rather shorter focal length optics. This will be used for generating higher energy protons by TNSA as the first step. The other one is for generation of energetic electrons and coherent $\mathrm{x}$-rays with keeping the relativistic high field as long as possible using rather longer focal length optics.

In 2015 fall, the previous $200 \mathrm{TW}$ with 30 min interval laser system J-KAREN has been once shutdown. All the related system including the interaction target chambers has started to be improved to an upgraded one, which can yield $1 \mathrm{PW}$ on target at $0.1 \mathrm{~Hz}$ called J-KAREN-P system[1]. At present, the final commissioning is under going. As is described above, JKAREN-P will be used to generate over $\sim 100 \mathrm{MeV}$ protons for checking the system potential of generating $\mathrm{a}_{0} \sim 70$. This spring a preliminary proton acceleration experiment with J-KAREN-P was held, while the peak power was half PW on target. The chromatic aberration problem and/or rather incomplete contrast tuning gave us up to $30 \mathrm{MeV}$ in TNSA (Target Normal Sheath Acceleration) proton generation, while over $40 \mathrm{MeV}$ has been observed just before upgrade[2]. Now the chromatic aberration problem has been solved with replacing some optics in the system. The target shot experiment will start again from 2016 autumn.

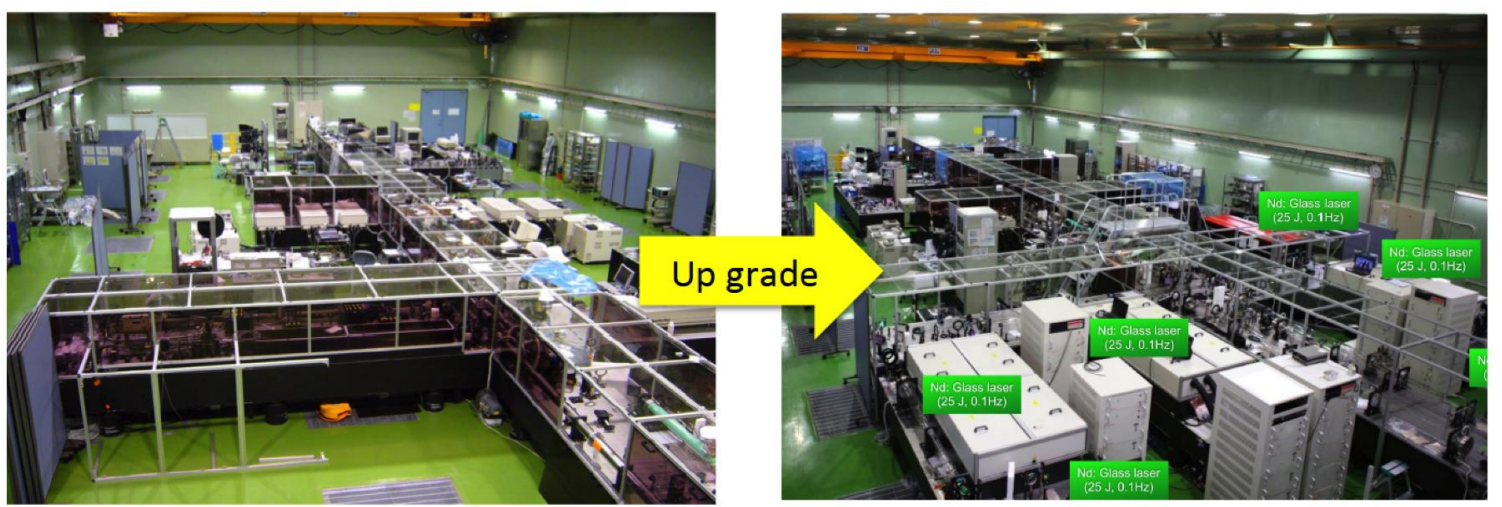

Fig. 1 Upgrade of laser amplifiers 


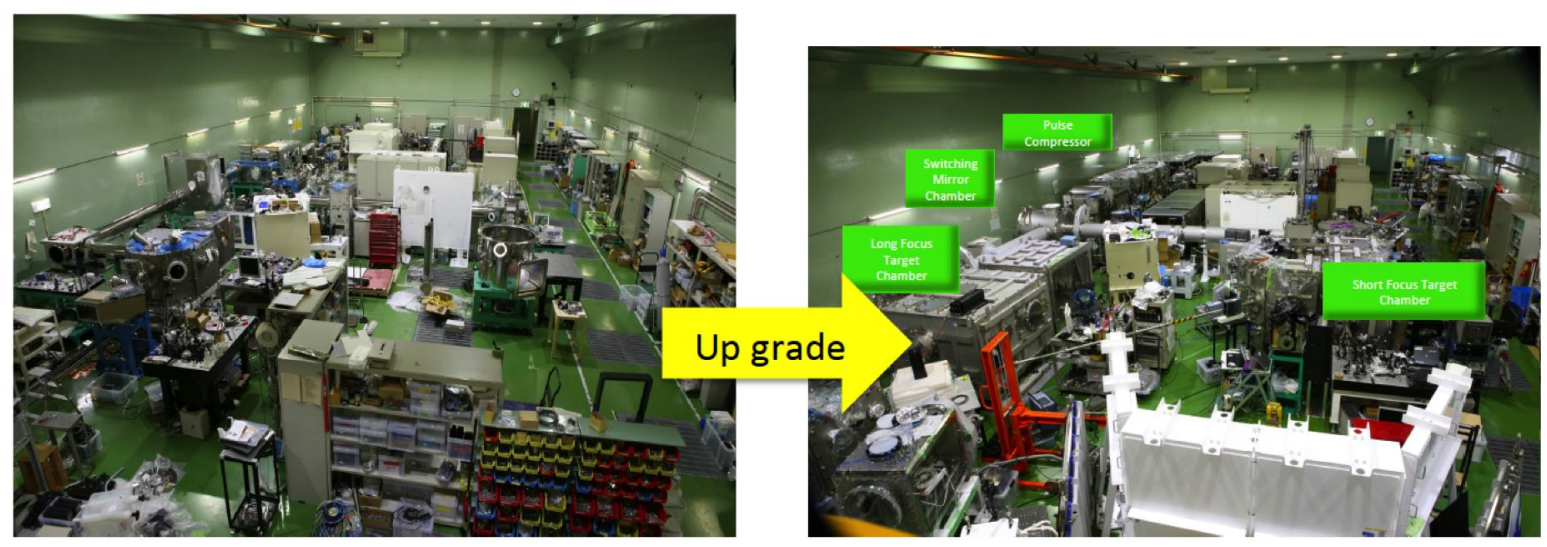

Fig. 2 Upgrade of target chambers

After the enough commissioning of J-KAREN-P, this system will be again open for users. Also the heavy ion acceleration study will be started for the development of the injector for a heavy ion synchrotron accelerator dedicated to ion cancer therapy. Actually KPSI has been moved from JAEA (Japan Atomic Energy Agency), and unified with NIRS (National Institute of Radiological Sciences) at April 1, 2016. The new society QST (National Institutes for Quantum and Radiological Science and Technology) has made a plan of the development of an advanced heavy ion accelerator for cancer therapy. We call it "Fifth generation ion cancer therapy machine". In this system, over $4 \mathrm{MeV} / \mathrm{u}$ ions will be generated with $\sim 10^{9}$ particles in $0.1 \%$ b.w. using PW laser. A generation of over $4 \mathrm{MeV} / \mathrm{u}$ ions could be done because up to $16 \mathrm{MeV} / \mathrm{u}$ ions have been successfully obtained with TNSA scheme before the upgrade[3]. The larger numbers of ions will be obtained using more advanced acceleration scheme such as RPA (Radiation Pressure Dominate Acceleration) than TNSA. The RPA scheme will be studied with J-KAREN$\mathrm{P}$ after the successful commissioning.

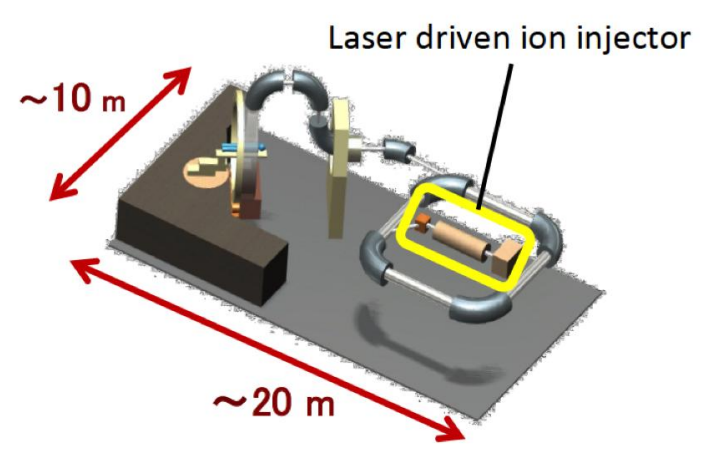

Fig. $35^{\text {th }}$ generation ion cancer therapy machine

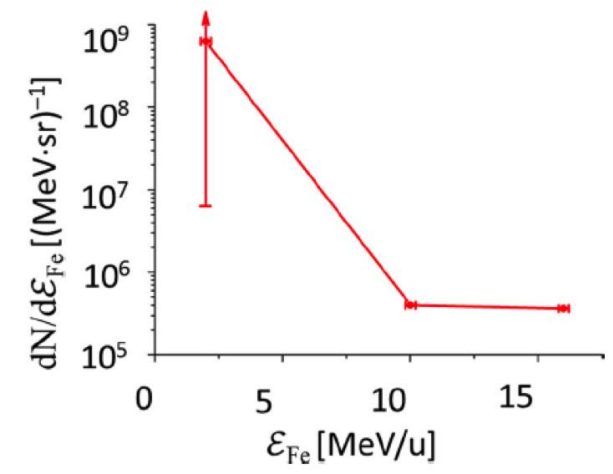

Fig. $416 \mathrm{MeV} / \mathrm{u}$ Fe ions were detected by TNSA

1. H. Kiriyama, et al., "High-contrast, high-intensity pettawatt-class laser and applications", IEEE J. QE, 21, 1601118 (2015).

2. K. Ogura, et al., "Proton acceleration to $40 \mathrm{MeV}$ using a high intensity, high contrast optical parametric chirped-pulse amplification/Ti:sapphire hybrid laser system," Opt. Lett., 37, 2868 (2012).

3. M. Nishiuchi, et al., "Acceleration of highly charged GeV Fe ions from a low-Z substrate by intense femtosecond laser," Phys. of Plasmas, 22, 033107 (2015). 


\title{
Production of 10 to $75 \mathrm{MeV}$ Compton gamma-rays from a $2 \mathrm{GeV}$ laser-plasma electron accelerator
}

\author{
Mike Downer ${ }^{1}$, J. M. Shaw ${ }^{1}$, A. C. Bernstein ${ }^{1}$, R. Zgadzaj ${ }^{1}$, Y. Chang ${ }^{1}$, A. Hannasch ${ }^{1}$, K. \\ Weichman $^{1}$, M. LaBerge ${ }^{1}$, H. E. Tsai ${ }^{1}$, T. Ditmire ${ }^{1}$, M. Donovan ${ }^{1}$, E. Gaul ${ }^{1}$, \\ ${ }^{1}$ The University of Texas at Austin, Austin, TX, USA \\ downer@physics.utexas.edu,jms8488@utexas.edu,abernste@mail.utexas.edu, \\ rafal@physics.utexas.edu,vinneko@physics.utexas.edu, andrea.hannasch@yahoo.com, \\ kweichman@utexas.edu,laberge.max@gmail.com,chicofree@hotmail.com, \\ tditmire@physics.utexas.edu, mdonovan@physics.utexas.edu,gaul@physics.utexas.edu
}

Generation of collimated, narrow bandwidth $\gamma$-ray beams by Compton backscatter (CBS) from relativistic mono-energetic electron beams opens applications in the areas of radiation therapy, photonuclear spectroscopy and homeland security. Recently, tabletop laser-plasma accelerators (LPAs) have accelerated electrons quasi-monoenergetically to 2-4 $\mathrm{GeV}$ [1-3], opening the possibility of compact CBS $\gamma$-ray sources at small university laboratories. So far, however, CBS x-rays have been generated only from $s u b-\mathrm{GeV}$ LPAs, either by backscattering a split-off laser pulse [4,5], or by retroreflecting the spent LPA drive pulse onto trailing accelerated electrons with a plasma mirror (PM) placed at the LPA exit [6,7]. The latter method is selfaligning, and recently yielded high-quality tunable (75-200 keV) x-rays from an electron-energytunable (50-90 MeV) terawatt-laser-driven LPA [7], but required a high-contrast $\left(\geq 10^{5}\right.$ at $\left.1 \mathrm{ps}\right)$ transmitted LPA drive pulse to avoid pre-expanding the PM. Since contrast of the spent drive pulse after the LPA is difficult to predict and control, scalability of the PM retro-reflection CBS method to petawatt (PW)-laser-driven multi-GeV LPAs was uncertain.

Here we report robust production of CBS $\gamma$-rays that penetrate several $\mathrm{cm}$ of lead by retroreflecting PW pulses with a PM placed after the exit of a $5 \mathrm{~cm}$ long LPA of ambient plasma electron density $n_{\mathrm{e}}=5 \times 10^{17} \mathrm{~cm}^{-3}$ that produces $\sim 100 \mathrm{pC}$ of electrons up to $2 \mathrm{GeV}$ energy [1]. We observed high $(>50 \%)$ reflectivity from the PM, and consistent $\gamma$-ray production on every shot that produced $\mathrm{GeV}$ electrons, demonstrating that high contrast at $\sim 1 \mathrm{ps}$ is maintained after the LPA. The divergence $(\sim 1 / 2 \mathrm{mrad})$ of the CBS $\gamma$-rays is much less than that $(10-20 \mathrm{mrad})$ of co-propagating betatron x-rays, which the lead filter blocks, but is close to that of the $2 \mathrm{GeV}$ electrons, suggesting that careful analysis of the $\gamma$-ray profile may measure the electron-beam emittance. We observed no CBS $\gamma$-ray signal when the PM was removed.

We estimate $10^{8}$ Compton photons are generated per shot, with peak brightness $10^{21}$ photons $/ \mathrm{s} / \mathrm{mm}^{2} / \mathrm{mrad}^{2} / 0.1 \% \mathrm{BW}$, based on analysis of measured $\mathrm{e}^{+} \mathrm{e}^{-}$pair-production efficiency using Geant4 simulations [8]. In planned research, we will implement a $\gamma$-ray spectrometer based on magnetic spectrometry of electrons that the $\gamma$-rays produce in low-Z materials [9]. The results provide a bright, directional, ultrafast $\gamma$-ray source for photonuclear spectroscopy in small university laboratories. If there are multiple authors from the same organization, only list the organization once, but include all email addresses.

1. X. Wang et al., "Quasi-monoenergetic laser-plasma acceleration of electrons to $2 \mathrm{GeV}$," Nat. Commun. 4, 1988 (2013). 
2. H. T. Kim et al., "Enhancement of electron energy to the multi-GeV regime by a dual-stage laser-wakefield accelerator pumped by petawatt laser pulses," Phys. Rev. Lett. 111, 165002 (2013).

3. W. P. Leemans et al., "Multi-GeV electron beams from capillary-discharge-guided subpetawatt laser pulses in the self-trapping regime," Phys. Rev. Lett. 113, 245002 (2014).

4. N. D. Powers et al., "Quasimonoenergetic and tunable x-rays from a laser-driven Compton light source," Nat. Photonics 8, 28-31 (2014).

5. G. Sarri et al., "Ultrahigh brilliance multi-MeV $\gamma$-ray beams from nonlinear relativistic Thomson scattering," Phys. Rev. Lett. 113, 224801 (2014).

6. K. Ta Phuoc et al., “All-optical Compton gamma-ray source,” Nat. Photonics 6, 308 (2012).

7. H.E. Tsai et al., "Compact tunable Compton x-ray source from laser-plasma accelerator \& plasma mirror," Phys. Plasmas 22, 023106 (2015).

8. J. Allison, "Geant4 developments \& applications," IEEE Trans. Nucl. Sci. 53, 270-278 (2006).

9. D. J. Corvan et al., "Design of a compact spectrometer for high-flux MeV gamma-ray beams," Rev. Sci. Instrum. 85, 065119 (2014). 


\section{Page Intentionally Left Blank}

Proc. of SPIE Vol. 10419 1041901-108

Downloaded From: https://www.spiedigitallibrary.org/conference-proceedings-of-spie on 26 Apr 2023 Terms of Use: https://www.spiedigitallibrary.org/terms-of-use 


\section{Thursday, October 20, 2016 \\ Nuclear Security \& Materials Management: 11:10 12:50 \\ Session Chair: A. Tonchev}




\title{
"Non-destructive Testing for Control of Radioactive Waste - Overview of Challenges"
}

\author{
Stéphane Plumeri ${ }^{1}$ \\ ${ }^{I}$ Andra (National radioactive waste management agency), Chatenay-Malabry, France \\ stephane.plumeri@andra.fr
}

Safety of interim storage and final disposal of radioactive waste depends strongly on characterization and quality control of the waste. As stated in [1] "Proper control of chemical and radiochemical parameters of radioactive waste within the entire waste management life cycle, and careful testing of the quality of final waste forms and waste packages, are principal components in any waste management strategy. Failure in control procedures at any step can cause important consequences, not only in follow-up steps, but, in some cases, may result in generating waste packages which are not compliant with the waste acceptance criteria for long term storage or disposal".

The characterization of conditioned radioactive waste is a specific issue because unlike for raw waste, its characterization is more complex and needs specifics non-destructive techniques and methodologies. There are different and varying reasons for this: 1) conditioned waste may not be any more in its initial form (e.g., due to incineration), 2) conditioned waste is typically embedded or surrounded by a matrix, 3) conditioned waste may contain wastes coming from different primary sources and therefore the radiological spectrum might become more complex.

Specific issues for conditioned radioactive waste are mainly characterization of radioactive waste held in large volume compounds potentially containing hidden components, spent fuels held in large volume storage containers, problematic and legacy waste, specific waste arising from repair, maintenance or decommissioning/dismantling waste and radioactive waste destined for geological disposal.

Taking into account critical parameters to be measured for each waste type and the commonly used evaluation techniques, some problematic cases arise for radioactive waste package characterization.

For example, the relatively large amount of legacy and large volume nuclear waste is a concern. The content of such packages currently cannot be characterized non-destructively with classical and standard methods since the volumes are inappropriately large or too heterogeneous for gamma or neutron scanning and imaging. In addition, hidden alpha and beta emitters contribute to the nuclear heat generation but go largely unaccounted for or are subsumed in the uncertainty assessment unless they leach out from the waste compound, which is in any case undesirable.

Therefore some technical challenges for non-destructive characterization of radioactive waste exist and innovative technics have to be developed to complement existing widely-used methods.

1. IAEA-TECDOC-1537, "Strategy and Methodology for Radioactive Waste Characterization". 


\title{
Using Prompt Neutron Angular Distributions from Polarized Photofission to Assay Special
} Nuclear Material

\author{
J. M. Mueller ${ }^{1,2}$, M. W. Ahmed ${ }^{2,3}$, H. R. Weller ${ }^{2,4}$ \\ ${ }^{1}$ Department of Nuclear Engineering, North Carolina State University, Raleigh, NC 27695, USA, \\ jonathan_mueller@ncsu.edu \\ ${ }^{2}$ Triangle Universities Nuclear Laboratory,Durham, NC 27710,USA, ahmed@tunl.duke.edu, \\ weller@tunl.duke.edu \\ ${ }^{3}$ Department of Mathematics and Physics, North Carolina Central University, Durham, NC \\ 27707, USA \\ ${ }^{4}$ Department of Physics, Duke University, Durham, NC 27708, USA
}

Recent experiments have demonstrated that the angular distributions of prompt neutrons from photofission are highly sensitive to the enrichment of the special nuclear material (SNM) under interrogation. Photofission experiments were performed on targets of ${ }^{232} \mathrm{Th},{ }^{233,235,238} \mathrm{U}$, ${ }^{237} \mathrm{~Np}$, and ${ }^{239,240} \mathrm{Pu}$ using nearly $100 \%$ linearly polarized, high intensity, and nearlymonoenergetic $\gamma$-ray beams having energies between 5.3 and $7.6 \mathrm{MeV}$ at the High Intensity $\gamma$-ray Source $(\mathrm{HI} \gamma \mathrm{S})$ located at Duke University and Triangle Universities Nuclear Laboratory [1, 2]. An array of 12-18 liquid scintillators was used to measure prompt fission neutron yields parallel and perpendicular to the plane of beam polarization. Polarization asymmetries, the differences between the in-plane and out-of-plane yields divided by their sums, were measured. Asymmetries close to zero were found for ${ }^{233,235} \mathrm{U},{ }^{237} \mathrm{~Np}$, and ${ }^{239} \mathrm{Pu}$ while significant asymmetries $(\sim 0.2-0.5)$ were found for ${ }^{232} \mathrm{Th},{ }^{238} \mathrm{U}$, and ${ }^{240} \mathrm{Pu}$. Predictions of the polarization asymmetries based on previously measured photofission fragment angular distributions combined with a model of prompt neutron emission agree well with the experimental results.

The capability of this technique to measure the enrichment of uranium was tested by using combinations of thin ${ }^{235} \mathrm{U}$ and ${ }^{238} \mathrm{U}$ foils of known enrichments [3]. Additionally, the sensitivity of this assay to shielding by lead, steel, and polyethylene was experimentally measured and simulated using GEANT4. These tests demonstrate that the measured asymmetry can indeed be used to determine the enrichment of materials composed of an admixture of ${ }^{235} \mathrm{U}$ and ${ }^{238} \mathrm{U}$, and this asymmetry is relatively insensitive to moderate amounts of shielding, making it an attractive candidate for active interrogation of SNM.

1. J. M. Mueller et al., "Prompt neutron polarization asymmetries in photofission of ${ }^{232} \mathrm{Th}$, ${ }^{233,235,238} \mathrm{U},{ }^{237} \mathrm{~Np}$, and ${ }^{239,240} \mathrm{Pu}$ ", Phys. Rev. C, 89, 034615 (2014).

2. J. M. Mueller, M. W. Ahmed, H. R. Weller, "A novel method to assay special nuclear materials by measuring prompt neutrons from polarized photofission", Nucl. Instrum. Meth. $A, \mathbf{7 5 4}, 57$ (2014).

3. J. M. Mueller et al., "Tests of a novel method to assay SNM using polarized photofission and its sensitivity in the presence of shielding”, Nucl. Instrum. Meth. A, 776, 107 (2015). 
Prof. Hideaki Ohgaki, Kyoto University - Japan

NRF based Nondestructive Inspection System for SNM using Laser-Compton Gamma-rays

The nuclear resonance fluorescence (NRF) is an attractive nondestructive analysis method because it provides signatures for a wide variety of materials with rather high photon energies. One of application of NRF technique, a detecting special nuclear materials (SNMs) within cargo containers was proposed by Bertozzi et al. and have been developed by several organizations. Our group also proposed a laser Compton backscattering gamma-ray source (LCS), which gives a quasi-monochromatic photon beam, in coupling with NRF for nondestructive inspection system of SNMs hidden in sea cargos under a program of Japan Science and Technology Agency in Japan. Although the NRF is potentially powerful for detection of hidden SNMs, it needs an acceptable inspection time for a realistic application. Therefore, an idea to combine with photons and neutrons has been proposed and developed. The developed inspection system consists of an active neutron detection system for a fast screening and an LCS with NRF method for a precise inspection. A race-track microtron has been used to make the system into compact. For the NRF measurement, an array of $\mathrm{LaBr} 3(\mathrm{Ce})$ scintillation detectors has been adopted to realize a low-cost detection system. The prototype system has been constructed and demonstration measurement has been conducted. As a result, we have concluded that the designed system can detect some amount of highly enriched $235 \mathrm{U}$ (HEU) hidden in a $20-\mathrm{ft}$ container within a realistic inspection time. 


\title{
Photonuclear Reactions for Neutrino-Nuclear Responses and Nuclear Isotope Detection
}

\author{
Hiro Ejiri ${ }^{1}$ \\ ${ }^{1}$ RCNP, Osaka University Osaka 5670047 Japan \\ ejiri@rcnp.osaka-u.ac.jp
}

Neutrino (weak) nuclear responses for double beta decays and astro neutrinos are crucial for neutrino studies and neutrino-induced nucleosynthesis. They are studied by photonuclear reactions via isobaric analogue states [1,2]. The photonuclear electric and magnetic gammatransitions give corresponding vector and axial vector weak responses. The photonuclear cross sections give the weak responses, while angular distributions of neutrons following the photo nuclear reactions give the spin parity of the relevant state [3].

RPID (Resonant Photonuclear Isotope Detection) is high-sensitivity investigation of gamma rays from residual radio-active isotopes produced by photonuclear reactions via giant resonance [4]. It is used to identify ppt-ppb level nuclear-isotopes for astro neutrino and applied science.

High quality polarized photons produced by Compton back-scattering off $\mathrm{GeV}$ electrons are very useful for studies of these neutrino responses and nuclear isotope detection. [3,4].

1. H. Ejiri, "Double beta decays and neutrino mases," J.Phys.Soc.Japan, 74, 2101 (2005).

2. H. Ejiri and J. Suhonen, "GT neutrino-nuclear responses for double beta decays and astro neutrinos," J. Phys. G, 42, 055201 (2015).

3. H. Ejiri et al., "Neutrino-nuclear responses and photonuclear reactions," Phys. Rev. C, 88, 054601 (2013).

4. H. Ejiri and T. Shima, "Resonant photonuclear isotope detections using medium energy photonuclear reactions," Phys. Rev. ST, 15024701 (2012). 


\section{Page Intentionally Left Blank}

Proc. of SPIE Vol. 10419 1041901-114

Downloaded From: https://www.spiedigitallibrary.org/conference-proceedings-of-spie on 26 Apr 2023 Terms of Use: https://www.spiedigitallibrary.org/terms-of-use 


\section{Thursday, October 20, 2016 \\ Enabling Technologies for Nuclear Photonics: 14:15 16:00 \\ Session Chair: F. Albert}




\section{Laser-driven multi-modal beams for nuclear waste management inspection}

C M Brenner ${ }^{1}$, C P Jones, T B Scott, J Jowsey, S R Mirfayzi ${ }^{5}$, D R Rusby ${ }^{1,4}$, C Armstrong ${ }^{1,4}$, A Alejo $^{5}$, L A Wilson ${ }^{1}$, R Clarke ${ }^{1}, \mathrm{H}_{\text {Ahmed }}{ }^{5}, \mathrm{~N} \mathrm{M} \mathrm{H} \mathrm{Butler}^{4}$, D Haddock ${ }^{1}$, A Higginson ${ }^{4}$, A McClymont ${ }^{1}$, C Murphy ${ }^{6}$, M Notley ${ }^{1}$, P Oliver $^{1}$, R Allott ${ }^{1}, \mathrm{C}_{\text {Hernandez-Gomez }}{ }^{1}, \mathrm{~S} \mathrm{Kar}^{5}$, P McKenna ${ }^{4}$ and D Neely ${ }^{1}$

1. Central Laser Facility, STFC, Rutherford Appleton Laboratory, Didcot, Oxon, OX11 0QX,U.K, ceri.brenner@stfc.ac.uk,chris.armstrong@stfc.ac.uk, Lucy.wilson@stfc.ac.uk,Rob.clarke@stfc.ac.uk,David.haddock@stfc.ac.uk, Margaret.notley@stfc.ac.uk, ric.allott@stfc.ac.uk,Cristina.hernandez-gomez@stfc.ac.uk, david.neely@stfc.ac.uk

2. Interface Analysis Centre, HH Wills Physics Laboratory, Tyndall Avenue, Bristol, BS8 1TL,UK,cj0810@bristol.ac.uk,phtbs@bristol.ac.uk,

3. Ground Floor North B582, Sellafield Ltd, Seascale, Cumbria CA20 1PG, john.jowsey@sellafieldsites.com

4. Department of Physics, SUPA, University of Strathclyde, Glasgow G4 0NG, U.K., nicholas.butler.2014@uni.strath.ac.uk,adam.higginson.2013@uni.strath.ac.uk, paul.mckenna@strath.ac.uk

5. Centre for Plasma Physics, Queen's University Belfast, Belfast BT7 1NN, U.K., smirfayzi01@qub.ac.uk,aalejo01@qub.ac.uk,h.ahmed@qub.ac.uk,s.kar@qub.ac.uk 6. Department of Physics, University of York, YorkYO105DD,U.K., chris.murphy@york.ac.uk

High-power ( $>100 \mathrm{TW}$ ) laser-solid interactions drive micro-scale electron accelerators that can generate bright, point-like sources of high-energy x-rays, ions and neutrons. These laser-driven beams are of particular interest for industrial and security applications where imaging and/or inspection through large and dense objects is required.

At the UK's Central Laser Facility (CLF) the Vulcan laser has been operational at petawatt level for over a decade to study the underlining physics behind the beam generation and in recent years has also been used to demonstrate their potential for applications across many high-value sectors. The CLF is now developing high repetition rate capability, via its novel DiPOLE system - a high average power, diode-pumped laser system capable of delivering $100 \mathrm{~J}$ pulses at $10 \mathrm{~Hz}$.

A small scale sample nuclear waste package, consisting of a $28 \mathrm{~mm}$ diameter uranium penny encased in grout, was imaged by absorption contrast radiography using a single pulse exposure from an x-ray source driven by a high-power laser. The Vulcan laser was used to irradiate a tantalum foil, in order to generate a bright burst of highly penetrating x-rays (with energy $>500 \mathrm{keV}$ ), with a source size of $<0.5 \mathrm{~mm}$. BAS-TR and BAS-SR image plates were used for image capture, alongside a newly developed large area Thalium doped Caesium Iodide scintillator-based detector coupled to CCD chips. The uranium penny was clearly resolved to sub-mm accuracy over a $30 \mathrm{~cm}^{2}$ scan area from a single shot acquisition. In addition, neutron generation was demonstrated in situ with the x-ray beam, with a single shot, thus demonstrating the potential for multi-modal criticality testing of waste materials. This feasibility study successfully demonstrated non-destructive radiography of encapsulated, high density, nuclear material.

The CLF is partnering on a 3-year project with University of Bristol, Queen's University Belfast and Sellafield Ltd to further demonstrate proof of concept imaging and inspection with 
realistic samples relevant to nuclear waste monitoring. Recent developments of high-power laser systems at the CLF, to $10 \mathrm{~Hz}$ operation, allows for a laser-driven multi-modal beamline for waste monitoring applications to be envisioned.

1. C. M. Brenner et al, "Laser-driven x-ray and neutron source development for industrial applications of plasma accelerators" Plasma Phys. Control. Fusion, 58, 014039 (2016).

2. C. P. Jones, C. M. Brenner et al, "Evaluating laser-driven Bremsstrahlung radiation sources for imaging and analysis of nuclear waste packages", Journal of Hazardous Materials, 318, 694-701 (2016) 


\title{
Compact, High Brightness Accelerators for Laser-Compton Sources
}

\author{
Roark A. Marsh \\ Lawrence Livermore National Laboratory, Livermore, CA, USA \\ marsh19@,llnl.gov
}

The brightness of a successful Laser-Compton source follows directly from the quality of the electron beam. Electron beam quality is fundamentally tied to the initial particle distribution and constrained to degrade as little as possible through subsequent manipulations. The high brightness radiofrequency photoinjector has made the current generation of FELs and advanced light sources possible, and continues to produce incredibly bright bunches of electrons [1]. Photoinjectors are capable of reliably producing high charge, small emittance, and low energy spread. A high gradient linear accelerator follows the photoinjector in order to boost the electron energy to produce the desired final gamma-ray energy in a compact footprint [2]. Multiple electron bunches or bunch trains can also be utilized to effectively increase the total beam current and therefore the average photon flux [3]. I will discuss the design of a $250 \mathrm{MeV}$ all X-band accelerator and its predicted performance [4], the scaling of this design up in energy to reach $20 \mathrm{MeV}$ gamma-rays, and down in energy to produce $200 \mathrm{keV}$ x-rays [5]. I will discuss current electron beam achievements from the state-of-the-art X-band accelerator at LLNL, and the excellent agreement between Laser-Compton code predictions and the performance of the X-band Compton Light Source [6]. I will discuss future directions and the scaling of current results along planned upgrade routes.

This work was performed under the auspices of the U.S. Department of Energy by Lawrence Livermore National Laboratory under Contract DE-AC52-07NA27344.

[1] R. Akre, D. Dowell, P. Emma, J. Frisch, S. Gilevich, G. Hays, Ph. Hering, R. Iverson, C. Limborg-Deprey, H. Loos, A. Miahnahri, J. Schmerge, J. Turner, J. Welch, W. White, and J. Wu, "Commissioning the Linac Coherent Light Source injector" Phys. Rev. ST Accel. Beams 11, 030703 (2008).

[2] F. V. Hartemann, W. J. Brown, D. J. Gibson, S. G. Anderson, A. M. Tremaine, P. T. Springer, A. J. Wootton, E. P. Hartouni, and C. P. J. Barty, "High-energy scaling of Compton scattering light sources" Phys. Rev. ST Accel. Beams 8, 100702 (2005)

[3] D. J. Gibson, G. G. Anderson, S. G. Anderson, R. A. Marsh, M. J. Messerly, M. A. Prantil, C. P. J. Barty, “GHz pulse-train X-band capability for laser Compton x-ray and $\gamma$-ray sources", Proceedings of the International Particle Accelerator Conference, Richmond, VA, USA TUBC2 (2015)

[4] S.G. Anderson, F. Albert, A.J. Bayramian, G. Beer, R.E. Bonanno, R.R. Cross, G. Deis, C.A. Ebbers, D.J. Gibson, F.V. Hartemann, T.L. Houck, R.A. Marsh, D.P. McNabb, M.J. Messerly, R.D. Scarpetti, M.Y. Shverdin, C.W. Siders, S.S. Wu, C.P.J. Barty, C.E. Adolphsen, T.S. Chu, E.N. Jongewaard, Z. Li, C. Limborg, S.G. Tantawi, A.E. Vlieks, F. Wang, J.W. Wang, F. Zhou, T.O. Raubenheimer, "VELOCIRAPTOR: An Xband photoinjector and linear accelerator for the production of Mono-Energetic $\gamma$-rays", Nuclear Instruments and Methods in Physics Research Section A, 657, 1, Pages 140-149 ( 2011)

[5] R. A. Marsh, F. Albert, S. G. Anderson, G. Beer, T. S. Chu, R. R. Cross, G. A. Deis, C. A. Ebbers, D. J. Gibson, T. L. Houck, F. V. Hartemann, C. P. J. Barty, A. Candel, E. N. Jongewaard, Z. Li, C. Limborg-Deprey, A. E. Vlieks, F. Wang, J. W. Wang, F. Zhou, C. Adolphsen, and T. O. Raubenheimer, "Modeling and design of an Xband rf photoinjector", Phys. Rev. ST Accel. Beams 15, 102001 (2012)

[6] Y. Hwang, T. Tajima, G. Anderson, D. J. Gibson, R. A. Marsh, C. P. J. Barty, "LLNL laser-Compton x-ray characterization", Proceedings of the International Particle Accelerator Conference, Busan, Korea TUPOW052 (2016) 


\title{
Ultra-low Background Counting Technique on the Earth's Surface
}

\author{
Tatsushi Shima \\ Research Center for Nuclear Physics, Osaka University, Ibaraki, Osaka, Japan \\ shima@rcnp.osaka-u.ac.jp
}

The technique of ultra-low background radiation counting is essential for experimental researches with extremely low event rates in the fields of particle physics, nuclear physics, radioactivity analysis, and so on. In general, such measurements are performed with a detector surrounded by heavy shields against environmental radiations at a laboratory located deep underground where high-energy cosmic-rays are reduced by many orders of magnitude. Such kind of detectors are, however, not easy to be used in combination with advanced quantum beams which are provided at high-performance accelerators on the Earth's surface. Conversely, if one succeeds in achieving the background level at a surface laboratory as low as those in underground laboratories, it will provide a new opportunity to make ultra-low background activation measurements with advanced quantum beams.

On the Earth's surface, the background events due to cosmic-rays can be categorized into two types, one is the direct events caused by charged particles, and another is the events due to primary and secondary neutral particles in cosmic-rays. The former can be efficiently reduced by using an active shield made of appropriate veto counters. On the other hand, the latter is not easy to be suppressed with either active or passive shields. To develop a method to reduce the background induced by neutral particles, we studied the intensity and the mechanism of those background. In this paper the influence of cosmogenic $\gamma$-rays and neutrons and the feasibility of ground-based ultra-low background measurements are discussed. 


\title{
Gamma-beam monitoring with diamond sensors: fast, radiation hard and hardly destructive
}

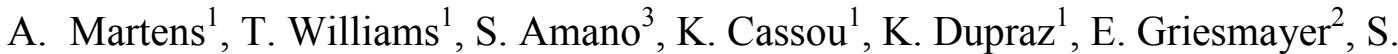 \\ Hashimoto $^{3}$, P. Kavrigin ${ }^{2}$, S. Miyamoto ${ }^{3}$, A. Takemoto ${ }^{3}$, M. Yamaguchi ${ }^{3}$, F. Zomer ${ }^{1}$ \\ ${ }^{1}$ LAL, Univ Paris-Sud, CNRS/IN2P3, Université Paris-Saclay, Orsay, France \\ martens@lal.in2p3.fr,williams@lal.in2p3.fr,cassou@lal.in2p3.fr,dupraz@lal.in2p3.fr, \\ zomer@lal.in2p3.fr \\ ${ }^{2}$ Vienna University of Technology, Austria, erich.griesmayer@cividec.at, \\ pavel.kavrigin@cividec.at \\ ${ }^{3}$ Laboratory of Advanced Science and Technology for Industry, University of Hyogo, Kouto, \\ Hyogo,Japan,sho@lasti.u-hyogo.ac.jp,hashi@lasti.u-hyogo.ac.jp,miyamoto@lasti.u- \\ hyogo.ac.jp,akinori@lasti.u-hyogo.ac.jp,ym731@lasti.u-hyogo.ac.jp
}

Operations of a diamond sensor placed in high average intensity beam of photons with energies of several $\mathrm{MeV}$ are reported [1]. Data was taken at the HI $\gamma \mathrm{S}$ facility of TUNL and newSUBARU of Hyogo University. The energies of the photons during data taking varied from $2 \mathrm{MeV}$ up to $34 \mathrm{MeV}$ with several configurations of the laser beam polarizations. The high capability to resolve trains of bunches separated by about $15 \mathrm{~ns}$ is demonstrated. The capabilities of this new sensor, in the context of gamma-ray beam sensing, for transverse beam shape measurement, flux and energy measurements are exposed. Indirect measurement of the laser polarization, and thus under certain conditions gamma-ray beam polarization, are investigated. The results indicate that the tested apparatus fulfils the requirements for a fast monitoring detector for the ELI-NP source [2,3] currently under construction, which motivates this work, and demonstrates for the first time the capabilities of such detectors in high average-intensity photon beams. This new tool may allow precise and hardly destructive photon beam monitoring for nuclear photonics.

The presentation will first briefly motivate the need for diamond sensors in gamma-ray beam sensing. Then a summary of the results previously obtained at HI $\gamma \mathrm{S}$ will be given. The main part of the talk will be devoted to new results obtained at newSUBARU.

1. T. Williams, et al., "Operation of a fast diamond $\gamma$-ray detector at the HI $\gamma \mathrm{S}$ facility," Nucl. Inst. Methods A, 830, 391 (2016).

2. O. Adriani, et al., "Technical Design Report EuroGammaS proposal for the ELI-NP Gamma beam System," arXiv:physics.acc-ph/1407.3669.

3. K. Dupraz, et al., Design and optimization of a highly efficient optical multipass system for $\gamma$-ray beam production from electron laser beam Compton scattering, Phys. Rev. ST Accel. Beams, 17, 033501 (2014). 


\title{
ELITPC - an Active-Target TPC for Studying Photonuclear Reactions with High- Brilliance Gamma Beam at the ELI-NP Facility
}

\author{
M. Cwiok ${ }^{1}$, J.S. Bihalowicz ${ }^{1}$, W. Dominik ${ }^{1}$, Z. Janas ${ }^{1}$, L. Janiak ${ }^{1}$, T. Matulewicz ${ }^{1}$, \\ C. Mazzocchi ${ }^{1}$, M. Pfützner ${ }^{1}$, P. Podlaski ${ }^{1}$, M. Zaremba ${ }^{1}$, D. Balabanski ${ }^{2}$, C. Balan ${ }^{2}$, D.G. Ghita ${ }^{2}$, \\ O. Tesileanu ${ }^{2}$, M. Gai ${ }^{3}$, D.P. Kandellen ${ }^{3}$ \\ ${ }^{1}$ University of Warsaw, Faculty of Physics, Warsaw, Poland \\ Mikolaj.Cwiok@fuw.edu.pl \\ ${ }^{2}$ IFIN-HH / ELI-NP, Bucharest-Magurele, Romania \\ ${ }^{3}$ University of Connecticut, CT, USA
}

A newly built Extreme Light Infrastructure - Nuclear Physics (ELI-NP) facility in Bucharest-Magurele, Romania will provide monochromatic, high-brilliance gamma-ray beams that will allow one to study key nuclear reactions in modern astrophysics by means of the inverse photo-dissociation process [1]. Such inverse reactions exhibit larger cross sections due to detailed balance principle and have smaller experimental backgrounds in comparison with direct measurements.

One of the benchmark reactions to be studied at ELI-NP is the ${ }^{16} \mathrm{O}(\gamma, \alpha){ }^{12} \mathrm{C}$ photodissociation process that can shed more light in explaining carbon-to-oxygen abundance ratio observed in the Universe. In order to measure this and other $(\gamma, \alpha)$ or $(\gamma, p)$ reactions of astrophysical interest, an active-target gaseous Time Projection Chamber (ELITPC) is being developed by the University of Warsaw, IFIN-HH/ELI-NP and the University of Connecticut [2].

The ELITPC detector has a drift volume of about $35 \times 20 \times 20 \mathrm{~cm}^{3}$ that is centered around the axis of the gamma beam. The working gas mixture, rich with target nuclei to be studied, is kept at a lower-than-atmospheric pressure $(\sim 100 \mathrm{mbar})$ in order to optimize $3 \mathrm{D}$ kinematical reconstruction of the events. The ionization electrons from tracks of charged particles emerging from photo-dissociation reactions drift in a uniform electric field towards several Gas Electron Multiplier (GEM) structures before reaching the segmented readout anode. The whole internal structure is embedded in a vacuum vessel equipped with gamma-beam windows, gas and highvoltage ports as well as analogue signal feedthroughs. The ELITPC detector is complemented by low-pressure generation and recirculation gas system, electron drift velocity monitoring detector and real-time gamma-beam intensity diagnostics.

The detector will employ fast digitizing front-end electronics developed by the Generic Electronics for TPCs (GET) collaboration for middle-size experiments in nuclear physics [3]. The readout anode is constituted from interconnected pads that are arranged in three arrays of strips, which form a redundant three-coordinate $u-v-w$ system. About $10^{3}$ electronic channels are envisaged in the full-scale ELITPC detector.

A scaled demonstrator detector operating at atmospheric pressure was constructed and tested with an alpha-particle beam at the IFIN-HH Tandem facility Romania [4]. The beaminduced experimental background for the expected gamma-ray intensities and energies has been simulated using Monte Carlo. The current R\&D program focuses on testing thicker versions of GEM foils that will be more suitable for operation at low gas densities, further development of FPGA-based DAQ electronics, optimizing segmentation of the readout strips and number of 
electronics channels and optimizing composition of the working gas mixture (e.g. $\mathrm{He}+\mathrm{CO}_{2}$ ). A brief status of these developments will be presented in the talk.

1. D. Filipescu et al., "Perspectives for photonuclear research at the Extreme Light Infrastructure - Nuclear Physics (ELI-NP) facility," European Physical Journal A, 51, 185 (2015).

2. O. Tesileanu et al., "Charged particle detection at ELI-NP," Romanian Reports in Physics, 68, S699 (2016).

3. E. Pollacco et al., "GET: A Generic Electronic System for TPCs for Nuclear Physics Experiments," Physics Procedia, 37, 1799 (2012).

4. M. Cwiok, "Nuclear reactions at astrophysical energies with $\gamma$-ray beams: a novel experimental approach," Acta Physica Polonica B, 47, 707 (2016). 


\section{Thursday, October 20, 2016 \\ Isotope-specific Detection, Assay and Imaging: 16:20 17:40 \\ Session Chair: A. Afanasev}




\section{Experimental Demonstrations of Isotope-specific Material Detection}

Félicie Albert

Lawrence Livermore National Laboratory

This talk will review experiments using quasi-monoenergetic gamma-rays for isotope-specific detection through nuclear resonance fluorescence.

Gamma-rays, produced by Compton scattering laser photons off relativistic $(100$ MeV-class), monoenergetic electron beams from a linac, are particularly well suited for NRF detection because of their relatively low spectral bandwidth. The isotopespecific detection of ${ }^{7} \mathrm{Li}$ in $\mathrm{LiH}$ shielded by $\mathrm{Pb}$ and $\mathrm{Al}$ was accomplished with a Compton source at LLNL using the nuclear resonance fluorescence line of ${ }^{7} \mathrm{Li}$ at 478 $\mathrm{keV}$. More recently, the technique was perfected at the ESRF synchrotron, where ESRF's ID15A beamline was used to simulate, at $478 \mathrm{keV}$, the output of light sources based on laser-Compton scattering. This output was used to assay and map the location ${ }^{7} \mathrm{Li}$ in several objects through further isotope-specific material detection and imaging. These experiments demonstrate the potential of Compton-scattering photon sources to accurately detect isotopes in situ. 


\title{
Assessment of Near-monoenergetic Photon Sources for Nonproliferation Applications
}

\author{
Bernhard A Ludewigt ${ }^{1}$, Cameron GR Geddes ${ }^{1}$, John D Valentine ${ }^{1}$, Brian Quiter $^{1}$, Marie-Anne \\ Descalle $^{2}$, Glen Warren ${ }^{3}$, Mathew Kinlaw ${ }^{4}$, Cameron Miller ${ }^{5}$ \\ ${ }^{1}$ Lawrence Berkeley National Laboratory, Berkeley, CA, USA \\ BALudewigt@lbl.gov,cgrgeddes@lbl.gov,jdvalentine@lbl.gov,bjquiter@lbl.gov \\ ${ }^{2}$ Lawrence Livermore National Laboratory,Livermore, CA, USA, descalle1@llnl.gov \\ ${ }^{3}$ Pacific Northwest National Laboratory,Richmond,WA, USA, glen.warren@pnnl.gov \\ ${ }^{4}$ Idaho National Laboratory, Idaho Falls, ID, USA, Mathew.kinlaw@inl.gov \\ ${ }^{5}$ University of Michigan, Ann Arbor,MI, USA, cmillera@umich.edu
}

Near-monoenergetic photon sources have the potential to provide significant performance enhancements or enable new capabilities in nonproliferation applications. The advantage lies in the ability to select energy, energy spread, flux and pulse structures to deliver the photons needed for signature generation while suppressing extraneous dose and background that is associated with current bremsstrahlung sources. New $\mathrm{MeV}$ photon sources based on Thomson scattering are currently under development that promise unprecedented performance with photon energies from below $1 \mathrm{MeV}$ to greater than $20 \mathrm{MeV}$, energy spreads as low as $\sim 1 \%$, and $\sim 10^{8}$ photon/pulse at repetition rates up to $10 \mathrm{~s}$ of $\mathrm{kHz}$.[1] To guide the development of such sources in the nonproliferation space, a broad range of applications, where near-monoenergetic photon sources may have a high-impact, has been identified, application requirements, current capabilities and gaps determined, and photon source performance requirements, including source tradeoffs and constraints assessed. The applications investigated included cargo screening and interdiction, single-sided inspection to detect hidden SNM, treaty/dismantlement verification, nuclear safeguards, and emergency response.

Initial studies showed that dose reductions, due to use of a narrow energy-spread source, range from about $2 \mathrm{x}$ to $4 \mathrm{x}$ for radiography applications and can exceed a factor of 10 for photofission. At energy spreads at or below the percent level, nuclear resonance fluorescence measurements could be performed at several orders of magnitude lower doses than with a bremsstrahlung beam. Additional benefit is available from sources with low beam divergence, which eliminates the need for collimators and makes it possible to deliver a high photon flux onto the target area at a larger distance. Furthermore, scanning with a narrow beam allows the scatter component in the radiograph of a thick object to be largely eliminated and thus enables further dose reduction and/or great increase in contrast. Results of a quantitative assessment of system performance, source requirements, and capability enhancement will be presented.

1. C.G.R. Geddes, et al., "Compact quasi-monoenergetic photon sources from Thomson scattering using laser-plasma accelerators and plasma channels," Nucl. Instr. Meth. B, 350, 116 (2015). 


\title{
Laser-driven Neutrons for Active Interrogation of Special Nuclear Material
}

\author{
A. Favalli \\ Los Alamos National Laboratory, Los Alamos, NM, U.S.A. \\ afavalli@lanl.gov
}

At Los Alamos National Laboratory (LANL), we have recently pioneered a novel short duration yet extremely intense neutron source using a short-pulse laser. At the Trident laser facility, one of the most intense and powerful short-pulse lasers in the world, a laser beam can be concentrated to peak intensity up to $10^{21} \mathrm{~W} / \mathrm{cm}^{2}$. The beam, interacting with an ultrathin (submicron) deuterated plastic foil target, drives a high-energy deuteron beam, which produces neutrons in a beryllium converter. This neutron source features high intensity and directionality, $>10^{10}$ fast neutrons per steradian per shot, with extremely short neutron pulse duration i.e. on the order of a few nanoseconds. One of the motivations for such a source is the capability to perform nondesctructive assay of special nuclear material for nuclear material accountancy, nuclear safeguards and national security applications. . Dedicated experimental campaigns were conducted at LANL to investigate the merits and applicability of such an approach to active interrogation of uranium and plutonium materials. Results of these measurements have provided the first of a kind experimental demonstration of active interrogation using high-intensity laserdriven neutron source and demonstrate feasibility of interrogation using a single laser-driven neutron pulse. Results obtained will be presented. 


\section{Determination of Photofission Fragment Characteristics of ${ }^{234,238} \mathrm{U}$ and ${ }^{232} \mathrm{Th}$ in the Barrier Region}

Andreas Oberstedt ${ }^{1}$, Martin Freudenberger ${ }^{2}$, Alf Göök ${ }^{2,3}$, Christian Eckardt ${ }^{2}$, Joachim Enders ${ }^{2}$, Peter von Neumann-Cosel ${ }^{2}$, Stephan Oberstedt ${ }^{3}$, and Achim Richter ${ }^{2}$

${ }^{1}$ Extreme Light Infrastructure - Nuclear Physics/Horia Hulubei National Institute for Physics and Nuclear Engineering, Bucharest-Magurele, Romania email: andreas.oberstedt@eli-np.ro

${ }^{2}$ Institut für Kernphysik, Technische Universität Darmstadt, Darmstadt, Germany

${ }^{3}$ European Commission, DG JRC, Directorate G-Nuclear Safety and Security, G.2 SN3S, Geel, Belgium

Mass and angular distributions as well as total kinetic energies (TKE) of fission fragments from light actinides have been studied with bremsstrahlung photons produced with the superconducting Darmstadt electron linear accelerator S-DALINAC. The endpoint energies of the bremsstrahlung spectra were chosen to cover the barrier regions of the investigated nuclei ${ }^{234,238} \mathrm{U}$ and ${ }^{232} \mathrm{Th}$.

The experimental results were analyzed in terms of fission modes according to the multimodal random-neck rupture model [1], and correlations between different characteristic distributions of fission fragment properties were observed and explained [2].

This work was supported by the Deutsche Forschungsgemeinschaft through SFB 634.

1. U. Brosa, S. Grossman, A. Müller, "Nuclear Scission”, Phys. Rep., 197, 167 (1990).

2. A. Göök, Dissertation, D17, Technische Universität Darmstadt (2012). 


\section{Page Intentionally Left Blank}

Proc. of SPIE Vol. 10419 1041901-128

Downloaded From: https://www.spiedigitallibrary.org/conference-proceedings-of-spie on 26 Apr 2023 Terms of Use: https://www.spiedigitallibrary.org/terms-of-use 


\section{Friday, October 21, 2016 \\ Intense Laser Driven Sources and Technology: 8:30 10:30 \\ Session Chair: D. Gibson}




\section{Progress and Prospects far Lasers at 10PW and Beyond}

Gaul, Erhard

University of Texas - USA

Proc. of SPIE Vol. $104191041901-130$

Downloaded From: https://www.spiedigitallibrary.org/conference-proceedings-of-spie on 26 Apr 2023 Terms of Use: https://www.spiedigitallibrary.org/terms-of-use 


\title{
Improving laser-driven ion acceleration for applications in nuclear physics
}

\author{
Florian Wagner $^{1}$, Markus Roth ${ }^{2}$, Vincent Bagnoud ${ }^{1}$ \\ ${ }^{1}$ GSI Helmholtzzentrum für Schwerionenforschung GmbH, Darmstadt, Germany \\ f.wagner@gsi.de,v.bagnoud@gsi.de \\ ${ }^{2}$ Technische Universität Darmstadt, Darmstadt, Germany \\ markus.roth@physik.tu-darmstadt.de
}

High power laser pulses focused to intensities exceeding $10^{18} \mathrm{~W} / \mathrm{cm}^{2}$ offer the possibility to accelerate protons and heavy ions to maximum energies up to tens of $\mathrm{MeV}$ per nucleon. Thanks to the beneficial qualities of those laser-accelerated ion beams many possible applications are currently being considered. Besides utilization in the fields of material science, medical physics, biology or high energy density physics, particularly nuclear physics could benefit from laseraccelerated ions due to their potential to trigger various kinds of nuclear reactions. Moreover, we have demonstrated that such ions could be converted into energetic directional neutron beams [1]. Thanks to the compactness and simplicity of laser-driven neutron sources, they could complement conventional neutron facilities like reactors or accelerator based spallation sources and could open the field of neutron physics to a broad community.

Most of the possible applications make high demands on absolute particle numbers, spectral qualities and especially the robustness and reproducibility of the acceleration scheme. In this contribution we discuss our recent experimental results of proton beams with cutoff energies in excess of $85 \mathrm{MeV}$ and exceptionally high particle numbers [2]. Based on our experiments and simulations we show that applying the well established target normal sheath acceleration mechanism (TNSA) with sub-micrometer thick plastic targets is a very robust approach to achieve such high ion energies and fluxes.

Furthermore great potential regarding maximum ion energies and conversion efficiency especially for heavy ions has been predicted for alternative laser based acceleration mechanisms such as breakout afterburner (BOA) or radiation pressure acceleration (RPA). Despite first promising experimental results (e.g. in [3]) the outcome still stays far behind the predictions from simulations and theoretical considerations. To overcome this discrepancy more detailed knowledge about the physical processes happening during the laser-target interaction is required. Besides measuring secondary radiation like the accelerated ions, we gain information by detecting the laser light that is respectively reflected from and transmitted through the target spectrally and temporally resolved. This yields details about the dynamics of the critical density and the onset of relativistic transparency, which is of particular interest for discrimination between the different mechanisms TNSA, BOA and RPA.

1. M. Roth et. al., "Bright laser-driven neutron source based on the relativistic transparency of solids", Phys. Rev. Lett., 110, 044802 (2013)

2. F. Wagner et. al., "Maximum proton energy above $85 \mathrm{MeV}$ from the relativistic interaction of laser pulses with micrometer thick $\mathrm{CH}_{2}$ targets“, Phys. Rev. Lett., 116, 205002 (2016)

3. $\quad$ F. Wagner et. al., "Simultaneous observation of angularly separated laser-driven proton beams accelerated via two different mechanisms“, Phys. Plasmas, 22, 063110 (2015) 


\section{High-energy charged particles by laser-plasma interactions and applications}

Kitae Lee ${ }^{1}$, Seong Hee Park ${ }^{1}$, Kyung Nam Kim ${ }^{1}$, Ha-Na Kim ${ }^{1,2}$, Woo-Je Ryu ${ }^{1,3}$, Manoj Kumar ${ }^{1}$, Shin-Yeong Lee ${ }^{1,4}$, Jeong Tae Kim ${ }^{1,4}$, Yong-Ho Cha ${ }^{5}$, Young Uk Jeong ${ }^{1}$, and Jae Hoon Kim ${ }^{6}$

${ }^{1}$ Center for Quantum-Beam-based Radiation Research, Korea Atomic Energy Research Institute, Daejeon, KOREA

kleegle@gmail.com

${ }^{2}$ Physics Department, Chungnam National University, Daejeon, KOREA

${ }^{3}$ Physics Department, Hannam University, Daejeon, KOREA

${ }^{4}$ Accelerator and Nuclear Fusion Physical Engineering, University of Science and Technology, Daejeon, KOREA

${ }^{5}$ Quantum Optics Division, Korea Atomic Energy Research Institute, Daejeon, KOREA

${ }^{6}$ Korea Electrotechnology Research Institute, Ansan, KOREA

When an ultra-intense laser pulse is focused on a target, such as thin foil or atomic gas, high-energy charged particles are generated due to strong laser-plasma interactions, which attracted many scientists not only by its fundamental physics but also by its wide applications toward nuclear physics and medical therapy.

Proton beams from laser-plasma interactions have attracted attentions due to its application to a cancer therapy system since the first demonstration of $60 \mathrm{MeV}$ proton beam with a large laser facility [1]. However, despite of many efforts and promising new acceleration mechanisms such as radiation pressure acceleration (RPA) [2] and collisonless shock acceleration (CSA) [3], its realization is still far reach. Recently, KAERI has proposed a new target structure named as Ion-Layer Embedded Foil (ILEF) target [4], which utilizes bulk electrostatic field instead of surface sheath field. A two-dimensional particle-in-cell (PIC) simulation showed that it could generate a high-energy proton beam with a narrow energy spread. Preliminary experimental investigations with a real-time detection system will be presented.

KAERI has also interested in the nuclear physics by using a laser-accelerated electron beam. An electron beam with energy less than $60 \mathrm{MeV}$ was bombarded on a $2 \mathrm{~mm}$-thick Ta converter to generate gamma-rays, which activated ${ }^{197} \mathrm{Au}(\gamma, \mathrm{n}){ }^{196} \mathrm{Au}$ reaction [5]. With a calibrated detector, the gamma-ray was estimated to be $6 \times 10^{17} \mathrm{ph} / \mathrm{cm}^{2}$ in photon flux and energy higher than $10 \mathrm{MeV}$ at $50 \mathrm{~cm}$ away from the Ta converted.

1. R. A. Snavely, et al., "Intense High-Energy Proton Beams from Petawatt-Laser Irradiation of Solids”, Phys. Rev. Lett. 85, 2945 (2000).

2. X. Q. Yan, et. al, "Generating High-Current Monoenergetic Proton Beams by a Circularly Polarized Laser Pulse in the Phase-Stable Acceleration Regime", Phys. Rev. Lett. 100, $135003(2008)$

3. L. O. Silva, et. al., "Proton Shock Acceleration in Laser-Plasma Interactions", Phys. Rev. Lett. 92, 015002 (2004)

4. K. N. Kim, et. al., "Quasi-monoenergetic proton beam from a proton-layer embedded metal foil irradiated by an intense laser pulse", Phys. Plasmas 23, 033119 (2016)

5. S. H. Park, et. al., "Gamma-ray generation using a laser-accelerated electron beam", SPIE Newsroom 003737 (2011) 


\section{Detection and optimization methods for plasma interactions and nuclear processes}

John Nees ${ }^{1}$, Zhao-Han He ${ }^{1,3}$, Jungmoo Hah ${ }^{1}$, Alexander Englesbe ${ }^{1}$, Keegan Behm ${ }^{1}$, Alec Thomas $^{1,2}$, Karl Krushelnick ${ }^{1}$

${ }^{1}$ Center for Ultrafast Optical Science, University of Michigan, Ann Arbor Michigan, USA nees@umich.eduJohn Nees, zhe@umich.edu Zhao-Han He,jmhah@mich.eduJungmoo Hah, aengles@umich.eduAlexanderEnglesbe,agrt@umich.eduAlecThomas,kmkr@umich.edu Karl Krushelnick

${ }^{2}$ University of Lancaster Lancaster,Lancashire,UK, alec.thomas@lancaster.ac.uk ${ }^{3}$ now at Cymer, San Diego, California, USA

Focused laser pulses are capable of driving the acceleration of electrons and other species with much higher field gradients than conventional accelerators. However, the results depend on critical parameters of the laser and of plasma environment. We discuss static and active control of spatial optical phase as a means of controlling acceleration and other properties in underdense and overdense plasmas. We also give examples of short-term and long-term temporal controls. Finally, we will discuss the significance of these methods on high-energy photon experiments.

1. T. Z. Zhao, K. Behm, Z-H. He, A. Maksimchuk, J. A. Nees, V. Yanovsky, A. G. R. Thomas, K. Krushelnick, "Characterization of electrons and X-rays produced using chirped laser pulses in a laser wakefield accelerator," Plasma Physics and Controlled Fusion, 58, 105003 (2016).

2. Z.-H. He, J.A. Nees, B. Hou, K. Krushelnick, A.G.R. Thomas, "Enhancement of plasma wakefield generation and self-compression of femtosecond laser pulses by ionization gradients, " Plasma Physics and Controlled Fusion, 56, 084010 (2014).

3. Z-H He, B. Hou, J. A. Nees, J. H. Easter, J. Faure, K. Krushelnick, and A. G. R. Thomas, "High repetition-rate wakefield electron source generated by few-millijoule, 30 fs laser pulses on a density downramp," New Journal of Physics, 15, 053016 (2013).

4. C. Zulick, B. Hou, F. Dollar, A. Maksimchuk, J. Nees, A. G. R. Thomas, Z. Zhao and K. Krushelnick, "High resolution bremsstrahlung and fast electron characterization in ultrafast intense laser-solid interactions," New Journal of Physics, 15, 123038 (2013). 


\title{
Nuclear Isomer Gamma Spectroscopy as a Tool for On-line Characterization of High Power Laser Generated Radiation
}

\author{
F. Negoita ${ }^{1,2}$, M. Gugiu ${ }^{1,2}$, C. Petrone ${ }^{2}$, D. Balabanski ${ }^{1}$, S. Balascuta ${ }^{1}$, C. Manailescu ${ }^{2}$,

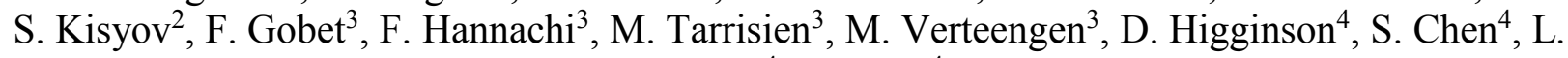 \\ Vassura $^{4}$, J. Fuchs ${ }^{4}$ \\ ${ }^{1}$ ELI-NP, IFIN-HH, 077125 Bucharest-Magurele, Romania, florin.negoita@eli-np.ro \\ ${ }^{2}$ DFN, IFIN-HH, 077125 Bucharest-Magurele, Romania \\ ${ }^{3}$ CENBG, CNRS-IN2P3 Université de Bordeaux, 33175 Gradignan, France \\ ${ }^{4}$ LULI, UMR 7605 CNRS-CEA-Ecole Polytechnique-Universite Paris VI, 91129 Palaiseau,
} France

We report the 'in-situ' measurement of the gamma ray spectrum from the decay of several nuclear isomers populated in reactions induced in a $\mathrm{Zr}$ target by laser accelerated protons with clear identification of the $6.2 \mathrm{~ms}$ halflife isomer in ${ }^{90} \mathrm{Nb}$. Enough high statistics $(\sim 100$ counts) was recorded for a single laser pulse demonstrating the possibility to apply gamma spectroscopy techniques for on-line, shot-by-shot, characterization of high power laser generated radiation at repetition rates as high as $10 \mathrm{~Hz}$. The capability to identify several isomeric states decaying in the same time through their specific gamma rays, allow to take advance of different thresholds in their production cross section and extract proton spectral information, extending the method proposed in [1].

The experiment [2] was performed at ELFIE facility at LULI (Palaiseau, France) using a $\mathrm{LaBr}_{3}$ :(Ce) scintillating crystal coupled to a photomultiplier and placed at about $\sim 15 \mathrm{~cm}$ from both primary (proton acceleration) and secondary (proton reaction) targets. The gamma spectrum obtained for a typical laser shot is shown in the figure below. A gate on the gamma arrival time relative to laser pulse impact on target is producing the spectrum in the inset where the $257 \mathrm{keV}$ gamma from $T_{1 / 2}=6.2 \mathrm{~ms}$ isomer decay is observed in negligible background conditions. The $122.4 \mathrm{keV}$ line originates from $\mathrm{T}_{1 / 2}=18.8 \mathrm{~s}$ isomer populated in the ${ }^{90} \mathrm{Zr}(\mathrm{p}, \mathrm{n}){ }^{90} \mathrm{Nb}^{*}$ reaction, while $206 \mathrm{keV}$ line corresponds to $5.1 \mathrm{~s}$ isomer in ${ }^{79} \mathrm{Br}$ produced through $\left(\gamma, \gamma^{\prime}\right)$ photonuclear reaction in the scintillator itself.

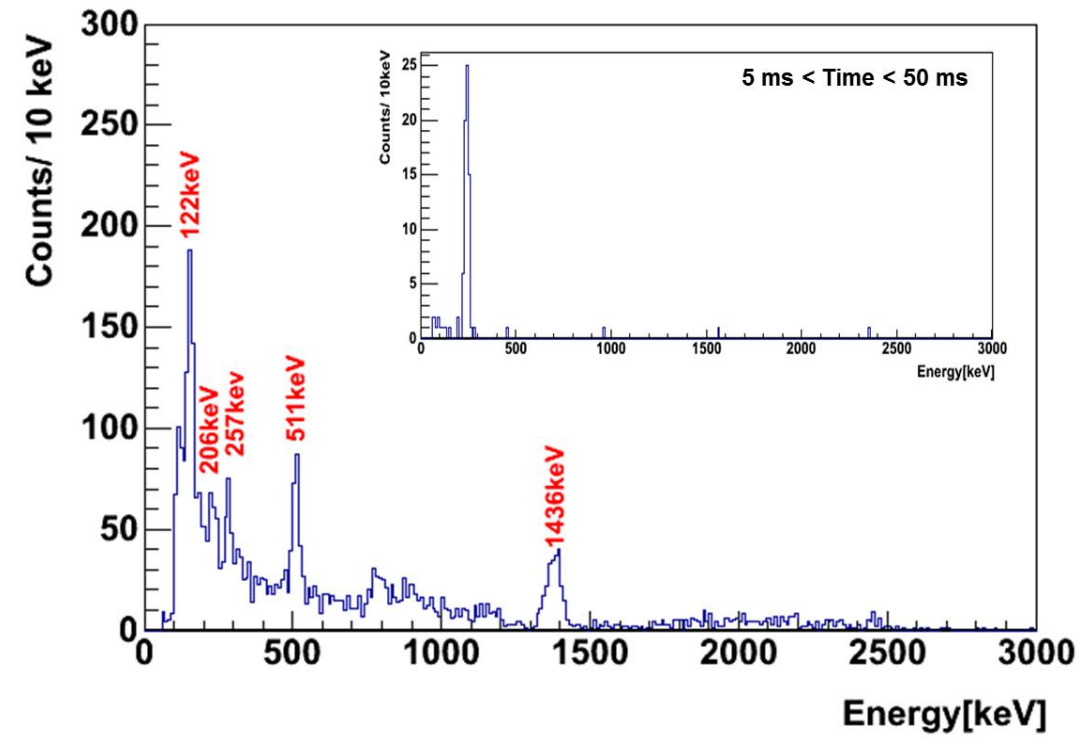


The proton energy spectrum measured with RCF stack was convoluted with isomer production cross sections predicted by Talys code [3] for mentioned $(p, n)$ reaction. The number of expected counts thus calculated and found in good agreement with measured gamma yields.

The proposed diagnostic method has several advantages:

- it is applicable for protons and (heavy) ions simultaneously

- it can be used for prompt high energy gamma rays spectral measurement, similar to activation method described in [4] but applied for nuclear isomeric states

- it has a large angular acceptance complementing the techniques based on magnetic spectrometers (Thomson parabola)

- it can be used in transmission (using foils) with rather low impact on traversing radiation

As a case study, the results of simulations in several configurations of interest for characterization of laser accelerated proton at energies required by cancer therapy (up to 250 $\mathrm{MeV}$ ) will be presented and some anticipated difficulties will be discussed.

The method is dependent on the cross section for production of isomeric states. Though predicted by nuclear reaction models, there is limited experimental data on such cross sections. A situation that can be turn into an opportunity for laser driven experiments to measure this type of nuclear data and contribute to models improvement.

1. R.J. Clark et al., "Detection of short lived radioisotopes as a fast diagnostic for intense lasersolid interactions", Applied Physics Letters, 89, 141117 (2006).

2. F. Negoita et al., "Perspectives for neutron and gamma spectroscopy in high power laser driven experiments at ELI-NP", Exotic Nuclei and Nuclear/Particle Astrophysics (V). From Nuclei to Stars, Proceedings of the Carpathian Summer School of Physics, Sinaia, Romania, July 13-26, 2014, AIP Conference Proceedings 1645 (2015) 228-236, Edited by: L. Trache, D. Chesneanu, CA Ur.

3. A.J. Koning, S. Hilaire and M.C. Duijvestijn, "TALYS-1.0", Proceedings of the International Conference on Nuclear Data for Science and Technology, April 22-27, 2007, Nice, France, editors O.Bersillon, F.Gunsing, E.Bauge, R.Jacqmin, and S.Leray, EDP Sciences, 2008, p. 211-214.

4. M. Roth, "Diagnostics for ultra-short pulse laser-produced plasma", Journal of Instrumentation, 6, R09001 (2011). 


\title{
Application-enabling High Repetition PW Lasers: HAPLS Overview and Scaling
}

\author{
Craig W. Siders and Constantin Haefner \\ Advanced Photon Technologies, NIF \& Photon Science Directorate, \\ Lawrence Livermore National Laboratory, Livermore, CA, USA \\ Siders2@llnl.gov,Haefner2@llnl.gov
}

Worldwide, several national-scale, large short pulse laser projects are currently underway, pushing the limits of laser technology. LLNL is building the diode pumped $10 \mathrm{~Hz} \mathrm{High-}$ repetition-rate Advanced Petawatt Laser System (HAPLS) for the Extreme Light Infrastructure. By "closing the loop" from output diagnostics and experiment results, rep-rated high peak power lasers using will allow closing the "facility gap" of peak power produced to intensity on target delivered: adaptive loops enable performance tuning to achieve ultrahigh intensities and maintain those from shot to shot. These next generation scientific lasers are enabled by the recent advancement of optical materials and processing techniques, high performance computers for laser performance modeling and control systems, diode technology and new laser architectures. One of the most remarkable and promising opportunities of these light sources is the combination of high peak intensity, high energy, short pulses, and high repetition rate allowing high-field experiments, including nuclear photonics, of unprecedented fidelity and commercial applications enabled by these capabilities. 


\section{Friday, October 21, 2016 \\ Gamma Source Technologies \& Applications: 10:50 13:00 Session Chair: J. Ness}




\section{Advances in Scintillator Detector Materials}

S. Payne, N. Cherepy, N. Zaitseva, P. Beck, E. Swanberg, Z. Seeley, P. Martinez, L. Carman, A. Mabe, A. Glenn, S. Hunter, R. Sanner, B. Wihl, I. Jones, N Harvey

\section{Lawrence Livermore Laboratory, Livermore, CA USA; payne3@llnl.gov}

Herein we summarize our discoveries and development of numerous of new scintillator detectors, including:

- Inorganics: $\mathrm{SrI}_{2}(\mathrm{Eu})$ single crystals, offering high $(3.0 \%)$ resolution, same as $\mathrm{LaBr}_{3}(\mathrm{Ce})$

- Transparent ceramic garnets: GYGAG(Ce), first high-resolution oxide

- Gamma-detecting plastics: Organo-bismuth-loaded polymers, exhibiting first prominent photo-peak in a plastic scintillator

- High-energy neutron detecting plastic: PPO-loaded polystyrene, with gamma pulse-shape discrimination (PSD)

- Thermal neutron detecting plastics: Lithium and boron-loaded polymers with PSD, capable of distinguishing thermal and high-energy neutrons

- Organic crystals: Inexpensive solution-grown stilbene, best high-energy neutron detector

In addition, new aspects of scintillator physics will be discussed, as will the development of state-of-the-art instruments based on these materials.

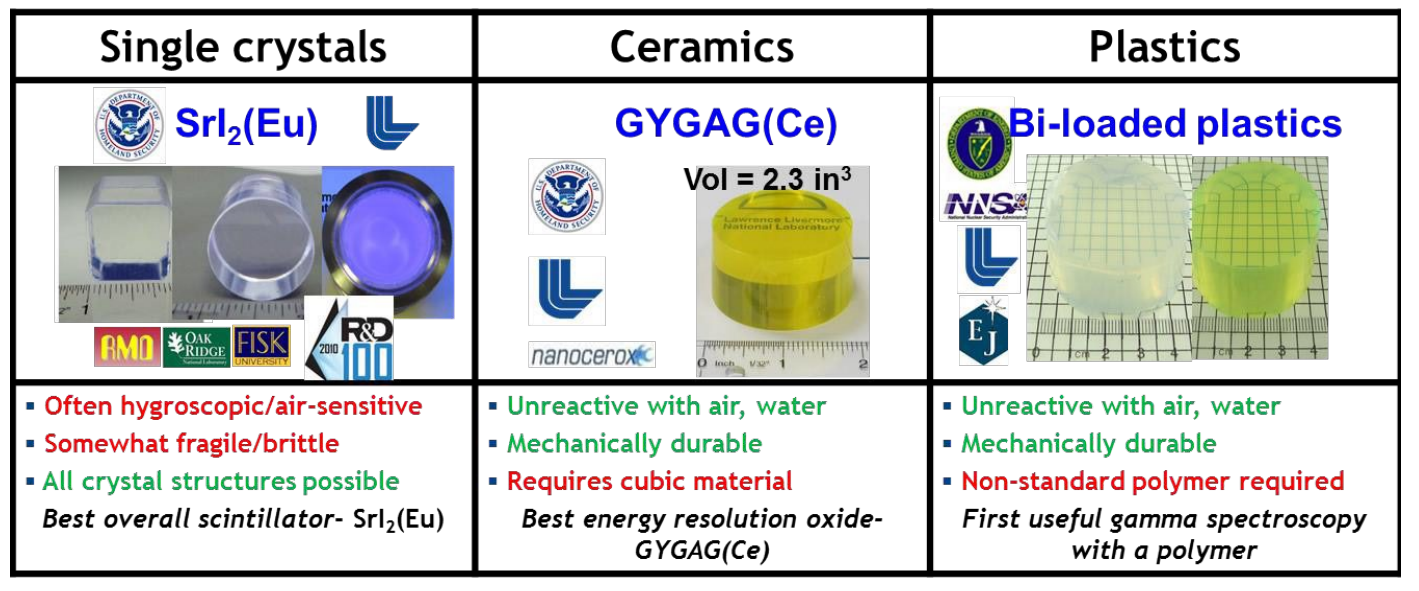
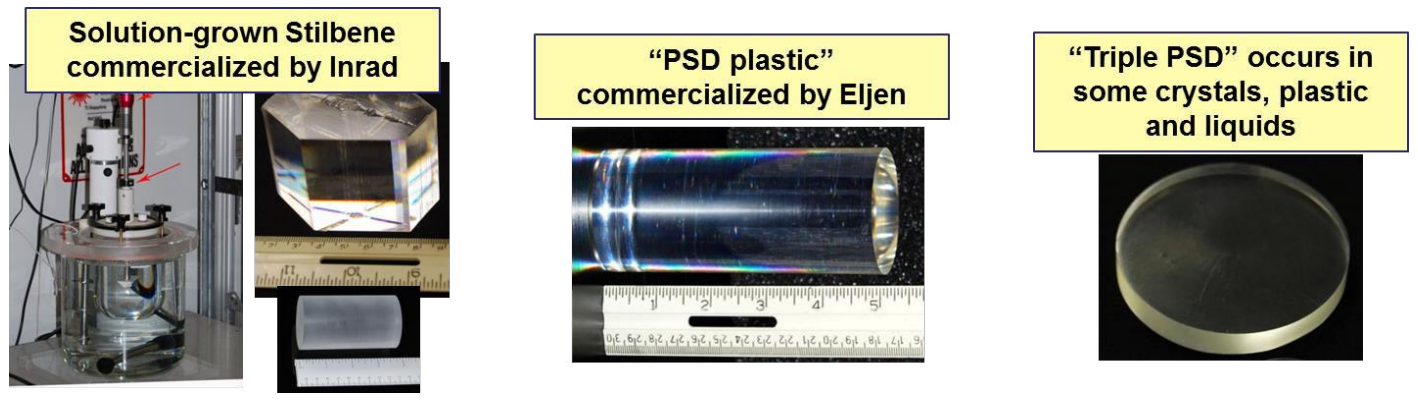

This work was supported by the National Nuclear Security Administration, Defense Nuclear Nonproliferation Research and Development Office, and was performed under the auspices of the Department of Energy by Lawrence Livermore National Laboratory under Contract DE-AC52-07NA27344. This work was also supported by the Domestic Nuclear Detection Office of DHS and J9NTD of DTRA. This support does not constitute an express or implied endorsement on the part of the Government. 


\section{Development of High Repetition Rate Inverse Compton Scattering Gamma Source with Laser Pulse Recirculation}

Alex Murokh, RadiaBeam Technologies LLC

Accelerator driven Inverse Compton Scattering (ICS) sources has been considered for decades as compact alternatives to the synchrotron light sources. In ICS a high brightness relativistic electron beam interacts with the counter-propagating intense laser pulse, generating a beam of back-scattered photons, which are Doppler-shifted into the X-ray and gamma spectral range. Although the physics of ICS interaction is straight forward, its practical implementation is challenging due to the need for a precision manipulation and synchronization of the intense picosecond laser and electron beams, as well as recirculating laser power through maintaining the interaction over the long pulse trains. In this paper, we report on the recent progress in ICS technology development, discuss ICS applications, compare ICS to alternative X-ray sources, and discuss future development plans. 


\title{
Enhancement Cavity-based Laser-Compton Scattering Photon Source
}

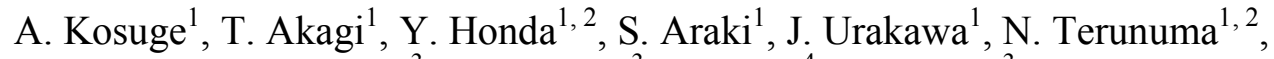

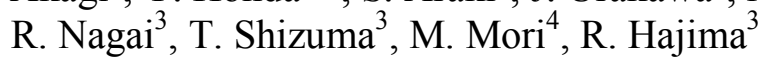 \\ ${ }^{1}$ High Energy Accelerator Research Organization (KEK), Oho, Tsukuba, Ibaraki, 305-0801, \\ Japan \\ atsushi.kosuge@kek.jp,akagit@post.kek.jp,yosuke@post.kek.jp,sakae.araki@kek.jp, \\ junji.urakawa@kek.jp,nobuhiro.terunuma@kek.jp \\ ${ }^{2}$ Science of the Graduate University for Advanced Studies (SOKENDAI) \\ ${ }^{3}$ National Institutes for Quantum and Radiological Science and Technology (QST), Tokai, Naka, \\ Ibaraki 319-1106, Japan \\ ${ }^{4}$ Kansai Photon Science Institute, National Institutes of Quantum and Radiological Science and \\ Technology, 8-1-7 Umemidai, Kizugawa 619-0215, Japan \\ nagai.ryoji@qst.go.jp,shizuma.toshiyuki@qst.go.jp,mori.michiaki@qst.go.jp, \\ hajima.ryoichi@qst.go.jp
}

Nowadays, generation of narrow-bandwidth, high brightness and energy tunable photons via Laser-Compton scattering (LCS) attracts attention for many scientific and industrial applications. The LCS photons are generated by collision of laser photons and relativistic electrons. Traditional methods for LCS experiment employ low repetition rate laser system [1]. Due to the small cross-section of LCS, although the low repetition rate lasers can produce much higher pulse energies, they consequently have a limited photon flux. To overcome this, a resonantly enhanced optical pulse inside a cavity, namely enhancement cavity, is employed. Laser pulses from a mode-locked laser oscillator (repetition rate of tens to hundreds of megahertz) are added coherently inside the cavity, the intracavity power increases by orders of magnitude. To enhance an optical pulse inside a cavity, the repetition frequency of the enhancement cavity and the seeding laser must be locked actively to maintain the resonance condition. Various schemas have been developed to lock the cavity such as the Pound-Drever-Hall method, the tilt-locking method and the Hänsch-Couillaud method [2, 3]. This enhancement cavity has received broad attention in the laser technology for the purpose of high harmonic generation (HHG) because of HHG inside a cavity with a multimegahertz repetition rate. Recently, stable cavity operation with a power enhancement of around 1300 for average power of $670 \mathrm{~kW}$ with $10 \mathrm{ps}$ pulses at a repetition rate of $250 \mathrm{MHz}$ has been reported [4]. A recent work using an enhancement cavity scheme has led to harmonic generation reaching photon energy of $100 \mathrm{eV}$ [5]. In the future, circulating powers scaling to MW range are pursued. However, there are some limitations to increase intracavity power; mirror damage and thermal effects on the mirror. To mitigate these problems, a cavity with a large spot size on the mirrors is proposed [6]. This high intracavity power and high repetition rate are the advantages of the enhancement cavity to realize a highflux high-repetition LCS source.

We developed a laser enhancement cavity and installed the cavity at an energy recovery linac, the Compact ERL (cERL), to demonstrated LCS photon generation. The experimental setup is depicted in Fig. 1 (a). A seed laser system delivers an average power of $45 \mathrm{~W}$ and pulse duration of $10 \mathrm{ps}$ (FWHM) to the enhancement cavity. The pulse repetition rate is $\mathrm{f}_{\text {rep }}=162.5 \mathrm{MHz}$ which has an integer relation with the repetition rate of the electron beam. The laser beam is injected to a four-mirror enhancement cavity with two concave mirrors to produce a small spot laser beam inside a cavity. In the enhancement cavity-based LCS source, we need to synchronize three 
(a)

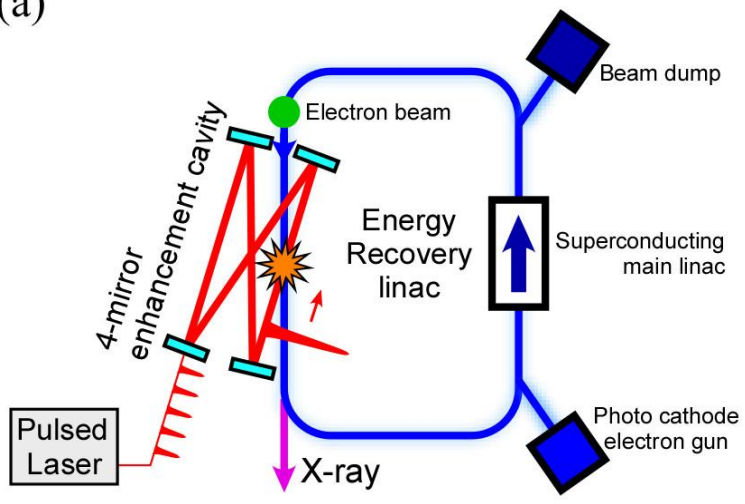

(b)

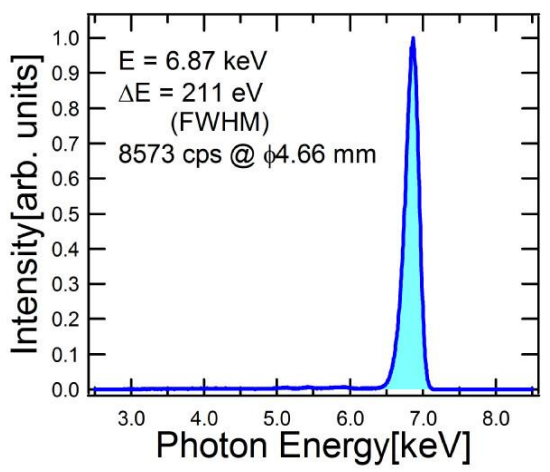

Figure 1 (a) Schematic drawing of the LCS experimental setup. (b) Measured LCS spectrum.

systems: the seed laser cavity, the enhancement cavity and the accelerator. This synchronization is more complicate in comparison with HHG experiments. We developed such synchronization system with a phase locked loop method and achieved a maximum circulating power of $10.4 \mathrm{~kW}$ with an injection power of $24 \mathrm{~W}$.

The cERL is an energy recovery linac based on a superconducting accelerator in KEK to demonstrate both low-emittance and high average current operation. The electron beam was accelerated to $20 \mathrm{MeV}$ and transported to the collision point. Recently, we have achieved to circulate $1 \mathrm{~mA}$ at $162.5 \mathrm{MHz}$.

The LCS photons travel through an evacuated beam pipe with beryllium windows to the experimental hatch. Around $7 \mathrm{keV}$ X-ray was generated by collision of $1064 \mathrm{~nm}$ laser photons and $20 \mathrm{MeV}$ electrons at an angle of $18^{\circ}$ and measured by a silicon drift detector (SDD: XR100SDD, AMPTEK Inc.). From the measured SDD spectrum, the peak energy of $6.87 \mathrm{keV}$, the FWHM spectrum width of $211 \mathrm{eV}$ and detector count rate of $8573 \mathrm{cps}$ was obtained within a detector area, $\phi 4: 66 \mathrm{~mm}$ as shown in Fig. 1 (b). The measured spectrum width reflects the detector resolution. When we take into account the detector resolution, we estimate the actual spectrum width to be $118 \mathrm{eV}$. The LCS photon flux at the collision point is estimated to be $1.6 \times$ $10^{8}$ photons/sec from CAIN/EGS simulation [7] with an electron beam current of $0.9 \mathrm{~mA}$ and an average laser power of $6.4 \mathrm{~kW}$.

This work was supported by Photon and Quantum Basic Research Coordinated Development Program from the Ministry of Education, Culture, Sports, Science and Technology, Japan.

1. C. Bemporad et al., "High-Energy Photons from Compton Scattering of Light on 6.0-GeV Electrons" Phys. Rev. 138, B1546 (1965)

2. T. W. Hansch and B. Coulliard, "Laser frequency stabilization by polarization spectroscopy of a reflecting reference cavity" Opt. Commun. 35 441(1980).

3. A. Kosuge et al., "Polarization-selectable cavity locking method for generation of laser Compton scattered $\gamma$-rays" Opt. Express 22, 6613 (2014)

4. H. Carstens et al., "Megawatt-scale average-power ultrashort pulses in an enhancement cavity" Opt. Lett. 39, 2595 (2014)

5. H. Carstens et al., "High-harmonic generation at $250 \mathrm{MHz}$ with photon energies exceeding 100 eV" Optica 3, 366 (2016)

6. H. Carstens et al., " Large-mode enhancement cavities" Opt. Express 21, 11606 (2013).

7. P.Chen et al., "CAIN: Conglomérat d'ABEL et d'Interactions Non-linéaires" Nucl. Instr. Meth. A 355, 107 (1995). 


\title{
Developments of optical system for $\mathrm{X} /$ gamma ray Compton machines
}

\author{
G. Cagnoli ${ }^{1}$, E. Cormier ${ }^{2}$, T. Omori ${ }^{3}$, K. Sakaue ${ }^{4}$, T. Takahashi ${ }^{5}$, N. Terunuma ${ }^{3}$, \\ J. Urakawa ${ }^{3}$, A. Variola ${ }^{6}$, F. Zomer ${ }^{7}$ \\ ${ }^{1}$ LMA, CNRS/IN2P3, 7 Avenue Pierre de Coubertin, 69622 Villeurbanne Cedex, France, \\ g.cagnoli@lma.in2p3.fr \\ ${ }^{2}$ CELIA, Université Bordeaux, CNRS, 351 Cours de la libération, F-33405 Talence, France, \\ eric.cormier@celia.u-bordeauxl.fr \\ ${ }^{3}$ High Energy Accelerator Research Organization (KEK), 1-1 Oho, Tsukuba, Ibaraki 305-0801, \\ Japan,tsunehiko.omori@kek.jp,nobuhiro.terunuma@kek.jp, urakawa@post.kek.jp, \\ ${ }^{4}$ Waseda Institute for Advanced Study (WIAS), Waseda University, 3-4-1 Okubo, Shinjuku-ku, \\ Tokyo 169-8555, Japan, \\ kazuyuki.sakaue@aoni.waseda.jp \\ ${ }^{5}$ Graduate School of Advanced Sciences of Matter (AdSM), Hiroshima University, 1-3-1 \\ Kagamiyama, Higashi Hiroshima, Hiroshima 739-8530, Japan, \\ tohru-takahashi@hiroshima-u.ac.jp \\ ${ }^{6}$ LNF-INFN, Via E. Fermi 40, 00044 Frascati, Italy \\ , alessandro.variola@lnf.infn.it \\ ${ }^{7}$ LAL, Université Paris-Sud, CNRS/IN2P3, 91400 Orsay, France, \\ zomer@lal.in2p3.fr
}

Production of $\mathrm{X}$ and gamma rays via laser-electron Compton scattering is still a challenging technology. To reach high $\mathrm{X}$ or gamma ray flux as required by many applications (i.e. radiotherapy, $R \& D$ and nuclear excitations) the use of high finesse optical resonators has been proposed some time ago [1,2]. Since then, with the advance of Yb-dopped fiber laser amplification high seed laser average power become available [3]. Advanced optical resonator designs dedicated to laser-electron scattering also appeared. These key elements of Compton machines have been tested on electron accelerators and are still under development.

A joint Japanese-French Collaboration (Hiroshima Univ., KEK, Waseda Univ. and CELIA, LAL, LMA Laboratories) have been set up to perform a R\&D on optical cavities and X/gamma ray machine designs. An overview of the obtained results will be given in this presentation. This will cover the optical cavity developments; the results obtained on electrons accelerators (ATF, cERL , LUCX ); present developments on cavity burst mode [4] dedicated to compact LINACs; novel laser-cavity scheme under development at KEK. Perspective and expected performances will be given finally.

This presentation will be given on the behalf of a Japanese-French Collaboration (Hiroshima Univ., KEK, Waseda Univ. and CELIA, LAL, LMA Laboratories).

1. L. Federici et al., Il Nuovo. Cim. B59 (1980) 247.

2. Z. Huang and R.D Ruth, Phys. Rev. Lett. 80 (1998) 976.

3. H. Carstens et al., Opt. Lett. 39 (2014) 2595.

4. K. Sakaue et al., Nucl. Instr. Meth. A637 (2011) 5107. 


\title{
Neutron Angular Distribution in $(\gamma, \mathbf{n})$ Reactions with Linearly Polarized $\gamma$-ray Beams
}

\author{
Takehito Hayakawa ${ }^{1}$ \\ ${ }^{2}$ National Institute for Quantum and Radiological Science and Technology, \\ Tokai, Ibaraki, Japan \\ hayakawa.takehito@qst.go.jp
}

The $(\gamma, \mathrm{n})$ reactions with laser Compton scattering (LCS) $\gamma$ ray beam are useful tools for the study of nuclear physics and various applications. It is possible to create almost $100 \%$ linear polarized LCS $\gamma$-ray beam because the polarization of the incident laser is directly transported to the scattered $\gamma$-ray beam. The M1 strength from the ground state (or level density of $1+$ states) is of importance for estimation of the interaction strength between a neutrino and a nucleus for the study of supernovae [1]. However, it is difficult to measure M1 strength in the energy region of giant dipole resonance (GDR) because of its large E1 strength. Thus, we have investigated a new method to measure the M1 strength by measuring the spatial anisotropy of neutrons emitted from $(\gamma, \mathrm{n})$ reactions with linear polarized LCS $\gamma$-ray beam. In 1957, Agodi [2] predicted theoretically angular distribution of neutrons emitted from states excited via dipole transitions (E1 or M1) with linearly polarized $\gamma$-rays at the polar angle of $\theta=90^{\circ}$ should be followed by a simple function, $\mathrm{a}+\mathrm{b} \cos (2 \phi)$, where $\phi$ is azimuthal angel, and that the sign of $\mathrm{b}$ in this function for M1 transitions is different from that of E1 transitions. However, this theoretical prediction has not been verified over the wide mass region except for light nuclei. We measured neutron angular distributions with $(\gamma, \mathrm{n})$ reactions on $\mathrm{Au}, \mathrm{I}$, and ${ }^{\text {nat }} \mathrm{Cu}$ using linear polarized LCS $\gamma$-ray beams at NewSUBARU [3]. The neutron energy was measured using a time-of-flight method with a plastic scintillator. The neutron yields were measured as a function of the angle of linear polarization plane. The neutron yields are well reproduced by kai-square fitting with the function of $a+b \cos (2 \phi)$ [3]. We verified the Agodi's prediction for the first time over the wide mass region. To study the detailed spatial anisotropy, we also measured neutron yields from (polarized $\gamma, \mathrm{n}$ ) reactions on a ${ }^{\text {nat }} \mathrm{Fe}$ target [4]. This neutron yields was also described well by the Agodi's function.

Another feature of LCS beams is the sharp energy spread which is useful for a newly proposed selective isotope transmutation of long-lived fission products (LLFPs) including ${ }^{93} \mathrm{Zr}$ and ${ }^{107} \mathrm{Pd}$ [5]. In this method, when an element target including a LLFP is irritated by a suitable LCS beam, the only LLFP is selectively transmuted into stable or short-lived unstable isotopes.

1. M. Cheoun, et al., "Neutrino reactions on ${ }^{138} \mathrm{La}$ and ${ }^{180} \mathrm{Ta}$ via charged and neutral currents by the quasiparticle random-phase approximation," Phys. Rev. C 82, 03504 (2010).

2. A. Agodi, "On $\gamma$-polarization effects in photonuclear reactions," Il Nuovo Cimento, 5(1), 21 (1957).

3. K. Horikawa, et al., "Neutron angular distribution in $(\gamma, \mathrm{n})$ reactions with linearly polarized $\gamma$-ray beam generated by laser Compton scattering," Phys. Lett. B, 737, 109 (2014).

4. T. Hayakawa, et al., "Spatial anisotropy of neutrons emitted from the ${ }^{56} \mathrm{Fe}(\gamma, n){ }^{55} \mathrm{Fe}$ reaction with a linearly polarized $\gamma$-ray beam," Phys. Rev. C 93, 044313 (2016)

5. T. Hayakawa et al., "Proposal for selective isotope transmutation of long-lived fission products using quasi-monochromatic $\gamma$-ray beams," J. Nucl. Sci. Tech. in press (2016), http://dx.doi.org/10.1080/00223131.2016.1194776 


\section{Page Intentionally Left Blank}

Proc. of SPIE Vol. 10419 1041901-144

Downloaded From: https://www.spiedigitallibrary.org/conference-proceedings-of-spie on 26 Apr 2023 Terms of Use: https://www.spiedigitallibrary.org/terms-of-use 


\section{Friday, October 21, 2016 \\ Closing Remarks and Announcement Regarding Nuclear Photonics 2018}




\section{Page Intentionally Left Blank}

Proc. of SPIE Vol. $104191041901-146$

Downloaded From: https://www.spiedigitallibrary.org/conference-proceedings-of-spie on 26 Apr 2023 Terms of Use: https://www.spiedigitallibrary.org/terms-of-use 


\section{Tuesday, October 18, 2016 \\ Evening Poster Session and Networking: 19:00 22:00}




\title{
The Introduction of Development Project for Laser Driven Compact Neutron Source in Hamamatsu
}

\author{
Ryohei Hanayama $^{1}$, Haruyasu Kondo ${ }^{1}$, Atsushi Sunahara ${ }^{2}$, Hiroki Tanaka ${ }^{3}$, \\ Shunsuke Kurosawa ${ }^{4}$, Kunioki Mima ${ }^{1}$, Yoshiaki Kato ${ }^{1}$ \\ ${ }^{1}$ The Graduate School for the Creation of New Photonics Industries, Hamamatsu, Japan, \\ hanayama@gpi.ac.jp,haruyasu@gpi.ac.jp,k.mima@gpi.ac.jp,y.kato@gpi.ac.jp, \\ ${ }^{2}$ Institute for Laser Technology,Osaka,Japan,suna@ilt.or.jp, \\ ${ }^{3}$ Kyoto University, Kumatori, Japan, h-tanaka@rri.kyoto-u.ac.jp, \\ ${ }^{4}$ Tohoku University, Sendai, Japan, kurosawa@imr.tohoku.ac.jp
}

We introduce the research project about the development of laser driven compact neutron source in this report.

It is needless to say, neutron science is one of the key technologies for near future. It can be used for nondestructive inspection, homeland security, material modification, medical therapy and so on. However, these technologies are still in scientific research state. And the industrial market for neutron technologies is still small or not opened. Especially in Japan, after the huge earthquake in 2011, All of nuclear reactors were shutdown. Some of those were important neutron sources. Therefore, it is eager for emergence of alternative neutron sources. In such a situation, the compact accelerator driven neutron source "RANS" which was constructed by RIKEN in 2013[1] drew the attention of industrial region in Japan. And in this year, Japan Science and Technology Agency started new program, which is titled "Development of key technologies for compact neutron source and its industrial application", in their competitive funding program, "A-STEP". The project introduced in this paper is one of the project of the program.

The key features of our project are laser driven neutron, repetitive neutron generation, directional emission and approaching for new application area using fast neutrons. In our project, the neutron generation scheme, such as shown in Fig. 1, is assumed. In this scheme, laser driven ion beam is emitted from the first target, named "pitcher". The ion beam induces nuclear reaction at the second target, named "catcher" [2]. We assume DD beam fusion, Li-P reaction or so, as the nuclear reaction. We can control the spectrum of generated neutron beam by selecting nuclides and energy. Moreover, directional neutron generation can be realized in this scheme [3]. The directional neutron generation has the advantage for the reduction of shielding materials and the total weight of the whole system. We use the diode pumped ultra-intense laser of Hamamatsu photonics, as the driver. Both of the laboratory of Hamamatsu photonics and GPI, which is core institute of this project, are located in Hamamatsu city. Therefore, we call our project "Hamamatsu laser driven neutron source".

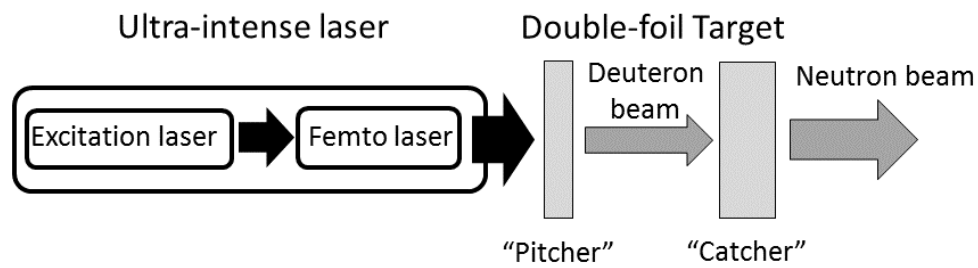

Fig.1 The schematics of the laser driven compact neutron source 


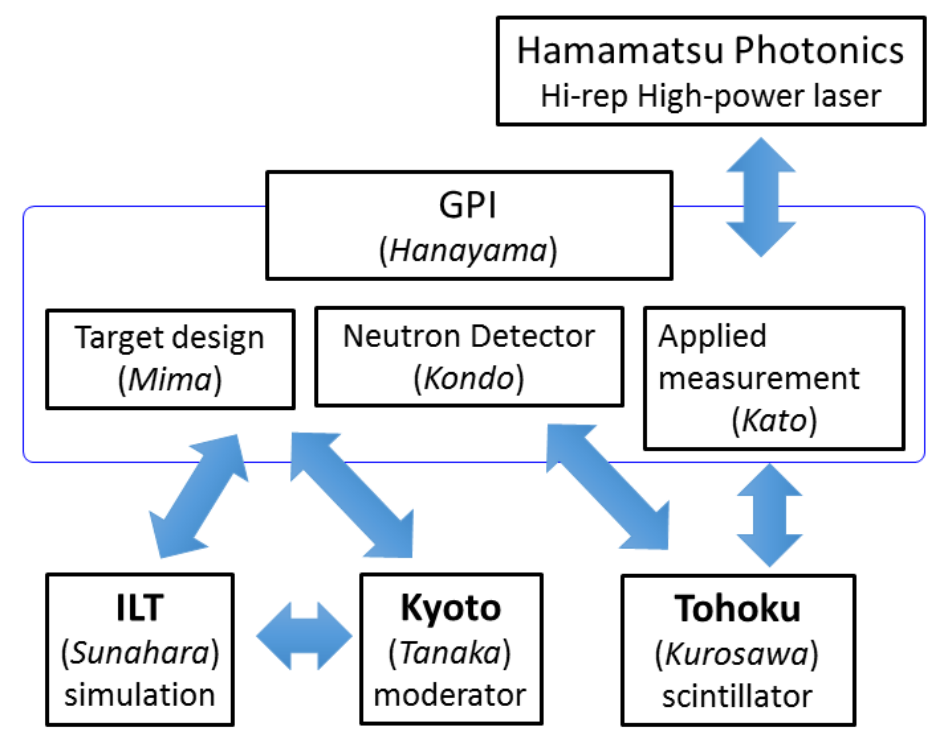

Fig.2 The team organization and roles in our project

Fig. 2 shows the organization of our team. It consists of four universities or research institute and one cooperative private company. GPI totalize the team. ILT and Kyoto university mainly investigate the target design from theoretical side. Tohoku university is developing new scintillation material for fast neutron detection. One of the most challenging task of our project is to construct the whole system as a stable repetitive neutron source. Constricting continuous fuelpellet supplying is also included the great challenge. We are studying and discussing about the continuous fuel-pellet supplying. Any suggestions are welcome. The duration of continuous neutron emission is important parameter for the design of the fuel-pellet supplying system. In other words, there will be a big branch for that we will create quasi-infinite system or finitesystem. We are discussing about this branch from the industrial and practical point of view, now. As far as its concerns to the control system, we have to make arrangement the pace of all of subsystems, such as fuel-pellet supplying, laser shooting, neutron monitoring and so on. We will use a real-time controller for totalize whole of system. These system constructing task are main task of totalization in GPI.

In the presentation, we will show some initial experimental results, in addition to the brief introduction of this project.

This project is supported by Adaptable and Seamless Technology Transfer Program through Target-driven R\&D (A-STEP) of Japan Science and Technology Agency. This project is being carried out with the great cooperation of the high-power laser division of Hamamatsu Photonics K. K. The authors express thanks to Dr. Kawashima and all researchers in the division.

1. http://rans.riken.jp/en/index.html

2. J.Alvarez, J.Fernadez, K.Mima, S.nakai. Y.Kato, et al., "Laser Driven Neutron Sources: Characteristics, Applications and Prospects," Physics procedia, 60, 29-38 (2014).

3. R,Roth, et al., "Bright laser driven neutron source based on the relativistic transparency of solid," Phys. Rev. Letters, 110 044802(2013). 


\title{
Aspects of quantum electrodynamics in laser-plasma and laser-particle interactions
}

\author{
Mattias Marklund, Arkady Gonoskov, Anton Ilderton \\ Department of Physics, Chalmers University of Technology, Gothenburg, Sweden \\ mattias.marklund@,chalmers.se, arkady.gonoskov@chalmers.se, anton.ilderton@.chalmers.se
}

Quantum electrodynamics (QED) is expected to play an increasingly significant role in intense laser-matter interactions. Some next-generation facilities, as well as upgrades of current ones, aims at reaching parameter domains where quantum aspects of light-matter interactions will be important.

In order to understand the QED aspects of current and future experiments, theory development is central. Here we review both some analytical as well as numerical developments in this field. In particular, common extensions of particle-in-cell (PIC) schemes [1] which account for strong field phenomena in laser-plasma interactions are described. We discuss the most important processes in these systems, taking into account the quantized nature of emission from charged particles, as well as various mechanisms of electron-positron pair production. We note that despite the low energy of photons in a laser, the approach is not limited to low-energy physics. Application of and problems with the current approaches, possible solutions to these problems, as well as possible applications will be discussed.

1. A. Gonoskov, S. Bastrakov, E. Efimenko, A. Ilderton, M. Marklund, I. Meyerov, A. Muraviev, A. Sergeev, I. Surmin, and E. Wallin, "Extended particle-in-cell schemes for physics in ultrastrong laser fields: Review and developments, "Physical Review E, 92, 023305 (2015). 


\title{
Nuclear reactions of astrophysical interest in laser plasmas
}

\author{
Yuanbin $\mathrm{Wu}^{1}$, Christoph H. Keitel ${ }^{1}$, Adriana Pálffy ${ }^{1}$ \\ ${ }^{1}$ Max-Planck-Institut für Kernphysik, Saupfercheckweg 1, D-69117 Heidelberg, Germany \\ yuanbin.wu@mpi-hd.mpg.de,keitel@mpi-hd.mpg.de,Palffy@mpi-hd.mpg.de
}

In astrophysical environments, matter is usually in the state of plasma. The properties of nuclear matter, such as reaction mechanisms and life times, may drastically differ in the plasma environment from the ones in conventional laboratory environments. In this context, the role of electron screening is one of the most crucial aspects. Determining the appropriate experimental conditions that allow to evaluate the nuclear reactions in stellar environments could strongly contribute to the development of nuclear astrophysics. The study of direct measurements of reactions in plasmas provides this chance [1].

Especially at the upcoming ELI-NP facility with 10 PW lasers, an experimental set-up where two laser beams generate two colliding plasmas is envisaged [1]. This will give the opportunity to investigate nuclear reactions under extreme plasma conditions. In this experimental set-up, a laser pulse interacting on a solid target produces a plasma through the Target Normal Sheath Acceleration (TNSA) mechanism, and then this rapidly streaming plasma interacts on a secondary plasma created by the interaction of a second laser pulse on a gas jet target [1]. We apply here an isothermal, fluid model [2,3] to describe the TNSA scheme in the laser-target interaction. With the ion spectrum obtained from this model, we study the nuclear reactions in the interaction of the rapidly streaming plasma on the secondary plasma.

As the first case of study, we analyze the reaction ${ }^{13} \mathrm{C}\left({ }^{4} \mathrm{He}, \mathrm{n}\right){ }^{16} \mathrm{O}$, which is one of the important helium burning processes as well as one of the main neutron sources for s-process. We obtain the neutron spectra and the total numbers of neutron events expected at this experimental set-up, based on the parameters of ELI-NP lasers. The results show the possibility of the detection of electron screening effects in stellar reactions. Extending the study to other astrophysical reactions, the experimental set-up will provide a strong tool to explore the nuclear reactions of astrophysical interest.

1. M. Roth et al. (eds.), "Laser driven nuclear physics at ELI-NP — technical design report," 2015.

2. P. Mora, "Plasma expansion into a vacuum," Phys. Rev. Lett. 90, 185002 (2003).

3. J. Fuchs et al., "Laser-driven proton scaling laws and new paths towards energy increase," Nature Phys. 2, 48 (2006). 


\section{QED-driven laser absorption}

M C Levy ${ }^{1}$, T G Blackburn ${ }^{2, *}$, N Ratan ${ }^{1}$, J Sadler ${ }^{1}$, C P Ridgers ${ }^{3}$, M Kasim¹, L Cuervorst $^{1}$, J Holloway ${ }^{1}$, M G Baring ${ }^{4}$, A R Bell ${ }^{1}$, S H Glenzer ${ }^{5}$, G Gregori ${ }^{1}$,

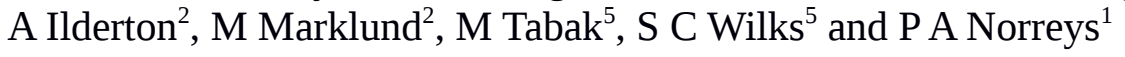

${ }^{1}$ Department of Physics, University of Oxford, Parks Road, Oxford OX1 3PU, UK matthew.levy@physics.ox.ac.uk,naren.ratan@physics.ox.ac.uk, james.sadler@physics.ox.ac.uk, muhammed.kasim@physics.ox.ac.uk,luke.cuervorst@physics.ox.ac.uk, james.holloway@physics.ox.ac.uk,tony.bell@physics.ox.ac.uk, gianluca.gregori@physics.ox.ac.uk,peter.norreys@physics.ox.ac.uk

${ }^{2}$ Department of Physics, Chalmers University of Technology, SE-41296 Gothenburg, Sweden tom.blackburn@chalmers.se, ilderton@chalmers.se, mattias.marklund@chalmers.se

${ }^{3}$ York Plasma Institute, University of York, York YO10 5DD, UK christopher.ridgers@york.ac.uk

${ }^{4}$ Department of Physics and Astronomy, Rice University, Houston, Texas 77005, USA baring@spacibm.rice.edu

${ }^{5}$ Lawrence Livermore National Laboratory, Livermore, California 94551, USA glenzer@slac.stanford.edu, tabak1@llnl.gov, wilks1@llnl.gov

Absorption describes the physical processes whereby the energy of an intense laser pulse is transferred to the particles of a supercritical plasma. Understanding it underpins important petawatt-scale applications, such as proton beam production for radiotherapy [1]. However, development of these applications has been hindered since no study thus far has described absorption throughout the transition from the classical to the quantum-electrodynamical (QED) regime of plasma physics [2,3]. We will present a model of absorption that holds over six orders of magnitude in laser intensity, self-consistently accounting for gamma-ray emission and electron-positron pair creation [4]. This lays the theoretical groundwork for laboratory probing of extreme astrophysical environments [5,6]; and for QED applications of laser-driven particle beams, such as gamma-ray radiography for materials science and fundamental nuclear physics [7].

1. S Bulanov, “Oncological Hadrontherapy with Laser Ion Accelerators,” Phys. Lett. A, 299, 240 (2002).

2. A R Bell and J G Kirk, "Possibility of Prolific Pair Production with High-Power Lasers," Phys. Rev. Lett., 101, 200403 (2008).

3. C P Ridgers et al, "Dense Electron-Positron Plasmas and Ultraintense $\gamma$ rays from LaserIrradiated Solids,” Phys. Rev. Lett., 108, 165006 (2012).

4. M C Levy et al, "QED-driven laser absorption,” in prep.

5. A N Timokhin, "Time-dependent Pair Cascades in Magnetospheres of Neutron Stars - I. Dynamics of the Polar Cap Cascade with No Particle Supply from the Neutron Star Surface,” Mon. Not. R. Astron. Soc., 408, 2092 (2010).

6. S V Bulanov, "On the Problems of Relativistic Laboratory Astrophysics and Fundamental Physics with Super Powerful Lasers,” Plasma Phys. Rep., 41, 1 (2015).

7. K W D Ledingham, “Applications for Nuclear Phenomena Generated by Ultra-Intense Lasers,” Science, 300, 1107 (2003). 


\section{Simulations of Inverse Compton Scattering as Diagnostic for Plasma Wakefield Electrons at FLASHForward}

Simon Bohlen ${ }^{1}$, Matthew Streeter ${ }^{1}$, Alexander Aschikhin ${ }^{1}$, Zhanghu Hu ${ }^{1}$, Laura di Lucchio ${ }^{1}$, Alberto Martinez de la Ossa ${ }^{1}$, Bernhard Schmidt ${ }^{1}$ and Jens Osterhoff ${ }^{1}$

${ }^{1}$ Deutsches Elektronen Synchrotron, DESY, Notkestrasse 85, 22607, Hamburg, Germany Simon.Bohlen@desy.de

FLASHForward [1] is a beam-driven plasma wakefield accelerator located at Deutsches Elektronen Synchrotron (DESY) in Hamburg, Germany. Within the FLASHForward project, laser-driven as well as beam-driven plasma waves enable acceleration of electron beams with energies from tens of $\mathrm{MeV}$ to a few $\mathrm{GeV}$. The characterization of these electrons is important to control and improve this acceleration technique.

The production of inverse Compton scattering (ICS) offers a possibility to measure electron beam parameters due to the dependence of the produced photons on the electron parameters. A numerical study of ICS radiation produced in experiments at FLASHForward was performed, using an ICS simulation code [2] and the results from particle-in-cell simulations [3,4]. The possibility of determining electron beam properties from measurements of the $\gamma$-ray source was explored for a wide range of experimental conditions.

The simulations show that the measurement of electron spectrum and divergence is in principle possible with the detection of ICS photons. In addition, transverse probing of the electron beam using ultra-short laser pulses allows to obtain longitudinal information about the electron beam in multi shot experiments. However, the detection of the produced ICS radiation, with photon energies of several $\mathrm{MeV}$ for the electron beams of interest, remains challenging.

1. A. Aschikhin et al. "The FLASHForward facility at DESY," Nucl. Instruments Methods Phys. Res. Sect. A Accel. Spectrometers, Detect. Assoc. Equip. 806 (2016).

2. W. J. Brown and F. V. Hartemann, "Three-dimensional time and frequency-domain theory of femtosecond x-ray pulse generation through Thomson scattering," Phys. Rev. Spec. Top. Accel. Beams, 7, 060703 (2004).

3. R. A. Fonseca et al., "OSIRIS: A Three-Dimensional, Fully Relativistic Particle in Cell Code for Modeling Plasma Based Accelerators," Computational Science - ICCS 2002, 2331 (2002).

4. T. Mehrling et al., "HiPACE: a quasi-static particle-in-cell code," Plasma Phys. Control. Fusion 56, 8 (2014). 


\title{
Production of Short-lived Positron Emitting Isotopes following the Rapid Acceleration of Laser Ablation Plumes with a Pulsed-Power z-pinch Plasma Driver
}

\author{
Aaron Covington ${ }^{1}$, Austin Anderson ${ }^{1}$, W. Alex Angermeier ${ }^{1}$, Nuvraj Bilkhu ${ }^{1}$, Tim Darling ${ }^{1}$, \\ Vern Davis ${ }^{1}$, Eric Dutra ${ }^{2}$, Jeremy Iracabal ${ }^{1}$, Erik McKee ${ }^{1}$ Jeff Thompson $^{1}$ and Piotr Wiewior ${ }^{1}$ \\ ${ }^{1}$ Nevada Terawatt Facility, Physics Department, University of Nevada Reno, \\ Reno, NV 89557, USA \\ acovington@unr.edu,austinanderson@unr.edu,alex.angermeier12@gmail.com, \\ nkbilkhu94@gmail.com,darling@unr.edu,vdavis@unr.edu,jeremyi@unr.edu, \\ mckee2@unr.edu,thompsonj@unr.edu,pwiewior@unr.edu. \\ ${ }^{2}$ National Securities Technologies, Livermore Operations, \\ P.O. Box 2710, Livermore, \\ CA, 94551-2710, USA \\ DutraEC@nv.doe.gov
}

Recently, it has been suggested that z-pinch plasma generators might be a powerful source for production of $\mathrm{MeV}$ ions that can be used to activate positron emission (PE) isotopes [1]. A recent experimental effort has been initiated at the Nevada Terawatt Facility to follow up on this suggestion and produce short-lived PE isotopes by accelerating ions in a laser ablation plume to $\mathrm{MeV}$ kinetic energies with a pulsed power driver. Following acceleration, the ion beams collide with an ion beam catcher wherein $(\mathrm{p}, \mathrm{n})$ and $(\mathrm{d}, \mathrm{n})$ reactions can occur. Experiments were performed by focusing a $\sim 20 \mathrm{~J}, 0.8 \mathrm{~ns}$ pulse from our TW-class $1057 \mathrm{~nm}$ "Leopard" laser onto solid polyethylene $\left(\mathrm{C}_{2} \mathrm{H}_{4}\right)_{n}$ or $\left(\mathrm{C}_{2} \mathrm{D}_{4}\right)_{n}$ targets centered on the cathode of our $2 \mathrm{TW}$ "Zebra" $\mathrm{z}$ pinch plasma accelerator. Once the plume had expanded sufficiently to fill the cathode-anode gap, the 100ns rise-time z-pinch was triggered, and the plume was pinched on the symmetric zaxis and then accelerated back onto the $\sim 2.5 \mathrm{~cm}$ diameter cathode. Cathode activation materials included polyethylene or Boron Nitride (BN) disks.

Following the activation, the disks were removed from the vacuum system and placed between pairs of gamma detectors consisting of scintillation plastic and NaI photomultiplier tubes. Signals from the tubes were then amplified, discriminated and measured with a coincidence system. Coincidence events were then logged with a data acquisition computer. The counting system was triggered by Z-pinch pulse in order to establish the $t=0$ creation time. $\mathrm{A}^{22} \mathrm{Na}$ calibration source of known activity was used to calibrate the coincidence count rates in the system.

A number of different $\mathrm{PE}$ isotopes have been activated through different channels including ${ }^{10} \mathrm{~B}(\mathrm{~d}, \mathrm{n}){ }^{11} \mathrm{C},{ }^{11} \mathrm{~B}(\mathrm{p}, \mathrm{n}){ }^{11} \mathrm{C},{ }^{12} \mathrm{C}(\mathrm{d}, \mathrm{n}){ }^{13} \mathrm{~N}$ and ${ }^{14} \mathrm{~N}(\mathrm{~d}, \mathrm{n}){ }^{15} \mathrm{O}$. These have been identified by comparison to known PE decay half-lives. Preliminary measurements indicate that an impulse activation of these types short-lived isotopes is possible. Moreover, this technique holds promise for the activation of large quantities of short-lived isotopes $(\leq 1 \mathrm{~min})$ for next generation positron emission tomography (PET) scans and other applications. Results from preliminary experiments will be presented and follow on experimental plans discussed.

1. D. Klir, et al, "Deuterium z-pinch as a powerful source of multi-MeV ions and neutrons for advanced applications," Physics of Plasmas, 23, 032702 (2016). 


\title{
Designer spectral phases for stretching and compression of intense laser pulses
}

\author{
Marcos Dantus $^{1.2}$, and Vadim V. Lozovoy ${ }^{1}$ \\ ${ }^{1}$ Department of Physics and Astronomy, Michigan State University, East Lansing, Michigan \\ 48824,USA,dantus@msu.edu,lozovoy@msu.edu \\ ${ }^{2}$ Department of Chemistry, Michigan State University, East Lansing, Michigan 48824, USA, \\ dantus@msu.edu
}

Energy scale up of ultrafast laser sources relies on temporal stretching of the pulses prior to amplification followed by pulse compression. Our research group has focused on improving the quality of pulse compression by developing pulse shapers that automatically measure and compress the pulses to their transform-limited value. ${ }^{1}$ As petawatt and exawatt lasers with pulse energies in the hundreds and even thousands of joules are considered to address the generation of zeptosecond pulses, the compressors needed for such chirped-pulse amplification sources become extremely expensive. The expense stems from the need for high-quality gratings and mirrors with areas exceeding $1 \mathrm{~m}^{2}$. To operate properly, the compressors require precise alignment of large optics inside a vacuum chamber. In view of the technical challenges, we question if a fresh perspective on pulse compression may lead to new methodology.

In our minds, pulse compression is the process by which all frequencies within the spectrum of the pulse are brought in phase. As the simplest approximation, we consider a frequency domain approach in which out-of-phase components are brought into phase by introducing a $\pi$ step. This implies that, instead of advancing or delaying frequencies by picoseconds, they are only delayed by the time corresponding to half a wavelength, or roughly a couple of femtoseconds. The resulting binary spectral phases, containing values of zero and $\pi$ across the spectrum, are evaluated numerically as well as experimentally. This work is an extension of previous work from our group on mitigating self-action processes using binary-phase shaping. ${ }^{2}$

We have explored several different strategies for binary-phase compression through numerical modeling. We will present results for binary-phase pulse compression of factors of a million back to transform limit; and experimentally obtain results for pulse compression of a factor of one hundred in close agreement with numerical calculations. The new concept of binary-phase stretching and compression, if implemented in a multi-layer optic, would eliminate the need for traditional pulse stretchers and compressors.

1. Y. Coello, V. V. Lozovoy, T. C. Gunaratne, B. Xu, I. Borukhovich, C. -h. Tseng, T. Weinacht, and M. Dantus, "Interference without an interferometer: a different approach to measuring, compressing, and shaping ultrashort laser pulses," J. Opt. Soc. Am. B 25, A140 (2008).

2. G. Rasskazov, A. Ryabtsev, V.V. Lozovoy, and M. Dantus, "Mitigating self-action processes with chirp or binary phase shaping," Optics Letters 41, 131 (2016). 


\title{
A New Research Project on Laser-Driven Neutron sources and applications at ILE, Osaka University
}

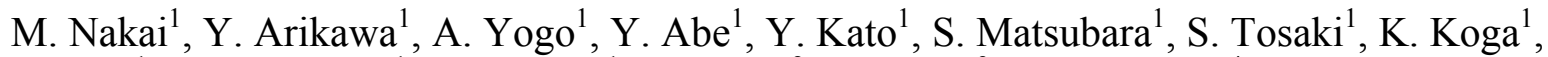 \\ N. Iwata ${ }^{1}$, H. Nagatomo ${ }^{1}$, A. Morace ${ }^{1}$, K. Mima ${ }^{2}$, Y. Otake ${ }^{3}$, S. Miyamoto ${ }^{4}$ and H. Nihsimura ${ }^{1}$ \\ ${ }^{1}$ Institute of Laser Engineering, Osaka University, Suita 565-0871, Osaka, Japan \\ mitsuo@ile.osaka-u.ac.jp, arikawa-y@ile.osaka-u.ac.jp,yogo-a@ile.osaka-u.ac.jp, \\ abe-y@ile.osaka-u.ac.jp,kato-y@ile.osaka-u.ac.jp,matsubara-s@ile.osaka-u.ac.jp, \\ tosaki-s@ile.osaka-u.ac.jp,koga-k@ile.osaka-u.ac.jp,iwata-n@ile.osaka- \\ u.ac.jp,naga@ile.osaka-u.ac.jp,morace@ile.osaka-u.ac.jp,nishimu@ile.osaka-u.ac.jp \\ ${ }^{2}$ The Graduate School for the Creation of New Photon Industries, Hamamatsu, Shizuoka 431 - \\ 1202,Japan,mima@ile.osaka-u.ac.jp \\ ${ }^{3}$ RIKEN,Wako 351-0198, Saitama, Japan, yotake@riken.jp \\ ${ }^{4}$ Laboratory of Advanced Science and Technology for Industry, University of Hyogo, \\ Ako-gun 678-1205,Hyogo,JAPAN,miyamoto@lasti.u-hyogo.ac.jp
}

Neutron sources are getting important in the various fields of applications as well as nuclear energy developments. Success of the neutron application for the natural science, biology, medicine and engineering at the large facilities, such as J-PARC, stimulated the industrial needs for compact neutron sources. Laser driven neutron sources have unique properties of a tiny, bright pulse source which is suitable for a point projection imaging system. Following the preliminary study [1] and basic experiments, we have initiated a project to get a clear perspective of the laser driven neutron sources for the dynamic neutron radiography. Final goal of the project is to demonstrate the possibility of the compact neutron imaging system using a repetitive high intensity laser.

The tasks of the project are 1) investigation of the scaling and optimization of the neutron generation mechanisms, 2) development of large formatted neutron imager with the 1-mm spatial resolution, 3) development of the continuous and precise targets supplying system, 4) design of the neutron moderators for the system which provide a proper neutron energy distribution.

The neutron generation processes pursued are nuclear fusion of accelerated hydrogen isotopes, proton capture reaction with light elements such as $\mathrm{Li}$ and $\mathrm{Be}$, photonuclear reactions of heavy elements. As a first phase of the project, we experimentally investigated the energy scaling of the photo-nuclear reaction and proton acceleration for the ion-driven neutron reactions by using the LFEX laser at ILE, Osaka University. Here we have demonstrated the more effective acceleration than the usual ponderomotive scaling owing to the anomalous electron heating by using the laser pulse as long as $1 \mathrm{ps}$ for the first time. As for the development of an imaging device, a large scintillation detector with the sensitive area of $40 \mathrm{~cm}$ x $60 \mathrm{~cm}$ was constructed. An aluminum honeycomb plate was filled with liquid scintillator and coupled to a gated image intensifier and a CCD camera. Temporally discriminated x-ray and neutron imaging of $20 \mathrm{~cm}$ thick concrete block were successfully demonstrated by using an electron linear accelerator facility in Osaka University.

This project is supported by the Japan Science and Technology Agency (JST) and partly by the Japan Society for the Promotion of Science under the contracts of Grant-in-Aid for Scientific Research (A) No. 2624043.

1. S. Nakai et al., "Industrial applications of laser neutron source", Journal of Physics, Conference series 244, 042027(2010). 


\section{High-vacuum Laser-Driven Electron Accelerator for Compact Radiation Sources}

Seong Hee Park ${ }^{1}$, Jaehoon Kim ${ }^{2}$, Woo-Je Ryu ${ }^{1,3}$, Kyung Nam Kim ${ }^{1}$, Younghun Hwangbo ${ }^{2}$, Kitae Lee $^{1}$, Young Uk Jeong ${ }^{1}$, Nikolay A. Vinokuro ${ }^{4}$

${ }^{1}$ Center of Quantum-Beam-based Radiation Research, Korea Atomic Energy Research Institute, Daejeon, 305-358, Rep. Korea shpark@kaeri.re.kr,rwj4292@kaeri.re.kr,kkn2003@kaeri.re.kr,klee@kaeri.re.kr, yujung@kaeri.re.kr

${ }^{2}$ Applied Electromagnetic Wave Research Center, Korea Electrotechnology Research Institute, Ansan,Geyonggi,426-170,Rep.Korea,jkim@keri.re.kr,hwangboda@keri.re.kr

${ }^{3}$ Department of Physics, Hannam University, Daejeon, 111-111, Rep. Korea ${ }^{4}$ Budker Institute of Nuclear Physics,SB RAS, Academician Lavrentyev St.11, Novosibrisk, Russia,vinokurov@inp.nsk.su

A compact radiation source using laser-driven electron accelerator is being under development at KAERI. Due to short acceleration length, a laser-driven electron accelerator can minimize its size and cost with localized shielding for many applications. In addition to issues in shot-to-shot stability and beam quality, the vacuum condition using gas targets is poor for all-inone radiation source or an injector. With the $30 \mathrm{TW}$ fs laser in KAERI, we demonstrated laser electron acceleration with its energy of $55 \mathrm{MeV}$ using metal target. Unlike typical gas target, high vacuum below $10^{-5}$ torr can be maintained using laser-induced pre-plasma. The success of high vacuum laser electron accelerator using metallic plasma opens the possibility of all-in-one compact radiation source in small laboratories where they need easy accessibility. We present the experimental results of laser-accelerated electron beam using metal target and the prospect of compact radiation sources, such as, gamma-rays, synchrotron radiation, and $\mathrm{THz}$ radiation, for nuclear and material applications. 


\title{
Ignatovsky Diffraction - Calculating Vector Fields in an Arbitrarily Tight Laser Focus since 1920
}

\author{
Justin Peatross and Michael Ware \\ Brigham Young University, Dept. of Physics and Astronomy, Provo, UT, USA \\ peat@byu.edu,ware@byu.edu
}

Ultra-intense laser systems frequently employ parabolic mirrors with tight focusing geometries. The detailed trajectories of charged particles being propelled through different regions of the focus depend on the precise local vector forces distributed throughout the focus. An accurate accounting of the vector electric and magnetic field distribution in the focal region is needed to precisely model the trajectories.

We highlight the under-appreciated (and under-used) work of V. S. Ignatovsky [1] which models vector diffraction for a beam focused by a parabolic mirror or by a lens. Ignatovsky published his work in 1920. Unfortunately, Ignatovsky was executed together with his wife by the Soviets, but his work has influenced the development of vector diffraction in the microscopy community for nearly a century. We provide a streamlined and accessible derivation of Ignatovksy's results and demonstrate its practicality for use in high-intensity laser physics. For an azimuthally symmetric beam with uniform polarization, the diffraction integral collapses to one dimension, which can be performed numerically with reasonable efficiency.

Ignatovsky's formula, which satisfies Maxwell's equations exactly, applicable to an incident monochromatic beam (polarized along $x$ ) focused by an ideal parabola, may be written as

$$
\begin{aligned}
& \vec{E}(x, y, z)=-i k f e^{i(k f-\omega t)}\left[\hat{x}\left(I_{0}+\frac{x^{2}-y^{2}}{x^{2}+y^{2}} I_{2}\right)+\hat{y} \frac{2 x y}{x^{2}+y^{2}} I_{2}-i \hat{z} \frac{x}{\sqrt{x^{2}+y^{2}}} I_{1}\right] \\
& \vec{B}(x, y, z)=-i \frac{k f}{c} e^{i(k f-\omega t)}\left[\hat{x} \frac{2 x y}{x^{2}+y^{2}} I_{2}+\hat{y}\left(I_{0}-\frac{x^{2}-y^{2}}{x^{2}+y^{2}} I_{2}\right)-i \hat{z} \frac{y}{\sqrt{x^{2}+y^{2}}} I_{1}\right. \\
& I_{0} \equiv \int_{0}^{\pi} d \theta E_{i}\left(\rho^{\prime}\right) \sin \theta J_{0}\left(k \sqrt{x^{2}+y^{2}} \sin \theta\right) e^{i k z \cos \theta} \\
& I_{1} \equiv 2 \int_{0}^{\pi} d \theta E_{i}\left(\rho^{\prime}\right) \sin \theta \sqrt{\xi(\theta)} J_{1}\left(k \sqrt{x^{2}+y^{2}} \sin \theta\right) e^{i k z \cos \theta} \\
& I_{2} \equiv \int_{0}^{\pi} d \theta E_{i}\left(\rho^{\prime}\right) \sin \theta \xi(\theta) J_{2}\left(k \sqrt{x^{2}+y^{2}} \sin \theta\right) e^{i k z \cos \theta}
\end{aligned}
$$$$
\vec{B}(x, y, z)=-i \frac{k f}{c} e^{i(k f-\omega t)}\left[\hat{x} \frac{2 x y}{x^{2}+y^{2}} I_{2}+\hat{y}\left(I_{0}-\frac{x^{2}-y^{2}}{x^{2}+y^{2}} I_{2}\right)-i \hat{z} \frac{y}{\sqrt{x^{2}+y^{2}}} I_{1}\right]
$$

with $\xi(\theta) \equiv(1-\cos \theta) /(1+\cos \theta)$. The radius $\rho^{\prime}=2 f \sqrt{\xi(\theta)}$ shown in the figure parameterizes the incident field distribution at the parabolic mirror. The field amplitude of a collimated Gaussian beam, for example, can be described by $E_{i}\left(\rho^{\prime}\right)=E_{0}^{\prime} e^{-\rho^{\prime 2} / w^{2}}$, where $w$ is the beam width at the mirror. The focal length of the mirror is $f$. The point $(x, y, z)=(0,0,0)$

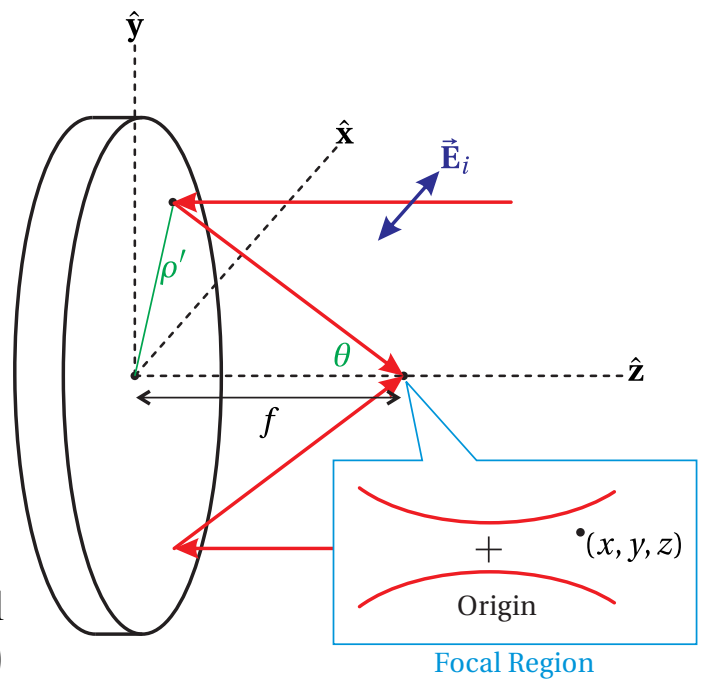


corresponds to the center of the focus. The above formula accurately models the fields anywhere in the beam, before or after the focus.

Although some in the microscopy community have used Ignatovsky diffraction, many in the laser community have sought alternative vector models of a laser focus, apparently without the benefit of Ignatovsky's work. A variety of models have been offered, which often differ markedly from each other. A broad criticism that we make against many of these models is that they start from an assumed field distribution in the focal region and attempt to develop vector fields (consistent with Maxwell's equations) in the surrounding region. This approach is at odds with the fact that no experimenter directly controls the focal field distribution. Rather, experimenters typically diagnose and manipulate their incident beam to control the fields at the focusing optic before it converges, diffracts, and interferes to form the focal fields. Moreover, the ability to directly measure vector components of the fields in an intense focus is extremely limited. This makes Ignatovsky diffraction, where the incident field is defined at a focusing optic rather than inside the focus, much more natural and relevant to experimental work.

Still, for applications such as computing relativistic trajectories of charged particles in a tightly focused intense beam, one would ideally like a closed analytic formula that adequately represents the vector field components to avoid repeatedly evaluating the $I_{0}, I_{1}$, and $I_{2}$ integrals at various position within the interaction region. We find that a paraxial vector model proposed by Erikson and Singh [2] best agrees with Ignatovsky diffraction (down to f/2 optics):

$$
\vec{E}=E_{0}\left[\hat{x}+\frac{x y}{2\left(z_{0}+i z\right)^{2}} \hat{y}-i \frac{x}{z_{0}+i z} \hat{z}\right] \psi_{0} e^{i(k z-\omega t)}, \vec{B}=\frac{E_{0}}{c}\left[\frac{x y}{2\left(z_{0}+i z\right)^{2}} \hat{x}+\hat{y}-i \frac{y}{z_{0}+i z} \hat{z}\right] \psi_{0} e^{i(k z-\omega t)}
$$

where $\psi_{0}=\frac{z_{0}}{z_{0}+i z} \exp \left(-\frac{k}{2} \frac{x^{2}+y^{2}}{z_{0}+i z}\right)$ is the usual scalar lowest-order Gaussian mode with Rayleigh range $z_{0}$. On the other hand, a frequently employed iterative scheme first introduced by Lax in 1975 ,[3] with the intent of improving beyond the paraxial limit, actually worsens agreement with Ignatovsky. We note also that the Lax expansion produces undesirable divergences in the far field, making that program suspect. This work was supported in part by the Air Force Office for Scientific Research (FA9550-11-1-0157) with no expressed or implied endorsement.
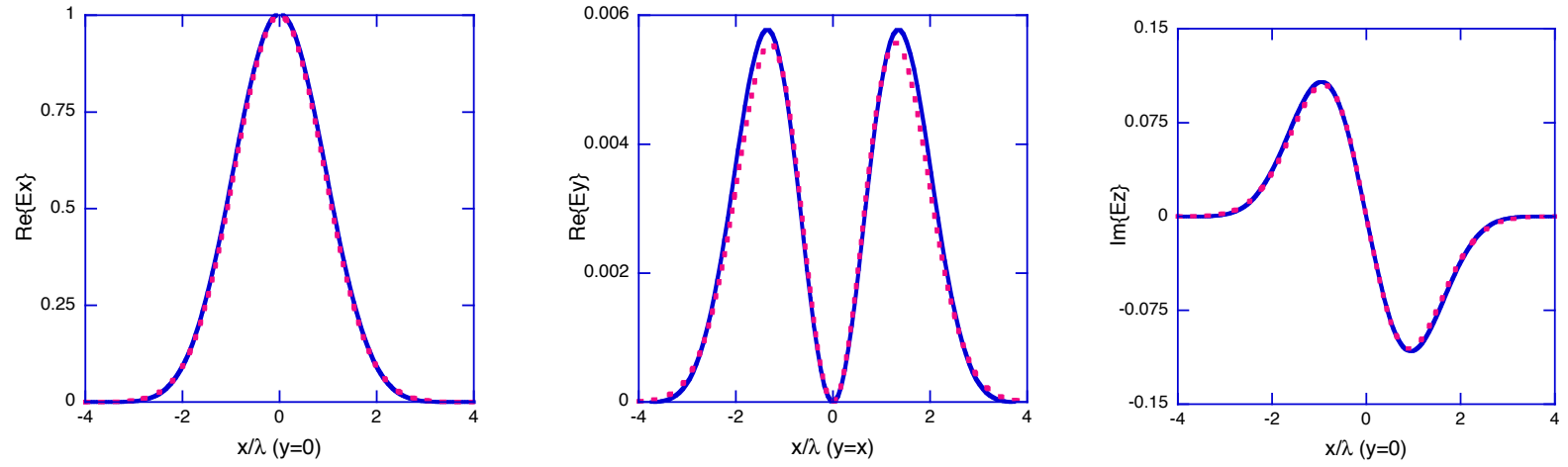

Fig. $\mathrm{x}, \mathrm{y}, \mathrm{z}$ components of electric field for on-axis parabolic $\mathrm{f} / 2$ focusing at $z=0$ : Ignatovsky (solid), Singh (dashed).

1. V. S. Ignatovsky, "Diffraction by a Parabolic Mirror Having Arbitrary Opening," Trans. Opt. Inst. Petrograd 1, paper 5 (1920).

2. W. L. Erikson and S. Singh, "Polarization Properties of Maxwell-Gaussian Laser Beams," Phys. Rev. E 49, 5778-5786 (1994).

3. M. Lax, W. H. Louisell, and W. B. McKnight, "From Maxwell to paraxial wave optics," Phys. Rev. A 11, 1365-1370 (1975). 


\section{Diagnosis of Compton $\gamma$-ray sources based on a laser wakefield accelerator and high- energy electron radiography}

R. Qi, J. S. Liu, W.T. Wang, C.H. Yu, W.T. Li, C. Wang, Z.J. Zhang, J.Q. Liu, Z.Y. Qin, M. Fang, Y. Xu, Y.X. Leng, R.X.Li, Z.Z.Xu

State Key Laboratory of High Field Laser Physics, Shanghai Institute of Optics and Fine Mechanics (SIOM), Chinese Academy of Sciences, Shanghai 201800, China qrong0353@163.com, michaeljs_liu@siom.ac.cn

A cascaded laser wakefield accelerator was designed to generate high-quality monoenergetic e-beams, which were bound to head-on collide with the intense driving laser pulse via the reflection of a $20-\mu \mathrm{m}$-thick Ti foil (Inverse Compton scattering). We diagnosed the generation of tunable quasi-monochromatic $\mathrm{MeV} \gamma$-rays by using a $\mathrm{Lu}_{2} \mathrm{SiO}_{4}(\mathrm{LSO})$-crystal scintillator detector. A peak brilliance of $\sim 3 \times 10^{22}$ photons $\mathrm{s}^{-1} \mathrm{~mm}^{-2} \operatorname{mrad}^{-2} 0.1 \% \mathrm{BW}$ at $1 \mathrm{MeV}$ was achieved, which was one order of magnitude higher than ever reported value of its kind in $\mathrm{MeV}$ regime to the best of our knowledge [1].

Also, we studied the high-energy electron beam radiography of two types of microstructures in a dense material both on experiment and numerical simulations by using a high-energy electron beam. According to the laser-wakefield electron acceleration mechanism, the accelerated electron beam inherits high-energy $(\mathrm{MeV} \sim \mathrm{GeV})$ and high time-resolution characteristics from femtosecond high-intensity laser pulse. A high-energy electron pulse with several femtosecond duration is the optimal probe tool to analyze the ultrafast process, in dense material i.e. the fast ignition target core.

1. C. $\mathrm{H} . \mathrm{Yu}$, et al.,"Ultrahigh brilliance quasimonochromatic $\mathrm{MeV} \gamma$-rays based on selfynchronized all-optical Compton scattering", Scientific Reports, 6, 29518 (2016). 


\title{
Brilliant Gamma-Ray Emission from Near-Critical Plasma Interaction with Ultraintense Laser Pulses
}

\author{
B. Qiao ${ }^{1, *}$, X. T. He ${ }^{1}$, M. Zepf ${ }^{2}$, H. X. Chang ${ }^{1}$, X. Q. Yan ${ }^{1}$, T. W. Huang ${ }^{1}$ \\ ${ }^{1}$ Center for Applied Physics and Technology, Peking University, Beijing, P. R. China \\ *Email:bqiao@pku.edu.cn \\ ${ }^{2}$ Department of Physics and Astronomy, Queen's University Belfast, Belfast, United Kingdom
}

$\gamma$-ray is the electromagnetic radiation with extremely high frequency and high photon energy, which has a broad range of applications in industry, material science, nuclear physics, astrophysics and so on. In this talk, I shall report on a novel resonant acceleration scheme [1, 2, 3] for generating ultradense relativistic electron bunches in helical motions and hence emitting brilliant vortical $\gamma$-ray pulses in the quantum electrodynamic (QED) regime by using a nearcritical plasma interaction with ultraintense circularly polarized (CP) laser pulses. In this QED regime, the combined effects of the radiation reaction recoil force and the self-generated axial and azimuthal magnetic fields results in not only trapping of a great amount of electrons in laserproduced plasma channel, but also significant broadening of the resonant bandwidth between laser frequency and that of electron betatron oscillation in the channel, which eventually leads to formation of an ultradense electron bunch under resonant helical acceleration in CP laser fields. Both the particle number and energy of such electron bunches are much larger than those under only direct laser acceleration (DLA) by linearly polarized lasers. Three-dimensional PIC simulations show that brilliant $\gamma$-ray pulses with unprecedented power of $6.7 \mathrm{PW}$ and brightness of $10^{25}$ photons $/ \mathrm{s} / \mathrm{mm}^{2} / \mathrm{mrad}^{2} / 0.1 \% \mathrm{BW}$ (at $15 \mathrm{MeV}$ ) are produced at laser intensity $1.9 \times$ $10^{23} \mathrm{~W} / \mathrm{cm}^{2}$. To the best of our knowledge, this is the $\gamma$-ray source with the highest peak brightness in tens- $\mathrm{MeV}$ regime ever reported in the literature.

1. H. X. Chang, B. Qiao et al., "Generation of overdense and high-energy electron-positron-pair plasmas by irradiation of a thin foil with two ultraintense lasers", Phys. Rev. E 92, 053107 (2015).

2. T. W. Huang, A. P. L. Robinson, C. T. Zhou, B. Qiao et al., "Characteristics of betatron radiation from direct-laser-accelerated electrons", Phys. Rev. E 93, 063203 (2016).

3. H. X. Chang, B. Qiao et al., "Brilliant Petawatt Gamma-Ray Pulse Generation in Quantum Electrodynamic Laser-Plasma Interaction”, Physical Review Letters, under Review (2016). 


\title{
Positron Acceleration In Plasma Bubble Wakefield Driven By An Ultraintense Laser
}

\author{
Hai-Bo Sang, BaiSong Xie, YaJuan Hou and Wei Cheng \\ College of Nuclear Science and Technology, Beijing Normal University, \\ Beijing 100875, China \\ sanghb@bnu.edu.cn,bsxie@bnu.edu.cn,1317277521@qq.com,chengwei@bnu.edu.cn
}

\begin{abstract}
:
The dynamics of positrons accelerating in electron-positron-ion plasma bubble fields driven by an ultraintense laser is investigated. The bubble wakefield is obtained theoretically when laser pulses are propagating in the electron-positron-ion plasma. To restrict the positrons transversely, an electron beam is injected. Acceleration regions and non-acceleration ones of positrons are obtained by the numerical simulation. It is found that the ponderomotive force causes the fluctuation of the positrons momenta, which results in the trapping of them at a lower ion density. The energy gaining of the accelerated positrons is demonstrated, which is helpful for practical applications.
\end{abstract}

I. Modification of Plasma Bubble Field

The elliptic bubble wall in cylindrical coordinates $(x, r, \theta)$ is given by $\frac{\xi^{2}}{\nu^{2}}+r^{2}=R^{2}[1-3]$, where the quasi-static approximation $x-v_{0} t$ is used, $v_{0}$ is the bubble velocity, $R$ is the transverse radius of the elliptic bubble, $n$ denotes the ratio of longitudinal radius to the transverse one. The model of the bubble field is based on the Maxwell's equations and the hydrodynamic equations. An electron beam is injected into the background plasma towards the laser pulse to restrict the positrons from diverging transversely. It is assumed that the radius of the electron beam is $a$, the transversal surface of the wire is $s$, the electron density of the beam is $n_{e}{ }^{*}$, and the velocity of it is $v_{e}{ }^{*}$. Then the electric and magnetic fields $\boldsymbol{E}, \boldsymbol{B}$ in the bubble is:

$$
\begin{aligned}
& E_{x}=a x / 2, E_{r}=B_{x}=B_{r}=0, \\
& B_{\theta}= \begin{cases}-\frac{\alpha}{4} r+C^{*} r & \text { if } r<a \\
-\frac{\alpha}{4} r+D^{*} \frac{1}{r} & \text { if } r \geq a,\end{cases} \\
& E_{r}=\frac{\alpha}{2} r-D^{*} \frac{1}{r} .
\end{aligned}
$$

where $\alpha$ is the density ratio of ions to electrons, and $C^{*}=\frac{\mu_{0} n_{e *} e \bar{v}_{e *} s}{2 \pi a^{2}}, D^{*}=\frac{\mu n_{e *} e v_{e *} s}{2 \pi}$.

\section{Numerical Results}

With the help of numerical solutions of EOM, the optimum initial phase regions of $\left(\xi_{0}, p_{x 0}\right)$ for positron acceleration are obtained and the acceleration regions and non-acceleration ones of positrons are named as region I and region II, respectively, which are different with the effect of ponderomotive field. The features of the accelerated positrons in these phase regions are also different due to the ponderomotive force. It is found that the phase trajectories of positrons can be enclosed with $F p \neq 0$ at lower ion density, which means that the positrons are trapped. Besides, it is concluded that positrons with initial position and momentum in region I near the lower boundaries can be more easily accelerated and gain more energy. The results of positron acceleration in this work are of great significance for practical applications. 

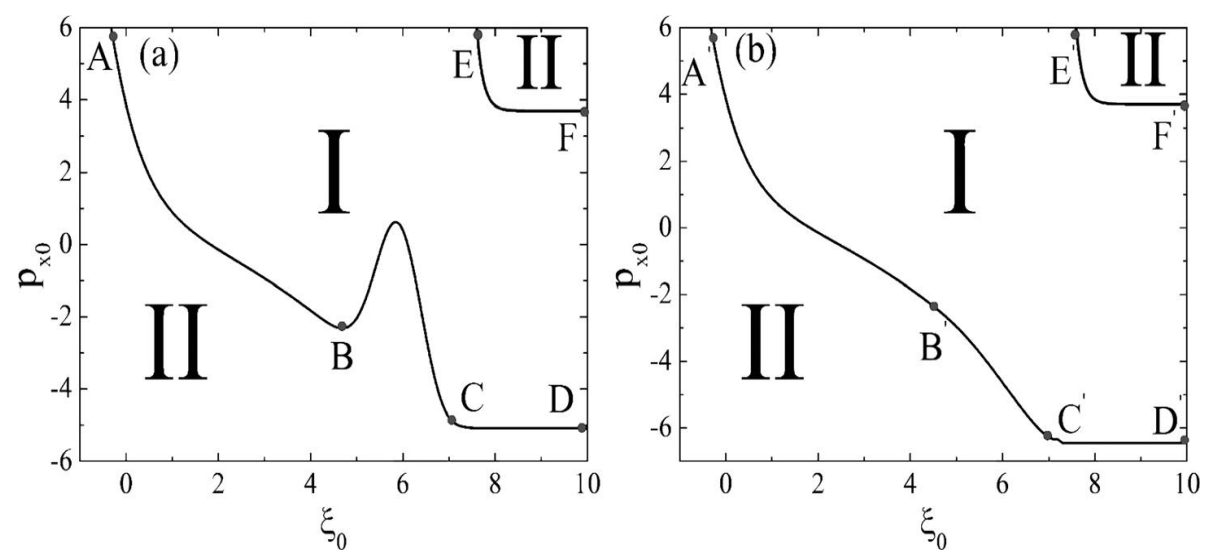

Fig. 1. The parameter phase regions of positron acceleration in $\xi_{0} p_{x 0}$ plane at background ion density $n_{i 0}=0.9$. (a) The case of $F p \neq 0$. (b) The case of $F p=0$. Region I in both figures are acceleration regions, and region II in both figures are non-acceleration ones.
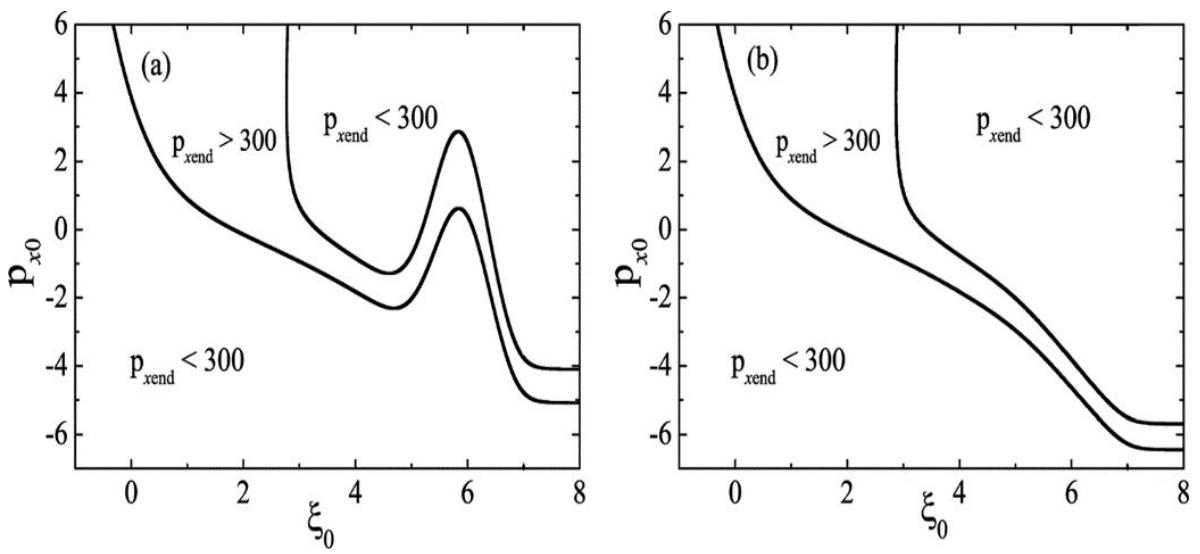

Fig. 2. The parameter phase portraits of positrons in $\xi_{0} p_{x 0}$ plane at $n_{i 0}=0.9$ is plotted by whether the final momentum of positrons $p_{x e n d}>300$ or $p_{x e n d}<300$. (a) The case of $F p$ $\neq 0$. (b) The case of $F p=0$.

1. H. C. Wu, B. S. Xie, S. Zhang, X. R. Hong, X. Y. Zhao, and M. P. Liu, Bubble core field modification by residual electrons inside the bubble, Phys. Plasmas, 17, 113103 (2010).

2. I. Kostyukov, A. Pukhov, and S. Kiselev, Phenomenological theory of laser-plasma interaction in "bubble" regime, Phys. Plasmas, 11, 5256 (2004).

3. D. Lu, X. Y. Zhao, B. S. Xie, M. Ali Bake, H. B. Sang, and H. C. Wu, Electrons trajectories around a bubble regime in intense laser plasma interaction, Phys. Plasmas, 20, 063104 (2013) 
Collimated gamma-ray beam produced by laser-matter interaction in the grazing incidence regime

\author{
D.A. Serebryakov ${ }^{1,2}$, E.N. Nerush ${ }^{1,2}$, I.Yu. Kostyukov ${ }^{1,2}$ \\ ${ }^{1}$ Institute of Applied Physics of the Russian Academy of Sciences, Nizhny Novgorod, Russia \\ dms@appl.sci-nnov.ru,nerush@appl.sci-nnov.ru,kost@appl.sci-nnov.ru \\ ${ }^{2}$ Lobachevsky State University of Nizhny Novgorod
}

Relativistic laser-solid interaction is currently expected to be a promising alternative to traditional gamma-ray sources (e.g. based on radioactive decay, bremsstrahlung of relativistic electrons, or Compton backscattering). Depending on laser and plasma parameters, light interacts with targets differently and various interaction regimes occur [1, 2]. Usually, the best laser-togamma-ray energy conversion efficiency corresponds to linearly polarized laser pulse interacting with a target which electron density $n_{e}$, normalized to the critical density $m \omega^{2} /\left(4 \pi e^{2}\right)$, is of the order of dimensionless laser amplitude $a_{0}=e E_{0} /(m c \omega)$. It is also known that oblique incidence of $p$-polarized laser pulses can substantially increase the hard photon generation efficiency [3, 4].

However, best gross efficiency does not usually result in narrow angular distribution of radiation and, hence, high brilliance. We show that use of a grazing incident laser pulses (with incidence angles in the range $70-85^{\circ}$ ) dramatically improves brightness of a gamma-ray source in comparison to other regimes. The best parameters in the series of particle-in-cell simulations $(\theta=$ $81^{\circ}, n_{e}=50 n_{c r}$ for $\left.a_{0}=55\right)$ result in the peak brilliance $=5.4 \times 10^{23}$ photons $/$ $\left(\mathrm{s} \cdot \mathrm{mrad}^{2} \cdot \mathrm{mm}^{2} \cdot 0.1 \% \mathrm{BW}\right)$ at $1 \mathrm{MeV}, 1.36 \times 10^{23}$ photons $/\left(\mathrm{s} \cdot \mathrm{mrad}^{2} \cdot \mathrm{mm}^{2} \cdot 0.1 \% \mathrm{BW}\right)$ at $10 \mathrm{MeV}$. Total number of multi-MeV photons in this case is $1.05 \times 10^{11}$ (the laser pulse energy is $12 \mathrm{~J}$ ).

We analyze electron dynamics that leads to good collimation of gamma-ray beams. It is demonstrated that the electrons are significantly accelerated in a surface wave structure, and maximum Lorentz factor of them is much greater than in the case of normal incidence or oblique incidence under moderate angles. Transition to the grazing incidence regime occurs very sharply on the interval of incidence angles from 60 to $66^{\circ}$; this is also accompanied by abrupt change in the electron phase space. Maximum electron energy and the incidence angle threshold are estimated theoretically, and they are in agreement with numerical simulation results.

1. C.P. Ridgers et al., "Dense electron-positron plasmas and ultraintense $\gamma$-rays from laserirradiated solids", Phys. Rev. Lett., 108, 165006 (2012).

2. D.A. Serebryakov et al., "Incoherent synchrotron emission of laser-driven plasma edge", Phys. Plasmas, 22, 123119 (2015).

3. D.A. Serebryakov, E.N. Nerush, "Efficient gamma-ray generation by ultra-intense laser pulses obliquely incident on a planar plasma layer", Quantum Electronics, 46 (4), 299 (2016).

4. K.Q. Pan et al., "Tunable hard x-ray source from obliquely incident intense laser interacting with overdense solid targets", Phys. Plasmas, 22, 083301 (2015). 


\title{
Waveguide, Nonlinear Optics: From laser beam to electron measurement
}

\author{
Shen Lei \\ Shanghai Institute of Applied Physics, Chinese Academy of Sciences, 2019 Jialuo Road, Jiading \\ District, Shanghai, 201800, P.R. China \\ Lshen730330@msn.com
}

The sustainable development of ultra-short laser technology has facilitated enormous progress in frontier studies of the intense laser accelerated energetic electrons and protons[1,2], the characteristics of Free Electron Lasers[3], and so on. Among these hot topics, it is an important thing how laser light is transmitted inside the different wave-guide structure. Aiming at a typical geometry structure of hollow core or the cone with medium, based on the explicit analysis of propagating modes, the transmission characteristics as the electromagnetic fields configurations, the spatial distribution of electromagnetic field components, the wave impedance and the propagation constant are analyzed. Another structure as corrugated structure is also considered by the same method. The application of different structure will also be discussed. Nonlinear properties will be considered as well. All the result will be useful for not only the laser beam itself, but the electron measurement.

1. J. Faure, Y. Glinec, A. Pukhov, et al., "A laser-plasma accelerator producing monoenergetic electron beams", Nature, 431, 541 (2004)

2. J. Fuchs, P. Antici, E.d'Humières, et al., "Laser-driven proton scaling laws and new paths towards energy increase", Nature Physics, 2(1), 48 (2006)

3. H. N. Chapman, P. Fromme, A. Barty, et al., "Femtosecond X-ray protein nanocrystallography", Nature, 470, 73 (2011) 


\title{
Gamma \& neutron production with femtosecond table top TW laser
}

\author{
I.N.Tsymbalov ${ }^{1,2}$, S.A.Shulyapov ${ }^{2}$, K.A.Ivanov ${ }^{2}$, R.V.Volkov ${ }^{2}$, A.B.Savel'ev ${ }^{2}$ \\ ${ }^{1}$ Institute for Nuclear Research RAS, Moscow, Russia \\ ${ }^{2}$ Lomonosov State University, Moscow, Russia \\ ivankrupenin2@gmail.com
}

Plasma created by femtosecond laser pulse of high intensity can be used as the brilliant source of high energy electrons, ions, X- or $\gamma$-rays and secondary neutrons. There are many mechanisms of electron acceleration on which based such a source $-\mathrm{j} \times \mathrm{B}$ and ponderomotive acceleration, resonant and stochastic heating, parametric instabilities, and even wakefield acceleration could play a dominant role. Parameters of experimental setup such as laser pulse intensity, its duration and temporal structure (pre-pulses, leading edge sharpness, ASE level and duration), type and geometry of a target determines pre-plasma scale and density, which in its turn give rise to the different acceleration mechanisms. Thus we can change plasma source characteristics controlling parameters of experimental setup. In this work we present the results of the experimental studies of the $\mathrm{MeV} \gamma$-rays generation in femtosecond laser plasma with different pre-pulse parameters on the table top TW laser system in Lomonosov State University. Additionally, we present the results of the experimental studies of the neutrons generation at photo-nuclear reactions in the secondary deuterium target. 


\title{
Control of seeding phase for the cascaded laser wakefield accelerator with
}

\section{gradient injection}

\author{
Wentao Wang, Wentao Li, Jiansheng Liu ${ }^{\text {a) }}$, Cheng Wang, Qiang Chen, Zhijun Zhang, \\ Rong Qi, Yuxin Leng, Xiaoyan Liang, Yanqi Liu, Xiaoming Lu, Cheng Wang, Ruxin Li ${ }^{\text {b) }}$ \\ and Zhizhan $\mathrm{Xu}^{\mathrm{c})}$
}

State Key Laboratory for High Field Laser Physics, Shanghai Institute of Optics and Fine Mechanics, Chinese Academy of Sciences (CAS), Shanghai 201800, China.

We demonstrated experimentally the seeding-phase control for the two-stage laser wakefield accelerator with gradient injection. By optimizing the seeding phase of electrons into the second stage, electron beams beyond $0.5 \mathrm{GeV}$ with $3 \%$ r.m.s energy spread were produced over a short acceleration distance of $\sim 2 \mathrm{~mm}$. The peak energy of the electron beam was further extended beyond $1 \mathrm{GeV}$ by lengthening the second acceleration stage to $5 \mathrm{~mm}$. Time-resolved magnetic field measurements via a magnetic-optic Faraday polarimetry allowed us to monitor the processes of electron seeding and acceleration in the second stage.

\footnotetext{
a) Electronic mail: michaeljs_liu@siom.ac.cn.

b) Electronic mail: ruxinli@mail.shcnc.ac.cn

c) Electronic mail: zzxu@mail.shcnc.ac.cn
} 
Electron-positron pair production from vacuum in ultrastrong laser fields

\author{
Baisong Xie* and Ziliang Li \\ College of Nuclear Science and Technology, \\ Beijing Normal University, Beijing 100875, China \\ Email: bsxiedbnu.edu.cn
}

Electron-positron pair production from vacuum in ultrastrong laser fields are reviewed and commented. In particular the approaches of kinetic methods are introduced which include the quantum Vlasov equation (QVE) and Dirac-Heisenberg-Wigner (DHW) formalism. They are used to study the momentum signatures in nonperturbative multiphoton pair production for the general elliptic-polarized fields. It is found that the polarizations will change the momentum spectra and the number density of created particles. Moreover the polarization of external fields could not only change the node structures or even make the nodes disappear but also change the thresholds of pair production. The momentum signatures associated to the node positions in which the even-number photon pair creation process is forbidden could be used to distinguish the orbital angular momentum of created pairs on the momentum spectra. These distinguishable momentum signatures could be relevant for providing the output information of created particles and also the input information of ultrashort laser pulses.

[1] W. Heisenberg and H. Euler, Z. Phys. 98, 714 (1936); J. Schwinger, Phys. Rev. 82, 664 (1951).

[2] Z. L. Li, D. Lu and B. S. Xie, Phys. Rev. D 92, 085001 (2015).

[3] Z. L. Li, D. Lu, and B. S. Xie, Phys. Rev. D 89, 067701 (2014); Z. L. Li, D. Lu, B. S. Xie,

L. B. Fu, J. Liu, and B. F. Shen, Phys. Rev. D 89, 093011 (2014). 
Hartmut Ruhl

Ludwig-Maximilians-University Munich

Theresienstrasse 37, 80333 Munich, Germany

\section{Variations on Mean Field Theory in Strong Optical Fields: A New Simulation Model for Electron-Positron Plasma and Radiation}

With the advent of ultra-strong optical radiation it might be possible to design exotic sources of electron-positron pairs and $\gamma$-radiation as well as novel photonuclear applications.

A prerequiste for any source design for electron-positron pairs and associated radiation, in particular $\gamma$-radiation, in the context of strong optical fields are theoretical models that can be solved efficiently on a computer with a proper understanding of their limitations or degree of universality.

The focus of the presentation is on the derivation and structure of an effective mean field theory for electrons, positrons and radiation derived from the field equations of quantum electrodynamics and a novel method of its numerical implementation.

Unlike most frameworks available in the field at present the mean field approach presented contains all near field interactions of radiating charged particles and anti-particles, can be extended to include spin, spontaneous vacuum breakup and dispersive nonlinear vacuum optics [1].

The numerical implementation of the theoretical framework relies on molecular dynamical equations of motion for radiating nonclassical quasi-particles. The method is essentially grid free and still scales linearly with the number of its quasi-elements. Hence, the numerical implementation of the framework is efficient and can be utilized to predict dispersive and dissipative properties of the quantum vacuum in great detail in the near future.

\section{References}

[1] P. Böhl, B. King, and H. Ruhl. Vacuum high-harmonic generation in the shock regime. Phys, Rev. A, 92:032115, 2015. URL http://dx.doi.org/ 10.1103/PhysRevA.92.032115. 


\title{
High resolution study of transmission resonances for sub-barrier fission at ELI-NP
}

\author{
Deepika Choudhury ${ }^{1}$, A. Krasznahorkay ${ }^{2}$, L. Csige ${ }^{2}$, D. L. Balabanski ${ }^{1}$, S. Coban ${ }^{1,3}$, \\ P. Constantin ${ }^{1}$, M. Csatlos ${ }^{2}$, J. Gulyas ${ }^{2}$ \\ ${ }^{1}$ Extreme Light Infrastructure - Nuclear Physics (ELI-NP), Horia Hulubei National Institute for \\ R\&D in Physics and Nuclear Engineering (IFIN-HH), Bucharest-Magurele, Romania \\ deepika.choudhury@eli-np.ro,dimiter.balabanski@eli-np.ro,paul.constantin@eli-np.ro \\ ${ }^{2}$ Institute of Nuclear Research, Hungarian Academy of Sciences, 4026 Debrecen, Hungary \\ kraszna@atomki.mta.hu,csige.lorant@atomki.mta.hu,csatlos.margit@atomki.mta.hu, \\ gulyas.janos@atomki.mta.hu \\ ${ }^{3}$ Akdeniz University, Dumlupinar Bulvari, 07058 Antalya, Turkey \\ sevdaselek@gmail.com
}

The high intensity ( $10^{4}$ photons/s/eV), high resolution (band width $\geq 0.3 \%$ ) and highly polarized (>99\%) brilliant gamma-beam system (GBS) to be hosted by ELI-NP [1,2], will allow precise photo-nuclear measurements in the $0.2-20 \mathrm{MeV}$ energy range.

One of the experimental studies, which is under preparation at the GBS, aims at high resolution study of transmission resonances as a function of energy, for light actinide nuclei. The study includes mass, atomic number, angular and kinetic energy distribution of fission fragments following the decay of the states in the different minima of the potential energy surface (PES) [3]. It addresses dynamic and clusterization effects in super- and hyper-deformed states. Studies of rare fission events, such as ternary fission and highly asymmetric fission, are also part of the program.

For these studies, we are developing a detector array, called ELI-BIC, which includes a set of four double-sided Frisch-grid Bragg spectrometers. Each spectrometer will be coupled with eight $\Delta \mathrm{E}$-E detectors for the study of ternary fission events.

GEANT4 simulations [4] providing expected beam profile at the target position, estimates the fission fragment emission rates, their mass and charge distribution, fragment paths and ionization in the gas chamber, will be presented for ${ }^{238} \mathrm{U}$ and ${ }^{232} \mathrm{Th}$ target materials.

The ELI-BIC array is being built in collaboration with MTA-ATOMKI, Debrecen. The present status of development of the set-ups and results from test experiments, demonstrating the performance of the detectors, will be presented.

1. N.V. Zamfir, Nucl. "Extreme Light Infrastructure - Nuclear Physics (ELI-NP)", Phys. News 25:3, 34 (2015).

2. O. Adriani et al., “Technical Design Report; EuroGammaS proposal for the ELI-", arXiv:1407.3669v1 [physics.acc-ph]

3. D. L. Balabanski et al., "Photofission experiments at ELI-NP”, Rom. Rep. Phys. 68, S621 (2016)

4. P. Constantin et al., "Simulation of photofission experiments at ELI-NP facility", Nucl. Inst. Meth. 372, 78, 2016 


\title{
Photofission Experiments at the ELI-NP Facility
}

\author{
P. Constantin, D. L. Balabanski, P.V. Cuong, D. Choudhury, L. T. Anh \\ ELI-NP, IFIN-HH, Str. Reactorului 30, 077125 Bucharest-Magurele, Romania \\ paul.constantin@eli-np.ro,dimiter.balabanski@eli-np.ro,phan.cuong@eli-np.ro, \\ deepika.choudhury@eli-np.ro, anh.le@eli-np.ro
}

An extensive experimental program for the study of photofission will take place at the ELINP facility, where different actinide targets will be exposed to a brilliant gamma beam to produce fission fragments. The unique properties of the ELI-NP gamma beam [1], e.g. a spectral density above $10^{4} /(\mathrm{s} \cdot \mathrm{eV})$ and an energy resolution below $0.5 \%$ at an energy up to $19 \mathrm{MeV}$, will offer the possibility to address several of the major open issues in our current understanding of nuclear physics.

Among the many goals of this program [2], we emphasize: the investigation of the fission potential barrier in super- and hyper-deformed actinides with an array of Bragg Ionization Chambers and Thick GEM detectors; the production of neutron-rich exotic isotopes in a radioactive ion beam with an IGISOL-type facility; and the measurement of nuclear g-factors of short-lived isomeric states with an array of HPGe clover detectors.

We will present the status of the development of the above experimental setups and of the test experiments finished and planned for their characterization. Also, benchmark simulations of signal and background rates and of various functional parameters will be shown $[3,4]$.

1. H.R. Weller et al., "Gamma Beam Delivery and Diagnostics", Rom. Rep. Phys. 68, S447S481 (2016).

2. D.L. Balabanski et al., "Photofission Experiments at ELI-NP", Rom. Rep. Phys. 68, S621-S698 (2016).

3. P. Constantin et al., "Simulation of photofission experiments at the ELI-NP facility", Nucl. Inst. Meth. B 372, 78-85 (2016).

4. P. Constantin et al., "Design of the Gas Cell for the IGISOL Facility at ELI-NP", Nucl. Inst. Meth. B (2016), submitted. 


\title{
The C/O Ratio in Stellar Helium Burning: Measurements of the ${ }^{16} \mathrm{O}(\gamma, \alpha){ }^{12} \mathrm{C}$ Reaction With an Optical TPC at HI $\gamma \mathrm{S}^{*}$
}

\author{
M. Gai ${ }^{1}$, M.W. Ahmed ${ }^{2,3}$, I. Gheorghe ${ }^{4,5}$, M. Freer ${ }^{6}$, C.R. Howell ${ }^{2}$, \\ D.P. Kendellen ${ }^{1,2}$, S.R. Stern ${ }^{1}$, V. Zamfir ${ }^{4}$
}

1 LNS at Avery Point, University of Connecticut, Groton, CT 06340-6097, USA

2 TUNL, Duke University, P.O. Box 90308, Durham, NC 27708-0308, USA

3 Dept of Mathematics and Physics, North Carolina Central University, Durham, NC 27707

4 ELI-NP/ IFIN-HH, 30 Reactorului Street, 077125 Magurele, Romania

5 Faculty of Physics, University of Bucharest, RO-077125 Bucharest, Romania

6 School of Physics and Astronomy, University of Birmingham, Birmingham B15 2TT, UK

Gamma-ray beams provide a unique opportunity to address key questions in nuclear astrophysics including the $\mathrm{C} / \mathrm{O}$ ratio in stellar helium burning [the ${ }^{12} \mathrm{C}(\alpha, \gamma){ }^{16} \mathrm{O}$ reaction] that has been recognized as the central problem in nuclear astrophysics. In these studies with gamma-beams one employs the inverse photo-dissociation reaction; e.g the $(\gamma, \mathrm{a})$ or the $(\gamma, \mathrm{p})$ reactions, with a gain in cross section due to detailed balance. The use of a TPC detector as a target, operating with isotopic enriched gas such as the ${ }^{13} \mathrm{CO}_{2}$, leads to a reduced background. We continue our research effort to measure the ${ }^{16} \mathrm{O}(\gamma, \alpha){ }^{12} \mathrm{C}$ reaction at low energies, in an attempt to resolve some of the ambiguities that were recently reviewed [1]. We are developing an isotopic enriched gas system and we formed a new international collaboration that will allow for several future independent analyses of angular distributions of the ${ }^{16} \mathrm{O}(\gamma, \alpha){ }^{12} \mathrm{C}$ reaction [2]. These measurements of very detailed and complete angular distributions at $\mathrm{E}_{\gamma}=9.4$ and $9.5 \mathrm{MeV}$ at $\mathrm{HI} \gamma \mathrm{S}$ with an O-TPC detector operating with $\mathrm{N}_{2} \mathrm{O}$ gas [2], may indeed lead to a resolution of the recently reviewed [1] conflict of the measured E1-E2 mixing phase angles $\left(\phi_{12}\right)$ with unitarity, but further analyses are required to confirm this assertion.

* This work is supported in part by the U.S. Department of Energy, Grants No. DE-FG0294ER40870 and No. DE-FG02-97ER41033, by the NSF: US-Romania, Catalyzing New International Collaboration (NSF-CNIC) award number 1443249, and by the Extreme Light Infrastructure Nuclear Physics (ELI-NP) Phase II, a project co-financed by the Romanian Government and the European Union through the European Regional Development Fund (1/07.07.2016).

[1] AMBIGUITIES OF THE RATE OF OXYGEN FORMATION DURING STELLAR HELIUM BURNING IN THE ${ }^{12} \mathrm{C}(\alpha, \gamma)$ REACTION.

Moshe Gai, Phys. Rev. C 88, 062801(R) (2013).

[2] PRECISION ANGULAR DISTRIBUTION DATA FOR THE ${ }^{16} \mathrm{O}(\gamma, \alpha){ }^{12} \mathrm{C}$ REACTION IN THE REGION OF THE $1^{-}$RESONANCE AT 9.6 MeV.

W.R. Zimmerman, M.W. Ahmed, A. Kafkarkou, I. Mazumdar, J.M. Mueller, L.S. Myers, M.H. Sikora, S. Stave, H.R. Weller, M. Gai, A.G. Swindell.

Bull. Amer. Phys. Soc. 59,\#5(2014)66. 


\title{
Multipole response in atomic nuclei within the Second Random Phase Approximation
}

\author{
Danilo Gambacurta ${ }^{1}$ \\ ${ }^{1}$ ELI-NP, Horia Hulubei National Institute for R\&D in Physics and Nuclear Engineering, \\ 077125 Magurele, Romania \\ danilo.gambacurta@eli-np.ro
}

The isovector electric dipole strength of atomic nuclei is generally fuly exhausted by the isovector giant dipole resonance (IVGDR), located in the energy region between 10-20 MeV. However, in neutron-rich systems, the low-energy electric dipole response is characterized by the appearance of some strength, exhausting a small, tough considerable (typically few percents) fraction of the total E1 strength. For this reason, this mode is usually refereed as Pygmy Dipole Resonance (PDR). It is usually described as the motion of the outermost neutrons (e.g. neutron skin) against a proton-neutron saturated core. The experimental investigation of the IVGDR and PDR is one of the main pillar of the ELI-NP facility, where high-power lasers together with a very brilliant gamma beam will be the main research tools.

From a theoretical point of view, one of the most successful model employed in the description of the nuclear multipole response is the Random Phase Approximation (RPA). In RPA, nuclear modes are described as linear superpositions of 1 particle -1 hole (1p-1h) excitations build on top of a correlated ground state. RPA provides a rather good description of the main properties of the nuclear response, such as the centroid energy and the total stength. However properties like the spreading width and the fragmentation of the strength, or the appearance of anharmonicites cannot be described within the RPA, being it a mean-field based approach. The Second Random Phase Approximation (SRPA) is a natural extension of RPA where more general excitation operators are introduced, containing also 2 particle-2 hole ( $2 \mathrm{p}-2 \mathrm{~h}$ ) excitations. The spreading width can be described because the coupling with the $2 \mathrm{p}-2 \mathrm{~h}$ configurations is fully taken into account. However, due to the numerical effort required, only in the last years, large-scale SRPA calculations have been performed, showing merits and limits of this approach [17].

In this talk, we present an overview of recent applications of the SRPA with the Skyrme and Gogny interactions. Giant resonances in light nuclei will be studied and their properties discussed by using different approximated SRPA schemes [3-4]. After that, the low-energy dipole excitations are analyzed for the stable isotopes $40 \mathrm{Ca}$ and $48 \mathrm{Ca}$ [5]. The presence of a neutron skin in the nucleus $48 \mathrm{Ca}$ would suggest the interpretation of the low-lying response in terms of a PDR like mode. However, RPA calculations are not able to provide any strength in the energy region from 5 to $10 \mathrm{MeV}$. The inclusion and the coupling of $2 \mathrm{p}-2 \mathrm{~h}$ configurations in the SRPA lead to a far better agreement with the experimental data. The composition of the excitation modes (content of $1 \mathrm{p}-1 \mathrm{~h}$ and $2 \mathrm{p}-2 \mathrm{~h}$ configurations), their transition densities and their collectivity (number and coherence of the different contributions) will be analyzed and discussed. After that, some more recent results [6,7], obtained by using a subtraction procedure to overcome double-counting in the SRPA, will be discussed. We will show that this procedure leads to results that are weakly cutoff dependent and that a strong reduction of the SRPA downwards shift with respect to the RPA spectra is found.

1. P. Papakonstantinou and R. Roth,”Second random phase approximation and renormalized realistic interactions”,Phys. Lett. B, 671, 356, (2009).

2. P. Papakonstantinou and R. Roth, "Large-scale second random-phase approximation calculations with finite-range interactions”, Phys. Rev. C, 81, 024317, (2010). 
3. D. Gambacurta, M. Grasso and F. Catara, "Collective nuclear excitations with Skyrme-Second RPA", Phys. Rev. C, 81, 054312, (2010).

4. D. Gambacurta, M. Grasso and F. Catara, "Residual interaction in second random-phase approximation with density-dependent forces: rearrangement terms“, Journal of Physics G, 38, 035103, (2011).

5. D. Gambacurta, M. Grasso, and F. Catara,'Low-lying dipole response in the stable 40,48Ca nuclei with the second random-phase approximation", Phys. Rev. C, 84, 034301, (2011).

6. D. Gambacurta, M. Grasso and J.Engel, "Subtraction method in the second random-phase approximation: First applications with a Skyrme energy functional", Phys. Rev. C, 92, 034303, (2015).

7. D. Gambacurta and M. Grasso, "Second RPA calculations with the Skyrme and Gogny interactions “, The European Physical Journal A, 52, 2016. 


\title{
Studying low-lying electric dipole strength in atomic nuclei with quasi-monoenergetic gamma-rays
}

\author{
U. Gayer ${ }^{1 *}$, H. Pai ${ }^{2}$, P.C. Ries ${ }^{1}$, M. Zweidinger ${ }^{1}$, T. Beck ${ }^{1}$, M. Bhike ${ }^{3}$, N. Cooper ${ }^{4}$, J. Isaak ${ }^{5,6,7}$, \\ Krishichayan $^{3}$, B. Löher ${ }^{7}$, N. Pietralla ${ }^{1}$, D. Savran ${ }^{7}$, M. Scheck ${ }^{8,9}$, M. Schilling ${ }^{1}$, W. Tornow ${ }^{3}$, \\ H. Weller ${ }^{3}$, V. Werner ${ }^{1}$ \\ ${ }^{1}$ IKP TU Darmstadt, Darmstadt, HE, Germany \\ *ugayer@ikp.tu-darmstadt.de \\ ${ }^{2}$ Saha Institute for Nuclear Physics, Kolkata, IN-WB, India \\ ${ }^{3}$ Duke University, Durham, NC, US \\ ${ }^{4}$ University of Richmond, Richmond, VA, USA \\ ${ }^{5}$ ExtreMe Matter Institute, GSI, Darmstadt, HE, Germany \\ ${ }^{6}$ Frankfurt Institute for Advanced Studies, Frankfurt, HE, Germany \\ ${ }^{7}$ GSI Helmholtzzentrum für Schwerionenforschung, Darmstadt, HE, Germany \\ ${ }^{8}$ School of Engineering, University of the West of Scotland, Paisley, GB-RFW, UK \\ ${ }^{9}$ Scottish Universities Physics Alliance, Glasgow, GB-GLG, UK
}

The dominant electric dipole (E1) excitation mode of atomic nuclei is the giant dipole resonance (GDR), which was discovered almost 70 years ago by Baldwin and Klaiber [1]. In a collective model, this excitation mode is understood as an oscillation of the proton body against the neutron body of a nucleus [2]. Systematic experimental investigations suggest that, across the entire nuclear landscape, the GDR cross section is a Lorentzian shape with a width of several $\mathrm{MeV}$ and a centroid energy $\mathrm{E}_{\mathrm{c}}$ with a mass dependence of [3]

$$
\mathrm{E}_{\mathrm{c}}=31.2 \mathrm{MeV} \cdot \mathrm{A}^{-1 / 3}+20.6 \mathrm{MeV} \cdot \mathrm{A}^{-1 / 6}
$$

This places the largest part of the E1 strength above the neutron separation threshold $\mathrm{S}_{\mathrm{n}}$ in most nuclei. Accordingly, $(\gamma, \mathrm{nx})$ reactions have been a standard method for investigations of the GDR cross section.

Especially in the last 20 years, experimental interest has seen a shift to E1 excitation strength close to and below $S_{n}$, where a second collective E1 excitation mode, the pygmy dipole resonance (PDR), is under discussion [4]. It is often understood as an oscillation of the excess neutrons in a neutron-rich nucleus against an $\mathrm{N}=\mathrm{Z}$ core. Although this mechanism is probably not the only one to create pygmy dipole strength (see, e.g. [5]), it is generally agreed that the PDR is structurally different from the GDR.

Being associated with the neutron excess, the PDR should be sensitive to the neutron skin thickness of a nucleus. Therefore, a theory that describes its structure will also put important constraints on the properties of neutron matter in neutron stars [6,7]. Furthermore, nuclei with a large neutron excess are in the path of the astrophysical r-process that is mainly responsible for the synthesis of elements heavier than iron in the universe. The PDR is expected to have a considerable influence on reaction rates in this region of the nuclear chart [8].

A well-suited experimental method to investigate E1 strength below $S_{n}$ is nuclear resonance fluorescence (NRF, see Ref. [9] and references therein). Important advantages of the method are the selectivity of photons to dipole excitations, the model independence of the extracted quantities and the high energy resolution achievable in gamma-ray detection. The advent of 
sources for quasi-monoenergetic polarized gamma-ray sources has considerably increased the sensitivity of NRF experiments to excitation cross sections, branching ratios, angular momentum and parity [10] quantum numbers.

In this talk, recent results of combined NRF experiments with continuous bremsstrahlung at the Superconducting DArmstadt LINear ACcelerator (S-DALINAC [11,12]) and quasimonoenergetic polarized photons at the High-Intensity $\gamma$-ray Source (HIyS [13]) on nuclei around the $\mathrm{A}=50$ and $\mathrm{A}=90$ mass region will be presented.

For an interpretation of the data, known GDR cross sections above $S_{n}$ have been extrapolated to lower energies by means of the statistical model. This way, it is possible to see whether the low-lying E1 strength exhibits properties expected for the low-energy tail of the GDR or if there is additional PDR strength.

This work was supported by research grants SFB 634 and SFB 1245 of the Deutsche Forschungsgemeinschaft.

1. G.C. Baldwin and G.S. Klaiber, "Photo-Fission in Heavy Elements”, Phys. Rev. 71 (1947)

2. M. Goldhaber and E. Teller, “On Nuclear Dipole Vibrations”, Phys. Rev. 74 (1948)

3. J. Speth and A. van der Woude, “Giant resonances in nuclei”, Rep. Prog. Phys. 44 (1981)

4. D. Savran, T. Aumann, A. Zilges, "Experimental studies of the Pygmy Dipole Resonance”, Prog. Part. Nucl. Phys. 70 (2013)

5. V. Derya et al., "Isospin properties of electric dipole excitations in ${ }^{48} \mathrm{Ca}$ ", Phys. Lett. B 730 (2014)

6. B.A. Brown, "Neutron Radii in Nuclei and the Neutron Equation of State”, Phys. Rev. Lett. 85 (2000)

7. B.A. Brown and A. Schwenk, "Constraints on Skyrme equations of state from properties of doubly magic nuclei and ab initio calculations of low-density neutron matter”, Phys. Rev. C 89 (2014)

8. E. Litvinova et al., "Low-lying dipole reponse in the relativistic quasiparticle time blocking approximation and its influence on neutron capture cross sections”, Nucl. Phys. A 823 (2009)

9. U. Kneissl, N. Pietralla, A. Zilges, "Low-lying dipole modes in vibrational nuclei studied by photon scattering”, J. Phys. G: Nucl. Part. Phys. 32 (2006)

10. N. Pietralla et al., "Parity measurements of Nuclear Levels Using a Free-ElectronLaser Generated $\gamma$-Ray Beam”, Phys. Rev. Lett. 88 (2001)

11. A. Richter, “Operational Experience at the S-DALINAC”, Proceedings of the $5^{\text {th }}$ EPAC, IOP Publishing, Bristol (1996)

12. F. Hug et al., "Operational Findings and Upgrade Plans on the Superconducting Electron Accelerator S-DALINAC”, Proceedings of the 2011 PAC, PAC'11 OC / IEEE (2011)

13. H.R. Weller et al., "Research Opportunities at the Upgraded HIyS Facility", Prog. Part. Nucl. Phys. 62 (2009) 


\title{
Monte Carlo simulations for ELI-GANT detection array at ELI-NP
}

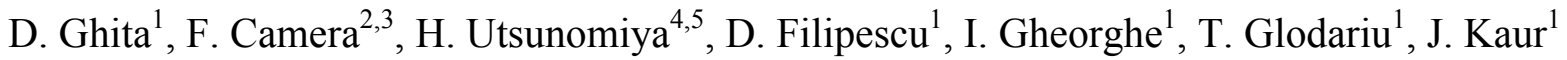 \\ ${ }^{1}$ Extreme Light Infrastructure - Nuclear Physics (ELI-NP) / Horia Hulubei National Institute for \\ R\&D in Physics and Nuclear Engineering (IFIN-HH), Bucharest-Magurele, RO-077125, \\ Romania,dan.ghita@eli-np.ro,dan.filipescu@eli-np.ro,ioana.gheorghe@eli-np.ro, \\ tudor.glodariu@eli-np.ro,jasmeet.kaur@eli-np.ro \\ ${ }^{2}$ University of Milano, Department of Physics, Via Celoria 16, Milano 1-20133, Italy, \\ franco.camera@mi.infn.it \\ ${ }^{3}$ INFN section of Milano Via Celoria 16, Milano 20133, Italy \\ ${ }^{4}$ Department of Physics, Konan University, Okamoto 8-9-1, Kobe 659-8501, Japan, \\ hiro@center.konan-u.ac.jp \\ ${ }^{5}$ Center for Nuclear Study, University of Tokyo, 2-1 Hirosawa, Wako, Saitama 351-0198, Japan
}

The Extreme Light Infrastructure - Nuclear Physics (ELI-NP) is a facility dedicated to nuclear physics research with extreme electromagnetic fields. It will host a system of two high power $10 \mathrm{PW}$ lasers and a very brilliant gamma-beam system. The expected gamma-ray beams with energies up to $20 \mathrm{MeV}, 0.5 \%$ relative energy resolution and $\sim 10^{8}$ photons per second intensity will be employed for precise photonuclear measurements. The experimental program for nuclear studies at ELI-NP involves nuclear resonance fluorescence, gamma above neutron threshold, absolute photon induced reaction cross and photofission experiments, as described at lenght in [1].

Here we report on the status of the experimental setups dedicated to studies of atomic nuclei above the neutron emission threshold using the high energy resolution and high intensity ELI-NP gamma-ray beams. We present the feasibility studies performed using extensive Geant4 simulations, results of detector tests, the status of the data acquisition system, details on the implementation of the mechanical frames.

Nuclear structure experiments will involve photonexcitations of mainly low-spin collective states and the observation of the radiation emitted in the subsequent decays. Energy and angular differential photoneutron reactions and elastic and inelastic $\left(\gamma, \gamma^{\prime}\right)$ photon scattering are proposed to be recorded using a mixed gamma-neutron detection array comprised of $34 \mathrm{LaBr}_{3}$ and $\mathrm{CeBr}_{3}$ $\gamma$-ray scintillation detectors and 62 BC501 liquid scintillator and ${ }^{6} \mathrm{Li}$ glass neutron detectors. Extensive simulations have been performed adapting the detection system to the experimental conditions provided by the ELI-NP gamma beam system, such as the beam time structure and high incident flux.

We report also on the perspectives for nuclear reaction studies, which will be performed by measuring total and partial photoneutron reaction cross sections. Two detection systems are to be implemented: a $4 \pi$ high efficiency neutron detection system for near threshold investigations and a $4 \pi$ flat efficiency neutron detection system for neutron multiplicity sorting experiments. Both detection systems use ${ }^{3} \mathrm{He}$ neutron counters.

1. D. Filipescu, "Perspectives for photonuclear research at the Extreme Light Infrastructure - Nuclear Physics (ELI-NP) facility” Eur. Phys. J. A, 51, 185 (2015). 


\title{
Development of the ELISSA array: first tests and simulations
}

\author{
G.L. Guardo ${ }^{1}$, A. Anzalone ${ }^{1}$, W. Crucillà ${ }^{1}$, M. La Cognata ${ }^{1}$, D. Lattuada ${ }^{1}$, C. Spitaleri ${ }^{1.2}$, A. \\ Taffara $^{1}$

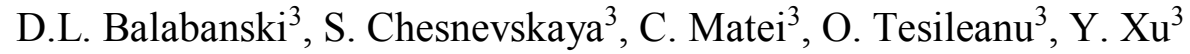 \\ ${ }^{1}$ INFN, Laboratori Nazionali del Sud, Via Santa Sofia 62, 95123 Catania, Italy \\ guardo@lns.infn.it,lacognata@lns.infn.it \\ ${ }^{2}$ Dipartimento di Fisica e Astronomia, Università degli Studi, Via Santa Sofia 64, 95123 \\ Catania, Italy \\ ${ }^{3}$ ELI-NP/IFIN-HH, 30 Reactorului Street, 077125 Magurele, Romania
}

The Extreme Light Infrastructure - Nuclear Physics (ELI-NP) facility, under construction in Magurele near Bucharest in Romania, will provide high-intensity and high-resolution gamma ray beams that can be used to address hotly debated problems in nuclear astrophysics, such as the measurement of controversial ${ }^{12} \mathrm{C}(\alpha, \gamma){ }^{16} \mathrm{O}$ cross section through the ${ }^{16} \mathrm{O}(\gamma, \alpha){ }^{12} \mathrm{C}$ reaction, the accurate measurements of the cross sections of the ${ }^{24} \operatorname{Mg}(\gamma, \alpha){ }^{20} \mathrm{Ne}$ reaction and other photodissociation processes relevant to stellar evolution and nucleosynthesis [1].

For this purpose, a silicon strip detector array (named ELISSA) will be realized in a common effort by ELI-NP and INFN-LNS (Catania, Italy), in order to measure excitation functions and angular distributions over a wide energy and angular range. We performed very accurate GEANT4 simulations in order to optimize resolution, detection efficiency, compactness, granularity, possibility of particle identification and costs. According to our simulations, the final design of ELISSA will be the barrel configuration. This results in a very compact design as the distance target - detector is about $10 \mathrm{~cm}$, leaving open the possibility in the future to pair a neutron detector with the ELISSA. Because of the compact design of the detector, time-of-flight or standard $\Delta \mathrm{E}-\mathrm{E}$ approach cannot be used for particle ID. However, kinematical identification still making possible to separate the reaction of interest from others thanks to the good expected angular and energy resolutions.

A prototype of ELISSA was built and tested at Laboratori Nazionali del Sud (INFN-LNS) in Catania with the support of ELI-NP. In this occasion, we have carried out experiments with alpha sources and with a $11 \mathrm{MeV}{ }^{7} \mathrm{Li}$ beam. We used X3 and QQQ3 silicon-strip position sensitive detectors manufactured by Micron Semiconductor 1td. Thanks to our approach, the first results of those tests show up a very good energy resolution (better than 1\%) and very good position resolution, of the order of $1 \mathrm{~mm}$. At very low energies, below $1 \mathrm{MeV}$, a worse position resolution is found, of the order of $5 \mathrm{~mm}$, but still good enough for the measurement of angular distribution and the kinematical identification of the reactions induced on the target by gamma beams. Moreover, a threshold of $150 \mathrm{keV}$ can be easily achieved with no cooling. We will discuss technical details of the detector and present results regarding Monte Carlo simulation, energy resolution and detection thresholds of ELISSA, the physical cases to be investigated.

Finally, we propose to measure the ${ }^{7} \mathrm{Li}\left(\gamma,{ }^{3} \mathrm{H}\right){ }^{4} \mathrm{He}$ reaction at gamma energies below $11 \mathrm{MeV}$. The reaction is of interest for the longstanding "Cosmological Li problem" and for verifying several recent theoretical predictions. Although most measurements over the last 30 years have concentrated in an energy range below $1.5 \mathrm{MeV}$, measurements at higher energies could restrict the extrapolation to astrophysically important energies. An exploratory experiment to measure the 
${ }^{7} \mathrm{Li}\left(\gamma,{ }^{3} \mathrm{H}\right){ }^{4} \mathrm{He}$ reaction has been approved at High Intensity Gamma Source (HIGS) and preparations are under way to carry out the experiment in 2016-2017.

Extensive theoretical calculations and experimental simulations for the photonuclear reactions involving ${ }^{7} \mathrm{Li}$ and $\mathrm{p}$-process nuclei have been performed as the first feasibility study for the measurements at ELI-NP.

To sum up, these tests, simulations and first experiments allow us to say that the X3 detectors, as well the standard QQQ3 detectors, are perfectly suited for nuclear astrophysics studies with ELISSA.

1. D. Filipescu et al., Eur. Phys. J., A51, 185 (2015). 


\title{
Using nuclear transitions to control and store $x-$ ray photons
}

\author{
Xiangiin Kong ${ }^{1}$, Adriana Pálffy ${ }^{1}$ \\ ${ }^{I}$ Max Planck Institut for Nuclear Physics, Heidelberg, Germany \\ kong@mpi-hd.mpg.de,palffy@mpi-hd.mpg.de
}

Recent years have witnessed the commissioning of coherent X-ray sources opening the new field of x-ray quantum optics [1]. While not yet as advanced as its optical counterpart, the latter may enable coherent control of x-rays, with potential applications for the fields of metrology, material science, quantum information, biology and chemistry. The desirable properties of x-rays are deeper penetration, better focus, no longer limited by an inconvenient diffraction limit as for optical photons, correspondingly spatial resolution, robustness, and the large momentum transfer they may produce. A peculiar circumstance is that x-rays are resonant to either inner shell electron transitions in (highly) charged ions, or transitions in atomic nuclei.

Here, we investigate how to use nuclear transitions in the x-ray regime to manipulate single $\mathrm{x}$-ray quanta. The key for such control is the use of Mössbauer transitions in solid-state targets which enable collective effects to come into play in the nuclear excitation and decay processes. Particularly successful systems to exploit collective effects of nuclei in x-ray single-photon superradiance have proved to be thin-film planar x-ray cavities with an embedded ${ }^{57} \mathrm{Fe}$ nuclear layer, see example in Fig. 1. For instance, recently the groups of J. Evers and R. Röhlsberger could experimentally show that in such cavities it is possible to slow down a narrow-band x-ray pulse due to the resonant interaction with the nuclear layer [2].

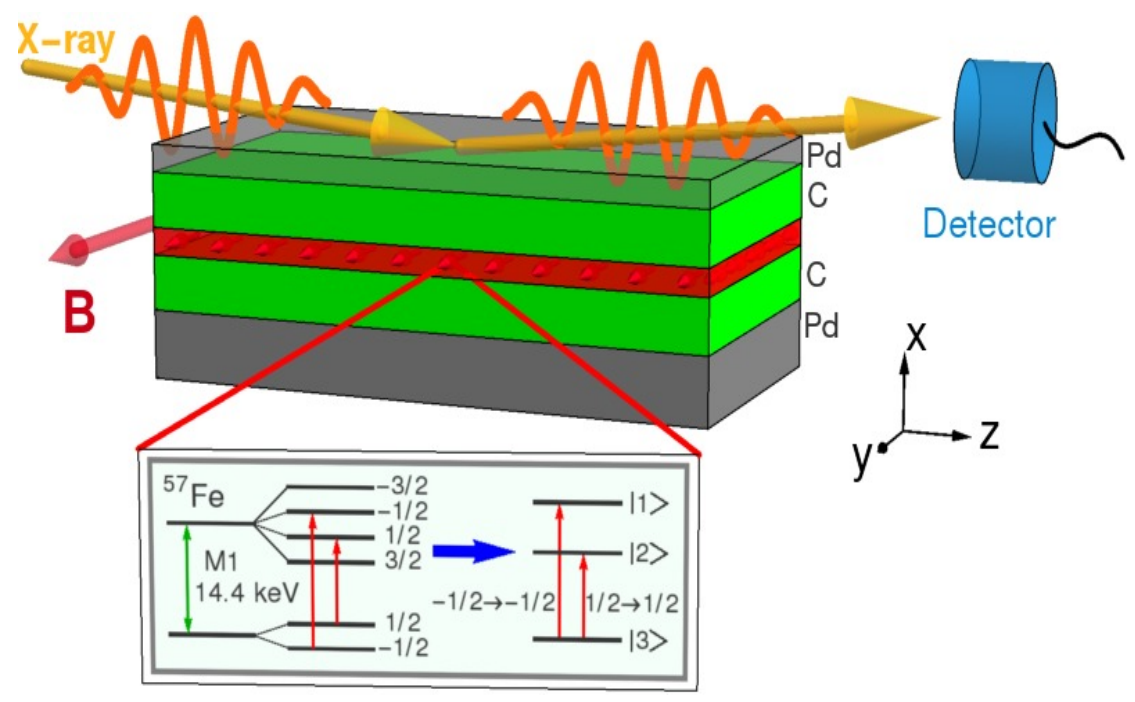

Fig. 1. Thin-film planar cavity setup with $\mathrm{x}$-ray grazing incidence. The cavity consists of a sandwich of Pd and $\mathrm{C}$ layers with a $1 \mathrm{~nm}$ layer containing ${ }^{57} \mathrm{Fe}$ placed at the antinode of the cavity. The nuclei experience a hyperfine magnetic field B (red horizontal arrow). Inset panel: ${ }^{57} \mathrm{Fe}$ level scheme with hyperfine splitting.

In this work we present a theoretical control mechanism for stopping x-ray pulses in resonant nuclear media. We show that narrow-band x-ray pulses can be mapped and stored as nuclear coherence in a thin-film planar x-ray cavity with an embedded iron layer as illustrated in Fig. 1. The pulse is nearly resonant to the $14.4 \mathrm{keV}$ Mössbauer transition in the ${ }^{57} \mathrm{Fe}$ nuclei. The 
role of the control field that is required to manipulate the x-ray pulse is played here by a hyperfine magnetic field which induces interference effects reminding of electromagnetically induced transparency. We show that by switching off the control magnetic field, a narrow-band $\mathrm{x}$-ray pulse can be completely stored in the cavity for approximately hundred ns [3]. Additional manipulation of the external magnetic field can lead to both group velocity and phase control of the pulse in the x-ray cavity sample.

1. B. W. Adams et al., J. Mod. Opt. 60, 2 (2013).

2. K. Heeg et al., Phys. Rev. Lett. 114, 203601 (2015).

3. X. Kong and A. Pálffy, Phys. Rev. Lett. 116, 197402 (2016). 


\title{
Overview of the Gamma Beam Monitoring Instruments at ELI-NP
}

\author{
C. Matei, V. Iancu, A. Pappalardo, G. Suliman, C.A. Ur \\ ELI-NP/IFIN-HH, 30 Reactorului Street, 077125 Magurele, Romania \\ catalin.matei@eli-np.ro
}

The high brilliance Gamma Beam System (GBS) at ELI-NP will deliver quasimonochromatic gamma-ray beams (bandwidth $<0.5 \%)$ with a high spectral density $\left(>10^{4}\right.$ photons $/ \mathrm{s} / \mathrm{eV}$ ) and high degree of linear polarization $(>99 \%)$. The GBS will be delivered in two phases with two separate beam lines: a low-energy gamma-ray line with gamma energies up to 3.5 MeV and a high-energy gamma line with energies up to $19.5 \mathrm{MeV}$.

Optimization and monitoring of the gamma beam with these characteristics is challenging and requires the proper means for accurately measuring the spatial, spectral and temporal characteristics of the gamma-ray beams.

The gamma beam energy spread will be monitored using a large volume HPGe detector with a $\mathrm{NaI}(\mathrm{Tl})$ anti-Compton shield placed in the attenuated beam. Energies above $10 \mathrm{MeV}$ will be monitored using a large volume LaBr3 instead of the HPGe detector [1].

An intensity and polarization monitor is proposed based on the $d(\gamma, n)$ reaction which could be placed in either the low-energy or the high-energy experimental areas. This technique has been tested successfully [2] at the HI $\gamma \mathrm{S}$ facility to measure the beam intensity and polarization.

A thin scintillator sheet with high conversion efficiency would be placed in the gamma beam and imaged with a CCD camera to give insights into the spatial position of the beam and allow quick experimental instrument alignment.

Several additional instruments using Compton scattering and photo-fission are envisioned for measuring the time structure, intensity, and polarization of the beam. Details on the status of these devices will be presented.

1. H.R. Weller et al., "Gamma Beam Delivery and Diagnostics at ELI-NP”, Rom. Rep. in Phys., 68, S447 (2016).

2. C. Matei et al., "Investigation of the $\mathrm{d}(\gamma, \mathrm{n}) \mathrm{p}$ reaction for gamma beam monitoring at ELINP ", Journal of Instrumentation, 11, P05025 (2016). 


\title{
Near threshold photonuclear reactions
}

\author{
I.N.Tsymbalov ${ }^{1,2}$, A.L.Polonski ${ }^{1}$, V.G.Nedorezov ${ }^{1}$, A.B.Savel'ev ${ }^{2}$ \\ ${ }^{1}$ Institute for Nuclear Research RAS, Moscow, Russia \\ ${ }^{2}$ Lomonosov State University, Moscow, Russia \\ vladimir@cpc.inr.ac.ru \\ ivankrupenin2@gmail.com
}

The paper is devoted to the feasibility study of photonuclear reactions at photon energies below $10 \mathrm{MeV}$. This includes the measurement of the total cross sections of photoabsorption, yields and cross sections of photoneutron reactions. New fundamental data on photonuclear reactions (new modes of dipole excitations - pygmy-resonance, neutron widths, polarization effects, etc.) are discussed. Preliminary comparative results obtained with the electron linear accelerator LUE-8 MeV at INR and terawatt femtosecond laser complex of Moscow State University are presented. Mutual use of two different systems provides high accuracy measurements and the development of new technologies. On the basis of numerical simulation and targeted experiments new methods for the study of photonuclear reactions are under development. 


\title{
K Isomers in neutron-rich Rare-earth nuclei*
}

\author{
C.R. Praharaj ${ }^{1}$, Z. Naik ${ }^{2}$, and B.B. Sahu ${ }^{3}$ \\ 1Institute of Physics, Bhubaneswar 751005. India \\ 2Department of Physics, Sambalpur University, Burla 768019. India \\ 3Department of Physics, KIIT Univer, Bhubaneswar 751024. India
}

Isomers are long lived states of nuclei and are quite distinct from other nearby nuclear states. $\mathrm{K}$ isomers in nuclei have considerably larger $\mathrm{K}$ quantum numbers than the usual nuclear states. The electromagnetic transitions to nearby states are severely retarded because of this difference in $\mathrm{K}$ quantum number. Their lifetimes can range from nanoseconds to years. Thus the $\mathrm{K}$ isomers can be useful for energy storage.

We have done theoretical studies $[1,2]$ of the structure of such isomers in nuclei in the rare-earth region and have estimated their lifetimes. These will be reported at the Conference.

${ }^{*}$ CRP acknowledges support of Department of Science and Technology (Project SB/S2/HEP-06/2013).

1. C.R. Praharaj et al., Journal of Physics Conference Series 312, 092052 (2011) .

2. S.K. Ghorui et al., AIP Conference Proceedings 1609, 135 (2014); S.K. Ghorui and C.R. Praharaj, Pramana J. Phys. 82, 659 (2014). 


\title{
High energy coherent X-ray microscopy for mesoscopic materials
}

\author{
Irina Snigireva ${ }^{1}$ and Anatoly Snigirev ${ }^{2}$ \\ ${ }^{1}$ European Synchrotron Radiation Facility (ESRF), Grenoble, France \\ irina@esrf.fr \\ ${ }^{2}$ Baltic Federal University, Kaliningrad, Russia \\ anatoly.snigirev@gmail.com
}

We present a high energy coherent X-ray microscope to study the wide range of natural and artificial mesoscopic materials that are structured on scales of the order of a few to a few hundred nanometers. The concept of the proposed microscope is based on the use of compound refractive lenses allowing to retrieve high resolution diffraction pattern and real-space images in the same experimental setup [1-4]. The microscope operates under a coherent illumination where a diffraction pattern of the specimen is formed in the back focal plane of the condenser and an inverted two-dimensional image of the object is formed by objective lens in the image plane [5]. A high spatial coherence is needed in the imaging mode to ensure a reasonable contrast. The coherence in terms of the angular source size determines the lens angular resolution $(<1 \mu \mathrm{rad})$ to get high resolution diffraction patterns.

Functioning at energies $10-60 \mathrm{keV}$, the microscope is one of the brunches of the multimodal instrument which is under the development at the ID06 ESRF beamline. It consists of the condenser, the objective lens and two X-ray CCD cameras - large area detector for diffraction and high resolution $\mathrm{CCD}$ for imaging. Condenser and objective assemblies are comprised of Beryllium parabolic refractive lenses. Switching from the diffraction mode to the imaging is achieved by placing the objective lens into the beam, and selecting the proper detector. The tunable objective lens offers full-field imaging with variable resolution and field of view. At present, at the maximum magnification a resolution of $100 \mathrm{~nm}$ is achieved.

The microscope was applied for study of natural and synthetic opals, metal inverted photonic crystals and colloidal suspensions $[5,6]$. The combination of the direct-space imaging and high resolution diffraction provide a wealth of information on their local structure and the long range periodic order. The concept of the hard X-ray microscope emerged concomitantly with the realization that the ESRF source upgrade, through the greatly enhanced brilliance and fraction of coherent light, open entirely new frontiers in materials imaging. Short acquisition times with modern area detectors allow to extend the microscope to time-resolved studies of the crystallization dynamics, response of the mesoscopic structures to external stimuli such as mechanical strain, temperature jump or temperature gradient as well as external fields.

1. A. Snigirev, V. Kohn, I. Snigireva, B. Lengeler, Nature, 384 (1996) 49.

2. V. Kohn, I. Snigireva, A. Snigirev, Opt. Comm., 216 (2003) 247.

3. M. Drakopoulos, A. Snigirev, I. Snigireva, J. Schilling, Appl. Phys. Lett., 86 (2005) 014102.

4. P. Ershov, S. Kuznetsov, I. Snigireva, V. Yunkin, A. Goikmann, A. Snigirev, Appl. Cryst. 46 (2013) 1475.

5. A. Bosak, I. Snigireva, K. Napolskii, A. Snigirev, Adv. Mater., 22 (2010) 3256.

6. D. V. Byelov, J.-M. Meijer, I. Snigireva, A. Snigirev, L. Rossi, Esther van den Pol, A. Kuijk, A. Philipse, A. Imhof, A. van Blaaderen, G. Jan Vroege, A. V. Petukhov, RSC Advances, 3 (2013) 15670. 


\title{
The ThomX X-ray source
}

\author{
K. Dupraz ${ }^{1}$, on behalf of the ThomX collaboration \\ ${ }^{1}$ CNRS / LAL, Orsay, France \\ dupraz@lal.in2p3.fr
}

ThomX is a compact Compton machine [1] under construction at LAL (Orsay, France). It is a collaboration between CELIA, ESRF, LAL, LAMS, Néel, and SOLEIL laboratories. It aims at producing quasi-monochromatic X-rays in the energy range 30-90 $\mathrm{keV}$ by use of the Compton back-scattering between electrons bunches and a laser beam. The expected X-ray flux will range between $10^{11}$ and $10^{13}$ photons/s within an energy bandwidth between $1 \%$ and $10 \%$.

To reach this goal, an electron ring of $18 \mathrm{~m}$ circumference with variable energy around $50 \mathrm{MeV}$ and $1 \mathrm{nC}$ current has been designed. Electron bunches will be injected at $50 \mathrm{~Hz}$ by an S-band linear accelerating structure including a photocathode RF gun. Xrays will be produced by a high average power of about $50 \mathrm{~W}$ laser system producing picosecond pulses at $33.3 \mathrm{MHz}$. This laser beam will be enhanced by used of a resonant cavity that bring the laser average power at the interaction point above $300 \mathrm{~kW}$. An X-ray transfer line dedicated to the peculiarity of the Compton angular distribution has been designed to deliver beams with the required characteristics for the different applications situated in an experimental hutch.

Strong technological challenges remain to be resolved for both electron ring and laser system during the commissioning. For the former, a turbulent regime of the electron beam is expected requiring effort on beam dynamics and longitudinal/transverse feedbacks. For the latter, since no industrial system is able to produce such a high average power, a very high finesse optical resonator has to be provided. Running a high finesse cavity filled with a high average power pulsed laser beam in the accelerator environment is an issue.

This presentation will start by a global description of the ThomX machine key elements and by giving the installation status. Then the main technological issues of this machine described above will also be underlined. Finally the foreseen applications exploiting the performance of ThomX will be described $[2,3]$.

1. C. Bruni et al., "ThomX: Technical design report", IN2P3, 00971281 (2014).

2. P. Walter et al., "A new high quality X-ray source for Cultural Heritage", $C$. $R$. Physique, 10, 676 (2009).

3. M. Jacquet and P. Suortti, "Radiation therapy at compact Compton sources", Phys. Med., 31, 596-600 (2015). 


\title{
Exploring Strong-Field QED with Ultra-Intense Lasers
}

\author{
C. D. Murphy ${ }^{1,2}$, C. D. Baird ${ }^{1}$, T. Blackburn ${ }^{3}$, A. Ilderton ${ }^{3}$, J. G. Kirk ${ }^{4}$, S. P. D. Mangles ${ }^{5}$, M. \\ Marklund $^{3}$, P. McKenna ${ }^{6}$, D. Stutman ${ }^{7}$, A. G. R. Thomas ${ }^{8}$ I. C. E. Turcu ${ }^{2,7}$ and C. P. Ridgers ${ }^{1}$ \\ 1- University of York, Heslington, York, YO10 5DD, UK \\ 2- STFC-Rutherford Appleton Laboratory, Harwell Campus, Didcot, Oxfordshire OX11 0QX, UK \\ 3- Department of Applied Physics, Chalmers University of Technology, SE-412 96 Gothenberg, Sweden \\ 4- Max Planck Institute for Nuclear Physics, PO Box 1039 80, 69029, Heidelberg, Germany \\ 5- Imperial College London, South Kensington, London, SW7 2AZ, UK \\ 6- Department of Physics, SUPA, University of Strathclyde, Glasgow G62 6DL, UK \\ 7- National Institute for Physics and Nuclear Engineering, ELI-NP, Bucharest-Magurele, Romania \\ 8- University of Lancaster, Bailrigg, Lancaster LA1 $4 Y W, U K$
}

Current high-power laser facilities can focus light to extremely high intensities $\left(10^{21}-10^{22} \mathrm{Wcm}^{-2}\right)$. At such extreme intensities we are on the verge of a regime where the electromagnetic fields in the laser focus will be so strong that very nonlinear quantum electrodynamics (QED) effects, not yet seen in the laboratory, will play a critical role in determining the plasma dynamics [1]. A 'QED plasma' similar to that present in the magnetospheres of pulsars is created [2]. These nonlinear QED processes become important when the electric field in the electron's rest frame $\left(E_{R F}\right)$ approaches the critical field for $Q E D$ (the Schwinger field $E_{S}=1.3 \times 10^{18} \mathrm{Vm}^{-1}$ ) [3]. Here we outline experimental proposals and completed experimental work which aims at observing and measuring these effects.

This new regime will become accessible with the commissioning of laser systems such as ELI-NP. When operational, it will enable the first exploration of several fundamental strong-field QED processes including very non-linear inverse Compton scattering and the resulting radiation reaction; and very nonlinear Breit-Wheeler pair production. Recently, Ilderton \& Torgrimmson showed that a description of radiation reaction within the framework of strong-field QED is only compatible with a subset of classical theories [4]. The first observation of Breit-Wheeler pair production in the very nonlinear regime will be very important as the cross-section for this process underpins pair cascades in extreme astrophysical environments such as pulsar magnetospheres. Models for these processes now form a foundational element of simulation codes for the interaction of 10PW lasers with matter. Benchmarking the QED model used in these codes is essential if theories of laser generated QED-plasmas are to be built up and future laser-plasma experiments moving beyond today's intensity frontier are to be understood.

[1] C.P. Ridgers et al, Phys. Rev. Lett., 108, 165005 (2012)

[2] P. Goldreich, \& W.H. Julian, ApJ., 157, 869 (1969) ; A.N. Timokhin, MNRAS, 408, 2092 (2010)

[3] F. Sauter, Z. Phys. 69, 742 (1931); W. Heisenberg \& H.Euler, Z. Phys. 98, 714 (1936); J. Schwinger, Phys. Rev., 82, 664 (1951)

[4] A. Ilderton \& G. Torgrimsson, Phys. Lett. B, 725, 481 (2013) 
Characterization of Laser-Compton Sources at LLNL

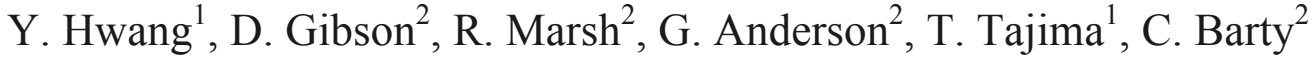

${ }^{1}$ University of California, Irvine, Irvine, CA, USA

${ }^{2}$ Lawrence Livermore National Laboratory, Livermore, CA, USA

Narrow bandwidth (sub-\%), high flux gamma-ray beams enable unique abilities to detect and characterize nuclear materials [1] and can be generated by Compton scattering of laser photons by relativistic electron beam.[2] The bandwidth performance of laser-Compton X-ray and gamma-ray sources is dependent upon high-current, low-emittance accelerator operation and implementation of efficient laser-electron interaction architectures. In order to study these effects, laserCompton $\mathrm{X}$-rays have been produced using the unique compact $\mathrm{X}$-band linear accelerator at LLNL operated in a novel multibunch mode that is scalable to higher photon energies. Results agree very well with modeling predictions.[3,4] Flux, source size and bandwidth of the $30 \mathrm{keV}$ X-ray beam were measured using an X-ray CCD camera and image plates. K-edge absorption images using thin foils confirm the narrow bandwidth (sub-\%) of the laser-Compton source and offer new opportunities for electron beam diagnosis through matched imaging simulation.
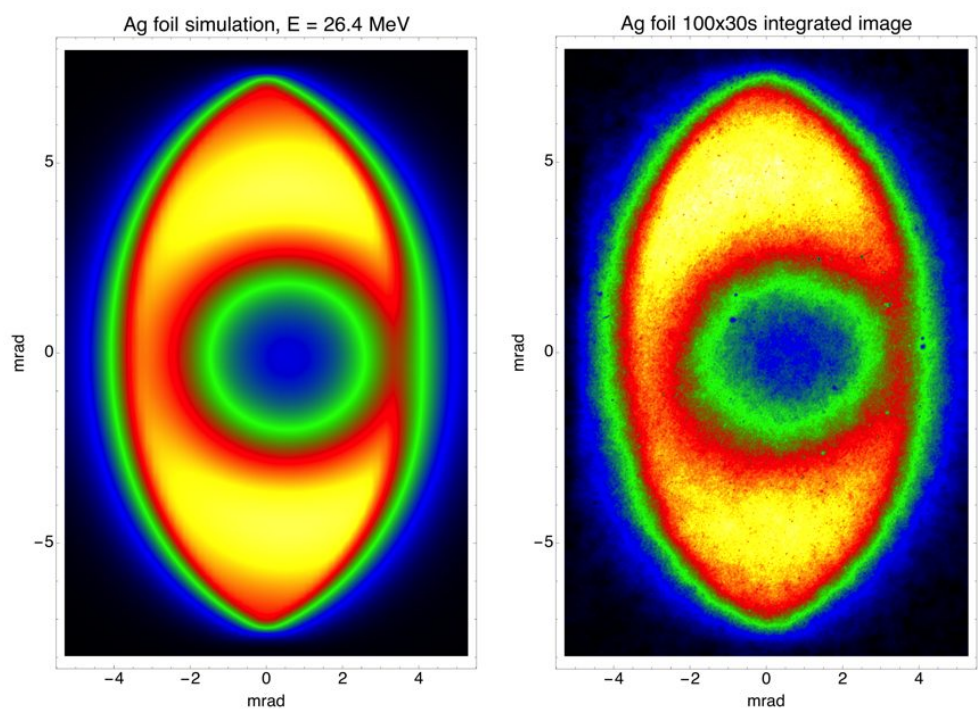

Comparison of data and simulation of $26 \mathrm{keV}$ Laser-Compton X-ray beam image through $50 \mu \mathrm{m}$ Silver foil (K-edge $25.5 \mathrm{keV}$ ), demonstrating the energy-angle correlation and narrow bandwidth of the beam.

This work was performed under the auspices of the U.S. Department of Energy by Lawrence Livermore National Laboratory under Contract DE-AC52-07NA27344. 
1. J. Pruet, D. P. McNabb, C. A. Hagmann, F. V. Hartemann, and C. P. J. Barty, "Detecting clandestine material with nuclear resonance fluorescence," Journal of Applied Physics 99, 123102-123101-123111 (2006)

2. F. V. Hartemann, D. J. Gibson, S. G. Anderson, A. M. Tremaine, P. T. Springer, A. J. Wootton, E. P. Hartouni, and C. P. J. Barty, "High-energy scaling of Compton scattering light sources," Physical Review Special Topics-Accelerators and Beams 8, 100702(100717) (2005)

3. D. J. Gibson, R. A. Marsh, C. P. Barty, and Y. Hwang, "Multi-bunch Operation of a Laser-Compton X-ray Source Based on Compact X-band Accelerator Technology," in High-Brightness Sources and Light-Driven Interactions, OSA technical Digest (online) (Optical Society of America, 2016), paper EM1A.3.

4. Y. Hwang, T. Tajima, G. Anderson, D. J. Gibson, R. A. Marsh and C. P. J. Barty, "LLNL Laser-Compton X-ray Characterization", Proc. $7^{\text {th }}$ Int. Particle Accelerator Conf (IPAC '16), paper TUPOW052, pp. 1885-1888 


\title{
Enhancement of the Superconducting Darmstadt S-DALINAC Accelerator to an Energy- Recovery LINAC
}

\author{
Lars Jürgensen ${ }^{1}$, Michaela Arnold ${ }^{1}$, Thorsten Kürzeder ${ }^{1}$, Norbert Pietralla ${ }^{1}$, Florian Hug ${ }^{2}$ \\ ${ }^{1}$ TU Darmstadt, Darmstadt, Germany \\ ljuergensen@ikp.tu-darmstadt.de,marnold@ikp.tu-darmstadt.de, \\ kuerzeder@ikp.tu-darmstadt.de,pietralla@ikp.tu-darmstadt.de \\ ${ }^{2} I K P$, Mainz, Germany,hug@kph.uni-mainz.de
}

Numerous applications in the field of nuclear photonics are demanding new sources of quasi-mono-energetic gamma-ray beams. Often linear accelerators are most suitable to reach minimal energy spread and high beam current together with high-performance stability and tunability of the beam. Scaling with beam energy and beam current the costs for such a type of machine as well as its energy consumption can easily exceed given budgets. Recent developments in the field of energy recovering technology concerning linear accelerators give promising impulses towards economically efficient machines for the production of ultra-bright photon beams.

The superconducting Darmstadt linear electron accelerator (S-DALINAC) of the Institute for Nuclear Physics at Technische Universität Darmstadt delivers electron beams in cw-mode with energies up to $130 \mathrm{MeV}$. This recirculating accelerator consists of a $10-\mathrm{MeV}$ injector and a $30-\mathrm{MeV}$ main linac where superconducting 3-GHz cavities are operated at a temperature of $2 \mathrm{~K}$ for beam acceleration. During the last shutdown a third recirculation beam line has been installed to further improve the peak energy from approximately $85 \mathrm{MeV}$ to the design value of $130 \mathrm{MeV}$ in cw-operation. Additionally this new scheme offers the opportunity to study energy recovery linac (ERL) mode for a single and a twice recirculating accelerator. The upcoming experimental beam time therefore contains investigation of the ERL mode at the S-DALINAC. We will present the implemented design and give a status report on the project. 
Twisted Gamma-rays from Nonlinear Inverse Compton Scattering of Circular Polarized Light

Masahiro Katoh ${ }^{1 *}$, Yoshitaka Taira ${ }^{2,3}$, Takehito Hayakawa ${ }^{4,5}$

${ }^{1}$ Institute for Molecular Science, National Institutes of Natural Sciences/School of Physical Sciences, SOKENDAI (The Graduate University for Advanced Studies), Myodaiji-cho, Okazaki 444-8585 Japan

2Research Institute for Measurement and Analytical Instrumentation, National Metrology Institute of Japan, National Institute of Advanced Industrial Science and Technology (AIST), Tsukuba Central 2, 1-1-1 Umezono, Tsukuba, Ibaraki 305-8568 Japan

3Department of Physics and Astronomy, Mississippi State University, 355 Lee Blvd., 125 Hilbun Hall, Mississippi State, MS 39762 USA

${ }^{4}$ National Institutes of Quantum and Radiological Science and Technology, Shitakata 2-4, Tokai, Ibaraki 319-1106 Japan

${ }^{5}$ National Astronomical Observatory of Japan, 2-21-1 Osawa, Mitaka, Tokyo 181-8588 Japan

*e-mail:mkatoh@ims.ac.jp

We theoretically show that a photon produced by non-linear Inverse Compton Scattering (ICS) of circularly polarized light is twisted, which means that it possesses a helical wave front and carries orbital angular momentum (OAM) other than spin angular momentum (SAM). Our work explains a recent experimental result on ICS clearly showing an annular intensity distribution as a strong evidence of a vortex beam. We shall discuss a possibility to verify its helical phase structure experimentally. Our work implies that gamma-rays carrying OAM should be produced in various situations in astrophysics in which high-energy electrons and intense circularly polarized light coexist. They may play a critical role in stellar nucleosynthesis with their additional angular momentum. Moreover, non-linear ICS should be the most promising radiation process for realizing a gamma-ray vortex source based on the currently available laser and accelerator technologies, which will be an indispensable tool for exploring gamma-ray vortex science. 


\section{The characterization of $\mathrm{GeV}$ gamma-ray beam via Bremsstrahlung in the storage ring in ELPH, Tohoku University}

Toshiya Muto ${ }^{1}$, Taro Abe ${ }^{1}$, Fujio Hinode ${ }^{1}$, Takatugu Ishikawa ${ }^{1}$, Shigeru Kashiwagi ${ }^{1}$, Hiroki Kanda $^{2}$, Manabu Miyabe ${ }^{1}$, Kenichi Nanbu ${ }^{1}$, Ikuro Nagasawa ${ }^{1}$, Hirotoshi Saito ${ }^{1}$, Yuki Saito ${ }^{1}$, Ken Takahashi $^{1}$ and Hiroyuki Hama ${ }^{1}$

${ }^{1}$ Research center for electron photon science (ELPH), Tohoku University: 1-2-1 Mikamine, Taihaku, Sendai, 982-0826, Japan

muto@lns.tohoku.ac.jp,abe@lns.tohoku.ac.jp,hinode@lns.tohoku.ac.jp, ishikawa@lns.tohoku.ac.jp,kashiwagi@lns.tohoku.ac.jp,miyabe@lns.tohoku.ac.jp, nanbu@lns.tohoku.ac.jp,nagasawa@lns.tohoku.ac.jp,hsaito@lns.tohoku.ac.jp, ysaito@Ins.tohoku.ac.jp,ken_takahashi@lns.tohoku.ac.jp,hama@lns.tohoku.ac.jp ${ }^{2}$ Department of Physics, Tohoku University: 6-3 Aoba, Aramaki, Aoba, Sendai, 980-8578, Japan, kanda@m.tohoku.ac.jp

Booster STorage ring (BST ring) is an electron synchrotron in ELPH, Tohoku University [1][2]. The BST ring has a 4-fold symmetry lattice with quadrupole-sextupole combined magnets for chromatic correction. The BST ring accelerates electron beam from $90 \mathrm{MeV}$ up to $1.31 \mathrm{GeV}$.

There are two gamma ray beamlines at GeV energy order region at the BST ring [3][4]. Inserting an internal target carbon wire with 11 micrometer diameter, the beamlines provide "tagged" photons via Bremsstrahlung for nuclear physics experiments. At the BM5 beamline, tagged photons are with an energy region from $740 \mathrm{MeV}$ to $1150 \mathrm{MeV}$ at ring operation energy of $1200 \mathrm{MeV}$. Total photon tagging rate of BM5 beamline is $\sim 20 \mathrm{MHz}$ in typical repetition mode of the ring in which one cycle is $17 \mathrm{~s}$ and spill time is $10 \mathrm{~s}$. Duty factor is $\sim 60 \%$ in ordinal operation.

By using the beam profile monitor consisted with scintillator strips, the gamma ray profile at the end of BM5 beamline located at $20 \mathrm{~m}$ downstream from the target wire was measured. Horizontal and vertical $\mathrm{rms}$ spatial sizes were derived to be $10.8 \mathrm{~mm}$ and $12.6 \mathrm{~mm}$ respectively. Figure 1 shows the measured beam profile at the experimental area. Using the BST parameters such as beam emittance and Twiss parameters together with Bremsstrahlung property, we have expected the gamma profiles size to be $9.3 \mathrm{~mm}$ and $8.8 \mathrm{~mm}$, respectively.
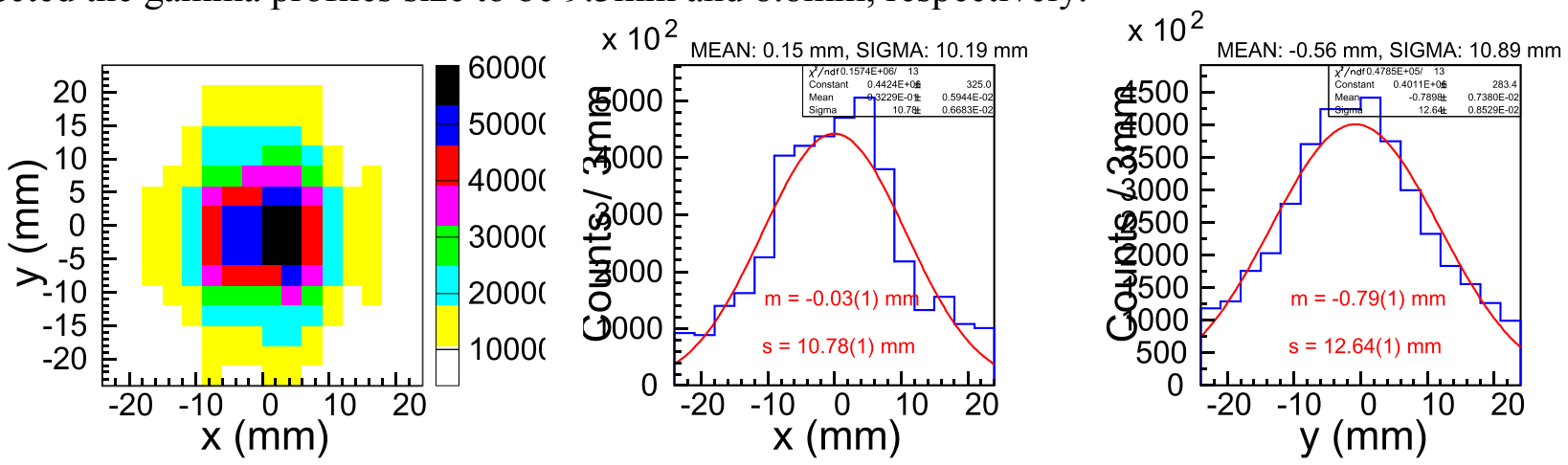

Figure 1 Measured gamma ray beam profile at the experimental area

To resolve this discrepancy, we are taking Coulomb scattering (Mott scattering) on the carbon target into account, which is an elastic scattering between charged particles. By Coulomb scattering, an electron stored in the ring would be disturbed and changed its direction. A portion of Coulomb scattered electrons is able to circulate in the ring. Coulomb scattered electron would hit the carbon wire again and generate gamma ray via Bremsstrahlung. 
It was estimated by the tracking calculation that Coulomb scattered electrons with scattering angle less than $7 \mathrm{mrad}$ was re-stored in the ring. Total cross-section of Coulomb scattering taking electron's survivability into account were calculated to $353 \mathrm{~b}$. This value is pretty large and then $3 \%$ of electrons passing through the carbon wire would be interacting as Coulomb scattering. At least, Coulomb scattered electrons will be survibed for the BST ring damping time of $5.7 \times 10^{-3} \mathrm{~s}$. Total number of Coulomb scattered electron stored in the ring was estimated $1051 N_{e}$, in which $N_{e}$ is number of electrons passes through the carbon wire. The electron horizontal beamsize $\sigma$ at the carbon wire is $0.7 \mathrm{~mm}$ and the carbon wire scans around $4 \sigma$. Therefore, $N_{e}$ is $2.6 \times 10^{-}$ ${ }^{6}$ electrons assuming that primary electron beam profile is normalized Gaussian distribution. On the other hand, to hit the carbon wire, primary electrons must have large betatron amplitude $(>4 \sigma)$. Number of electrons that can pass through the carbon wire in primary beam $N_{\text {large amplitude }}$ is $6.1 \times 10^{-5}$ electrons. Then ratio of Coulomb scattered electrons and primary electron with large betatron amplitude is $\sim 45$. So almost gamma rays via Bremsstrahlung must be generated by Coulomb scattered electrons.

In these considerations, the phase space distribution of electron beam at the carbon wire after Coulomb scattering was calculated by beam tracking in the ring. And by using those electron distributions, gamma ray profiles at $20 \mathrm{~m}$ downstream from the carbon wire was calculated as Fig.2. From Fig.2, the gamma ray beamsizes derived to be $10.6 \mathrm{~mm}$ (horizontal) and $12.7 \mathrm{~mm}$ (vertical) respectively. These are consistent with measured one.
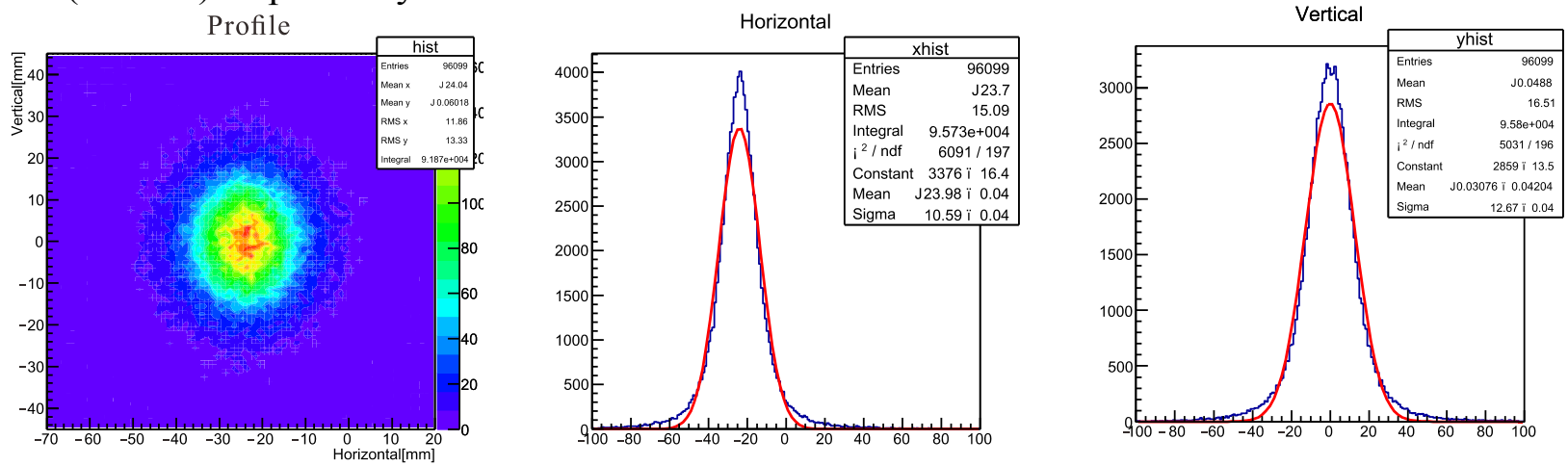

Figure 2 Calculated gamma ray beam profile taking Coulomb scattering into account.

The property of $\mathrm{GeV}$ gamma beamline is characterized by not only BST ring parameters and Bremsstrahlung property, but also Coulomb scattering with the carbon wire. We will report GeV gamma beamline in ELPH and some consideration about its property.

1. F. Hinode et al, "Current Status of a Stretcher-Booster Ring at Tohoku university" Proceedings of the Second Asian Particle Accelerator Conference, (2001) p.106.

2. H. Hama et al, "Current status of a $1.2 \mathrm{GeV}$ Booster electron synchrotoron and implementation for nuclear study at Tohoku university", Proceedings of $18^{\text {th }}$ International Conference on High Energy Accelerators, (2001).

3. H. Yamazaki et al, "The $1.2 \mathrm{GeV}$ photon tagging system at LNS-Tohoku", Nuclear Instruments and Methods in Physics Research A, 536 (2005) p.70.

4. T. Ishikawa et al, "Second GeV tagged photon beamline at ELPH", Nuclear Instruments and Methods in Physics Research A, 622 (2010) p.1. 


\title{
Status of the Development of ELI-NP's Laser Beam Circulator
}

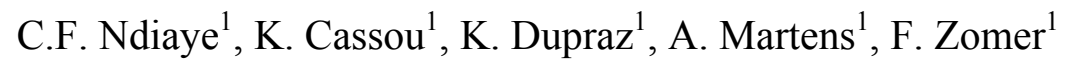 \\ ${ }^{1}$ LAL, Université Paris-Sud, CNRS/IN2P3, Orsay, Bâtiment 200, BP 34, 91898 Orsay cedex, \\ France \\ ndiaye@lal.in2p3.fr,cassou@lal.in2p3.fr,dupraz@lal.in2p3.fr,martens@lal.in2p3.fr, \\ zomer@lal.in2p3.fr
}

In the context of the R\&D program of the EuroGammaS consortium, a collaboration between different academic and industry partners for the Nuclear Physics pillar of the European project Extreme Light Infrastructure (ELI-NP), two multipass Gamma-ray Compton machines are to be built in Măgurele, Romania with photons of tunable energy between $0.2 \mathrm{MeV}$ and up to $20 \mathrm{MeV}$. These machines will have a spectral density an order of magnitude superior to best current machines $\left(\sim 10^{\wedge} 4 \mathrm{~s}^{-1} \mathrm{eV}^{-1}\right.$ at peak energy).

To achieve the required brilliance, the Gamma beam will be produced from the interaction between a relativistic electron bunch and a train of 32 pulses of a high power laser, both at 100 Hz. The spatial and temporal superimposition of the electron and laser beams is challenging and requires state-of-the-art precision machinery and techniques.

Due to the high number of optics (around 120), the surface defects of the mirrors are yet another key aspect to consider in order to obtain a good quality Gamma beam.

A prototype of the multi-pass systems is currently under construction and should be finished by the summer of 2016 .

The challenges, advances and technical choices in the optical design of the Gamma Beam System will be exposed in this work. 


\section{Compton sources for the observation of elastic photon-photon scattering events}

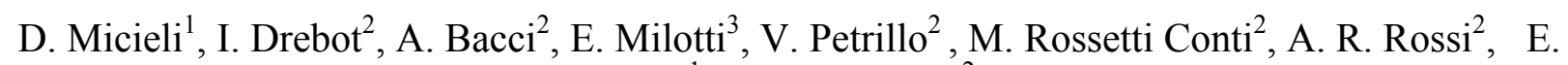
Tassi $^{1}$ and L. Serafini ${ }^{2}$

${ }^{1}$ Università degli Studi della Calabria, Arcavata di Rende (Cosenza), Italy micielidavide@gmail.com; enrico.tassi@fis.unical.it;

${ }^{2}$ INFN-Sezione di Milano,Università degli Studi, via Celoria 16, 20133, Milano, Italy Illya.Drebot@mi.infn.it; Alberto.Bacci@mi.infn.it;Petrillo@mi.infn.it; Marcello.Rossetti@mi.infn.it; Andrea.Rossi@mi.infn.it; Luca.Serafini@mi.infn.it;

${ }^{3}$ Università di Trieste and INFN-Sezione di Trieste, Via Valerio 2, 34127 Trieste, Italy milotti@,ts.infn.it

We present the design of a photon-photon collider based on conventional Compton gamma sources for the observation of elastic gamma-gamma scattering. Two symmetric electron beams, generated by photocathodes and accelerated in linacs, produce two primary gamma rays through Compton back scattering with two high energy lasers. The elastic photon-photon scattering is analyzed by start-to-end simulations from the photocathodes to the detector. A new Monte Carlo code has been developed ad hoc for the counting of the QED events. Realistic numbers of the secondary gamma yield, obtained by using the parameters of existing or approved Compton devices, a discussion of the feasibility of the experiment and of the nature of the background are presented. 


\section{Compton scattered $x$-gamma rays with orbital momentum V.Petrillo ${ }^{1}$, I. Drebot ${ }^{1}$, G. Dattoli ${ }^{2}$, F. Nguyen ${ }^{2}$, L.Serafini \\ ${ }^{1}$ INFN-Sezione di Milano, Università degli Studi, via Celoria 16, 20133, Milano, Italy Petrillo@mi.infn.it; Illya.Drebot@mi.infn.it; Luca.Serafini@mi.infn.it \\ ${ }^{2}$ ENEA, Via E. Fermi, Frascati (Rome), Italy Giuseppe.Dattoli@enea.it; Federico.Nguyen@Enea.it}

We study the possibility of producing x-gamma rays with orbital angular momentum by means of the inverse Compton back-scattering between a high brightness electron beam and a twisted laser pulse. We use the classical electrodynamics retarded fields for evaluating the orbital angular momentum of the radiation and connecting it to that of the primary laser pulse. We then propose the dimensioning of a linearly polarized x-ray source with orbital angular momentum, starting from the parameters of operating Thomson set-ups. a discussion of the feasibility of the experiment and of the nature of the background are presented. 


\title{
Ponderomotive Broadening in Nonlinear Compton Scattering
}

\author{
Sergey G. Rykovanov ${ }^{1}$, Vasily Yu. Kharin ${ }^{1}$, Daniel Seipt ${ }^{2,3}$ \\ ${ }^{1}$ Helmholtz Institute Jena, Froebelstieg 3, 07743 Jena, Germany \\ S.Rykovanov@gsi.de,V.Kharin@gsi.de \\ ${ }^{2}$ Lancaster University, Physics Department, Lancaster, LA1 4YB United Kingdom \\ ${ }^{3}$ The Cockcroft Institute Daresbury Laboratory, Daresbury, WA4 4AD United Kingdom \\ d.seipt@lancaster.ac.uk
}

Inverse Compton Scattering (ICS) is a valuable source of X-and gamma-rays for various applications in medicine, materials science and nuclear physics [1,2]. Main advantage of ICS sources is their ability to provide a very narrow bandwidth $(<1 \%)$ photon spectrum, albeit with a very low conversion efficiency. One way to dramatically increase the total photon yield, is to increase the laser photon density, i.e. increase the laser pulse peak intensity. Unfortunately, scattering laser intensity is strongly limited by the fact that the generated spectrum can be nonlinearly broadened due to the ponderomotive force, and a bandlike structure can appear in the fundamental frequency as well as its harmonics [3-6] even for rather low values of laser pulse amplitude $a_{0}=e A_{L} / m c^{2}$, where $e$ and $m$ are the absolute values of electron charge and mass, $c$ is the speed of light in vacuum and $A_{L}$ is the peak value of the laser pulse vector potential. For example, such moderate laser pulse amplitude as $a_{0}=0.2$ already leads to broadening on the order of $4 \%$.

In this contribution, analytical and numerical results of photon energy-angular spectrum calculation for the case of the nonlinear ICS are presented. Using novel geometrical method of spectrum calculation, it is shown that the interplay between laser pulse chirping and amplitude modulation can lead to interesting spectral features, related to the catastrophe theory. It is also demonstrated, that the nonlinear broadening can be mitigated by appropriately choosing the laser pulse frequency chirp. Results obtained by using purely classical and QED calculations are compared.

1. V.G. Nedorezov, A.A. Turinge, and Y.M. Shatunov, "Photonuclear experiments with Compton-backscattered gamma beams", Physics Uspekhi, 47, 341 (2004)

2. E. Esarey, S. K. Ride, and P. Sprangle, "Nonlinear Thomson scattering of intense laser pulses from beams and plasmas", Physical Review E, 48, 3003 (1993)

3. F.V. Hartemann, A. L. Troha, N. C. Luhmann, and Z. Toffano, "Spectral analysis of the nonlinear relativistic Doppler shift in ultrahigh intensity Compton scattering", Physical Review E, 54, 2956 (1996)

4. D. Seipt, V. Kharin, S. Rykovanov, A. Surzhykov, and S. Fritzsche, "Analytical results for non-linear Compton scattering in short intense laser pulses", Journal of Plasma Physics, 82, 2 (2016)

5. S.G. Rykovanov, C.G.R. Geddes, C.B. Schroeder, E. Esarey, and W.P. Leemans, "Controlling the spectral shape of nonlinear Thomson scattering with proper laser chirping", Physical Review Accelerators and Beams, 19, 030701 (2016)

6. V.Yu. Kharin, D. Seipt, and S.G. Rykovanov, "Temporal laser-pulse-shape effects in nonlinear Thomson scattering", Physical Review A, 93, 063801 (2016) 
Plasma Guiding and Scattering Microstructures for Laser Driven Thompson Sources

Vidmantas Tomkus ${ }^{1}$, Lukas Rimgaila ${ }^{1}$, Valdemar Stankevic ${ }^{1}$, Gediminas Raciukaitis ${ }^{1}$

${ }^{1}$ Center for Physical Sciences and Technology, Savanoriu Ave. 231, LT-02300, Vilnius, Lithuania,vidmantas.tomkus@ftmc.lt,lukas.rimgaila@ftmc.lt, valdemar.stankevic@ftmc.lt, gediminas.raciukaitis@ftmc.lt

All-laser driven Thompson sources produce quasi-monoenergetic tunable X-ray and $\gamma$-photon beams $(10 \mathrm{keV}$ to $>20 \mathrm{MeV})$ with the energy spread $25-50 \%, 10^{7}$ photons per shot and are increasingly used in biomedical imaging, non-destructive testing and low energy nuclear physics [1]. Further increase of brightness and lowering of the bandwidth is related to the higher energy of accelerated electrons, better control of the beam emittance and the increased interaction length with the laser Scattering Beam (SB).

In this report, several configurations of the supersonic micronozzles tuned for the formation of tailored density profile of the guiding of laser Direct Beam (DB) and conical plasma mirrors for the increased interaction length with the laser SB were analyzed. Using ANSYS CFX software the row of 5-7 sapphire micronozzles in the range of the output diameter between 100 $\mu \mathrm{m}$ and $400 \mu \mathrm{m}$ were optimized to ensure the sharp drop of the plasma density for the controlled electron injection into the laser-plasma wakefield accelerator and following gradually increase of the density to compensate the diffraction of the laser DB and lower the electron beam emittance.

The array of the truncated conical micromirrors with the diameter of the cone of $100-200$ $\mu \mathrm{m}$ and hollow aperture with the diameter of $30 \mu \mathrm{m}$ were designed using Lumerical software to form the Bessel Light Pulse (BLP) from an incident Gaussian pulse and manufactured in the fused silica plate. The cone acts as a reflecting axicon with plasma mirror and forms the diffractionless BLP zone along the axis of SB propagation. For the cone apex angle of 170 degrees the length of non-diffracting BLP extends for $0.57-1.14 \mathrm{~mm}$. After the shot the SB is moved to the next cone cell. The conical mirrors with the reflectivity close to $100 \%$ have no dispersion and the group velocity of the BLP can be adjusted by changing the apex angle of the cone. Radially polarized BLP provides with the longitudinal acceleration-deceleration and the transverse focusing-defocusing of the electron beam depending on the BLP phase. The radial panderomotive force and ion channel arising from the interaction with electron beam helps to collimate the electron bunch [2].

The designed micronozzles and conical micromirrors were inscribed inside the transparent plates by modification of the bulk material and formation of the nanogratings due to the nonlinear interaction with the laser beam. The inscribed samples were selectively etched in the diluted hydrofluoric acid to remove the modified material. Different etching rates between the laser-modified and the unmodified material allow the formation of structures with the high length to diameter aspect ratio reaching (5000:1) in sapphire and (200:1) in the fused silica. The etched surface quality was optimized to get the roughness of 100-200 nm. Proposed microstructures are integrated into the laser system consisting of two separately controllable DB and SB pulse compressors, focusing and delay lines to optimize the acceleration of monoenergetic electrons and brightness of generated X-rays.

1. G. Golovin, et all.: "Intrinsic beam emittance of laser accelerated electrons measured by xray spectroscopic imaging”, Sci. Rep., 6, 24622 (2016)

2. M.-W. Lin, et all.: "Direct electron acceleration in plasma waveguides for compact highrepetition-rate x-ray sources," J. Phys. B: At. Mol. Opt. Phys., 47, 234002 (2014) 


\section{Beam dynamics studies for the RF Linac of the ELI-NP Gamma Beam System.}

C. Vaccarezza ${ }^{1}$, D. Alesini ${ }^{1}$, A. Bacci ${ }^{2}$, M. Bellaveglia ${ }^{1}$, G. Campogiani ${ }^{3}$, G. Di Pirro ${ }^{1}$, I. Drebot $^{2}$, A. Gallo ${ }^{1}$, A. Giribono ${ }^{3}$, A. Ghigo ${ }^{1}$, S. Guiducci ${ }^{1}$, A. Mostacci ${ }^{3}$, D. Palmer ${ }^{2}$, L.

Palumbo $^{3}$, V. Petrillo ${ }^{2}$, A. R. Rossi ${ }^{2}$, L. Sabbatini ${ }^{1}$, L. Serafini ${ }^{2}$, A. Vannozzi ${ }^{1}$, A. Variola ${ }^{1}$

IINFN-LNF,Frascati,Italy, Cristina.Vaccarezza@Inf.infn.it,David.Alesini@Inf.infn.it, Marco.Bellaveglia@lnf.infn.it, Giampiero.DiPirro@lnf.infn.it, Alessandro.Gallo@,lnf.infn.it, Andrea.Ghigo@,lnf.infn.it, Susanna.Guiducci@lnf.infn.it, Lucia.Sabbatini@,lnf.infn.it, Alessandro.Vannozzi@lnf.infn.it, Alessandro.Variola@lnf.infn.it

2INFN-MI, Milano, Italy, Alberto.Bacci@mi.infn.it, Illya.Drebot@mi.infn.it, Dennis.Palmert@mi.infn.it, Vittoria.Petrillo@mi.infn.it, Andrea.Rossi@mi.infn.it, Luca.Serafini@mi.infn.it

${ }^{3}$ University of Rome La Sapienza, Italy, Giovanna.Campogiani@uniroma1.it, Anna.Giribono@uniromal.it,Andrea.Mostacci@uniroma1.it, Luigi.Palumbo@uniromal.it

The ELI-NP GBS is a high spectral density and monochromatic $\gamma$ ray source based upon the inverse Compton scattering effect now under construction in Magurele. Its relevant specifications are brilliance higher than $10^{21}, 0.5 \%$ monochromaticity, $5000 \mathrm{ph} / \mathrm{sec} . \mathrm{eV}$ spectral density, and a 0.2-19.5 MeV energy tunability.

Strong requirements are set for the electron beam dynamic: the control of both the transverse normalized emittance $\varepsilon_{n}$ and the energy spread to optimize the spectral density and guarantee the mono chromaticity of the emitted radiation. On this basis the RF Linac optimization has been performed for the designed energy range, a jitter sensitivity analysis of the machine has been performed and the commissioning operation has been addressed. The simulations results hare here presented. 


\title{
Pygmy dipole resonance in atomic nuclei
}

\author{
V.Yu. Ponomarev \\ Technical University of Darmstadt, Darmstadt, Germany \\ ponomare@theorie.ikp.physik.tu-darmstadt.de
}

The pygmy dipole resonance (PDR) is a low-energy debris of the $1 \hbar \omega$ E1-strength which is pushed by an isovector residual interaction to higher energies to form the giant dipole resonance. It exhausts about $1 \%$ of the EWSR below the particle threshold. High energy resolution experiments reveal fine structure of the PDR in many nuclei. The studies of the PDR fine structure performed within the Quasiparticle-Phonon model, will be reported. Excited states are described by a wave function which includes one-, two-, and three-phonon configurations, i.e. the configuration space in calculations below the threshold is almost complete. Some particular features of the PDR excitation in different nuclear reactions will be also discussed. 
Index

\section{A}

Afanasev, Andrei 70

Ahmed, Mohammad 46

Albert, Felicie 124

\section{B}

Barty, Christopher 52

Blackburn, Tom 152

Bohlen, Simon 153

Brenner, Ceri 116, 117

\section{C}

Camera, Franco 24

Campbell, Edward Michael 51

Cassou, Kevin 38

Chen, Jianhui 68

Cherepy, Nerine 138

Choudhury, Deepika 170

Constantin, Paul 171

Covington, Aaron 154

Cwiok, Mikolaj 121, 122

\section{D}

Downer, Michael 106, 107

Downie, Evangeline 45

Dupraz, Kevin 186

\section{E}

Ejiri, Hiro 113

Erickson, Anna 57, 58

Esarey, Eric 97

\section{F}

Favalli, Andrea 126

Filipescu, Dan 177

\section{G}

Gai, Moshe 88, 172

Gambacurta, Danilo 173, 174

Gaul, Erhard 130

Gayer, Udo 175, 176

Geddes, Cameron 93

Gibson, David 39, 40

Gordon, Daniel 31

Guardo, Giovanni Luca 178, 179

Guenther, Marc 78, 79

Gunst, Jonas 44
H

Hajima, Ryoichi 35, 36

Hanayama, Ryohei 148, 149

Hannachi, Fazia 53

Hayakawa, Takehito 143

Howell, Calvin 84

Hwang, Yoonwoo 188, 189

J

Jensen, Erk 66

Jentschel, Michael 80

Jovanovic, Igor 61, 62

Juergensen, Lars Erik 190

K

Katoh, Masahiro 191

Kim, Hyung Taek 95

Kondo, Kiminori 104, 105

Kong, Xiangjin 180, 181

Kosuge, Atsushi 140, 141

L

Ledoux, Robert 56

Lee, Kitae 132

Liang, Edison 32

Litvinenko, Vladimir 37

Liu, Jiansheng 94

Lozovoy, Vadim 155

Ludewigt, Bernhard 125

M

Marklund, Mattias 29, 150

Marsh, Roark 118

Martens, Aurelien 120

Matei, Catalin 182

Meezan, Nathan 50

Miron, Catalin 26

Mishima, Kenji 102

Miyamoto, Shuji 67

Mueller, Jonathan 111

Murokh, Alex 139

Murphy, Chris 187

Muto, Toshiya 192, 193
$\mathbf{N}$

Nakai, Mitsuo 156

Nakamura, Tatsufumi 101

Ndiaye, Cheikh Fall 194

Nedorezov, Vladimir 183

Nees, John 133

Negoita, Florin 134, 135

Niculae, Dana 25

Nishiuchi, Mamiko 54

O

Oberstedt, Andreas 127

Ohgaki, Hideaki 112

\section{$\mathbf{P}$}

Park, Seong Hee 157

Peatross, Justin 158, 159

Petrillo, Vittoria 195, 196

Pietralla, Norbert 42

Plumeri, Stephane 110

Ponomarev, Vladimir 200

Praharaj, C Rajendra 184

Q

Qiao, Bin 161

Qi, Rong 160

$\mathbf{R}$

Rangacharyulu, Chary 69

Roth, Markus 100

Ruhl, Hartmut 169

Rykovanov, Sergey 197 
Sakai, Yusuke 96

Sang, Haibo 162, 163

Savran, Deniz 85

Schumaker, Will 30

Seiboth, Frank 77

Serebryakov, Dmitry 164

Sergeev, Alexander 28

Seya, Michio 59, 60

Shen, Lei 165

Shima, Tatsushi 119

Siders, Craig 136

Snigireva, Irina 185

Snigirev, Anatoly 75, 76

$\mathrm{T}$

Tanaka, Kazuo 22

Tang, Chuanxiang 71

Tantawi, Sami 74

Tomkus, Vidmantas 198

Tonchev, Anton 89

Tsymbalov, Ivan 166

U

Umstadter, Donald 92

Ur, Calin 23

V

Vaccarezza, Cristina 199

Vogel, Sven 63

W

Wagner, Florian 131

Wang, Wentao 167

Watari, Takeshi 103

Werner, Volker 86, 87

Wu, Ying 34

$\mathrm{Wu}$, Yuanbin 151

$\mathbf{X}$

Xie, Baisong 168

$\mathbf{Z}$

Zilges, Andreas 43

Zomer, Fabian 142 


\section{Day at a glance}

\begin{tabular}{|c|c|c|c|c|}
\hline & Sunday, 10/16 & & Monday & $0 / 17$ \\
\hline & & 7:00 & Registra & n \& Breakfast \\
\hline & & $8: 15$ & Openin & Cypress Room \\
\hline & & $8: 30$ & Mo1-1 & Tanaka \\
\hline & & $8: 55$ & Mo1-2 & Ur \\
\hline & & 9:20 & Mo1-3 & Camera \\
\hline & & $9: 45$ & Mo1-4 & Niculae \\
\hline & & $10: 10$ & Mo1-5 & Miron \\
\hline & & $10: 25$ & Break, T & Dolphins \\
\hline & & $10: 45$ & Mo2-1 & Sergeev \\
\hline & & $11: 10$ & Mo2-2 & Marklund \\
\hline & & $11: 25$ & Mo2-3 & Schumaker \\
\hline & & $11: 50$ & Mo2-4 & Gordon \\
\hline & & $12: 15$ & Mo2-5 & Liang \\
\hline & & $12: 40$ & Lunch, ' & e Dolphins \\
\hline & & $14: 00$ & Mo3-1 & $\mathrm{Wu}$ \\
\hline & & $14: 25$ & Mo3-2 & Hajima \\
\hline & & $14: 50$ & Mo3-3 & Litvinenko \\
\hline & & $15: 15$ & Mo3-4 & Cassou \\
\hline & & $15: 40$ & Mo3-5 & Gibson \\
\hline & & $16: 05$ & Break, T & Dolphins \\
\hline $17: 00$ & Registration & $16: 25$ & Mo4-1 & Pietralla \\
\hline & $17: 00$ - 19:00 & $16: 50$ & Mo4-2 & Zilges \\
\hline & & $17: 15$ & Mo4-3 & Gunst \\
\hline 18:00 & Welcome Reception, & $17: 30$ & Mo4-4 & Downie \\
\hline & Plaza & $17: 55$ & Mo4-5 & Ahmed \\
\hline & & $18: 20$ & No-Hos & Jinner \\
\hline 20:00 & & $20: 30$ & Mo5 & Special Session \\
\hline & & & on Futu & Gamma Systems \\
\hline & & $21: 30$ & & \\
\hline
\end{tabular}

\begin{tabular}{|l|l|l|}
\multicolumn{3}{|c|}{ Tuesday, 10/18 } \\
7:00 & Registration \& Breakfast \\
8:30 & Tu1-1 & Meezan \\
\hline 8:55 & Tu1-2 & Campbell \\
9:20 & Tu1-3 & Barty \\
\hline 9:45 & Tu1-4 & Hannachi \\
\hline 10:00 & Tu1-5 & Nishiuchi \\
\hline 10:25 & Break, The Dolphins \\
\hline 10:45 & Tu2-1 & Ledoux \\
\hline 11:10 & Tu2-2 & Erickson \\
\hline 11:35 & Tu2-3 & Seya \\
\hline 12:00 & Tu2-4 & Jovanovic \\
\hline 12:25 & Tu2-5 & Vogel \\
\hline 12:50 & Lunch, The Dolphins \\
\hline 14:00 & Tu3-1 & Jensen \\
\hline 14:25 & Tu3-2 & Miyamoto \\
\hline 14:50 & Tu3-3 & Chen \\
\hline 15:15 & Tu3-4 & Rangacharyulu \\
\hline 15:40 & Tu3-5 & Afanasev \\
\hline 16:05 & Tu3-6 & Tang \\
\hline 16:30 & Break, The Dolphins \\
\hline 16:50 & Tu4-1 & Tantawi \\
\hline 17:15 & Tu4-2 & Snigirev \\
\hline 17:40 & Tu4-3 & Seiboth \\
\hline 18:05 & Tu4-4 & Guenther \\
\hline 18:20 & Tu4-5 & Jentschel \\
\hline 18:45 & Break, The Dolphins \\
\hline 19:00 & Tu5 & Poster Session \& \\
\hline & & Networking, \\
\hline 22:00 & & Point Lobos \\
\hline & & \\
\hline
\end{tabular}

\section{Subject Categories}

ELI-NP Gamma-Ray Facility \& Research Program

Ultrahigh Intensity Lasers \& Nuclear Physics

Accelerator-based Compton Sources

Photo-nuclear Physics

\section{Extreme Lasers and Applications}

Nuclear Security Applications

High Energy Photon Systems \& Applications

Advanced Optics \& Accelerator Technology 


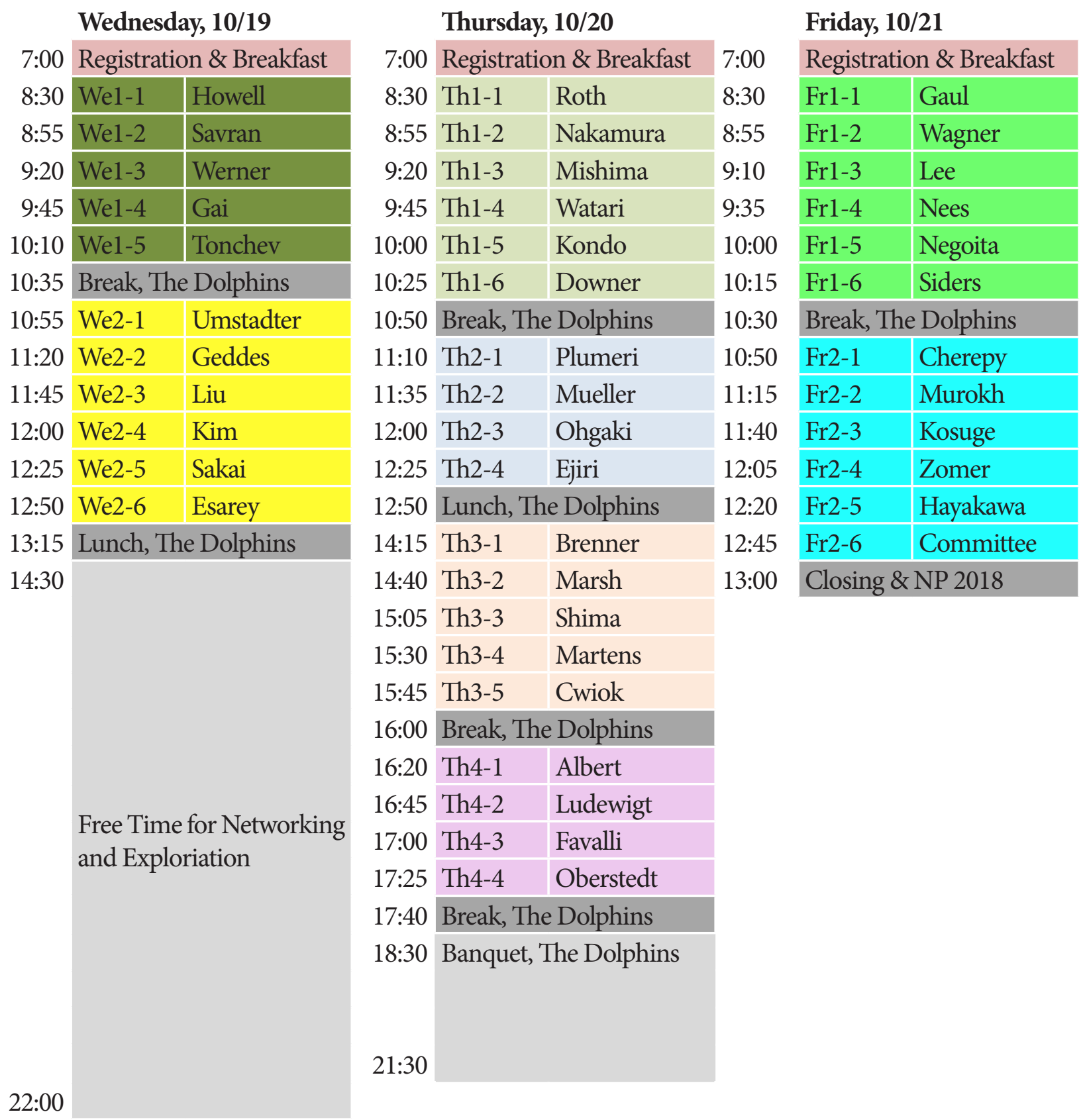

\section{Subject Categories}

Nuclear Structure \& Astrophysics
Compton Sources Based on Intense Lasers
Photon-based Particles Sources
Nuclear Security \& Materials Management
Enabling Technologies for Nuclear Photonics
Isotope-specific Detection, Assay and Imaging
Intense Laser Driven Sources and Technology
Gamma Source Technologies \& Applications




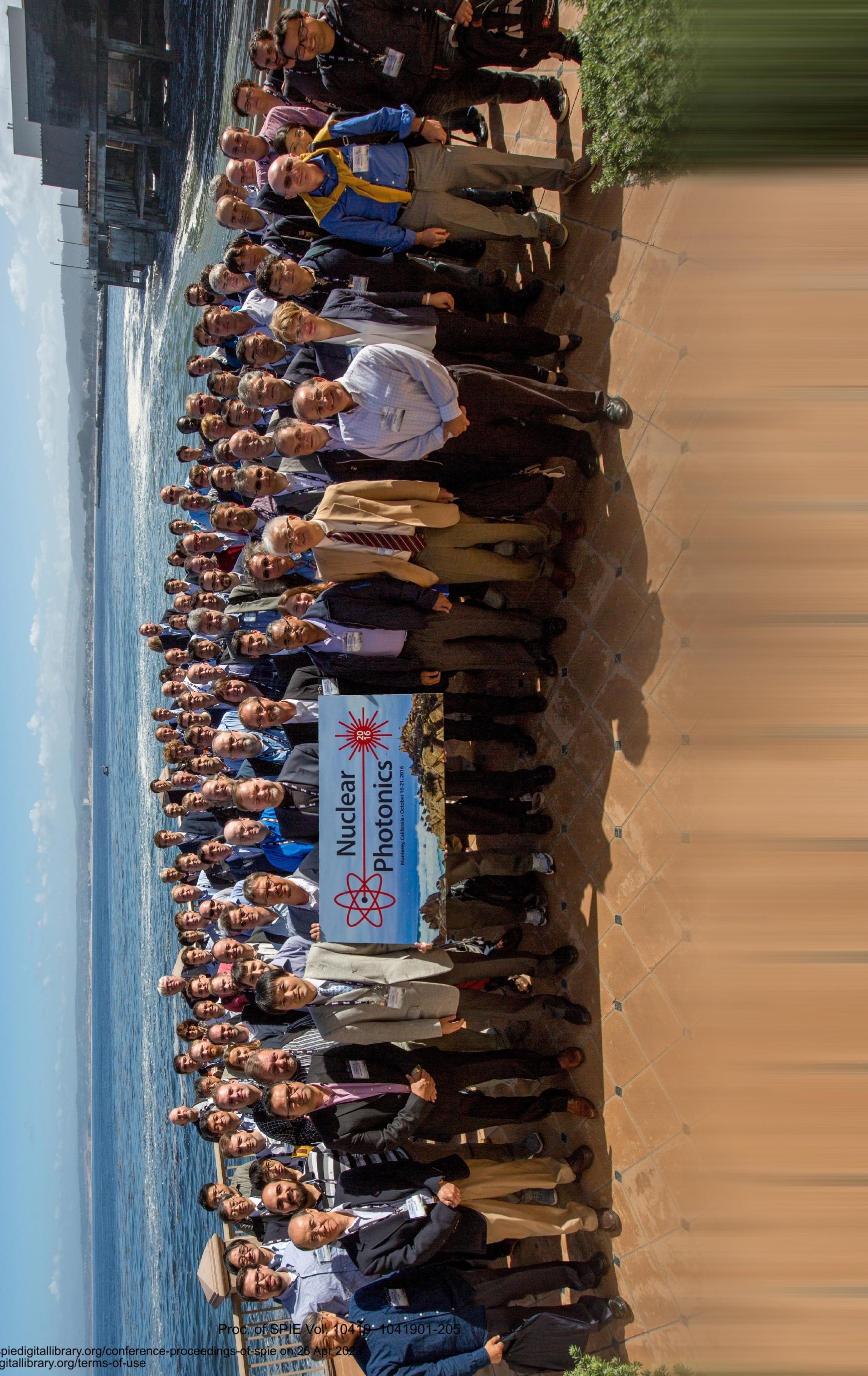



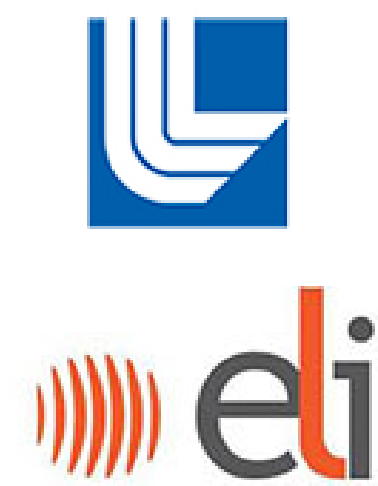

Nuclear Physics
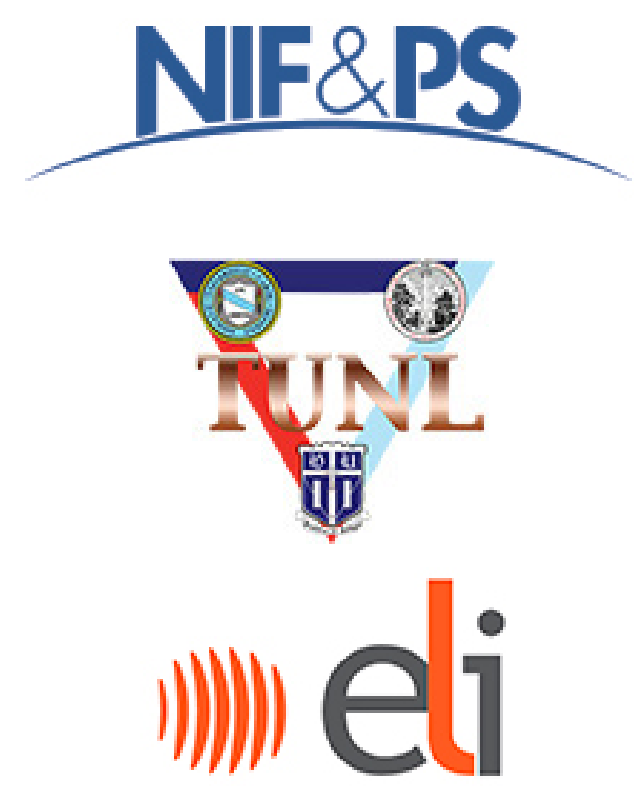

delivery consortium
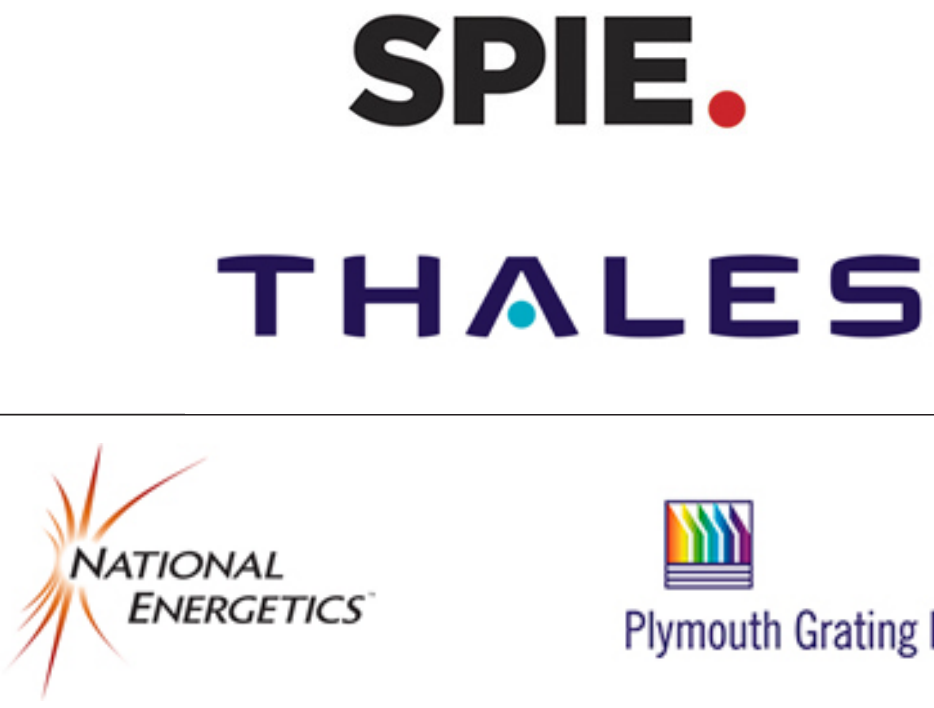

Plymouth Grating Laboratory

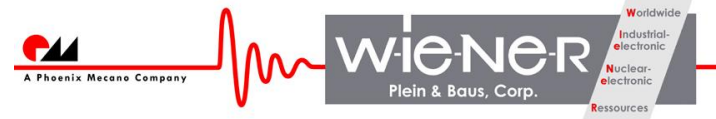

Proc. of SPIE Vol. 10419 1041901-206

Downloaded From: https://www.spiedigitallibrary.org/conference-proceedings-of-spie on 26 Apr 2023 Terms of Use: https://www.spiedigitallibrary.org/terms-of-use 